\title{
Novel aspects of insulin resistance and type 2 diabetes mellitus: iron metabolism, the complement system, and liver cirrhosis
}

Citation for published version (APA):

Wlazlo, N. (2014). Novel aspects of insulin resistance and type 2 diabetes mellitus: iron metabolism, the complement system, and liver cirrhosis. [Doctoral Thesis, Maastricht University]. Maastricht University. https://doi.org/10.26481/dis.20140514nw

Document status and date:

Published: 01/01/2014

DOI:

10.26481/dis.20140514nw

Document Version:

Publisher's PDF, also known as Version of record

Please check the document version of this publication:

- A submitted manuscript is the version of the article upon submission and before peer-review. There can be important differences between the submitted version and the official published version of record.

People interested in the research are advised to contact the author for the final version of the publication, or visit the DOI to the publisher's website.

- The final author version and the galley proof are versions of the publication after peer review.

- The final published version features the final layout of the paper including the volume, issue and page numbers.

Link to publication

\footnotetext{
General rights rights.

- You may freely distribute the URL identifying the publication in the public portal. please follow below link for the End User Agreement:

www.umlib.nl/taverne-license

Take down policy

If you believe that this document breaches copyright please contact us at:

repository@maastrichtuniversity.nl

providing details and we will investigate your claim.
}

Copyright and moral rights for the publications made accessible in the public portal are retained by the authors and/or other copyright owners and it is a condition of accessing publications that users recognise and abide by the legal requirements associated with these

- Users may download and print one copy of any publication from the public portal for the purpose of private study or research.

- You may not further distribute the material or use it for any profit-making activity or commercial gain

If the publication is distributed under the terms of Article $25 f a$ of the Dutch Copyright Act, indicated by the "Taverne" license above, 
Novel aspects of insulin resistance and type 2 diabetes mellitus:

Iron metabolism, the complement system, and liver cirrhosis 
Copyright Nick Wlazlo, Maastricht 2014

No part of this book may be reproduced or transmitted in any form or by any means, without prior permission in writing by the author, or when appropriate, by the publishers of the publications.

Layout: Tiny Wouters

Cover: Jos Wlazlo

Production: GVO drukkers \& vormgevers B.V. | Ponsen \& Looijen, Ede ISBN: 978-90-6464-768-0

Printing of this thesis was financially supported by Boehringer Ingelheim B.V. and Servier Nederland Farma B.V. 


\title{
Novel aspects of insulin resistance and
}

\author{
type 2 diabetes mellitus:
}

\section{Iron metabolism, the complement system, and liver cirrhosis}

\section{PROEFSCHRIFT}

ter verkrijging van de graad van doctor aan de Universiteit Maastricht, op gezag van de Rector Magnificus, Prof. Dr. L.L.G. Soete volgens het besluit van het College van Decanen, in het openbaar te verdedigen op woensdag 14 mei 2014 om 16.00 uur

door

Nick Wlazlo

Geboren te Sittard 


\section{Promotor}

Prof. dr. C.D.A. Stehouwer

\section{Copromotores}

Dr. M.M.J. Van Greevenbroek

Dr. B. Bravenboer

\section{Beoordelingscommissie}

Prof. dr. T. Unger (voorzitter)

Prof. dr. P.C. Dagnelie

Dr. G.H. Koek

Prof. dr. P.A.J. Schrauwen

Prof. dr. C.J. Tack, Radboud UMC 
Age quod Agis 



\section{Contents}

$\begin{array}{lll}\text { Chapter } 1 \quad \text { General introduction } & 9\end{array}$

$\begin{array}{llr}\text { Chapter } 2 & \text { Methodological considerations } & 19\end{array}$

$\begin{array}{lll}\text { Chapter } 2.1 & \text { Study populations } & 21\end{array}$

Chapter 2.2 The use of calibrated data in prospective studies 27

Chapter 2.3 Measures of glucose tolerance, insulin resistance, inflammation, 31 hepatocellular damage, and liver fat

Chapter 2.4 Interpretation of longitudinal data analyses

Chapter 2.5 Mediation analyses in aetiological biomedical research: the influence of random error

\section{Chapter 3 Iron metabolism}

Chapter 3.1 The role of iron metabolism in the metabolic syndrome and type 2 diabetes mellitus

Chapter 3.2 Iron metabolism is associated with adipocyte insulin resistance and plasma adiponectin

Chapter 3.3 Iron metabolism is prospectively associated with insulin resistance and glucose intolerance over a 7-year follow-up period

Chapter 4 The complement system

Chapter 4.1 The complement system in relation to the metabolic syndrome and type 2 diabetes mellitus

Chapter 4.2 Low-grade inflammation and insulin resistance independently explain substantial parts of the association between body fat and serum $\mathrm{C} 3$

Chapter 4.3 Complement factor 3 is associated with insulin resistance and with incident type 2 diabetes mellitus over a 7-year follow-up period 
Chapter 4.4 Activated complement factor 3 is associated with liver fat and liver enzymes

Chapter 4.5 Soluble C5b-C9, but not C5a, is cross-sectionally associated 179 with human fatty liver disease

Chapter 5 Diabetes mellitus and cirrhosis

Chapter 5.1 The relation between diabetes mellitus and liver cirrhosis

Chapter 5.2 High prevalence of diabetes mellitus in patients with liver cirrhosis

Chapter 5.3 Diabetes mellitus at the time of diagnosis of cirrhosis is associated with higher incidence of spontaneous bacterial peritonitis, but not with increased mortality

Chapter 6 Summary and discussion 243

Samenvatting voor niet-ingewijden

Dankwoord

Curriculum vitae

Scientific Output 


\section{Chapter 1}

General introduction 
Chapter 1 


\section{Diabetes mellitus}

Diabetes mellitus is one of the most common metabolic diseases, currently affecting 346 million people worldwide (1). Due to rising obesity rates (2), its prevalence is expected to rise to 552 million in 2030 (3), and in the Netherlands, its prevalence is expected to rise to 1.3 million individuals (8\%) in 2025 (4). Although (well-treated) diabetes may itself be asymptomatic, (long-term) micro- and macrovascular complications of the disease can cause significant morbidity and mortality. Micro-vascular complications include diabetic retinopathy, which accounts for $5 \%$ of cases of blindness worldwide (5), diabetic nephropathy, which is the leading cause of end-stage kidney disease (6), and diabetic neuropathy, which causes painful paraesthesias in one-third of patients with diabetes (7). In addition, patients with diabetes mellitus have a two-fold increased risk for macrovascular diseases such as coronary heart disease and ischaemic stroke (8), and also a two-fold increased risk of (cardiovascular) death compared to subjects without diabetes (9). As such, it is estimated that diabetes mellitus caused 4.6 million deaths worldwide in 2011 (3).

Diabetes mellitus is actually a group of metabolic diseases characterized by elevated plasma glucose levels (i.e. hyperglycaemia), as a result of defects in insulin secretion and/or insulin action (10). The diagnosis is established on elevated fasting glucose ( $>7.0 \mathrm{mmol} / \mathrm{l}$ ) or elevated 2-hour postload glucose $(>11.1 \mathrm{mmol} / \mathrm{l}$ after $75 \mathrm{~g}$ oral glucose tolerance test) on at least 2 occasions, or on a random, non-fasting glucose of $>11.1 \mathrm{mmol} / \mathrm{l}$ in combination with hyperglycaemic symptoms (polyuria, polydipsia, unexplained weight loss) $(10,11)$. Type 1 diabetes mellitus comprises $5-10 \%$ of cases with diabetes, and results from auto-immune destruction of beta cells in the pancreas, leading to absolute insulin deficiency. The majority of individuals with diabetes (90-95\%), however, suffer from type 2 diabetes mellitus (T2DM). The main characteristic of this type of diabetes is that tissues (muscle, adipose tissue, liver) have become less sensitive to the actions of insulin. This increases whole body insulin demand and eventually results in insufficient insulin secretion to overcome this insulin resistance (10). Obesity and physical inactivity are considered the most important risk factors for T2DM $(12,13)$, and are thought to play a major role in the development of insulin resistance early in the pathogenesis of T2DM.

\section{Other features of insulin resistance}

Disorders in glucose metabolism due to insulin resistance are usually not isolated, but accompanied by several other metabolic changes. Initially known as the insulin resistance syndrome', the metabolic syndrome represents this clustering of risk factors for T2DM and cardiovascular diseases, and it believed to be driven to an important extent by obesity, insulin resistance, and microvascular dysfunction $(14,15)$. 
In addition to the presence of (central) obesity and hyperglycaemia, several other metabolic abnormalities often occur as a result of impaired actions of insulin on lipid metabolism and (micro)vascular function (16-19), including decreased plasma high-density lipoprotein (HDL) cholesterol levels, increased plasma triglyceride levels, and hypertension. Together, these metabolic abnormalities are also associated with a two-fold increased risk of cardiovascular diseases and death (20).

Recently, increased hepatic fat accumulation was identified as a feature of the metabolic syndrome (21-23), and the spectrum of this so-called non-alcoholic fatty liver disease (NAFLD) ranges from simple steatosis to steatohepatitis (NASH), and eventually cirrhosis $(24,25)$. NAFLD is present in $40-70 \%$ of individuals with T2DM, $80-95 \%$ of obese individuals, and $65 \%$ of overweight individuals, compared with $25 \%$ of normal weight individuals (26). It is increasingly recognised that the presence of NAFLD, in addition to traditional risk factors, further contributes to the increased risk of cardiovascular events $(27,28)$. In particular, individuals with NASH have a two-fold increased risk of cardiovascular death, and a ten-fold increased risk of liver-related death $(29,30)$. Given the recent increase in the prevalence of NAFLD in the (Western) population, and the fact that $5 \%$ of patients with NAFLD eventually progress to liver cirrhosis (29), the absolute number of patients with NASH cirrhosis is expected to increase dramatically.

\section{Aetiological factors in the development of insulin resistance}

As described above, the development of insulin resistance is an early key element in the pathophysiology of T2DM and the metabolic syndrome. Over the past decades, it has been established that obesity and particularly abdominal and/or visceral fat accumulation are associated with both a local and systemic inflammatory response (31-36). This low-grade inflammation (LGI) in fat and/or liver is, among other things, characterized by elevated secretion and elevated circulating levels of several proinflammatory cytokines, such as tumour necrosis factor- $\alpha$ (TNF- $\alpha$ ) and interleukin- 6 (IL-6) $(31,32,37)$, and non-esterified fatty acids (NEFAs) (38-40). These cytokines and NEFAs are believed to impair metabolic actions of insulin, such as insulin-mediated glucose uptake and insulin-mediated suppression of lipolysis, through both direct and indirect effects on insulin signal transduction (41-44). In agreement with these current views, intravenous administration of TNF- $\alpha$, IL-6, and NEFAs has been shown to cause IR in humans $(45,46)$, and IL-6, C-reactive protein (CRP), and NEFAs have been associated with the development of T2DM in epidemiological studies (47-49).

Although there is growing evidence that (circulating) cytokines and NEFAs are an important causal factor in the development of insulin resistance in several tissues, the mechanisms that trigger the inflammatory response in adipose tissue, and perhaps also the liver, are less clear. Several (intermediate) endogenous and exogenous stress 
signals have been suggested as inducers of the inflammatory response that leads to insulin resistance and T2DM: fatty acids and fatty acid intermediates (36), adipocyte hypoxia (50), gut-derived endotoxins (51), oxidative stress (52), endoplasmatic reticulum stress (53), advanced glycation end products (AGEs) (54), and many more. This thesis focusses on three novel aspects intertwined with inflammation that may also be considered in the aetiology of insulin resistance and T2DM: i.e. 1) iron metabolism (55), 2) the complement system (56), and 3) liver cirrhosis (57). General information on these three matters, as well as a topic-specific introduction into their potential involvement in the pathogenesis of insulin resistance and T2DM, is given in the corresponding chapters hereafter.

\section{Outline and aims of the thesis}

It is well established that lifestyle factors, such as diet, physical inactivity and obesity constitute important determinants of insulin resistance and associated metabolic abnormalities such as hepatic steatosis and dyslipidaemia, which eventually lead to the development of T2DM. Although (obesity-associated) low-grade inflammation is believed to play a key role in the induction of insulin resistance, it remains unclear which processes initiate the inflammatory response, and eventually lead to insulin resistance. The central aim of this thesis was to investigate three novel aetiological factors and/or mechanisms that may be involved in the pathogenesis of insulin resistance and T2DM in humans: iron metabolism, the complement system, and liver cirrhosis.

In this thesis we used observational studies and epidemiological analyses to study aetiological relations. We provide a detailed description of the two study populations used, some key methodological aspects, and the different kinds of statistical methods that are used in this thesis, in Chapter 2. In Chapter 3, we investigated the role of iron metabolism and iron-metabolism-related factors in relation to insulin resistance and glucose intolerance using cross-sectional (Chapter 3.2) and prospective data (Chapter 3.3). In Chapter 4, we examined three aspects of the complement system in relation to obesity, insulin resistance, T2DM, and fatty liver disease. We firstly explored the cross-sectional association between several measures of adiposity and plasma C3 levels (Chapter 4.2), and additionally investigated longitudinal associations between plasma C3 levels and measures of insulin resistance and glucose tolerance (Chapter 4.3). Also, we investigated whether plasma C3a levels, as a marker of proximal complement activation (Chapter 4.4), and plasma levels of $\mathrm{C} 5 \mathrm{a}$ and sC5b-C9, as markers of distal complement activation (Chapter 4.5), were cross-sectionally associated with hepatic steatosis and markers of hepatocellular injury. In Chapter 5, we discussed currently available data in the literature on the bi-directional pathophysiological relationship between T2DM and liver cirrhosis (Chapter 5.1). Moreover, we 
investigated the prevalence of T2DM in patients with liver cirrhosis (Chapter 5.2), as well as its impact on the prognosis of patients with cirrhosis (Chapter 5.3) in a retrospective cohort. Finally, in Chapter 6 the main findings of the studies in this thesis are summarized and discussed, with implications and suggestions for future research. 


\section{References}

1. Danaei G, Finucane MM, Lu Y, Singh GM, Cowan MJ, Paciorek CJ, Lin JK, Farzadfar F, Khang YH, Stevens GA, Rao M, Ali MK, Riley LM, Robinson CA, Ezzati M, Global Burden of Metabolic Risk Factors of Chronic Diseases Collaborating G: National, regional, and global trends in fasting plasma glucose and diabetes prevalence since 1980: systematic analysis of health examination surveys and epidemiological studies with 370 country-years and 2.7 million participants. Lancet 2011;378:31-40

2. Finucane MM, Stevens GA, Cowan MJ, Danaei G, Lin JK, Paciorek CJ, Singh GM, Gutierrez HR, Lu Y, Bahalim AN, Farzadfar F, Riley LM, Ezzati M, Global Burden of Metabolic Risk Factors of Chronic Diseases Collaborating G: National, regional, and global trends in body-mass index since 1980: systematic analysis of health examination surveys and epidemiological studies with 960 country-years and 9.1 million participants. Lancet 2011;377:557-567

3. International Diabetes Federation. Idf diabetes atlas: the global burden, 5th edition [online] [article online], 2011. Accessed September 2013

4. Baan CA, van Baal PH, Jacobs-van der Bruggen MA, Verkley H, Poos MJ, Hoogenveen RT, Schoemaker CG: [Diabetes mellitus in the Netherlands: estimate of the current disease burden and prognosis for 2025]. Ned Tijdschr Geneeskd 2009;153:1052-1058

5. Resnikoff S, Pascolini D, Etya'ale D, Kocur I, Pararajasegaram R, Pokharel GP, Mariotti SP: Global data on visual impairment in the year 2002. Bulletin of the World Health Organization 2004;82:844-851

6. White SL, Chadban SJ, Jan S, Chapman JR, Cass A: How can we achieve global equity in provision of renal replacement therapy? Bulletin of the World Health Organization 2008;86:229-237

7. Abbott CA, Malik RA, van Ross ER, Kulkarni J, Boulton AJ: Prevalence and characteristics of painful diabetic neuropathy in a large community-based diabetic population in the U.K. Diabetes Care 2011; 34:2220-2224

8. Emerging Risk Factors C, Sarwar N, Gao P, Seshasai SR, Gobin R, Kaptoge S, Di Angelantonio E, Ingelsson E, Lawlor DA, Selvin E, Stampfer M, Stehouwer CD, Lewington S, Pennells L, Thompson A, Sattar N, White IR, Ray KK, Danesh J: Diabetes mellitus, fasting blood glucose concentration, and risk of vascular disease: a collaborative meta-analysis of 102 prospective studies. Lancet 2010;375:2215-2222

9. Emerging Risk Factors C, Seshasai SR, Kaptoge S, Thompson A, Di Angelantonio E, Gao P, Sarwar N, Whincup PH, Mukamal KJ, Gillum RF, Holme I, Njolstad I, Fletcher A, Nilsson P, Lewington S, Collins R, Gudnason V, Thompson SG, Sattar N, Selvin E, Hu FB, Danesh J: Diabetes mellitus, fasting glucose, and risk of cause-specific death. N Engl J Med 2011;364:829-841

10. American Diabetes A: Diagnosis and classification of diabetes mellitus. Diabetes Care 2009;32 Suppl 1:S62-67

11. WHO: Definition, diagnosis and classification of diabetes mellitus. Report of a WHO Consultation. Part 1: Diagnosis and classification of diabetes mellitus. . In WHO/NCD/NCS/992 Geneva, Switzerland, 1999

12. Sullivan PW, Morrato EH, Ghushchyan V, Wyatt HR, Hill JO: Obesity, inactivity, and the prevalence of diabetes and diabetes-related cardiovascular comorbidities in the U.S., 2000-2002. Diabetes Care 2005; 28:1599-1603

13. Snijder MB, Dekker JM, Visser M, Bouter LM, Stehouwer CD, Kostense PJ, Yudkin JS, Heine RJ, Nijpels G, Seidell JC: Associations of hip and thigh circumferences independent of waist circumference with the incidence of type 2 diabetes: the Hoorn Study. Am J Clin Nutr 2003;77:1192-1197

14. Alberti KG, Zimmet P, Shaw J: The metabolic syndrome--a new worldwide definition. Lancet 2005;366: 1059-1062

15. Grundy SM, Cleeman JI, Daniels SR, Donato KA, Eckel RH, Franklin BA, Gordon DJ, Krauss RM, Savage PJ, Smith SC, Jr., Spertus JA, Costa F, American Heart A, National Heart L, Blood I: Diagnosis and management of the metabolic syndrome: an American Heart Association/National Heart, Lung, and Blood Institute Scientific Statement. Circulation 2005;112:2735-2752

16. Groop LC, Bonadonna RC, DelPrato S, Ratheiser K, Zyck K, Ferrannini E, DeFronzo RA: Glucose and free fatty acid metabolism in non-insulin-dependent diabetes mellitus. Evidence for multiple sites of insulin resistance. J Clin Invest 1989;84:205-213 
17. Malmstrom R, Packard CJ, Caslake M, Bedford D, Stewart P, Yki-Jarvinen H, Shepherd J, Taskinen MR: Defective regulation of triglyceride metabolism by insulin in the liver in NIDDM. Diabetologia 1997; 40:454-462

18. Sorensen LP, Sondergaard E, Nellemann B, Christiansen JS, Gormsen LC, Nielsen S: Increased VLDLtriglyceride secretion precedes impaired control of endogenous glucose production in obese, normoglycemic men. Diabetes 2011;60:2257-2264

19. Muris DM, Houben AJ, Schram MT, Stehouwer CD: Microvascular dysfunction: an emerging pathway in the pathogenesis of obesity-related insulin resistance. Reviews in endocrine \& metabolic disorders 2013;14:29-38

20. Mottillo S, Filion KB, Genest J, Joseph L, Pilote L, Poirier P, Rinfret S, Schiffrin EL, Eisenberg MJ: The metabolic syndrome and cardiovascular risk a systematic review and meta-analysis. Journal of the American College of Cardiology 2010;56:1113-1132

21. Marchesini G, Brizi M, Bianchi G, Tomassetti S, Bugianesi E, Lenzi M, McCullough AJ, Natale S, Forlani G, Melchionda N: Nonalcoholic fatty liver disease: a feature of the metabolic syndrome. Diabetes 2001; 50:1844-1850

22. Fabbrini E, Magkos F, Mohammed BS, Pietka T, Abumrad NA, Patterson BW, Okunade A, Klein S: Intrahepatic fat, not visceral fat, is linked with metabolic complications of obesity. Proc Natl Acad Sci U S A 2009;106:15430-15435

23. Kotronen A, Yki-Jarvinen H: Fatty liver: a novel component of the metabolic syndrome. Arterioscler Thromb Vasc Biol 2008;28:27-38

24. Farrell GC, Larter CZ: Nonalcoholic fatty liver disease: from steatosis to cirrhosis. Hepatology 2006;43: S99-S112

25. Matteoni CA, Younossi ZM, Gramlich T, Boparai N, Liu YC, McCullough AJ: Nonalcoholic fatty liver disease: a spectrum of clinical and pathological severity. Gastroenterology 1999;116:1413-1419

26. Argo CK, Caldwell SH: Epidemiology and natural history of non-alcoholic steatohepatitis. Clinics in liver disease 2009;13:511-531

27. Targher G, Day CP, Bonora E: Risk of cardiovascular disease in patients with nonalcoholic fatty liver disease. N Engl J Med 2010;363:1341-1350

28. Schindhelm RK, Dekker JM, Nijpels G, Bouter LM, Stehouwer CD, Heine RJ, Diamant M: Alanine aminotransferase predicts coronary heart disease events: a 10-year follow-up of the Hoorn Study. Atherosclerosis 2007;191:391-396

29. Ekstedt M, Franzen LE, Mathiesen UL, Thorelius L, Holmqvist M, Bodemar G, Kechagias S: Long-term follow-up of patients with NAFLD and elevated liver enzymes. Hepatology 2006;44:865-873

30. Ong JP, Pitts A, Younossi ZM: Increased overall mortality and liver-related mortality in non-alcoholic fatty liver disease. J Hepatol 2008;49:608-612

31. Fried SK, Bunkin DA, Greenberg AS: Omental and subcutaneous adipose tissues of obese subjects release interleukin-6: depot difference and regulation by glucocorticoid. J Clin Endocrinol Metab 1998; 83:847-850

32. Hotamisligil GS, Arner P, Caro JF, Atkinson RL, Spiegelman BM: Increased adipose tissue expression of tumor necrosis factor-alpha in human obesity and insulin resistance. J Clin Invest 1995;95:2409-2415

33. Xu H, Barnes GT, Yang Q, Tan G, Yang D, Chou CJ, Sole J, Nichols A, Ross JS, Tartaglia LA, Chen H: Chronic inflammation in fat plays a crucial role in the development of obesity-related insulin resistance. J Clin Invest 2003;112:1821-1830

34. Trayhurn P, Wood IS: Adipokines: inflammation and the pleiotropic role of white adipose tissue. $\mathrm{Br} \mathrm{J}$ Nutr 2004;92:347-355

35. Shoelson SE, Lee J, Goldfine AB: Inflammation and insulin resistance. J Clin Invest 2006;116:1793-1801

36. Schenk S, Saberi M, Olefsky JM: Insulin sensitivity: modulation by nutrients and inflammation. J Clin Invest 2008;118:2992-3002

37. Yudkin JS, Stehouwer CD, Emeis JJ, Coppack SW: C-reactive protein in healthy subjects: associations with obesity, insulin resistance, and endothelial dysfunction: a potential role for cytokines originating from adipose tissue? Arterioscler Thromb Vasc Biol 1999;19:972-978

38. Iozzo P: Viewpoints on the way to the consensus session: where does insulin resistance start? The adipose tissue. Diabetes Care 2009;32 Suppl 2:S168-173 
39. Nielsen S, Guo Z, Johnson CM, Hensrud DD, Jensen MD: Splanchnic lipolysis in human obesity. J Clin Invest 2004;113:1582-1588

40. Donnelly KL, Smith Cl, Schwarzenberg SJ, Jessurun J, Boldt MD, Parks EJ: Sources of fatty acids stored in liver and secreted via lipoproteins in patients with nonalcoholic fatty liver disease. J Clin Invest 2005;115:1343-1351

41. Samuel VT, Petersen KF, Shulman GI: Lipid-induced insulin resistance: unravelling the mechanism. Lancet 2010;375:2267-2277

42. Tanti JF, Jager J: Cellular mechanisms of insulin resistance: role of stress-regulated serine kinases and insulin receptor substrates (IRS) serine phosphorylation. Curr Opin Pharmacol 2009;9:753-762

43. Hotamisligil GS, Peraldi P, Budavari A, Ellis R, White MF, Spiegelman BM: IRS-1-mediated inhibition of insulin receptor tyrosine kinase activity in TNF-alpha- and obesity-induced insulin resistance. Science 1996;271:665-668

44. Senn JJ, Klover PJ, Nowak IA, Mooney RA: Interleukin-6 induces cellular insulin resistance in hepatocytes. Diabetes 2002;51:3391-3399

45. Krogh-Madsen R, Plomgaard P, Moller K, Mittendorfer B, Pedersen BK: Influence of TNF-alpha and IL-6 infusions on insulin sensitivity and expression of IL-18 in humans. Am J Physiol Endocrinol Metab 2006; 291:E108-114

46. Kruszynska YT, Olefsky JM, Frias JP: Effect of obesity on susceptibility to fatty acid-induced peripheral tissue insulin resistance. Metabolism 2003;52:233-238

47. Pradhan AD, Manson JE, Rifai N, Buring JE, Ridker PM: C-reactive protein, interleukin 6, and risk of developing type 2 diabetes mellitus. JAMA 2001;286:327-334

48. Bertoni AG, Burke GL, Owusu JA, Carnethon MR, Vaidya D, Barr RG, Jenny NS, Ouyang P, Rotter JI: Inflammation and the incidence of type 2 diabetes: the Multi-Ethnic Study of Atherosclerosis (MESA). Diabetes Care 2010;33:804-810

49. Mahendran Y, Cederberg H, Vangipurapu J, Kangas AJ, Soininen P, Kuusisto J, Uusitupa M, Ala-Korpela M, Laakso M: Glycerol and Fatty Acids in Serum Predict the Development of Hyperglycemia and Type 2 Diabetes in Finnish Men. Diabetes Care 2013;

50. Trayhurn $\mathrm{P}$, Wang $\mathrm{B}$, Wood IS: Hypoxia in adipose tissue: a basis for the dysregulation of tissue function in obesity? Br J Nutr 2008;100:227-235

51. Mehta NN, McGillicuddy FC, Anderson PD, Hinkle CC, Shah R, Pruscino L, Tabita-Martinez J, Sellers KF, Rickels MR, Reilly MP: Experimental endotoxemia induces adipose inflammation and insulin resistance in humans. Diabetes 2010;59:172-181

52. Furukawa S, Fujita T, Shimabukuro M, Iwaki M, Yamada Y, Nakajima Y, Nakayama O, Makishima M, Matsuda M, Shimomura I: Increased oxidative stress in obesity and its impact on metabolic syndrome. J Clin Invest 2004;114:1752-1761

53. Ozcan U, Cao Q, Yilmaz E, Lee AH, Iwakoshi NN, Ozdelen E, Tuncman G, Gorgun C, Glimcher LH, Hotamisligil GS: Endoplasmic reticulum stress links obesity, insulin action, and type 2 diabetes. Science 2004;306:457-461

54. Gaens KH, Stehouwer CD, Schalkwijk CG: Advanced glycation endproducts and its receptor for advanced glycation endproducts in obesity. Curr Opin Lipidol 2013;24:4-11

55. Dongiovanni P, Fracanzani AL, Fargion S, Valenti L: Iron in fatty liver and in the metabolic syndrome: a promising therapeutic target. J Hepatol 2011;55:920-932

56. Onat A, Can G, Rezvani R, Cianflone K: Complement C3 and cleavage products in cardiometabolic risk. Clin Chim Acta 2011;412:1171-1179

57. Garcia-Compean D, Jaquez-Quintana JO, Gonzalez-Gonzalez JA, Maldonado-Garza H: Liver cirrhosis and diabetes: risk factors, pathophysiology, clinical implications and management. World J Gastroenterol 2009;15:280-288 


\section{Chapter 2.1}

Study populations 
Chapter 2.1 


\section{Study population of individuals with (increased risk of) metabolic diseases: The CODAM study}

The studies in Chapters $\mathbf{3}$ and $\mathbf{4}$ of this thesis were conducted with data from an ongoing, prospective cohort study on, among others, the natural progression of insulin resistance and glucose tolerance: the Cohort on Diabetes and Atherosclerosis Maastricht (CODAM) study. In order to observe a reasonable progression of metabolic and vascular outcomes within the 7-year follow-up of this study, eligible individuals with an increased risk of cardiometabolic diseases were selected from a large ongoing cohort study (1-3). Participants were invited in a screening for the CODAM study if they were of Caucasian ethnicity, $>40$ years-old, and met at least one of the following criteria: positive family history (first degree) of type 2 diabetes (T2DM), history of gestational diabetes, $\mathrm{BMI} \geq 25 \mathrm{~kg} / \mathrm{m}^{2}$, use of antihypertensive drugs, postprandial glucose $\geq 6.0 \mathrm{mmol} / \mathrm{l}$ or glycosuria. A total of 2715 individuals without known T2DM were screened by a first oral glucose tolerance test (OGTT), yielding 226 individuals with newly diagnosed T2DM, 385 with impaired glucose metabolism (IGM), and 1889 with normal glucose metabolism (NGM) according to WHO 1999 criteria (4). All 226 individuals with newly diagnosed T2DM, 291 individuals with IGM, and a random selection of 728 individuals with NGM were invited to participate in the CODAM study. To ensure participation of sufficient individuals with T2DM in the study, a sample of 134 patients with recently diagnosed T2DM was also invited. Eventually, a total of 574 individuals participated in the CODAM study (42\% participation rate), of whom 146 had T2DM (80 newly diagnosed, and 66 recently diagnosed), 127 IGM, and 301 NGM on the basis of a second OGTT. Mean age was $59 \pm 6.9$ years, $62 \%$ was male, and mean body mass index was $28.5 \pm 4.3 \mathrm{~kg} / \mathrm{m}^{2}$.

These 574 individuals were extensively characterized with regard to their lifestyle, and cardiovascular and metabolic profile during two visits to the University's metabolic research unit at the baseline evaluation of the study in 1999-2001. Participants handed in questionnaires on lifestyle factors such as alcohol, smoking, diet, physical activity, and on health status $(5,6)$. Moreover, adiposity measures, blood pressure, and several vascular measurements were obtained. A second OGTT was performed, and venous blood samples were taken for assessment of a variety of blood parameters, including glucose, insulin, lipids, and markers of inflammation. After a median of 7.0 years [IQR 6.9-7.1], 495 individuals participated in the first follow-up measurements (attrition rate 14\%), taking place from 2006 until 2009. Again during two visits to the research unit, the majority of measurements performed at baseline were repeated at follow-up. 


\section{Study population of patients with liver cirrhosis}

The studies in Chapter $\mathbf{5}$ of this thesis were conducted with retrospective data from a large series of patients with liver cirrhosis from the Catharina Hospital Eindhoven. Patients were selected by searching the digital diagnosis-treatment combination registry for codes "compensated liver cirrhosis" and "decompensated liver cirrhosis". We selected all patients treated for cirrhosis from January 2001 till December 2011. From the medical files and the (digital) laboratory information system of the Catharina Hospital, we collected several clinical and laboratory parameters related to liver cirrhosis and T2DM, using the first day of admission or the first contact at the outpatient clinic as baseline. The presence of T2DM at baseline was defined on the medical history and WHO 1999 criteria (4). Follow-up of patients was performed until May 2012 by checking (digital) medical files of all specialities in our hospital for relevant outcomes. 


\section{References}

1. Kruijshoop M, Feskens EJ, Blaak EE, de Bruin TW: Validation of capillary glucose measurements to detect glucose intolerance or type 2 diabetes mellitus in the general population. Clin Chim Acta 2004; 341:33-40

2. van Dam RM, Grievink L, Ocke MC, Feskens EJ: Patterns of food consumption and risk factors for cardiovascular disease in the general Dutch population. Am J Clin Nutr 2003;77:1156-1163

3. van Dam RM, Boer JM, Feskens EJ, Seidell JC: Parental history of diabetes modifies the association between abdominal adiposity and hyperglycemia. Diabetes Care 2001;24:1454-1459

4. WHO: Definition, diagnosis and classification of diabetes mellitus. Report of a WHO Consultation. Part 1: Diagnosis and classification of diabetes mellitus. In WHO/NCD/NCS/992 Geneva, Switzerland, 1999

5. Wendel-Vos GC, Schuit AJ, Saris WH, Kromhout D: Reproducibility and relative validity of the short questionnaire to assess health-enhancing physical activity. J Clin Epidemiol 2003;56:1163-1169

6. Ocke MC, Bueno-de-Mesquita HB, Goddijn HE, Jansen A, Pols MA, van Staveren WA, Kromhout D: The Dutch EPIC food frequency questionnaire. I. Description of the questionnaire, and relative validity and reproducibility for food groups. Int J Epidemiol 1997;26 Suppl 1:S37-48 


\section{Chapter}

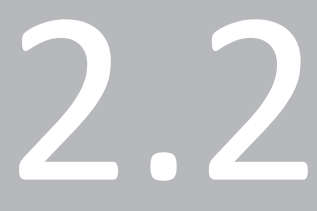

The use of calibrated data in prospective studies 
Chapter 2.2 
Some of the biomarkers that were obtained in the Cohort on Diabetes and Atherosclerosis Maastricht (CODAM) study, i.e. fasting insulin, non-esterified fatty acids (NEFAs), complement factor 3 (C3), and the inflammatory markers interleukin-6 (IL-6), high sensitivity C-reactive protein (hs-CRP), serum amyloid $A$ (SAA), and soluble inter-cellular adhesion molecule-1 (sICAM-1) were measured initially at the time the baseline evaluation of the CODAM study was completed (i.e. 1999-2001). More recently (2010-2012), paired (re-) measurements of these biomarkers at baseline and follow-up were performed. As such, these markers were measured twice at baseline. To make use of all available data and to increase the precision of our estimated effect sizes, we intended to use the mean of both baseline measurements for our analyses.

Some markers at baseline were determined twice by the exact same method (e.g. NEFAs), and hence showed very good absolute agreement (intraclass correlation coefficient (ICC) 0.96) and no systematic differences. As such, the mean of the two measurements at baseline could be taken for further analyses. However, some biomarkers at baseline were measured twice, but using two different methods: e.g. fasting insulin levels were determined by two-sided immunoradiometric test (IRMA) in 2001 and by multi-array (MA) detection system based on electrochemiluminicence technology in 2012. Consequently, the two different measurements of fasting insulin at baseline showed moderate agreement (ICC 0.83) and a systematic difference of $21 \mathrm{pmol} / \mathrm{l}$ (95\% limits of agreement (LoA) -37 to $80 \mathrm{pmol} / \mathrm{l}$ ). Moreover, the measurements of fasting insulin by IRMA in 2001 could not directly be compared with measurements of fasting insulin by MA in 2012.

In order to use all available data and to ensure comparability of baseline and followup fasting insulin levels, the previous baseline measurements of fasting insulin (IRMA) were calibrated (i.e. converted by means of a regression equation) to the multi-array measurements using Deming regression models as recommended for method comparison (1,2). After calibration of the IRMA measurements to the MA measurements, the two insulin variables showed very good agreement (ICC 0.96), there was no systematic difference, the $95 \%$ LoA were narrower (-40 to $40 \mathrm{pmol} / \mathrm{l})$, and calibrated fasting insulin levels were directly comparable to the MA measurement of fasting insulin at follow-up. After this calibration, the mean of the two fasting insulin measurements at baseline could be used for further analyses (Chapters $\mathbf{3 . 3}$ and 4.3). Analogously, calibration equations were used for complement factor 3 (C3) (Chapter 4.3), and the inflammatory markers IL-6, hs-CRP, SAA, and SICAM-1 (Chapters 3.3 and 4.3). 


\section{References}

1. Martin RF: General deming regression for estimating systematic bias and its confidence interval in method-comparison studies. Clin Chem 2000;46:100-104

2. van Bussel BC, Ferreira I, van de Waarenburg MP, van Greevenbroek MM, van der Kallen CJ, Henry RM, Feskens EJ, Stehouwer CD, Schalkwijk CG: Multiple inflammatory biomarker detection in a prospective cohort study: a cross-validation between well-established single-biomarker techniques and an electrochemiluminescense-based multi-array platform. PLoS One 2013;8:e58576; DOI 10.1371/journal.pone.0058576 


\section{Chapter 2}

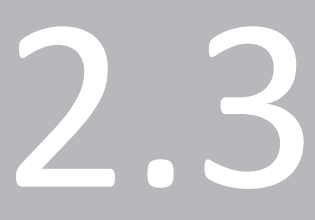

Measures of glucose tolerance, insulin resistance, inflammation, hepatocellular damage, and liver fat 
Chapter 2.3 


\section{Measures of glucose tolerance and insulin resistance}

In the Cohort on Diabetes and Atherosclerosis Maastricht (CODAM) study, a standard $75 \mathrm{~g}$ oral glucose tolerance test (OGTT) was performed with measurement of glucose levels at 0, 30, 60 and $120 \mathrm{~min}$, and, during the follow-up round, also at $15 \mathrm{~min}$. More recently, we performed paired measurements of plasma insulin levels, at baseline and follow-up, at all OGTT time-points mentioned above. Total area under the curves (AUC) for glucose and insulin during the OGTT (for the first 30 minutes and for 120 minutes) were calculated using the trapezoidal method.

Throughout this thesis, we used fasting glucose levels, $2 \mathrm{~h}$ glucose levels, and $A \cup C_{\text {glucose }}$ as measures of glucose tolerance. Moreover, we used several measures of insulin resistance. HOMA2-IR, which has been shown to correlate well with (muscle) glucose disposal in clamp studies $(r=0.7-0.8) \quad(1,2)$, was computed using the HOMA2-calculator considering fasting plasma glucose and specific insulin data (http://www.dtu.ox.ac.uk/homacalculator/index.php). In addition to the commonly used HOMA2-IR, we also calculated two organ-specific measures reflecting insulin resistance in the liver and in adipocytes. Hepatic insulin resistance (hepatic IR) was estimated using the square root of the product of the areas under the curve for glucose and insulin during the first 30 minutes of the OGTT (i.e. SQRT(glucose $e_{0-30}[A U C] \times$ insulin $_{0-30}$ [AUC])) converted to conventional units $([\mathrm{mg} / \mathrm{dl} \cdot \mathrm{hr}] \times[\mathrm{ulU} / \mathrm{ml} \cdot \mathrm{hr}])$. This index has been developed and validated against the product of fasting plasma insulin and endogenous glucose production in clamp studies $(r=0.6)$, and has been postulated to represent hepatic insulin resistance, as it not only takes into account fasting glucose, but also the early suppression of glucose by insulin during the first phase of the OGTT $(3,4)$. Finally, adipocyte insulin resistance (adipocyte IR) was calculated as the product of fasting insulin and fasting non-esterified fatty acid (NEFA) concentrations, as described before $(5,6)$. This index provides a valid marker of the sensitivity of adipocytes to the antilipolytic effect of insulin, since NEFA concentrations and fatty acid turnover in clamp studies are highly correlated, and insulin suppresses lipolysis already at low (i.e. fasting) concentrations (10-20 $\mathrm{mU} / \mathrm{l})(6,7)$.

\section{Measures of inflammation}

In the CODAM cohort, several markers of systemic low-grade inflammation (LGI) were measured. In the baseline evaluation (cross-sectional studies), these markers included high sensitivity C-reactive protein (hs-CRP), haptogobin, ceruloplasmin, interleukin-6 (IL-6), serum amyloid A (SAA), and soluble inter-cellular adhesion molecule-1 (sICAM-1) (8). In the longitudinal studies, paired multi-array measurements of a slightly different set of six markers were used, including IL-6, hs-CRP, SAA, SICAM-1, and now also interleukin-8 (IL-8) and tumour necrosis factor- $\alpha$ (TNF- $\alpha$ ) (9). In several studies in the 
present thesis, we used a general low-grade inflammation (LGI) score that integrated these markers into one single composite score (10).

First, all inflammatory markers were In transformed to obtain a normal distribution. Next, the population mean and standard deviation were calculated for each In transformed marker. Then, for each individual subject in the study, these In markers were transformed into Z-scores (i.e. standardized values) by subtracting the population mean from the individuals' observed value and dividing by the standard deviation (i.e. individuals' observed value - population mean) /SD). Finally, for each person, the individual inflammatory Z-scores were averaged to obtain a single, robust measure of LGI. This composite score was used to provide a robust measure of low-grade inflammation, as it can be interpreted as six repeated measurements of the same construct (namely inflammation), thereby reducing measurement error. The use of such a score also reduces the influence of biological variability of each marker if assessed separately, and prevents multiple testing problems. A possible drawback of averaging inflammatory markers into a score is that it does not take into account potential differences in biological importance of individual markers, but instead focuses on the overall effect of the inflammatory state. At the same time, value lies in the fact that this approach prevents that too much emphasis is laid on the individual inflammatory marker that, possibly by chance or by differences in measurement error, showed the strongest individual effect, when in fact it is the underlying general inflammation process that is most relevant.

\section{Measures of liver fat and hepatocellular damage}

In the CODAM study, we measured three markers of hepatocellular injury in blood: aspartate amino transferase (AST), alanine aminotransferase (ALT), and gamma-glutamyl transferase (GGT). Analogous to the general score for inflammation, we used a combined liver enzyme score (LE score) as one integrated measure of hepatocellular injury by averaging the Z-scores [i.e. (individuals' observed values population mean)/SD] of AST, ALT, and GGT. In addition, we estimated liver fat content (eLF\%) with the use of a predictive equation that has recently been developed and validated against proton magnetic resonance spectroscopy $\left({ }^{1} \mathrm{H}-\mathrm{MRS}\right)$, and provides an inexpensive tool for epidemiological research (11). This equation estimates liver fat content on the basis of data on liver enzymes (AST and AST/ALT ratio), fasting insulin, presence of the metabolic syndrome (IDF definition) and presence of type 2 diabetes mellitus. This equation correlated well with liver fat content on MRS ( $r=0.70)$, and showed an area under receiver operating curve of $0.86(95 \% \mathrm{Cl} 0.80-0.92)$ in predicting a liver fat content $>5.56 \%$, which is considered the threshold for (non-alcoholic) fatty liver disease (12). 


\section{References}

1. Wallace TM, Levy JC, Matthews DR: Use and abuse of HOMA modeling. Diabetes Care 2004;27: 1487-1495

2. Bonora E, Targher G, Alberiche M, Bonadonna RC, Saggiani F, Zenere MB, Monauni T, Muggeo M: Homeostasis model assessment closely mirrors the glucose clamp technique in the assessment of insulin sensitivity: studies in subjects with various degrees of glucose tolerance and insulin sensitivity. Diabetes Care 2000;23:57-63

3. Abdul-Ghani MA, Matsuda M, Balas B, DeFronzo RA: Muscle and liver insulin resistance indexes derived from the oral glucose tolerance test. Diabetes Care 2007;30:89-94

4. Vangipurapu J, Stancakova A, Kuulasmaa T, Paananen J, Kuusisto J, Ferrannini E, Laakso M: A novel surrogate index for hepatic insulin resistance. Diabetologia 2011;54:540-543

5. Vangipurapu J, Stancakova A, Pihlajamaki J, Kuulasmaa TM, Kuulasmaa T, Paananen J, Kuusisto J, Ferrannini E, Laakso M: Association of indices of liver and adipocyte insulin resistance with 19 confirmed susceptibility loci for type 2 diabetes in 6,733 non-diabetic Finnish men. Diabetologia 2011; 54:563-571

6. Abdul-Ghani MA, Molina-Carrion M, Jani R, Jenkinson C, Defronzo RA: Adipocytes in subjects with impaired fasting glucose and impaired glucose tolerance are resistant to the anti-lipolytic effect of insulin. Acta Diabetol 2008;45:147-150

7. Groop LC, Bonadonna RC, DelPrato S, Ratheiser K, Zyck K, Ferrannini E, DeFronzo RA: Glucose and free fatty acid metabolism in non-insulin-dependent diabetes mellitus. Evidence for multiple sites of insulin resistance. J Clin Invest 1989;84:205-213

8. Jacobs M, van Greevenbroek MM, van der Kallen CJ, Ferreira I, Blaak EE, Feskens EJ, Jansen EH, Schalkwijk CG, Stehouwer CD: Low-grade inflammation can partly explain the association between the metabolic syndrome and either coronary artery disease or severity of peripheral arterial disease: the CODAM study. Eur J Clin Invest 2009;39:437-444

9. van Bussel BC, Ferreira I, van de Waarenburg MP, van Greevenbroek MM, van der Kallen CJ, Henry RM, Feskens EJ, Stehouwer CD, Schalkwijk CG: Multiple inflammatory biomarker detection in a prospective cohort study: a cross-validation between well-established single-biomarker techniques and an electrochemiluminescense-based multi-array platform. PLoS One 2013;8:e58576; DOI 10.1371/journal.pone.0058576

10. van Bussel BC, Henry RM, Schalkwijk CG, Ferreira I, Feskens EJ, Streppel MT, Smulders YM, Twisk JW, Stehouwer CD: Fish consumption in healthy adults is associated with decreased circulating biomarkers of endothelial dysfunction and inflammation during a 6-year follow-up. J Nutr 2011;141:1719-1725

11. Kotronen A, Peltonen M, Hakkarainen A, Sevastianova K, Bergholm R, Johansson LM, Lundbom N, Rissanen A, Ridderstrale M, Groop L, Orho-Melander M, Yki-Jarvinen H: Prediction of non-alcoholic fatty liver disease and liver fat using metabolic and genetic factors. Gastroenterology 2009;137:865-872

12. Szczepaniak LS, Nurenberg P, Leonard D, Browning JD, Reingold JS, Grundy S, Hobbs HH, Dobbins RL: Magnetic resonance spectroscopy to measure hepatic triglyceride content: prevalence of hepatic steatosis in the general population. Am J Physiol Endocrinol Metab 2005;288:E462-468 


\section{Chapter

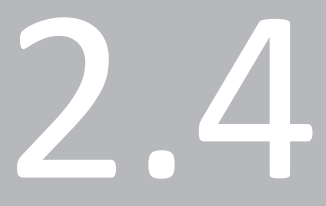

Interpretation of longitudinal

data analyses 
Chapter 2.4 
The present thesis presents data of both cross-sectional and longitudinal evaluations of observational cohort data. Some particular aspects of analyses using data that are obtained at multiple points in time (follow-up data) are discussed in this chapter. Two prospective analyses were conducted with data from the Cohort on Diabetes and Atherosclerosis Maastricht (CODAM) study, one using a prospective (Chapter 3.3) and one using a longitudinal (Chapter 4.3) approach. In addition, one retrospective cohort study on survival of patients with liver cirrhosis was performed (Chapter 5.3). In each of these studies, the datasets differed with respect to their structure, and therefore needed different statistical analyses. Given this diversity on statistical approaches on cohort data, a brief description of these analyses and interpretation of the obtained regression coefficients is given here, using examples of hypothetical associations between waist circumference $(\mathrm{cm})$ and fasting glucose levels $(\mathrm{mmol} / \mathrm{l})$.

\section{Prospective associations}

In a prospective cohort study, most determinants (in this example waist) are measured only at baseline, while the outcome (in this example fasting glucose) is measured at baseline and during follow-up (Figure 2.4.1; panel A). In this situation, there are several ways to analyse the association between waist and fasting glucose levels, depending on the use of continuous and/or dichotomous variables.

First, the association between waist (at baseline) and glucose (both baseline and follow-up values) over the whole follow-up period can be investigated using generalized estimating equations (GEE) to account for the correlation of repeated measurements of glucose within individuals. In these analyses, a regression coefficient of e.g. $\beta=0.15$ can be interpreted as follows: per $1 \mathrm{~cm}$ difference in baseline waist circumference between individuals, glucose levels are $0.15 \mathrm{mmol} / \mathrm{l}$ higher over the whole follow-up period (Figure 2.4.1A; paths a and b). This technique makes use of all available data in the study.

Second, one may investigate whether baseline waist is associated with changes (absolute or relative to baseline) in glucose levels during follow-up (Figure 2.4.1A; path c). In such analyses, a regression coefficient of e.g. $\beta=0.02$ means that per $1 \mathrm{~cm}$ difference in baseline waist circumference between individuals, the change in glucose levels (within individuals) during the follow-up period is $0.02 \mathrm{mmol} / \mathrm{l}$ higher. When the outcome (glucose) is only measured twice (i.e. at baseline and follow-up), such analyses can be performed with simple linear regression. This technique provides more insight into within-subject effects, but is restricted to individuals attending follow-up, and hence susceptible to selective attrition. Of note, sometimes change analyses are adjusted for baseline values of the outcome (so-called autoregressive analyses), but such analyses may lead to inflation of the changes (within-subject) coefficients, and biased, spurious associations between determinants and changes in outcomes (1). 
A)

$$
\begin{aligned}
& \text { Baseline } \\
& \text { Waist circumference }
\end{aligned}
$$

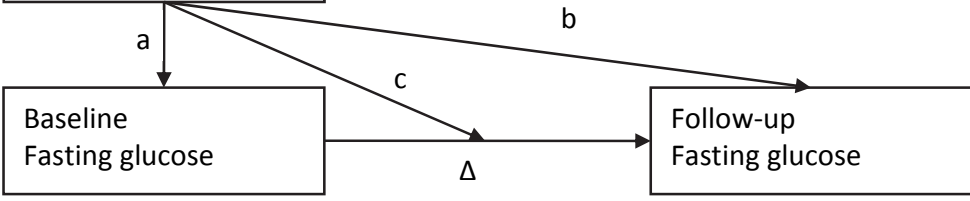

B)

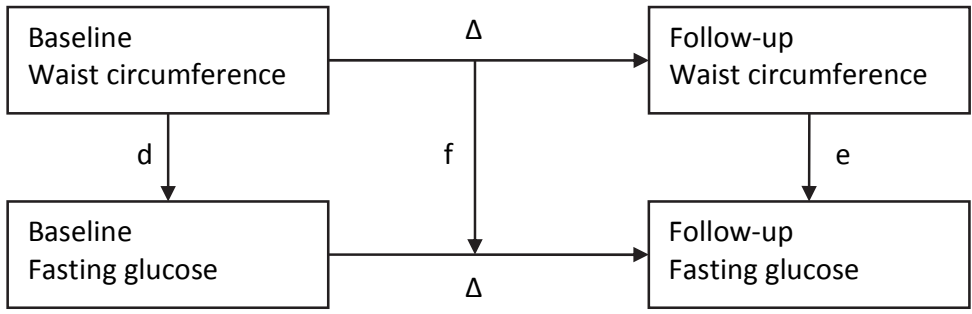

Figure 2.4.1 Relationships between measures of determinant (waist circumference) and outcome (fasting glucose) in prospective (A) and longitudinal (B) study designs

Third, the association between waist and incident type 2 diabetes mellitus (T2DM) can be investigated if glucose levels are dichotomized into 'no T2DM' and 'T2DM', and individuals with T2DM at baseline are excluded from the analyses. These data can be analysed using logistic regression analyses, using waist at baseline as determinant, and the cumulative incidence of T2DM at follow-up as outcome. In these analyses, an odds ratio (OR) of e.g. 1.2 can be interpreted as follows: per $1 \mathrm{~cm}$ higher waist circumference between individuals at baseline, the odds to develop incident T2DM during follow-up are 1.2 times higher. Similar to the previous analysis, this technique is restricted to the individuals that attend follow-up and hence susceptible to selective attrition. The use of dichotomized data may, on the one hand, lead to loss of information, but, on the other hand, provide data on associations with clinically relevant outcomes instead of intermediate measures.

Fourth, if glucose levels were measured on a frequent basis (e.g. every 3 months), the association between waist and incident T2DM could be examined accounting for the selective attrition and loss to follow-up by censoring at the last available glucose measurement. In that case, survival analysis using Cox proportional hazards models could be used to investigate the association between baseline waist circumference and (the hazard of) T2DM during follow-up. In such analyses, a hazard ratio (HR) of e.g. 1.2 means that per $1 \mathrm{~cm}$ difference in baseline waist circumference between individuals, 
the hazard to develop T2DM at any time during follow-up is 1.2 times higher. These analyses are often used for outcomes such as mortality or clinical outcomes (see Chapter 5.3), for which no extensive monitoring is necessary.

\section{Longitudinal associations}

In a longitudinal study, not only the outcome (glucose) is measured at baseline and follow-up, but the determinant (waist) is also measured multiple times (Figure 2.4.1; panel B). The availability of such data allows the application of more or less the same kind of analyses as presented above, although the interpretation is somewhat different.

The overall longitudinal association between waist and fasting glucose over the whole follow-up period can be investigated with GEE in all individuals. The coefficients of this GEE analysis can be interpreted as a combination of both between-subject effects and within-subject effects over time. A regression coefficient of e.g. $\beta=0.25$ may on the one extreme mean that per $1 \mathrm{~cm}$ difference in waist between individuals, fasting glucose is generally $0.25 \mathrm{mmol} / \mathrm{l}$ higher over the follow-up period, potentially without any within-subject effect (Figure 2.4.1B; paths $d$ and e). At the other extreme, the coefficient may reflect that per $1 \mathrm{~cm}$ increase in waist within individuals during the follow-up, glucose levels in those individuals increase, on average, by $0.25 \mathrm{mmol} / \mathrm{I}$ (Figure 2.4.1B; path f). In reality, the regression coefficient represents a combination of these two scenarios, thus representing the longitudinal association between waist and glucose over the follow-up period, with no indication of the relative contribution of the between- and the within-subject effects.

To address specifically the within- component of this association, one may investigate whether changes in waist are associated with changes in glucose levels during follow-up (Figure 2.4.1B; path $\mathrm{f}$ ). In the case of only two time-points, this analysis can be performed with simple linear regression. In such analyses, a regression coefficient of e.g. $\beta=0.05$ means that per $1 \mathrm{~cm}$ greater change in waist between individuals, the change in fasting glucose levels (within individuals) during the follow-up period is $0.05 \mathrm{mmol} / \mathrm{l}$ higher. Again, this technique provides more insight into withinsubject effects, but is restricted to individuals attending follow-up. 


\section{References}

1. Glymour MM, Weuve J, Berkman LF, Kawachi I, Robins JM: When is baseline adjustment useful in analyses of change? An example with education and cognitive change. Am J Epidemiol 2005;162: 267-278 


\section{Chapter}

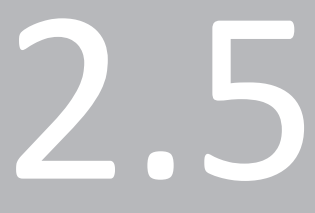

Mediation analyses in aetiological biomedical research: The influence of random error

Nick Wlazlo, Bas C.T. van Bussel, Marleen MJ van Greevenbroek, Casper G Schalkwijk, Bert Bravenboer, Isabel Ferreira, Coen DA Stehouwer 


\section{Abstract}

The application of statistical mediation analyses using markers of intermediary pathophysiological processes and subclinical disease enables the study of complex aetiological mechanisms in epidemiological studies. However, these analytical techniques have mostly been developed and applied in the social sciences, and their use in biomedical research is yet scarce. In some recent (mediation) studies, we were confronted with the question how random error in the mediator variable and the other variables may affect the results and conclusions of mediation analysis. In this paper, we provide a brief overview of mediation analysis, and show by both a simulation study and a practical example that with increasing random error of the mediator, the size of the mediated effect decreases and eventually becomes non-significant. This is particularly relevant when a mediator is measured with less precision compared to the proximal causal factor, leading to a striking attenuation of the independent association of a mediator with the outcome. Researchers performing mediation analyses should therefore always specify the presumed a priori causality, and consider both confounding and the role of random error when crude associations are attenuated after adjustment for potential proximal causal factors. 


\section{Introduction to mediation analysis}

Epidemiological research on aetiology in medicine and health sciences has always focused on examining independent associations between risk factors and (markers) of disease. Although establishing the correlation between two variables is a necessary first step towards claiming a causal relation, it is even of equally great scientific interest to investigate how or by what pathophysiological processes a risk factor leads to disease. Major technical advances in biomedical research over the past decades have provided us with the tools to examine such intermediary pathophysiological processes, for example by using biomarkers, imaging techniques, scintigraphy, or function tests (e.g. spirometry, flow-mediated vasodilation, glucose clamp studies, etc). Hypotheses about the role of intermediary variables should be established on theoretical grounds and a priori causal evidence (1-3), and can statistically be investigated using mediation analyses, which have been extensively described in psychological and behavioural science (2-6).

Traditionally, mediation is said to occur when (part of) the overall association between a determinant $(X)$ and outcome $(Y)$ is explained by (i.e. attenuated after adjustment for) a hypothesized intervening variable: mediator $M$ (3). Several approaches are available to test mediation, but, according to recent recommendations, the best approach is to estimate the absolute value of the mediated effect and the $95 \%$ confidence interval $(95 \% \mathrm{Cl})$ around it $(4,6,7)$. The causal diagram in Figure 2.5 .1 shows how this is done for the situation with one mediator, called simple mediation, but more complex models with multiple mediators are also possible (2). Panel A shows the total or overall (causal) effect of $X$ on $Y$, called path $c$. Panel $B$ shows the role of the intervening mediator $M$. Path a represents the effect of $X$ on $M$, path $b$ represents the effect of $M$ on $Y$ independent of $X$, and path $c^{\prime}$ is the direct effect of $X$ on $Y$ independent of $\mathrm{M}$. The effect of $\mathrm{X}$ on $\mathrm{Y}$ that is mediated through $\mathrm{M}$, called the indirect or mediated effect, can be quantified by the product of regression coefficients $a$ and $b$, or $a b$. When all variables are measured on a continuous scale, the total effect of $X$ on $Y$ (c) can be apportioned mathematically into the mediated effect through $M(a b)$ and the direct effect of $X$ on $Y\left(c^{\prime}\right)$ (i.e. $\left.c=c^{\prime}+a b\right)$. Since the product $a b$ is asymmetrically distributed, the $95 \% \mathrm{Cl}$ around this effect should not be derived from normal theory, but from the empirical distribution by bootstrapping the product ab (5-7). It should be noted, that, statistically, mediation and confounding are identical and cannot be differentiated, stressing the importance of having a priori causal hypotheses before analyzing data.

The application of these mediation analyses in biomedical epidemiological research is as yet limited (8). In the present thesis, we showed in several studies that these analyses are able to provide a comprehensive approach to gain more insight in underlying mechanisms in metabolic research (Chapters 3.2, 4.2, and 4.4) (9-11). In these studies however, we were also confronted with a possible limitation of mediation analysis when the mediator was measured with (random) error. This leads to an 
underestimation of its associations with $\mathrm{X}$ and $\mathrm{Y}$ (also called regression dilution bias) (12), and hence an underestimation of mediation. In the statistical literature, this is regarded only as a methodological artefact, and not as a theoretical problem $(6,13)$. In biomedical epidemiology, however, random error is a common problem that cannot simply be ignored $(12,14)$. Analytical measurement error (i.e. the precision of the measurement) and biological variability (i.e. real but temporary deviations from an individual's usual value) contribute to this random error, particularly with regard to risk factors such as blood pressure, dietary characteristics, physical activity, amounts of cigarettes smoked, alcohol intake, blood glucose and cholesterol, and biomarkers $(12,14)$. Multiple or repeated measurements may reduce the influence of random error considerably, but most observational studies measure risk factors only on a single occasion (i.e. at baseline) (12). Random error in both determinant (X) and mediator (M) may attenuate their associations with outcome (Y), and therefore remains an important problem in biomedical research. Random error in the outcome (Y) decreases the precision of estimates, but usually does not affect unstandardized effect sizes.

To examine the influence of such random error on the results and conclusions of mediation analysis under controlled conditions, we performed a simulation study with a fictional database. In addition, we illustrate the influence of random error in a real dataset using three biomarkers with increasing analytical measurement error (reflected by a larger interassay coefficient of variation) as mediators.

\section{Simulation study}

To study the influence of random error under controlled circumstances, we created a plausible epidemiological database for $n=1000$ using the fictional causal associations shown in Figure 2.5.1. The genuine regression coefficient for the association between determinant $X$ and outcome $Y$ was set to be $\beta=20$ (path $c$ ), and this could be apportioned into a direct effect of $X$ on $Y$ with $\beta=5$ (path $c^{\prime}$ ) and an indirect or mediated effect of $X$ through mediator $M$ on $Y$ with $\beta=15$ (path ab: $3 \cdot 5=15$ ). In other words, $75 \%$ of the effect of $X$ on $Y$ is said to be mediated through (or caused by) M. We created three variables with increasing random error (indicated by numbers) for both $X, M$ and $Y$ by adding random variation to the variables (Table 2.5.1). First, we added a small amount of random variation to the calculated variables $\mathrm{m} 1$ and $\mathrm{y} 1$ in order to simulate a realistic biomedical database. Second, variables $x 2, x 3, m 2, m 3, y 2$ and y3 were created by adding substantially more random variation.

Simple mediation analyses were performed to assess the magnitude of mediation by $M$ in the associations between $X$ and $Y$ for all combinations of variables (2). Regression coefficients and $95 \%$ confidence intervals $(\mathrm{Cl})$ of individual paths $a, b, c$ and $c^{\prime}$ in Figure 2.5.1 were derived from linear regression analyses. The magnitude of the 
mediated effect by $\mathrm{M}$ was calculated as the product of paths $\mathrm{a}$ and $\mathrm{b}$ (product $\mathrm{ab}$ ), and asymmetric (bias-corrected) $95 \% \mathrm{Cls}$ around this effect were estimated by bootstrapping (5000 samples) (2). Analyses were performed using the Statistical Package for Social Sciences for Windows, version 17.0 (SPSS Inc., Chicago, IL, USA).

A

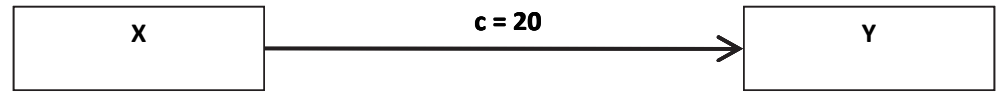

B

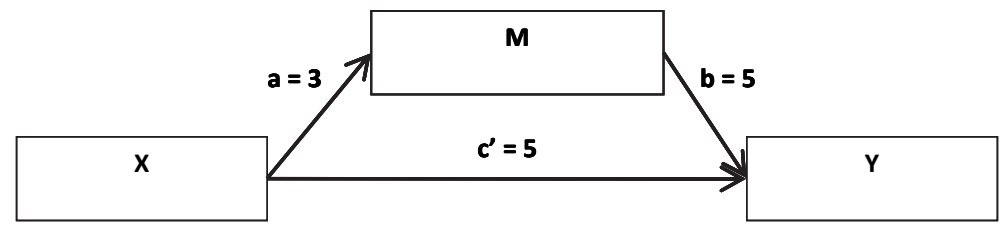

$$
\begin{aligned}
\mathrm{Y} & =5 \cdot \mathrm{M}+5 \cdot \mathrm{X} \\
& =15 \cdot \mathrm{X}+5 \cdot \mathrm{X} \\
& =20 \cdot \mathrm{X}
\end{aligned}
$$

Figure 2.5.1 Causal diagrams for mediation analysis.

Panel $A$ shows the association between a determinant $X$ and outcome $Y$. Path $c$ represents the overall association between $X$ and $Y$. Panel $B$ shows how the role of a single mediator is assessed in simple mediation analysis. Mediation is said to occur when (part of) the overall association between $X$ and $Y$ is explained by (i.e. attenuated after adjustment for) a hypothesized intervening variable: mediator $M$. The product of regression coefficients $a$ and $b$ (ab) quantifies the indirect or mediated effect by $M$ in the relation between $X$ and $Y$. The part of the association not explained by mediator $M$ is the direct effect of $X$ on $Y$ and is represented by

\begin{tabular}{|c|c|c|c|c|c|}
\hline & Mean & SD & Minimum & Maximum & SPSS formula \\
\hline $\mathrm{x} 1$ & 27.90 & 4.08 & 15.92 & 41.19 & x1=RV.NORMAL $(28,4)$ \\
\hline$x 2$ & 27.94 & 4.57 & 12.71 & 42.69 & $x 2=x 1+R V \cdot \operatorname{NORMAL}(0,2)$ \\
\hline$x 3$ & 27.85 & 6.39 & 6.32 & 52.67 & $x 3=x 1+R V \cdot \operatorname{NORMAL}(0,5)$ \\
\hline $\mathrm{m} 1$ & 83.74 & 13.58 & 38.73 & 126.46 & $\mathrm{~m} 1=3 * x 1+\mathrm{RV} \cdot \mathrm{NORMAL}(0,5)$ \\
\hline $\mathrm{m} 2$ & 84.25 & 15.68 & 32.30 & 141.36 & $m 2=3 * x 1+R V \cdot \operatorname{NORMAL}(0,10)$ \\
\hline m3 & 84.28 & 23.04 & 6.15 & 156.19 & $m 3=3 * x 1+R V \cdot \operatorname{NORMAL}(0,20)$ \\
\hline y1 & 557.98 & 102.82 & 244.27 & 887.97 & $y 1=5^{*} m 1+5^{*} x 1+R V \cdot \operatorname{NORMAL}(0,50)$ \\
\hline y2 & 562.36 & 128.55 & 121.37 & 974.24 & $y 2=5^{*} m 1+5^{*} x 1+R V \cdot \operatorname{NORMAL}(0,100)$ \\
\hline y3 & 560.83 & 167.98 & 36.98 & 1048.52 & $y 3=5^{*} m 1+5^{*} x 1+R V \cdot \operatorname{NORMAL}(0,150)$ \\
\hline
\end{tabular}
path $c^{\prime}$. When all variables are measured on a continuous scale, the sum of $a b$ and $c^{\prime}$ equals $c$.

Table 2.5.1 Characteristics of the simulation dataset. 


\section{Results}

The influence of increasing levels of random error on the results of the mediation analysis is shown in Table 2.5.2. As expected, the estimates for paths $c, c^{\prime}$ and ab using variables $\mathrm{x} 1, \mathrm{~m} 1$ and $\mathrm{y} 1$ approximated the true associations depicted in Figure 2.5.1. The magnitude of the mediation in absolute values was $\beta=15.17$ (95\% $\mathrm{Cl} 13.36-17.24)$. However, when mediators $\mathrm{m} 2$ and $\mathrm{m} 3$ were used, the magnitude of the mediated effects decreased substantially as shown across Table 2.5.2. Although bootstrapping of asymmetric $95 \% \mathrm{Cls}$ still detected significant mediation, there were also cases in which the mediated effect $(a b)$ was no longer statistically significant. We show that this was particularly true when a mediator with more random error was combined with a relatively precise determinant $X$, such as combinations $x 1-m 2, x 1-m 3$ and $x 2-m 3$. On the other hand, combination of an imprecise determinant $(x 3)$ with relatively precise mediator $(m 1)$ revealed complete mediation with no remaining direct effect of $X$ on $Y$ (paths $c^{\prime}$ ). Finally, increasing random error in the outcome (Y) did not affect unstandardized regression coefficients, but only decreased precision of the estimates, indicated by larger $95 \% \mathrm{Cls}$.

Table 2.5.2 Influence of measurement error in determinant, mediator and outcome on the results of mediation analysis.

\begin{tabular}{|c|c|c|c|c|c|c|c|c|c|c|}
\hline & & & y1 & & & y2 & & & y3 & \\
\hline & & path§ & $\beta$ & $95 \% \mathrm{Cl}$ & path§ & $\beta$ & $95 \% \mathrm{Cl}$ & path§ & $\beta$ & $95 \% \mathrm{Cl}$ \\
\hline$x 1$ & $\mathrm{~m} 1$ & c & 20.93 & $20.06 ; 21.80$ & c & 19.78 & $18.25 ; 21.30$ & c & 20.55 & $18.34 ; 22.77$ \\
\hline & & $c^{\prime}$ & 5.75 & $3.71 ; 7.80$ & $c^{\prime}$ & 6.93 & $3.04 ; 10.83$ & $c^{\prime}$ & 7.89 & $2.15 ; 13.64$ \\
\hline & & $a b$ & 15.17 & $13.36 ; 17.24$ & $a b$ & 12.84 & $9.44 ; 16.48$ & $a b$ & 12.66 & $7.20 ; 17.84$ \\
\hline & $\mathrm{m} 2$ & c & 20.93 & $20.06 ; 21.80$ & c & 19.78 & $18.25 ; 21.30$ & c & 20.55 & $18.34 ; 22.77$ \\
\hline & & $c^{\prime}$ & 20.73 & $19.38 ; 22.09$ & $c^{\prime}$ & 21.07 & $18.70 ; 23.44$ & $c^{\prime}$ & 19.84 & $16.39 ; 23.29$ \\
\hline & & $a b$ & 0.20 & $-0.86 ; 1.24$ & $a b$ & -1.29 & $-3.12 ; 0.44$ & $a b$ & 0.72 & $-1.97 ; 3.43$ \\
\hline & $\mathrm{m} 3$ & c & 20.93 & $20.06 ; 21.80$ & c & 19.78 & $18.25 ; 21.30$ & c & 20.55 & $18.34 ; 22.77$ \\
\hline & & $c^{\prime}$ & 21.18 & $20.18 ; 22.19$ & $c^{\prime}$ & 20.51 & $18.75 ; 22.26$ & $c^{\prime}$ & 21.10 & $18.54 ; 23.65$ \\
\hline & & $a b$ & -0.26 & $-0.73 ; 0.23$ & $a b$ & -0.73 & $-1.72 ; 0.20$ & $a b$ & -0.54 & $-1.89 ; 0.81$ \\
\hline$x 2$ & $\mathrm{~m} 1$ & c & 17.05 & $16.14 ; 17.96$ & c & 16.12 & $14.69 ; 17.56$ & c & 16.23 & $14.18 ; 18.28$ \\
\hline & & $c^{\prime}$ & 3.01 & $1.76 ; 4.26$ & $c^{\prime}$ & 3.45 & $1.07 ; 5.83$ & $c^{\prime}$ & 2.13 & $-1.38 ; 5.65$ \\
\hline & & $a b$ & 14.04 & $12.93 ; 15.25$ & $a b$ & 12.68 & $10.63 ; 14.66$ & $a b$ & 14.09 & $11.16 ; 17.13$ \\
\hline & $\mathrm{m} 2$ & c & 17.05 & $16.14 ; 17.96$ & c & 16.12 & $14.69 ; 17.56$ & c & 16.23 & $14.18 ; 18.28$ \\
\hline & & $c^{\prime}$ & 13.43 & $12.23 ; 14.63$ & $c^{\prime}$ & 13.60 & $11.67 ; 15.54$ & $c^{\prime}$ & 11.92 & $9.16 ; 14.68$ \\
\hline & & $a b$ & 3.62 & $2.81 ; 4.46$ & $a b$ & 2.52 & $1.24 ; 3.84$ & $a b$ & 4.31 & $2.36 ; 6.14$ \\
\hline & m3 & c & 17.05 & $16.14 ; 17.96$ & c & 16.12 & $14.69 ; 17.56$ & c & 16.23 & $14.18 ; 18.28$ \\
\hline & & $c^{\prime}$ & 16.26 & $15.24 ; 17.27$ & $c^{\prime}$ & 15.74 & $14.14 ; 17.34$ & $c^{\prime}$ & 15.54 & $13.25 ; 17.82$ \\
\hline & & $a b$ & 0.79 & $0.36 ; 1.27$ & $a b$ & 0.38 & $-0.40 ; 1.13$ & $a b$ & 0.69 & $-0.34 ; 1.75$ \\
\hline$x 3$ & $\mathrm{~m} 1$ & c & 8.58 & $7.74 ; 9.43$ & c & 8.26 & $7.12 ; 9.40$ & c & 7.65 & $6.09 ; 9.22$ \\
\hline & & $c^{\prime}$ & 0.58 & $-0.05 ; 1.20$ & $c^{\prime}$ & 0.92 & $-0.27 ; 2.10$ & $c^{\prime}$ & -0.42 & $-2.17 ; 1.32$ \\
\hline & & $a b$ & 8.00 & $7.30 ; 8.73$ & $a b$ & 7.35 & $6.46 ; 8.31$ & $a b$ & 8.08 & $6.90 ; 9.40$ \\
\hline & $\mathrm{m} 2$ & c & 8.58 & $7.74 ; 9.43$ & c & 8.26 & $7.12 ; 9.40$ & c & 7.65 & $6.09 ; 9.22$ \\
\hline & & $c^{\prime}$ & 4.66 & $3.84 ; 5.49$ & $c^{\prime}$ & 4.93 & $3.70 ; 6.17$ & $c^{\prime}$ & 3.45 & $1.74 ; 5.16$ \\
\hline & & $a b$ & 3.92 & $3.33 ; 4.54$ & $a b$ & 3.33 & $2.65 ; 4.06$ & $a b$ & 4.21 & $3.32 ; 5.19$ \\
\hline & m3 & c & 8.58 & $7.74 ; 9.43$ & c & 8.26 & $7.12 ; 9.40$ & c & 7.65 & $6.09 ; 9.22$ \\
\hline & & $c^{\prime}$ & 7.20 & $6.33 ; 8.08$ & $c^{\prime}$ & 7.17 & $5.97 ; 8.38$ & $c^{\prime}$ & 6.30 & $4.64 ; 7.95$ \\
\hline & & $a b$ & 1.38 & $1.03 ; 1.80$ & $a b$ & 1.09 & $0.64 ; 1.54$ & $a b$ & 1.36 & $0.76 ; 2.06$ \\
\hline
\end{tabular}


The magnitude of the mediated effect is dependent on the magnitude of the paths a and $b$, which are shown in Table 2.5.3. The regression coefficients for path a varied between $\beta=3.07(95 \% \mathrm{Cl} 3.00-3.16)$ and $\beta=1.20(95 \% \mathrm{Cl} 1.06-1.33)$, but were all statistically significant. The regression coefficient of path $b$ was $\beta=4.93(95 \% \mathrm{Cl}$ 4.32-5.55) for $m 1$ in the association $x 1-y 1$, and close to the true $\beta=5$. However, the regression coefficients for path $\mathrm{b}$ were considerably attenuated when mediators $\mathrm{m} 2$ or m3 were used, particularly when adjusted for proximal determinants $x 1$ and $x 2$. Consequently, some of the associations between the mediator and the outcome were no longer statistically significant.

Table 2.5.3 Influence of random error in determinant, mediator and outcome on paths $a$ and $b$ of mediation analysis.

Associations of $X$ with $M($ path a $\S)$

\begin{tabular}{|c|c|c|c|c|c|c|}
\hline \multicolumn{3}{|c|}{ m1 } & \multicolumn{2}{|l|}{$\mathrm{m} 2$} & \multicolumn{2}{|l|}{ m3 } \\
\hline & $\beta$ & $95 \% \mathrm{Cl}$ & $\beta$ & $95 \% \mathrm{Cl}$ & $\beta$ & $95 \% \mathrm{Cl}$ \\
\hline $\mathrm{x1}$ & 3.07 & $3.00 ; 3.16$ & 2.94 & $2.79 ; 3.10$ & 2.82 & $2.52 ; 3.13$ \\
\hline$x 2$ & 2.47 & $2.36 ; 2.57$ & 2.32 & $2.17 ; 2.48$ & 2.24 & $1.96 ; 2.53$ \\
\hline $\mathrm{x} 3$ & 1.26 & $1.15 ; 1.36$ & 1.20 & $1.06 ; 1.33$ & 1.29 & $1.08 ; 1.50$ \\
\hline
\end{tabular}

Associations of $\mathrm{M}$ with $\mathrm{Y}($ path $\mathrm{b} \S)$

\begin{tabular}{|c|c|c|c|c|c|c|c|}
\hline & & y1 & & y2 & & y3 & \\
\hline & & $\beta$ & $95 \% \mathrm{Cl}$ & $\beta$ & $95 \% \mathrm{Cl}$ & $\beta$ & $95 \% \mathrm{Cl}$ \\
\hline $\mathrm{m} 1$ & crude & 6.53 & $6.29 ; 6.77$ & 6.10 & $5.65 ; 6.55$ & 6.31 & $5.65 ; 6.97$ \\
\hline & $+x 1$ & 4.93 & $4.32 ; 5.55$ & 4.18 & $3.00 ; 5.35$ & 4.12 & $2.39 ; 5.84$ \\
\hline & $+x 2$ & 5.69 & $5.27 ; 6.11$ & 5.14 & $4.34 ; 5.94$ & 5.71 & $4.53 ; 6.90$ \\
\hline & $+x 3$ & 6.37 & $6.08 ; 6.67$ & 5.85 & $5.29 ; 6.4$ & 6.42 & $5.61 ; 7.25$ \\
\hline $\mathrm{m} 2$ & crude & 4.21 & $3.89 ; 4.52$ & 3.77 & $3.31 ; 4.22$ & 4.20 & $3.59 ; 4.82$ \\
\hline & $+x 1$ & 0.07 & $-0.29 ; 0.42$ & -0.44 & $-1.06 ; 0.18$ & 0.24 & $-0.66 ; 1.14$ \\
\hline & $+x 2$ & 1.56 & $1.21 ; 1.91$ & 1.09 & $0.52 ; 1.65$ & 1.85 & $1.05 ; 2.66$ \\
\hline & $+x 3$ & 4.66 & $3.84 ; 5.49$ & 2.79 & $2.29 ; 3.29$ & 3.52 & $2.82 ; 4.21$ \\
\hline m3 & crude & 1.79 & $1.53 ; 2.04$ & 1.56 & $1.23 ; 1.89$ & 1.68 & $1.24 ; 2.12$ \\
\hline & $+x 1$ & -0.09 & $-0.27 ; 0.09$ & -0.26 & $-0.57 ; 0.05$ & -0.19 & $-0.65 ; 0.26$ \\
\hline & $+x 2$ & 0.35 & $0.15 ; 0.55$ & 0.17 & $-0.15 ; 0.49$ & 0.31 & $-0.15 ; 0.76$ \\
\hline & $+x 3$ & 1.07 & $0.83 ; 1.32$ & 0.85 & $0.51 ; 1.18$ & 1.05 & $0.59 ; 1.51$ \\
\hline
\end{tabular}

$\S$ See also Figure 2.5.1

This simulation study of a fictional causal mechanism shows the influence of random error on all paths of mediation analysis. Although it seems obvious that random error in the mediator attenuates the size of the mediated effect, bootstrapping was still able to detect a significant mediated effect with the most imprecise combination of variables (i.e. x3-m3-y3). However, when a mediator was combined with a relatively more precise proximal determinant, mediated effects were considerably attenuated and failed to reach statistical significance. Such results should not simply be interpreted as a genuine lack of mediation, since the (causal) associations 
are in fact true in this fictional scenario. Instead, the lack of (statistically significant) mediation is due to random error in the mediator.

In addition, the imprecise variables $\mathrm{m} 2$ and $\mathrm{m} 3$ were significantly associated with $Y$ in univariate analysis, but not after adjustment for the more precise variables $\mathrm{x} 1$ or $\mathrm{x} 2$. From a mediation analysis point of view, the adjustment for a proximal causal factor is warranted in the evaluation of a causal relationship between $M$ and $Y$ (i.e. path b) $(1,3,6,15)$. The observed attenuation after adjustment in our simulation study (Table 2.5.3), however, must not be interpreted as a lack of (causal) association between mediator and outcome due to confounding, but as a consequence of adding a proximal causal factor that explains a considerable amount of variance in $\mathrm{Y}$ in linear regression analysis.

This fictional example allowed us to evaluate the influence of random error and to compare the results with the "true" associations. Obviously, in real epidemiological research we do not know the true associations and true causal mechanisms, and the distinction between attenuation due to random error in a mediator (i.e. an obscured mediated effect) and attenuation due to confounding (i.e. a true lack of an independent association between $M$ and $Y$, and hence lack of a mediated effect by $M$ ) may be difficult to make, particularly in cross-sectional studies. Therefore, it seems appropriate, at least, to report crude and adjusted associations, and to address both possibilities in the discussion and interpretation of research findings. Causal diagrams or direct acyclic graphs (DAGs) are an excellent way to present one's prior causal assumptions or beliefs based on the literature $(1,16)$.

\section{A practical application in metabolic research}

Serum complement factor 3 (C3) is a novel risk factor in obesity-related metabolic and cardiovascular diseases. It has been associated with incident type 2 diabetes mellitus, myocardial infarction, and hypertension (17-20). Several measures of body fat are associated with systemic C3 concentrations (21-23), and weight loss decreases and weight gain increases C3 levels (24). Although the association of body fat with C3 is clear, the mechanisms that underlie higher levels of serum C3 in obesity are still unknown. However, the low-grade inflammation accompanying obesity provides a potential pathway, since several pro-inflammatory cytokines such as tumour necrosis factor- $\alpha$ (TNF- $\alpha$ ), interleukin-1 (IL-1), interleukin-6 (IL-6) and interferon- $\gamma$ (IFN- $\gamma$ ) have been shown to increase complement factor expression in many cell types in vitro (25-27). By using comprehensive mediation analyses, we have shown that (an average score of) inflammatory cytokines significantly explained about $25 \%$ of fat-related differences in serum C3 in an epidemiological study of 532 subjects with increased cardiometabolic risk (9). Background information on C3 and the original 
article are in Chapter $\mathbf{4 . 2}$ of this thesis. Here, we will only discuss the influence of random error on the results of the mediation analyses.

For this purpose, we will consider the associations of two adiposity measures, namely waist circumference (WC) and body mass index (BMI), with serum C3 levels. As mediators, we present high-sensitivity C-reactive protein (hs-CRP), soluble inter-cellular adhesion molecule-1 (sICAM1) and interleukin-6 (IL-6) as markers of systemic low-grade inflammation. The interassay coefficient of variation, which is an indication of analytical measurement error, was $1.9 \%, 6.7 \%$ and $7.7 \%$ for hs-CRP, SICAM-1, and IL-6, respectively. All mediators had a skewed distribution and were In transformed prior to further analyses. Adiposity measurements, inflammatory markers and serum C3 were all standardized to enable comparison of the strengths of several associations.

The mediating role of inflammatory markers $(M)$ in the associations between estimates of body fat $(X)$ and $C 3(Y)$ was examined with the use of simple mediation models, as illustrated in Figure 2.5.2 (2). Linear regression analyses were used to calculate $\beta s$ and $95 \% \mathrm{Cls}$ of paths $\mathrm{a}, \mathrm{b}, \mathrm{c}$ and $\mathrm{c}^{\prime}$ in Figure 2.5.2. The magnitude of the mediated 'effects' by inflammatory markers (products $a b)$ is presented by $\beta$ s with respective (bias-corrected) $95 \% \mathrm{Cls}$ estimated by bootstrapping (5000 samples) (2). In agreement with the original article, all analyses were adjusted for age, sex, glucose metabolism status, prior cardiovascular disease, estimated glomerular filtration rate, smoking status, alcohol consumption and medication (covariates) (9). Analyses were performed using the Statistical Package for Social Sciences for Windows, version 17.0 (SPSS Inc., Chicago, IL, USA).

\section{Results}

Table 2.5.4 shows that both WC $(\beta=0.383 ; 95 \% \mathrm{Cl} 0.302-0.464)$ and $\mathrm{BMI}(\beta=0.320 ; 95 \% \mathrm{Cl}$ 0.243-0.398) were associated with serum C3 levels after adjustment for several covariates (paths $\mathrm{c}$ ). Mediation by inflammatory markers with increasing random error is shown by the ab paths in Table 2.5.4. The magnitude of the mediation in absolute values was $\beta=0.110(95 \% \mathrm{Cl} 0.076-0.150)$ for hs-CRP, $\beta=0.038(95 \% \mathrm{Cl} 0.018-0.070)$ for sICAM-1, and $\beta=0.015$ ( $95 \% \mathrm{Cl}-0.006$ to 0.039 ) for IL-6. Similar mediated effects were observed in the associations of BMI with C3. Overall, with increasing levels of random error in the mediator, the magnitude of the mediated effects decreased, leading to statistically non-significant results for IL-6. 

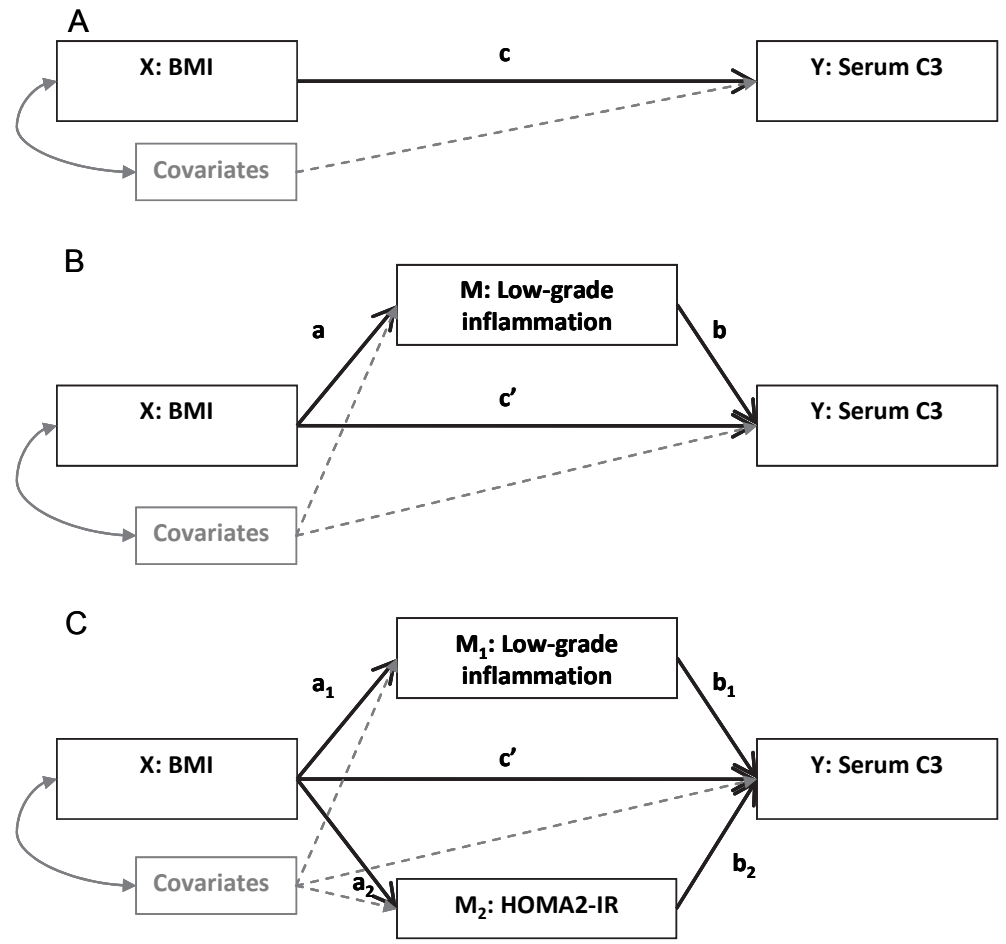

Figure 2.5.2 Causal diagrams for mediation analysis in the evaluation of low-grade inflammation as a mediator in the association between body fat and serum C3.

Panel A shows the association between an adiposity measure, e.g. body mass index (BMI), and serum C3 (dependent variable), adjusted for several covariates. Path $c$ is equal to the standardized linear regression coefficient $(\beta)$, and represents the overall association of an adiposity measurement with serum C3. Panel B illustrates how the role of low-grade inflammation (represented by three biomarkers in this paper) is assessed in simple mediation analysis. The product of regression coefficients $\mathrm{a}$ and $\mathrm{b}(\mathrm{ab})$ quantifies the indirect or mediated 'effect' by low-grade inflammation in the relation between an adiposity measure and serum C3. The part of the association not explained by low-grade inflammation is the direct 'effect' of the adiposity measurement on serum $\mathrm{C} 3$ and is represented by path $\mathrm{c}^{\prime}$. Panel $\mathrm{C}$ shows how the associations between mediators and serum $C_{3}$ independent of one another and of $B M I$ (paths $b_{1}$ and $b_{2}$ ) are assessed simultaneously in one regression model. Therefore, the independent mediated 'effect' by low-grade inflammation $\left(\mathrm{M}_{1}\right)$ in the relation between $\mathrm{BMI}$ and serum $\mathrm{C} 3$ is given by product $a_{1} b_{1}$, and the independent mediated 'effect' by HOMA2-IR $\left(M_{2}\right)$ is $a_{2} b_{2}$. The part of the association not explained by both mediating paths is the direct 'effect' $(\mathrm{c}$ ') of BMI on serum C3. 
Table 2.5.4 Mediation by inflammatory markers in the associations of waist circumference and body mass index with serum C3 levels.

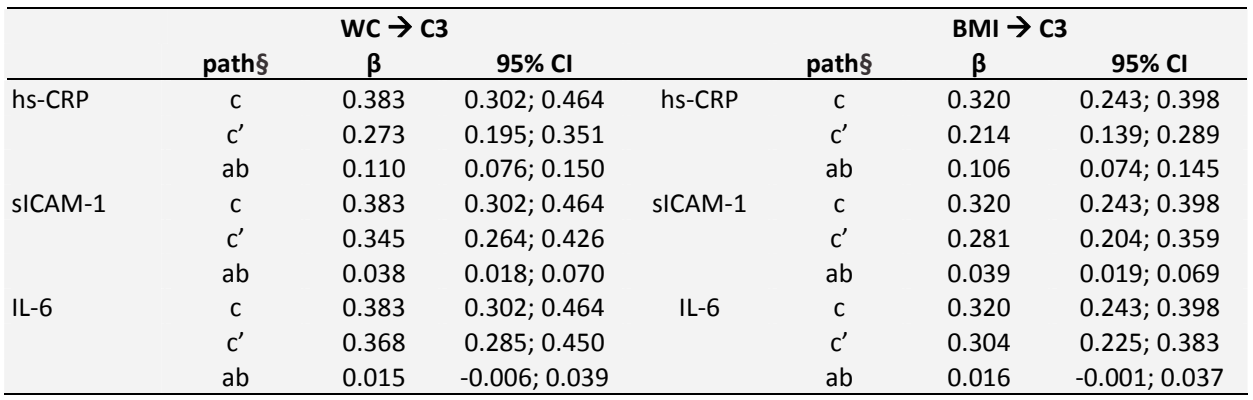

Paths $\mathrm{c}$ represent the overall associations between adiposity measures and C3 in standardized regression coefficients ( $\beta$ ), which reflect the change in C3 (in SD) per SD increase in the adiposity measurements. Path c' represents the direct association between adiposity measure and C3 that is not explained by the mediator considered; paths ab represent the portion of $c$ that is explained by the inflammatory marker. BMI: body mass index; C3: complement factor 3; hs-CRP: high sensitivity C-reactive protein; IL-6: interleukin-6; sICAM-1: soluble inter-cellular adhesion molecule-1; WC: waist circumference. §See also Figure 2.5.2.

Analogous to our simulation study, the decrease in the size of the mediated effect by increasing random error was mainly due to smaller effect sizes of path $b$ (i.e. the associations between inflammatory markers and C3 adjusted for adiposity measures), which can be seen in Table 2.5.5. Adjustment for the proximal causal factor adiposity (WC or BMI) attenuated all associations of inflammatory markers with C3 to some extent, but the attenuation is most striking for IL-6. The associations of adiposity measures with inflammatory markers (path a) were more or less comparable between the markers.

Table 2.5.5 Paths a and $\mathrm{b}$ of mediation analysis in associations between adiposity and C3.

Associations of adiposity measures with inflammatory markers (path a §)

\begin{tabular}{lcccccc}
\hline & \multicolumn{2}{c}{ hs-CRP } & \multicolumn{2}{c}{ sICAM-1 } & \multicolumn{2}{c}{ IL6 } \\
& $\boldsymbol{\beta}$ & $\mathbf{9 5 \%}$ Cl & $\mathbf{B}$ & $\mathbf{9 5 \%} \mathbf{~ C l}$ & $\boldsymbol{\beta}$ & $\mathbf{9 5 \%} \mathbf{~ C l}$ \\
\hline WC & 0.315 & $0.227 ; 0.404$ & 0.227 & $0.138 ; 0.317$ & 0.213 & $0.122 ; 0.303$ \\
BMI & 0.292 & $0.208 ; 0.376$ & 0.209 & $0.123 ; 0.295$ & 0.198 & $0.113 ; 0.283$ \\
\hline
\end{tabular}

Associations of inflammatory markers with serum C3 (path b §)

\begin{tabular}{lcccccc}
\hline & \multicolumn{2}{c}{ hs-CRP } & \multicolumn{2}{c}{ sICAM-1 } & \multicolumn{2}{c}{ IL6 } \\
& $\boldsymbol{\beta}$ & $\mathbf{9 5 \%}$ Cl & $\mathbf{B}$ & $\mathbf{9 5 \%}$ Cl & $\boldsymbol{\beta}$ & $\mathbf{9 5 \% ~ C l}$ \\
\hline crude & 0.425 & $0.352 ; 0.497$ & 0.243 & $0.165 ; 0.322$ & 0.141 & $0.060 ; 0.222$ \\
+WC & 0.350 & $0.277 ; 0.423$ & 0.180 & $0.104 ; 0.255$ & 0.067 & $-0.010 ; 0.145$ \\
+BMI & 0.364 & $0.290 ; 0.438$ & 0.186 & $0.109 ; 0.263$ & 0.081 & $0.003 ; 0.160$ \\
\hline
\end{tabular}

Paths a represent the associations between adiposity measures and inflammatory markers in standardized regression coefficients $(\beta)$, which reflect the change in the inflammatory marker (in SD) per SD increase in the adiposity measurements; paths b reflect the change in serum C3 (in SD) per SD increase in inflammatory markers, adjusted for WC and BMI, respectively. BMI: body mass index; C3: complement factor 3; hs-CRP: high sensitivity C-reactive protein; IL-6: interleukin-6; sICAM-1: soluble inter-cellular adhesion molecule-1; WC: waist circumference. §See also Figure 2.5.2. 
If only IL- 6 had been considered as a mediator of adiposity-related differences in serum $\mathrm{C} 3$, the conclusion of the mediation analysis would have been that there is no significant and/or relevant mediation by IL-6, and that IL- 6 is not (independently) associated with serum C3 levels. This would then have led to the erroneous conclusion that inflammation does not induce C3 production or secretion in obesity. However, when more inflammatory markers are considered, with less analytical measurement error, the conclusion is that, overall, inflammatory cytokines explain $25 \%$ of adiposityrelated changes in C3, and likely have a pathophysiological role in elevating C3 levels, in agreement with animal studies and human in vitro studies $(26,27)$.

As an analogous example, individual studies of inflammatory markers in relation to hypertension or type 2 diabetes mellitus have shown that after adjustment for proximal factor BMI, CRP was significantly associated with hypertension and diabetes, but IL- 6 was not $(28,29)$. Although the authors did not report analytical error, they acknowledge that IL-6 has a shorter plasma half-life, and its release is susceptible to diurnal variation, increasing its random error and biasing the effect sizes towards the null. These examples illustrate that using only one biomarker (e.g. IL-6) may not be sufficient to investigate pathophysiological processes, particularly when that marker is influenced by random error. The simultaneous use of multiple markers representing one construct (in this case low-grade inflammation), possibly in an averaged score, may avoid these problems, but is not yet standard practice in epidemiology.

\section{Extension to multiple mediation analysis}

The theory and analyses above are confined to simple mediation models, in which only one mediator is considered simultaneously. In practice however, there are often several different pathophysiological pathways, defined by several intermediate variables, hypothesized through which a determinant may be associated with an outcome. The independent, specific contribution of each mediator may then be estimated simultaneously in one multiple mediation model (Figure 2.5.2) (2,4). Extending the practical example of simple mediation described above, we considered both low-grade inflammation (defined by the inflammatory cytokines) and insulin resistance (defined by homeostasis model assessment insulin resistance; HOMA2-IR) as mediators in the association between obesity and $C 3$ in the original paper (9). Table 2.5.6 shows that HOMA2-IR was also a significant mediator in all models, and the same pattern of attenuation of mediated effects can be observed for the inflammatory cytokines in these multiple mediation models, as compared with the simple mediation models. The magnitude of the mediation in absolute values was $\beta=0.100$ (95\% Cl 0.069-0.138) for hs-CRP, $\beta=0.024$ (95\%Cl 0.007-0.049) for sICAM-1, and $\beta=0.011$ $(95 \% \mathrm{Cl}-0.007-0.034)$ for IL-6 in associations between waist circumference and C3. Similar mediated effects were observed in the associations of BMI with C3. Analogous 
to the simple mediation models, the magnitude of the mediated effects decreased with increasing levels of random error in the mediator, leading to statistically non-significant results for IL-6.

Table 2.5.6 Multiple mediation by inflammatory markers and insulin resistance in the associations of waist circumference and body mass index with serum C3 levels.

\begin{tabular}{|c|c|c|c|c|c|c|c|}
\hline & & & $7 \mathrm{C3}$ & & & BMI & C3 \\
\hline & path§ & $\beta$ & $95 \% \mathrm{Cl}$ & & path§ & $\beta$ & $95 \% \mathrm{Cl}$ \\
\hline & c & 0.383 & $0.302 ; 0.464$ & & c & 0.320 & $0.243 ; 0.398$ \\
\hline & $c^{\prime}$ & 0.098 & $0.012 ; 0.184$ & & $c^{\prime}$ & 0.043 & $-0.037 ; 0.123$ \\
\hline hs-CRP & $a_{1} b_{1}$ & 0.100 & $0.069 ; 0.138$ & hs-CRP & $a_{1} b_{1}$ & 0.095 & $0.065 ; 0.131$ \\
\hline HOMA2-IR & $a_{2} b_{2}$ & 0.185 & $0.134 ; 0.240$ & HOMA2-IR & $a_{2} b_{2}$ & 0.182 & $0.133 ; 0.235$ \\
\hline & c & 0.383 & $0.302 ; 0.464$ & & c & 0.320 & $0.243 ; 0.398$ \\
\hline & $c^{\prime}$ & 0.169 & $0.079 ; 0.259$ & & $c^{\prime}$ & 0.107 & $0.023 ; 0.192$ \\
\hline sICAM-1 & $a_{1} b_{1}$ & 0.024 & $0.007 ; 0.049$ & sICAM-1 & $a_{1} b_{1}$ & 0.024 & $0.007 ; 0.048$ \\
\hline HOMA2-IR & $a_{2} b_{2}$ & 0.190 & $0.138 ; 0.249$ & HOMA2-IR & $a_{2} b_{2}$ & 0.189 & $0.140 ; 0.246$ \\
\hline & c & 0.383 & $0.302 ; 0.464$ & & c & 0.320 & $0.243 ; 0.398$ \\
\hline & $c^{\prime}$ & 0.166 & $0.075 ; 0.258$ & & $c^{\prime}$ & 0.107 & $0.022 ; 0.192$ \\
\hline IL-6 & $a_{1} b_{1}$ & 0.011 & $-0.007 ; 0.034$ & IL-6 & $a_{1} b_{1}$ & 0.011 & $-0.005 ; 0.031$ \\
\hline HOMA2-IR & $a_{2} b_{2}$ & 0.206 & $0.153 ; 0.270$ & HOMA2-IR & $a_{2} b_{2}$ & 0.203 & $0.151 ; 0.260$ \\
\hline
\end{tabular}

Paths $\mathrm{c}$ represent the overall associations between adiposity measures and $\mathrm{C} 3$ in standardized regression coefficients ( $\beta$ ), which reflect the change in C3 (in SD) per SD increase in the adiposity measurements. Path c' represents the direct association between adiposity measure and $C 3$ that is not explained by the mediators considered; paths $a_{1} b_{1}$ represent the portion of $c$ that is explained by the inflammatory marker; paths $a_{2} b_{2}$ represent the portion of $\mathrm{c}$ that is explained by HOMA2-IR. BMI: body mass index; C3: complement factor 3; HOMA2-IR: homeostasis model assessment insulin resistance; hs-CRP: high sensitivity C-reactive protein; IL-6: interleukin-6; sICAM-1: soluble inter-cellular adhesion molecule-1; WC: waist circumference. §See also Figure 2.5.2.

\section{Conclusions}

The use of mediation analysis in biomedical research presents a unique opportunity to integrate knowledge of several pathophysiological pathways into a comprehensive analysis. However, investigators should bear in mind that the statistical analyses are perfect in theory, but in practice, the results depend on the level of random error (determined by analytical measurement error and biological variability within persons of the measure under investigation) (14). We have shown both by a simulation study and a practical example that random error in a mediator variable may attenuate the association between mediator (M) and outcome (Y) after adjustment for a relatively precise proximal causal factor $(\mathrm{X})$, and hence obscure a real mediated effect. Random error in the outcome usually does not affect unstandardized effect sizes, but decreases 
precision of estimated regression coefficients, and decreases standardized coefficients or correlation coefficients.

These findings have two important implications for research in the biomedical field. First, one needs to be aware of the influence of random error. Substantial random error in the mediator relative to the proximal causal factor will, in case of mediation analysis, erroneously yield no significant and/or relevant mediated effect. Especially when mediation is strongly expected based on the literature, caution is warranted in interpreting the results. Second, when the association between a determinant and outcome is attenuated by adjustment for a covariate, it is important to have defined a priori whether this covariate is a proximal causal factor (i.e. prior to the determinant in the causal pathway) or a confounder. Adjustment for a proximal causal factor that is measured with more precision than the determinant may attenuate the association. However, this should not be interpreted as an absent (causal) relation between determinant and outcome, since the distinction with confounding cannot be made. Therefore, epidemiological investigators should discuss the findings of mediation analysis carefully from different points of view. 


\section{References}

1. Hernan MA, Hernandez-Diaz S, Werler MM, Mitchell AA: Causal knowledge as a prerequisite for confounding evaluation: an application to birth defects epidemiology. Am J Epidemiol 2002;155: 176-184

2. Preacher $\mathrm{KJ}$, Hayes AF: Asymptotic and resampling strategies for assessing and comparing indirect effects in multiple mediator models. Behav Res Methods 2008;40:879-891

3. MacKinnon DP, Krull JL, Lockwood CM: Equivalence of the mediation, confounding and suppression effect. Prev Sci 2000;1:173-181

4. Hayes AF: Beyond Baron and Kenny: statistical mediation analysis in the new millenium. Commun Monogr 2009;76:408-420

5. Mackinnon DP, Fairchild AJ: Current Directions in Mediation Analysis. Curr Dir Psychol Sci 2009;18:16

6. Shrout PE, Bolger N: Mediation in experimental and nonexperimental studies: new procedures and recommendations. Psychol Methods 2002;7:422-445

7. Preacher KJ, Hayes AF: SPSS and SAS procedures for estimating indirect effects in simple mediation models. Behav Res Methods Instrum Comput 2004;36:717-731

8. Lockwood CM, DeFrancesco CA, Elliot DL, Beresford SA, Toobert DJ: Mediation analyses: applications in nutrition research and reading the literature. J Am Diet Assoc 2010;110:753-762

9. Wlazlo N, van Greevenbroek MM, Ferreira I, Jansen EJ, Feskens EJ, van der Kallen CJ, Schalkwijk CG, Bravenboer B, Stehouwer CD: Low-grade inflammation and insulin resistance independently explain substantial parts of the association between body fat and serum C3: The CODAM study. Metabolism 2012;61:1787-1796

10. Wlazlo N, van Greevenbroek MM, Ferreira I, Jansen EH, Feskens EJ, van der Kallen CJ, Schalkwijk CG, Bravenboer B, Stehouwer CD: Activated complement factor 3 is associated with liver fat and liver enzymes: the CODAM study. Eur J Clin Invest 2013;43:679-688

11. Wlazlo N, van Greevenbroek MM, Ferreira I, Jansen EH, Feskens EJ, van der Kallen CJ, Schalkwijk CG, Bravenboer B, Stehouwer CD: Iron metabolism is associated with adipocyte insulin resistance and plasma adiponectin: the Cohort on Diabetes and Atherosclerosis Maastricht (CODAM) study. Diabetes Care 2013;36:309-315

12. MacMahon S, Peto R, Cutler J, Collins R, Sorlie P, Neaton J, Abbott R, Godwin J, Dyer A, Stamler J: Blood pressure, stroke, and coronary heart disease. Part 1, Prolonged differences in blood pressure: prospective observational studies corrected for the regression dilution bias. Lancet 1990;335:765-774

13. Bollen K: Structural equations with latent variables. Wiley-Interscience, 1989

14. Whitlock G, Clark T, Vander Hoorn S, Rodgers A, Jackson R, Norton R, MacMahon S: Random errors in the measurement of 10 cardiovascular risk factors. Eur J Epidemiol 2001;17:907-909

15. Schisterman EF, Cole SR, Platt RW: Overadjustment bias and unnecessary adjustment in epidemiologic studies. Epidemiology 2009;20:488-495

16. Greenland S: Randomization, statistics, and causal inference. Epidemiology 1990;1:421-429

17. Muscari A, Bozzoli C, Puddu GM, Sangiorgi Z, Dormi A, Rovinetti C, Descovich GC, Puddu P: Association of serum C3 levels with the risk of myocardial infarction. Am J Med 1995;98:357-364

18. Engstrom G, Hedblad B, Eriksson KF, Janzon L, Lindgarde F: Complement C3 is a risk factor for the development of diabetes: a population-based cohort study. Diabetes 2005;54:570-575

19. Engstrom G, Hedblad B, Berglund G, Janzon L, Lindgarde F: Plasma levels of complement C3 is associated with development of hypertension: a longitudinal cohort study. J Hum Hypertens 2007;21: 276-282

20. Onat A, Hergenc G, Can G, Kaya Z, Yuksel H: Serum complement C3: a determinant of cardiometabolic risk, additive to the metabolic syndrome, in middle-aged population. Metabolism 2010;59:628-634

21. Gabrielsson BG, Johansson JM, Lonn M, Jernas M, Olbers T, Peltonen M, Larsson I, Lonn L, Sjostrom L, Carlsson B, Carlsson LM: High expression of complement components in omental adipose tissue in obese men. Obes Res 2003;11:699-708

22. Warnberg J, Nova E, Moreno LA, Romeo J, Mesana MI, Ruiz JR, Ortega FB, Sjostrom M, Bueno M, Marcos A: Inflammatory proteins are related to total and abdominal adiposity in a healthy adolescent population: the AVENA Study. Am J Clin Nutr 2006;84:505-512 
23. Weyer C, Tataranni PA, Pratley RE: Insulin action and insulinemia are closely related to the fasting complement C3, but not acylation stimulating protein concentration. Diabetes Care 2000;23:779-785

24. Pomeroy C, Mitchell J, Eckert E, Raymond N, Crosby R, Dalmasso AP: Effect of body weight and caloric restriction on serum complement proteins, including Factor D/adipsin: studies in anorexia nervosa and obesity. Clin Exp Immunol 1997;108:507-515

25. Yudkin JS, Stehouwer CD, Emeis JJ, Coppack SW: C-reactive protein in healthy subjects: associations with obesity, insulin resistance, and endothelial dysfunction: a potential role for cytokines originating from adipose tissue? Arterioscler Thromb Vasc Biol 1999;19:972-978

26. Trayhurn P, Wood IS: Adipokines: inflammation and the pleiotropic role of white adipose tissue. Br J Nutr 2004;92:347-355

27. Volanakis JE: Transcriptional regulation of complement genes. Annu Rev Immunol 1995;13:277-305

28. Sesso HD, Wang L, Buring JE, Ridker PM, Gaziano JM: Comparison of interleukin-6 and C-reactive protein for the risk of developing hypertension in women. Hypertension 2007;49:304-310

29. Pradhan AD, Manson JE, Rifai N, Buring JE, Ridker PM: C-reactive protein, interleukin 6, and risk of developing type 2 diabetes mellitus. JAMA 2001;286:327-334 
Chapter

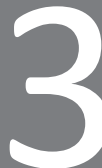

Chapter 3

Iron met

Iron metabolism

(a) 


\section{Chapter}

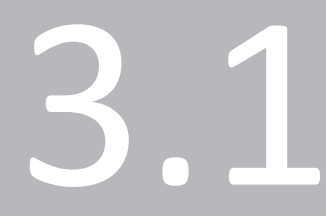

The role of iron metabolism in the metabolic syndrome and type 2 diabetes mellitus

Nick Wlazlo, Marleen MJ van Greevenbroek

Published in part as: Lipid metabolism: a role for iron? Curr Opin Lipidol;2012;23:258-259 
Chapter 3.1 


\section{Introduction into iron metabolism}

Iron is an essential nutrient for all human cell types. Iron is needed for the transport of oxygen (e.g. in haemoglobin) and electrons, and synthesis of DNA. It also represents a major catalyst for oxygenation, hydroxylation, and several other vital metabolic processes (see (1-5) for reviews). Iron exist in several oxidation states, of which the ferrous $\left(\mathrm{Fe}^{2+}\right)$ and ferric $\left(\mathrm{Fe}^{3+}\right)$ are the most common. The ferrous form particularly can react with oxygen and form highly reactive free radicals, which damage intracellular contents. Given this potentially dangerous high reactivity of free iron, iron is usually transported and stored bound to proteins. In plasma, most iron is present in the ferric $\left(\mathrm{Fe}^{3+}\right)$ form and bound to transferrin, which is usually saturated for $25-50 \%$. It is generally believed that, when the iron-binding capacity of transferrin is saturated (i.e. exceeds 50\%), iron emerges in serum as free ion, the so-called non-transferrinbound iron (NTBI), although recent data have also shown that plasma NTBI levels can already be detected when transferrin saturation is still within the normal range (6). NTBI may contribute to tissue iron overload and oxidative cellular damage, particularly in hepatocytes, cardiomyocytes, and pancreatic islet cells (7).

Transferrin-bound iron is delivered to cells in the bone marrow and liver through binding of transferrin with the transferrin receptor (TfR), of which soluble parts may also be detected in plasma (sTfR), particularly in iron deficiency. In addition, macrophages acquire iron via erythrophagocytosis of senescent erythrocytes. After internalisation of transferrin or erythrocytes by endocytosis, iron $\left(\mathrm{Fe}^{3+}\right)$ is released and reduced to its ferrous $\left(\mathrm{Fe}^{2+)}\right.$ form, and enters the intracellular pool, where it is either incorporated into haem (bone marrow) or stored as ferritin (liver, spleen, muscle). Ferritin is also present in serum, probably derived from hepatocytes, and is the most reliable marker of body iron stores $(1,2)$.

The human body iron content is usually $4-5 \mathrm{~g}$, of which the majority $(60 \%)$ is incorporated in haemoglobin in erythrocytes, and $15-30 \%$ is stored in cytoplasm of hepatocytes and in macrophages in the liver, spleen, bone marrow, and muscle (8). The amount of iron recycled daily by erythrophagocytosis of old erythrocytes by macrophages $(20-25 \mathrm{mg} / \mathrm{d})$ is usually sufficient to meet the demands for erythropoiesis in the bone marrow (Figure 3.1.1). In physiological conditions, 1-2 mg/d of iron is lost by exfoliation of cells from the gastro-intestinal tract and skin, and in women, by blood loss during menstruation. A normal human diet contains 13-18 mg iron, of which only 1-2 $\mathrm{mg}$ is absorbed to account for these losses. Haem iron (from meat) forms a small portion (7-12\%), and is well absorbed (for 25\%), but most dietary iron is non-haem iron (90\%) derived from vegetables, which is not easily absorbed (5\%) (1). After absorption into duodenal enterocytes and conversion to ferrous $\left(\mathrm{Fe}^{2+}\right)$ iron, intracellular iron is then processed either to ferritin (storage) or transported to the baso-lateral side of enterocytes into the circulation, where it is bound to transferrin in an oxidized form $\left(\mathrm{Fe}^{3+}\right)$. 


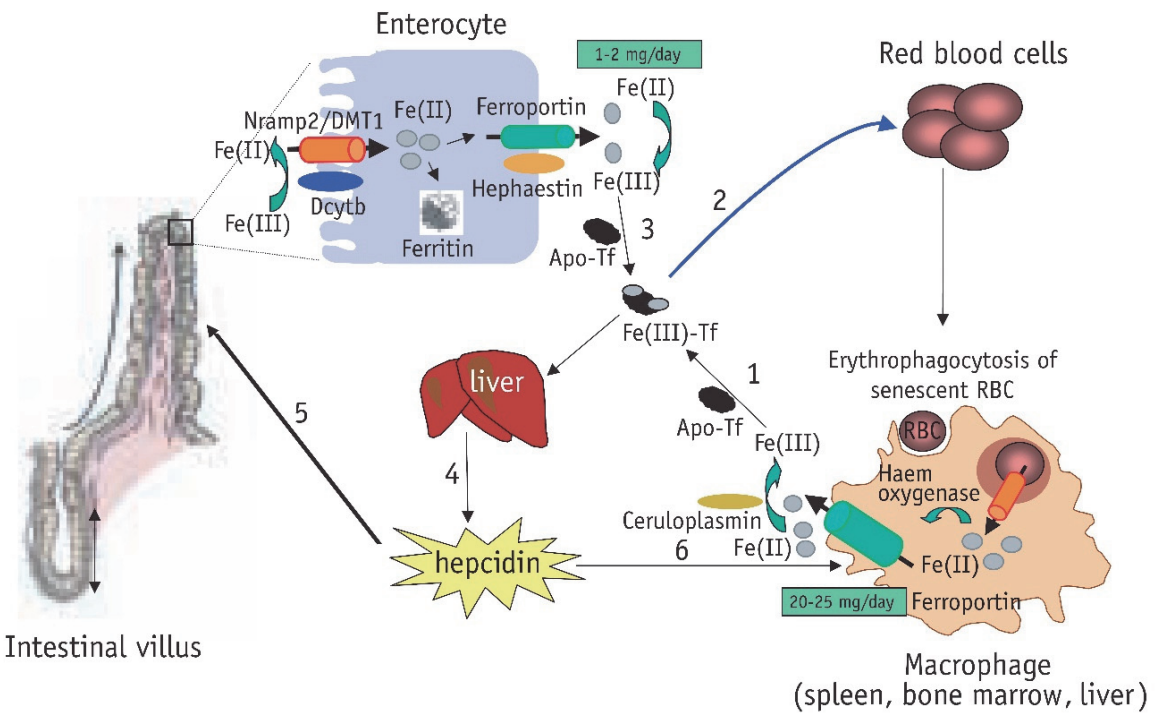

Figure 3.1.1 Iron homeostasis.

Iron required for erythropoiesis (20-25 mg daily) is provided by the destruction of senescent red blood cells by tissue macrophages (1). Iron released to the plasma by ferroportin is oxidized by ceruloplasmin and transported by transferrin to the erythroid precursors in the bone marrow (2). Intestinal iron absorption by duodenal enterocytes compensates for daily losses (3) (1-2 mg per day). Hepcidin, a cysteine-rich peptide synthesised by hepatocytes (4), negatively regulates iron export from enterocytes (5) and from macrophages (6) by binding to ferroportin and inducing its internalisation and degradation.

From The Handbook on Disorders of Erythropoiesis, Erythrocytes and Iron Metabolism (2).

The body and plasma iron balance is mainly determined by the amount of iron absorbed from the diet, as virtually no regulated mechanism for iron excretion is present. As iron stores and plasma iron levels increase, intestinal iron absorption is diminished by hepatic production of hepcidin, a protein that inhibits the release of iron by enterocytes and macrophages by degrading its transporter, ferroportin. In the absence of hepcidin, the ongoing intestinal absorption and release of iron by macrophages leads to parenchymal iron overload in several tissues, a mechanism believed to be key to hereditary haemochromatosis $(4,9)$. On the other hand, pro-inflammatory cytokines such as interleukin-6 (IL-6) have been shown to increase hepcidin production (10), leading to a retention of iron in enterocytes and macrophages and lower available serum iron, leading to the well-known anaemia of inflammation or anaemia of chronic disease commonly seen in patients with infections, malignancy, and inflammatory disorders $(5,11)$. 


\section{The role of iron in glucose metabolism}

Free iron is an important catalyst in the formation of highly reactive hydroxyl radicals (8), and intracellular reactive oxygen species have been shown to play a causal role in the induction of insulin resistance (12). Consequently, diabetes mellitus is very common in iron overload conditions such as hereditary haemochromatosis (9). Moreover, recent data have shown that modest iron overload (i.e. iron markers within the normal range) may also be involved in the pathogenesis of type 2 diabetes mellitus (T2DM) (13-18). Prospective studies have linked serum ferritin (13-15), as a surrogate of body iron stores, and dietary haem iron intake (16-18), (mainly derived from red meat), to incident T2DM.

Several cross-sectional studies have shown that serum ferritin is associated with T2DM and with insulin resistance (IR), either measured by homeostasis model assessment (HOMA-IR) or by hyperinsulinaemic euglycaemic clamp (19-25). On the other hand, serum ferritin and hepatic iron load (measured on MRI) have not been clearly associated with beta cell function $(19,25,26)$, indicating that the contribution of iron metabolism and iron metabolism-related factors in the pathophysiology of T2DM may be primarily related to the development of IR. Prospective data on the relation between markers of iron metabolism and IR are scarce. Only a single nested case-control study has shown that baseline ferritin and transferrin were associated with increases in insulin and new-onset hyperglycaemia over 3 years (27), and with a high HOMA-IR at 6 years (28). However, these analyses were not adjusted for regular risk factors for IR such as dietary energy intake, physical activity, smoking status, or a positive family history of T2DM. In addition, only HOMA-IR was used as a measure of IR.

It is currently unclear in which tissues iron may induce IR (e.g. muscle, liver, adipose tissue), since most studies evaluated only fasting glucose and/or insulin, HOMA-IR, or whole-body glucose disposal. These variables mainly reflect muscle IR (29), while they also correlate with hepatic IR. In agreement, in a mouse model of haemochromatosis, increased muscle iron load caused a shift from glucose oxidation to fatty acid oxidation in muscle, leading to substrate flux to the liver and increased hepatic glucose production (30). Moreover, increased hepatic iron content in mice, resulting from an iron-enriched diet, led to increased systemic IR (31). Furthermore, in vitro research has shown that iron-laden Hep2G (liver) cells had reduced expression of the insulin receptor and glucokinase genes, as well as reduced cellular insulin binding (32). In addition, hepatic iron content in humans has been associated with higher IR (26).

Interestingly, recent in vitro and animal studies have suggested that adipose tissue may also represent a target organ for iron. Incubation of rat adipocytes with iron decreased insulin-stimulated glucose transport and increased lipolysis in adipocytes $(33,34)$. In addition, an iron-enriched diet led to iron accumulation and IR in visceral adipose tissue in mice (31), whereas an iron-restricted diet led to lower free 
fatty acids and triglycerides (35). However, epidemiological data on the effect of iron on adipocyte insulin resistance in humans are not available, except for correlations of serum ferritin with plasma adiponectin levels $(14,36)$.

Altogether, there is considerable evidence that iron or iron metabolism-related factors may influence insulin signalling and glucose metabolism, and contribute to the pathogenesis of T2DM. Although several prospective studies have linked serum ferritin with incident T2DM (13-15), longitudinal data on the association of iron metabolism with IR are as yet scarce. In addition, most studies are limited to the use of ferritin only, and have not included other markers of iron metabolism mentioned above. Moreover, most epidemiological studies have only evaluated HOMA-IR as measure of IR, but it is still unclear in which organs iron may contribute to the development of IR. Given the data above, the liver and adipose tissue seem to represent interesting target organs for iron as well.

\section{The role of iron in lipid metabolism}

Iron metabolism has been linked to the development of glucose metabolism disorders as described above, but recent data have shown that iron metabolism may also influence lipid metabolism. Several large epidemiological studies in the general population have shown consistent associations of serum ferritin with higher triglycerides and lower high-density lipoprotein (HDL) cholesterol, i.e. the dyslipidaemia of the metabolic syndrome and T2DM $(20-22,24,25)$. The French DESIR study additionally showed that, during 6 years of follow-up, both serum ferritin and transferrin were positively associated with hypertriglyceridaemia and low HDL cholesterol (28). Moreover, in familial combined hyperlipidaemia and familial hypertriglyceridaemia, elevated ferritin levels were detected in $20 \%$ of patients, and serum ferritin showed a stronger correlation with serum triglycerides than adiposity measures, HOMA-IR, or liver enzymes (37). Finally, in healthy teenagers, decreasing quartiles of STfR/ferritin (indicating higher iron stores) were associated with higher triglycerides and with lower HDL-C (38). In summary, these findings suggest that iron, or iron-metabolism-related factors, may contribute to the dyslipidaemia observed in the metabolic syndrome.

The liver is a key organ in both iron and lipid metabolism, and both liver fat and impaired insulin-mediated suppression of hepatic triglyceride synthesis and release (i.e. hepatic IR regarding lipids) have been postulated to be major contributors to the high triglycerides / low HDL dyslipidaemia, as present in e.g. the metabolic syndrome and T2DM $(39,40)$. Therefore, the liver may represent the link between iron metabolism and dyslipidaemia. Indeed, the epidemiological studies described above also reported associations of ferritin, transferrin, and STfR/ferritin ratio with liver enzymes such as alanine aminotransferase (ALT) and gamma glutamyltransferase 
(GGT) $(25,28,37,38)$. Moreover, a recent study showed that higher hepatic iron stores (measured by magnetic resonance imaging) were associated with higher liver fat content (measured by magnetic resonance spectroscopy), and also with IR (estimated from OGTT) (26). Unfortunately, this study did not measure hepatic IR regarding triglyceride synthesis and release. In addition to the handling of lipids by the liver, the metabolism of triglycerides and free fatty acids in adipose tissue may also be influenced by iron-induced adipocyte IR as described above, and thus further contribute to the dyslipidaemic phenotype.

\section{Outline and aims of this chapter}

There is growing evidence that body iron stores and iron metabolism-related factors contribute to the development of disorders in glucose and lipid metabolism, such as T2DM and the metabolic syndrome. These effects of iron could be mediated through the induction of IR, resulting in impaired glucose tolerance and impaired insulinmediated suppression of free fatty acid and triglyceride output. Epidemiological data on this subject are largely limited to cross-sectional associations of serum ferritin with HOMA2-IR, with scarce longitudinal data available. Moreover, it is as yet unclear in which organs iron may contribute to the development of IR, but the liver and adipose tissue seem to represent important target organs for iron.

For these reasons, we further explored the relation of iron and iron metabolismrelated factors with IR in two epidemiological studies within the Cohort on Diabetes and Atherosclerosis Maastricht (CODAM) study, in which no less than 5 markers of iron metabolism were measured in the baseline evaluation (serum ferritin, transferrin, serum total iron, non-transferrin bound iron (NTBI), and transferrin saturation). First, we investigated cross-sectional associations of all these markers of iron metabolism with adipocyte IR and related traits (such as plasma adiponectin levels) (Chapter 3.2). In this study, we also investigated the potential role of oxidative stress in these associations. Second, we examined prospectively whether baseline markers of iron stores and iron metabolism were associated with (changes in) IR in muscle, liver, and adipocytes over a 7-year follow-up period (Chapter 3.3). In addition, we investigated associations of these markers with (progression of) glucose intolerance during 7-year follow-up. 


\section{References}

1. Crichton R, Danielson B, Geisser P: Iron Therapy With Special Emphasis on Intravenous Administration. Bremen, UNI-MED Verlag AG, 2008

2. Chapter 20. Iron Homeostasis. In The Handbook on Disorders of Erythropoiesis, Erythrocytes and Iron Metabolism, 2nd edition ed. Beaumont C, Béris P, Beuzard Y, Brugnara C, Eds. Genoa, Italy, Forum service editore, 2009

3. Hentze MW, Muckenthaler MU, Andrews NC: Balancing acts: molecular control of mammalian iron metabolism. Cell 2004;117:285-297

4. Andrews NC: Forging a field: the golden age of iron biology. Blood 2008;112:219-230

5. De Domenico I, McVey Ward D, Kaplan J: Regulation of iron acquisition and storage: consequences for iron-linked disorders. Nature reviews Molecular cell biology 2008;9:72-81

6. Lee DH, Liu DY, Jacobs DR, Jr., Shin HR, Song K, Lee IK, Kim B, Hider RC: Common presence of nontransferrin-bound iron among patients with type 2 diabetes. Diabetes Care 2006;29:1090-1095

7. Fleming RE, Ponka P: Iron overload in human disease. N Engl J Med 2012;366:348-359

8. Beard JL: Iron biology in immune function, muscle metabolism and neuronal functioning. J Nutr 2001;131:568S-579S; discussion 580S

9. Pietrangelo A: Hereditary hemochromatosis--a new look at an old disease. N Engl J Med 2004;350: 2383-2397

10. Nemeth E, Tuttle MS, Powelson J, Vaughn MB, Donovan A, Ward DM, Ganz T, Kaplan J: Hepcidin regulates cellular iron efflux by binding to ferroportin and inducing its internalization. Science 2004; 306:2090-2093

11. Means RT, Jr.: Hepcidin and anaemia. Blood Rev 2004;18:219-225

12. Houstis N, Rosen ED, Lander ES: Reactive oxygen species have a causal role in multiple forms of insulin resistance. Nature 2006;440:944-948

13. Jiang R, Manson JE, Meigs JB, Ma J, Rifai N, Hu FB: Body iron stores in relation to risk of type 2 diabetes in apparently healthy women. JAMA 2004;291:711-717

14. Forouhi NG, Harding AH, Allison M, Sandhu MS, Welch A, Luben R, Bingham S, Khaw KT, Wareham NJ: Elevated serum ferritin levels predict new-onset type 2 diabetes: results from the EPIC-Norfolk prospective study. Diabetologia 2007;50:949-956

15. Montonen J, Boeing H, Steffen A, Lehmann R, Fritsche A, Joost HG, Schulze MB, Pischon T: Body iron stores and risk of type 2 diabetes: results from the European Prospective Investigation into Cancer and Nutrition (EPIC)-Potsdam study. Diabetologia 2012;55:2613-2621

16. Jiang R, Ma J, Ascherio A, Stampfer MJ, Willett WC, Hu FB: Dietary iron intake and blood donations in relation to risk of type 2 diabetes in men: a prospective cohort study. Am J Clin Nutr 2004;79:70-75

17. InterAct C: Association between dietary meat consumption and incident type 2 diabetes: the EPICInterAct study. Diabetologia 2013;56:47-59

18. Lee DH, Folsom AR, Jacobs DR, Jr.: Dietary iron intake and Type 2 diabetes incidence in postmenopausal women: the lowa Women's Health Study. Diabetologia 2004;47:185-194

19. Haap M, Fritsche A, Mensing HJ, Haring HU, Stumvoll M: Association of high serum ferritin concentration with glucose intolerance and insulin resistance in healthy people. Ann Intern Med 2003;139:869-871

20. Jehn M, Clark JM, Guallar E: Serum ferritin and risk of the metabolic syndrome in U.S. adults. Diabetes Care 2004;27:2422-2428

21. Syrovatka P, Kraml P, Potockova J, Fialova L, Vejrazka M, Crkovska J, Andel M: Relationship between increased body iron stores, oxidative stress and insulin resistance in healthy men. Ann Nutr Metab 2009;54:268-274

22. Bozzini C, Girelli D, Olivieri O, Martinelli N, Bassi A, De Matteis G, Tenuti I, Lotto V, Friso S, Pizzolo F, Corrocher R: Prevalence of body iron excess in the metabolic syndrome. Diabetes Care 2005;28: 2061-2063

23. Chen J, Wildman RP, Hamm LL, Muntner P, Reynolds K, Whelton PK, He J: Association between inflammation and insulin resistance in U.S. nondiabetic adults: results from the Third National Health and Nutrition Examination Survey. Diabetes Care 2004;27:2960-2965 
24. Lee BK, Kim Y, Kim YI: Association of serum ferritin with metabolic syndrome and diabetes mellitus in the South Korean general population according to the Korean National Health and Nutrition Examination Survey 2008. Metabolism 2011;60:1416-1424

25. Kim CH, Kim HK, Bae SJ, Park JY, Lee KU: Association of elevated serum ferritin concentration with insulin resistance and impaired glucose metabolism in Korean men and women. Metabolism 2011;60: 414-420

26. Haap M, Machann J, von Friedeburg C, Schick F, Stefan N, Schwenzer NF, Fritsche A, Haring HU, Thamer C: Insulin sensitivity and liver fat: role of iron load. J Clin Endocrinol Metab 2011;96:E958-961

27. Fumeron F, Pean F, Driss F, Balkau B, Tichet J, Marre M, Grandchamp B: Ferritin and transferrin are both predictive of the onset of hyperglycemia in men and women over 3 years: the data from an epidemiological study on the Insulin Resistance Syndrome (DESIR) study. Diabetes Care 2006;29: 2090-2094

28. Vari IS, Balkau B, Kettaneh A, Andre P, Tichet J, Fumeron F, Caces E, Marre M, Grandchamp B, Ducimetiere $P$ : Ferritin and transferrin are associated with metabolic syndrome abnormalities and their change over time in a general population: Data from an Epidemiological Study on the Insulin Resistance Syndrome (DESIR). Diabetes Care 2007;30:1795-1801

29. Wallace TM, Levy JC, Matthews DR: Use and abuse of HOMA modeling. Diabetes Care 2004;27: 1487-1495

30. Huang J, Jones D, Luo B, Sanderson M, Soto J, Abel ED, Cooksey RC, McClain DA: Iron overload and diabetes risk: a shift from glucose to Fatty Acid oxidation and increased hepatic glucose production in a mouse model of hereditary hemochromatosis. Diabetes 2011;60:80-87

31. Dongiovanni P, Ruscica M, Rametta R, Recalcati S, Steffani L, Gatti S, Girelli D, Cairo G, Magni P, Fargion $S$, Valenti L: Dietary iron overload induces visceral adipose tissue insulin resistance. The American journal of pathology 2013;182:2254-2263

32. Fargion S, Dongiovanni P, Guzzo A, Colombo S, Valenti L, Fracanzani AL: Iron and insulin resistance. Aliment Pharmacol Ther 2005;22 Suppl 2:61-63

33. Rumberger JM, Peters T, Jr., Burrington C, Green A: Transferrin and iron contribute to the lipolytic effect of serum in isolated adipocytes. Diabetes 2004;53:2535-2541

34. Green A, Basile R, Rumberger JM: Transferrin and iron induce insulin resistance of glucose transport in adipocytes. Metabolism 2006;55:1042-1045

35. Cooksey RC, Jones D, Gabrielsen S, Huang J, Simcox JA, Luo B, Soesanto Y, Rienhoff H, Abel ED, McClain DA: Dietary iron restriction or iron chelation protects from diabetes and loss of beta-cell function in the obese (ob/ob lep-/-) mouse. Am J Physiol Endocrinol Metab 2010;298:E1236-1243

36. Ku BJ, Kim SY, Lee TY, Park KS: Serum ferritin is inversely correlated with serum adiponectin level: population-based cross-sectional study. Dis Markers 2009;27:303-310

37. Mateo-Gallego R, Calmarza P, Jarauta E, Burillo E, Cenarro A, Civeira F: Serum ferritin is a major determinant of lipid phenotype in familial combined hyperlipidemia and familial hypertriglyceridemia. Metabolism 2010;59:154-158

38. Aigner E, Hinz C, Steiner K, Rossmann B, Pfleger J, Hohla F, Steger B, Stadlmayr A, Patsch W, Datz C: Iron stores, liver transaminase levels and metabolic risk in healthy teenagers. Eur J Clin Invest 2010;40: 155-163

39. Malmstrom R, Packard CJ, Caslake M, Bedford D, Stewart P, Yki-Jarvinen H, Shepherd J, Taskinen MR: Defective regulation of triglyceride metabolism by insulin in the liver in NIDDM. Diabetologia 1997;40: 454-462

40. Fabbrini E, Magkos F, Mohammed BS, Pietka T, Abumrad NA, Patterson BW, Okunade A, Klein S: Intrahepatic fat, not visceral fat, is linked with metabolic complications of obesity. Proc Natl Acad Sci U S A 2009;106:15430-15435 


\section{Chapter}

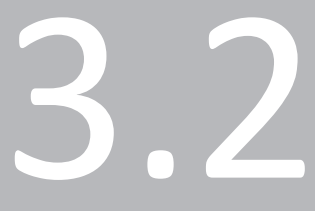

Iron metabolism is associated with adipocyte insulin resistance and plasma adiponectin

Nick Wlazlo, Marleen MJ van Greevenbroek, Isabel Ferreira, Eugene HJM Jansen, Edith JM Feskens, Carla JH van der Kallen, Casper G Schalkwijk, Bert Bravenboer, Coen DA Stehouwer 


\section{Abstract}

\section{Objective}

Adipocyte insulin resistance $(I R)$ is a key feature early in the pathogenesis of type 2 diabetes-mellitus (T2DM) and, although scarce, data in the literature suggest a direct role for iron and iron-metabolism-related factors in adipose tissue function and metabolism. Although serum ferritin and transferrin were shown to be associated with muscle insulin resistance (IR) and T2DM, little is known about the role of iron metabolism on adipose tissue. We therefore investigated whether markers of iron metabolism were associated with adipocyte IR and plasma adiponectin.

\section{Research design and methods}

Serum ferritin, transferrin, total iron, non-transferrin-bound iron (NTBI), transferrin saturation, and plasma adiponectin were determined in 492 individuals. Adipocyte IR was defined by the product of fasting insulin and non-esterified fatty acids (NEFAs). Using linear regression analyses, we investigated the difference in adipocyte IR or adiponectin (in \%) according to differences in iron metabolism markers.

\section{Results}

Serum ferritin ( $\beta=1.00 \%$ increase in adipocyte IR per $10 \mu \mathrm{g} / \mathrm{l} ; 95 \% \mathrm{Cl} 0.66-1.34$ ), transferrin $(\beta=4.18 \%$ per $0.1 \mathrm{~g} / \mathrm{l} ; 95 \% \mathrm{Cl} 2.88-5.50)$, total iron $(\beta=1.36 \%$ per $\mu \mathrm{mol} / \mathrm{l} ; 95 \% \mathrm{Cl} 0.61-2.12)$, and NTBI ( $\beta=5.14 \%$ per $\mu \mathrm{mol} / \mathrm{l} ; 95 \% \mathrm{Cl} 1.88-8.52)$ were associated with adipocyte IR after adjustment for several covariates, including inflammatory markers. All markers of iron metabolism were also associated with NEFAs (all $\mathrm{p}<0.01$ ). In addition, ferritin and transferrin were inversely associated with adiponectin (both $\mathrm{p}<0.01$ ).

\section{Conclusions}

The observed associations of several markers of iron metabolism with adipocyte IR and adiponectin suggest that iron and iron-metabolism-related factors may contribute to adipocyte IR early in the pathogenesis of T2DM. 


\section{Introduction}

Iron is an important catalyst in the formation of highly reactive hydroxyl radicals $(1,2)$, and intracellular reactive oxygen species have been shown to play an important causal role in the induction of insulin resistance (3). Consequently, massive iron overload, as present in hereditary haemochromatosis, frequently leads to diabetes mellitus (4). In addition, recent data have shown that modest iron overload (i.e. iron markers within the normal range) may also be involved in the pathogenesis of type 2 diabetes mellitus (T2DM) in general (5-7). Prospective studies have linked serum ferritin, the most reliable marker of body iron stores, and dietary haem iron intake to incident T2DM (5-7). Several cross-sectional and longitudinal studies have shown that serum ferritin is associated with muscle insulin resistance (IR), either measured by homeostasis model assessment (HOMA-IR) or by hyperinsulinemic euglycemic clamp (1,8-12), but not with beta cell function (8). These results suggest a contribution of iron to the pathogenesis of T2DM that is primarily related to the induction of insulin resistance.

Little is known, however, about the possible sites (e.g. muscle, liver, adipose tissue) where iron may induce insulin resistance, as most studies have only evaluated HOMA-IR or whole-body glucose disposal. Some studies have suggested that adipose tissue may be a primary target organ for metabolic effects of iron. Incubation of rat adipocytes with iron decreased insulin-stimulated glucose transport, and increased lipolysis in adipocytes $(13,14)$. In addition, an iron-enriched diet led to iron accumulation and insulin resistance in visceral adipose tissue in mice (15), whereas an iron-restricted diet led to lower free fatty acids and triglycerides (16). In one epidemiological study, the association between ferritin and incident T2DM was attenuated after adjustment for serum adiponectin (7), which suggests mediation by adipocyte insulin resistance (adipocyte IR). However, the effect of iron on adipose tissue is yet far from clear, and in vivo data on the effect of iron on adipocyte insulin resistance are not available, except for correlations of serum ferritin with adiponectin (17).

For this reason, we investigated the associations of several markers of iron metabolism with adipocyte IR and related traits in the Cohort on Diabetes and Atherosclerosis Maastricht (CODAM) study, which is a cohort study of subjects with (an increased risk of) T2DM and cardiovascular diseases (CVD) who underwent extensive metabolic characterisation. In a secondary analysis, we also investigated the potential role of oxidative stress in these associations. 


\section{Research design and methods}

\section{Subjects and study design}

CODAM participants were selected from a large cohort in the general population as described in detail elsewhere (18-20). Briefly, subjects were selected if they had: Caucasian ethnicity, $>40$ years of age, and at least one of the following inclusion criteria: a positive family history of T2DM (first degree), history of gestational diabetes, a BMI $\geq 25 \mathrm{~kg} / \mathrm{m}^{2}$, use of antihypertensive drugs, a postprandial glucose $\geq 6.0 \mathrm{mmol} / \mathrm{l}$ or glycosuria. In total, 574 individuals were included and extensively characterized with regard to their lifestyle, cardiovascular and metabolic profile during two visits to the University's metabolic research unit.

We performed the current cross-sectional analyses in the baseline evaluation of this cohort and excluded participants with insulin therapy $(n=13)$ or self-reported (history of) liver disease $(n=7)$. To exclude possible hereditary haemochromatosis, we excluded subjects with elevated serum ferritin ( $>150 \mu \mathrm{g} / \mathrm{l}$ for premenopausal women, $>400 \mu \mathrm{g} / \mathrm{I}$ for men and postmenopausal women) and elevated transferrin saturation ( $>45 \%)$ $(n=21)$ according to Dutch guidelines (21). Finally, subjects with missing data on iron markers $(n=9)$, glucose or insulin $(n=6)$, adiponectin $(n=7)$, or important covariates $(n=19)$ were also excluded. Hence, the present study reports on 492 subjects.

The study protocol was approved by the Medical Ethical Committee of the Maastricht University Medical Centre, and all subjects gave written informed consent.

\section{Markers of iron stores and iron metabolism}

Participants were asked to stop their lipid-lowering drugs 14 days before the visit, and to stop all other medication the day before the visit. After an overnight fast, venous blood samples were collected for assessment of several biomarkers, and an oral glucose tolerance test (OGTT) was performed. Serum was allowed to clot at room temperature for $45 \mathrm{~min}$, and after centrifugation at $3000 \mathrm{rpm}$ for $15 \mathrm{~min}$, serum aliquots were stored at $-20^{\circ} \mathrm{C}$, and plasma (EDTA) aliquots were stored at $-80^{\circ} \mathrm{C}$ until use. Samples were thawed only once prior to measurements.

Ferritin, transferrin, total iron, and unsaturated iron-binding capacity (UIBC) were determined in serum on a Hitachi 912 auto analyzer using assays \#1661400, \#1931628, $\# 1876996$, and \#1030600, respectively (Roche Diagnostics, Almere, The Netherlands). Non-transferrin-bound iron (NTBI) levels were measured in serum by a method using iron-sensitive fluorescence-labelled apotransferrin, as described in detail previously (22). All inter-assay coefficients of variation were $<5 \%$. Transferrin saturation was calculated as total serum iron/(total serum iron + UIBC). 


\section{Insulin resistance and adiponectin}

Plasma glucose levels were measured in NaF/KOx plasma with a hexokinase glucose- 6 phosphate dehydrogenase method (ABX Diagnostics). Insulin concentrations were determined in EDTA plasma using a two-sided immunoradiometric test using paired monoclonal antibodies (Medgenix Diagnostics). Non-esterified free fatty acids (NEFAs) were measured in EDTA plasma using an enzymatic calorimetric NEFA C method (Wako Diagnostics, Richmond, USA).

Adipocyte insulin resistance (adipocyte IR) was calculated as the product of fasting insulin and fasting NEFA concentrations, as described before (23-25). This index provides a valid marker of the sensitivity of adipocytes to the anti-lipolytic effect of insulin, since NEFA concentrations and fatty acid turnover in clamp studies are highly correlated, and insulin suppresses lipolysis already at low (i.e. fasting) concentrations $(10-20 \mathrm{mU} / \mathrm{I})(23,26)$. HOMA2-IR, which has been shown to correlate well with glucose disposal in clamp studies (27), was computed using the HOMA-calculator (http://www.dtu.ox.ac.uk /homacalculator/index.php). Total adiponectin was measured in EDTA plasma with a competitive ELISA kit (BioVendor Laboratory Medicine Inc, Brno, Czech Republic).

\section{Other covariates}

BMI was calculated as weight $(\mathrm{kg})$ divided by height in meters squared. Waist circumference was measured in $\mathrm{cm}$ with a tape centimetre at the level midway between the lateral lower rib margin and the anterior superior iliac spine. Smoking behaviour and family history of T2DM (first-degree relatives) were assessed by questionnaire. Dietary calorie intake and mean alcohol consumption were estimated by a validated food frequency questionnaire (20). Physical activity was derived from a validated short physical activity questionnaire (SQUASH) (28).

Malondialdehyde (MDA) was measured in EDTA with a reagent kit for HPLC analyses (Chromsystems Instruments and Chemicals, Munich, Germany) (Inter-assay CV 4-11\%), and total antioxidative status (TAS) was measured in serum with an enzymatic kit (Randox Diagnostics, Country Antrim, UK) (inter-assay CV 2.5\%). Serum interleukin-6 (IL-6), interleukin-8 (IL-8), tumour necrosis factor- $\alpha$ (TNF- $\alpha$ ), high sensitivity C-reactive protein (hs-CRP), serum amyloid A (SAA) and soluble inter-cellular adhesion molecule-1 (sICAM-1) were determined on a multi-array detection system based on electrochemiluminicence technology (SECTOR Imager 2400, Meso Scale Discovery, USA) (29). Creatinine levels were determined in EDTA plasma with a Jaffé diagnostic test (Roche Diagnostics), and glomerular filtration rate (eGFR) was estimated using the short Modification of Diet in Renal Disease (MDRD) equation (30). Glucose metabolism status (i.e. normal, impaired, or T2DM) was diagnosed according to the WHO 1999 criteria as previously described (18-20). Prior CVD was defined on the basis of (self-reported) 
history of CVD, signs of myocardial infarction or ischaemia on ECG, or an ankle-arm index $<0.9$ in either leg, as previously described in detail $(18,19)$.

\section{Statistical analysis}

Variables with a skewed distribution (i.e. ferritin, adipocyte IR, HOMA2-IR, adiponectin, all inflammatory markers) were In transformed prior to further analyses. Because several subjects had undetectable NTBI levels (i.e. $<0.01 \mu \mathrm{mol} / \mathrm{l}$; coded as $0 ; n=102$ ) and transformation did not improve its distribution, this variable was not transformed for further analyses. A low-grade inflammation (LGI) score was calculated by averaging the Z-scores [i.e. (individuals' observed values - population mean)/SD] of the six (In transformed) inflammatory markers (IL-6, IL-8, TNF- $\alpha$, hs-CRP, SAA, sICAM-1) (29). General characteristics of the study population were compared across categories of glucose metabolism status using analysis of variance (ANOVA) for continuous variables and $\chi^{2}$-test for discrete variables.

Associations between markers of iron metabolism (ferritin, transferrin, serum iron, NTBI, and transferrin saturation) and metabolic variables (adipocyte IR, NEFAs, adiponectin, HOMA2-IR) were examined with the use of multiple linear regression analyses. For In-transformed outcome variables (i.e. adipocyte IR, adiponectin, and HOMA2-IR), regression coefficients were converted into \% change in the original variable (e.g. \% increase in adipocyte IR per $10 \mu \mathrm{g} / \mathrm{I}$ increase in ferritin). All analyses were adjusted for age, sex, glucose metabolism status, prior CVD, eGFR, smoking status, alcohol consumption, dietary energy intake, physical activity, family history of T2DM, use of medication, waist circumference, and the LGI score. In addition, analyses with serum ferritin and transferrin were mutually adjusted for each other to account for negative confounding, since they are both independently associated with insulin resistance despite their negative reciprocal correlation $(11,12)$. Appreciation of normal probability plots of residuals confirmed that, in all models (including those with NTBI as determinant), the assumption of normality was not violated.

To examine whether oxidative stress could explain (part of) the associations, if present, between iron markers and IR, we examined whether associations between iron markers and IR were significantly mediated by MDA or TAS. MDA or TAS was added to the fully adjusted regression models, and the 'mediated effect' was quantified by the absolute attenuation of the regression coefficient. Confidence intervals around this 'mediated effect' were derived from bootstrapping (with 5000 generated datasets), as previously described in detail (19).

We also investigated whether the associations differed between sexes by adding interaction terms between iron parameters and sex to the (fully adjusted) models. We found no such effect-modification by sex and therefore all results are presented for men and women combined. A two-sided p-value of $<0.05$ was considered statistically significant. All analyses were performed using the Statistical Package for Social Sciences (SPSS) for Windows, version 17.0 (SPSS Inc., Chicago, IL, USA). 


\section{Results}

\section{Study population}

General characteristics of the study population are shown in Table 3.2.1. Adipocyte IR increased and plasma adiponectin decreased across the spectrum of normal glucose metabolism (NGM), impaired glucose metabolism (IGM) and T2DM (both $p<0.001$ ). In addition, median serum ferritin and mean serum transferrin increased from NGM to IGM and T2DM $(p<0.001$ and $p=0.050$ respectively), along with TAS $(p<0.001)$. No significant differences were observed for serum iron, NTBI, transferrin saturation, or MDA.

\section{Associations of iron metabolism with adipocyte insulin resistance, NEFAs and adiponectin}

After adjustment for age, sex, glucose metabolism status, and each other, serum ferritin and transferrin were significantly associated with higher adipocyte IR: $\beta=1.38 \%$ increase in adipocyte IR per $10 \mu \mathrm{g} / \mathrm{l}$ increase in ferritin (95\% Cl: $1.01-1.76)$, and $\beta=5.26 \%$ per $0.1 \mathrm{~g} / \mathrm{l}$ increase in transferrin $(95 \% \mathrm{Cl} 3.77-6.78$ (Table 3.2.2, model 2). These associations were slightly attenuated, but remained significant after adjustment for covariates such as prior CVD, eGFR, smoking status, alcohol consumption, dietary energy intake, physical activity, family history of T2DM, use of medication (model 3), waist circumference (model 4$)$ and LGI score (model 5): $\beta=1.00 \%(95 \% \mathrm{Cl} 0.66-1.34)$ and $\beta=4.18 \%(95 \% \mathrm{Cl} 2.88-5.50)$, respectively. Serum iron and NTBI were initially not associated with adipocyte IR (Table 3.2.2, models 1-3). However, after adjustments for potential negative confounding variables, i.e. waist circumference and the LGI score (model 5), both were significantly associated with higher adipocyte IR: $\beta=1.36 \%$ per $\mu \mathrm{mol} / \mathrm{I}$ increase in serum iron $(95 \% \mathrm{Cl} 0.61-2.12)$, and $\beta=5.14 \%$ per $\mu \mathrm{mol} / \mathrm{l}$ increase in NTBI (95\% CI 1.88-8.52). Transferrin saturation was inversely associated with adipocyte IR in analyses adjusted for age, sex and glucose metabolism status, but this was completely attenuated after adjustment for covariates (Supplementary Table S3.2.1).

To underscore that the observed associations with adipocyte IR indeed reflect associations with NEFAs (and thus potentially reduced suppression of the release of NEFAs from the adipose tissue), and not just hyperinsulinemia, associations of iron metabolism markers with fasting plasma NEFAs are also shown in Table 3.2.2. After adjustment for covariates, ferritin, transferrin, serum iron, and NTBI were all associated with higher NEFAs: $\beta=1.94 \mu \mathrm{mol} / \mathrm{I}$ increase in NEFAs per $10 \mu \mathrm{g} / \mathrm{l}$ increase in ferritin (95\% Cl 0.71-3.16), $\beta=6.07 \mu \mathrm{mol} / \mathrm{l}$ per $0.1 \mathrm{~g} / \mathrm{l}$ increase in transferrin $(95 \% \mathrm{Cl} 1.46-10.7)$, $\beta=3.99 \mu \mathrm{mol} / \mathrm{l}$ per $\mu \mathrm{mol} / \mathrm{l}$ increase in serum iron $(95 \% \mathrm{Cl} 1.36-6.61)$, and $\beta=15.7 \mu \mathrm{mol} / \mathrm{l}$ per $\mu \mathrm{mol} / \mathrm{l}$ increase in NTBI $(95 \% \mathrm{Cl} 4.60-26.8)$. Moreover, additional adjustment for insulin did not materially affect these associations: $\beta=2.10(95 \% \mathrm{Cl} 0.83-3.37)$ for ferritin, 
$\beta=6.61(95 \% \mathrm{Cl} 1.87-11.4)$ for transferrin, $\beta=3.99(95 \% \mathrm{Cl} 1.36-6.63)$ for serum iron, and $\beta=15.7(95 \% \mathrm{Cl} 4.57-26.8)$ for NTBI.

Table 3.2.1 General characteristics of the study population $(n=492)$.

\begin{tabular}{|c|c|c|c|c|}
\hline & \multicolumn{3}{|c|}{ Glucose metabolism status } & \multirow[b]{2}{*}{ p-value } \\
\hline & NGM (n=268) & IGM (n=114) & $\mathrm{DM}(\mathrm{n}=110)$ & \\
\hline Age (years) & $58.8 \pm 7.3$ & $60.0 \pm 6.4$ & $60.8 \pm 6.4$ & 0.025 \\
\hline Male sex (\%) & 59 & 58 & 64 & 0.608 \\
\hline Prior CVD (\%) & 23 & 30 & 40 & 0.003 \\
\hline Current smoker (\%) & 21 & 18 & 20 & 0.815 \\
\hline Family history of T2DM (\%) & 35 & 45 & 52 & 0.007 \\
\hline Energy intake (MJ/d) & $9.48 \pm 2.7$ & $9.07 \pm 2.9$ & $8.62 \pm 2.6$ & 0.020 \\
\hline Alcohol consumers (\%) & 93 & 91 & 86 & 0.165 \\
\hline Alcohol $(g / d)$ in consumers & $10.1[2.7-25.2]$ & $9.5[1.4-28.6]$ & $10.1[2.9-27.9]$ & 0.804 \\
\hline Physical activity $\left(10^{3} \bullet\right.$ METs/wk) & $7.37 \pm 4.5$ & $6.17 \pm 4.3$ & $6.60 \pm 4.1$ & 0.036 \\
\hline \multicolumn{5}{|l|}{ Use of medication } \\
\hline Antihypertensive (\%) & 27 & 42 & 58 & $<0.001$ \\
\hline Lipid-lowering (\%) & 16 & 18 & 26 & 0.084 \\
\hline Glucose-lowering (\%) & 0 & 3 & 49 & $<0.001$ \\
\hline Body mass index $\left(\mathrm{kg} / \mathrm{m}^{2}\right)$ & $27.6 \pm 3.9$ & $28.9 \pm 4.3$ & $30.2 \pm 4.6$ & $<0.001$ \\
\hline Waist circumference $(\mathrm{cm})$ & $96.1 \pm 10.9$ & $100.8 \pm 11.8$ & $104.8 \pm 11.6$ & $<0.001$ \\
\hline Fasting glucose (mmol/l) & $5.3[5.0-5.5]$ & $6.0[5.5-6.3]$ & $7.4[6.9-8.6]$ & $<0.001$ \\
\hline Fasting insulin (pmol/l) & $52[41-68]$ & 67 [45 - 99] & $84[60-121]$ & $<0.001$ \\
\hline NEFAs $(\mu \mathrm{mol} / \mathrm{l})$ & $481 \pm 157$ & $553 \pm 196$ & $583 \pm 196$ & $<0.001$ \\
\hline Adipocyte IR & $24[17-33]$ & $34[22-61]$ & $46[33-71]$ & $<0.001$ \\
\hline HOMA2-IR & $0.98[0.78-1.28]$ & $1.28[0.88-1.91]$ & $1.71[1.22$ - 2.47] & $<0.001$ \\
\hline Adiponectin $(\mu \mathrm{g} / \mathrm{ml})$ & $8.1[6.2-10.9]$ & $6.9[5.6-9.7]$ & $5.7[4.2-8.0]$ & $<0.001$ \\
\hline Ferritin $(\mu \mathrm{g} / \mathrm{l})$ & $129[70-227]$ & $162[80-256]$ & $216[127-340]$ & $<0.001$ \\
\hline Transferrin (g/l) & $2.50 \pm 0.34$ & $2.58 \pm 0.34$ & $2.57 \pm 0.37$ & 0.050 \\
\hline Serum iron $(\mu \mathrm{mol} / \mathrm{l})$ & $18.8 \pm 6.2$ & $19.4 \pm 5.2$ & $18.7 \pm 6.2$ & 0.628 \\
\hline NTBI $(\mu \mathrm{mol} / \mathrm{l})$ & $1.11[0.22-2.28]$ & $1.21[0.23-1.90]$ & $1.12[0.12-2.11]$ & 0.463 \\
\hline Transferrin saturation (\%) & $35 \pm 12$ & $35 \pm 11$ & $33 \pm 11$ & 0.283 \\
\hline MDA $(\mu \mathrm{mol} / \mathrm{l})$ & $0.18 \pm 0.05$ & $0.18 \pm 0.05$ & $0.17 \pm 0.04$ & 0.221 \\
\hline TAS (mmol/l) & $1.06 \pm 0.10$ & $1.08 \pm 0.10$ & $1.13 \pm 0.10$ & $<0.001$ \\
\hline IL-6 (pg/ml) & $1.51[1.00-2.25]$ & $1.54[1.14-2.34]$ & $1.67[1.24-2.39]$ & 0.072 \\
\hline $\mathrm{IL}-8$ (pg/ml) & $4.10[3.40-5.00]$ & $4.30[3.56-5.50]$ & $4.99[4.09-6.34]$ & $<0.001$ \\
\hline TNF- $\alpha(p g / m l)$ & $6.15[5.21-7.53]$ & $6.11[5.29-7.61]$ & $6.30[5.40-7.56]$ & 0.757 \\
\hline hs-CRP(mg/l) & $1.71[0.86-3.21]$ & $2.09[1.00-4.38]$ & $3.06[1.42-6.42]$ & $<0.001$ \\
\hline $\mathrm{SAA}(\mathrm{mg} / \mathrm{l})$ & $1.29[0.78-2.09]$ & $1.35[0.89-2.61]$ & $1.64[1.02-3.06]$ & 0.013 \\
\hline sICAM-1 ( $\mu \mathrm{g} / \mathrm{l})$ & 203 [175 - 237] & 212 [188 - 249] & 231 [196 - 266] & $<0.001$ \\
\hline LGI score & $-0.12 \pm 0.61$ & $0.03 \pm 0.59$ & $0.25 \pm 0.64$ & $<0.001$ \\
\hline eGFR $\left(\mathrm{ml} / \mathrm{min} / 1.73 \mathrm{~m}^{2}\right)$ & $84 \pm 16$ & $85 \pm 16$ & $90 \pm 21$ & 0.005 \\
\hline
\end{tabular}

Data are expressed as mean $\pm \mathrm{SD}$, median [interquartile range] or percentages; NGM: normal glucose metabolism; IGM, impaired glucose metabolism; T2DM: type 2 diabetes mellitus; CVD: cardiovascular disease; NEFAs: non-esterified fatty acids; HOMA2-IR: homeostasis model assessment insulin resistance; Adipocyte IR: adipocyte insulin resistance index; NTBI: non-transferrin-bound iron; MDA: malondialdehyde; TAS: total antioxidant status; IL-6: interleukin-6; IL-8: interleukin-8; TNF- $\alpha$ : tumour necrosis factor- $\alpha$; hs-CRP: high sensitivity $C$-reactive protein; SAA: serum amlyoid A; sICAM-1: soluble inter-cellular adhesion molecule-1; LGI score: low-grade inflammation score; eGFR: estimated glomerular filtration rate. 


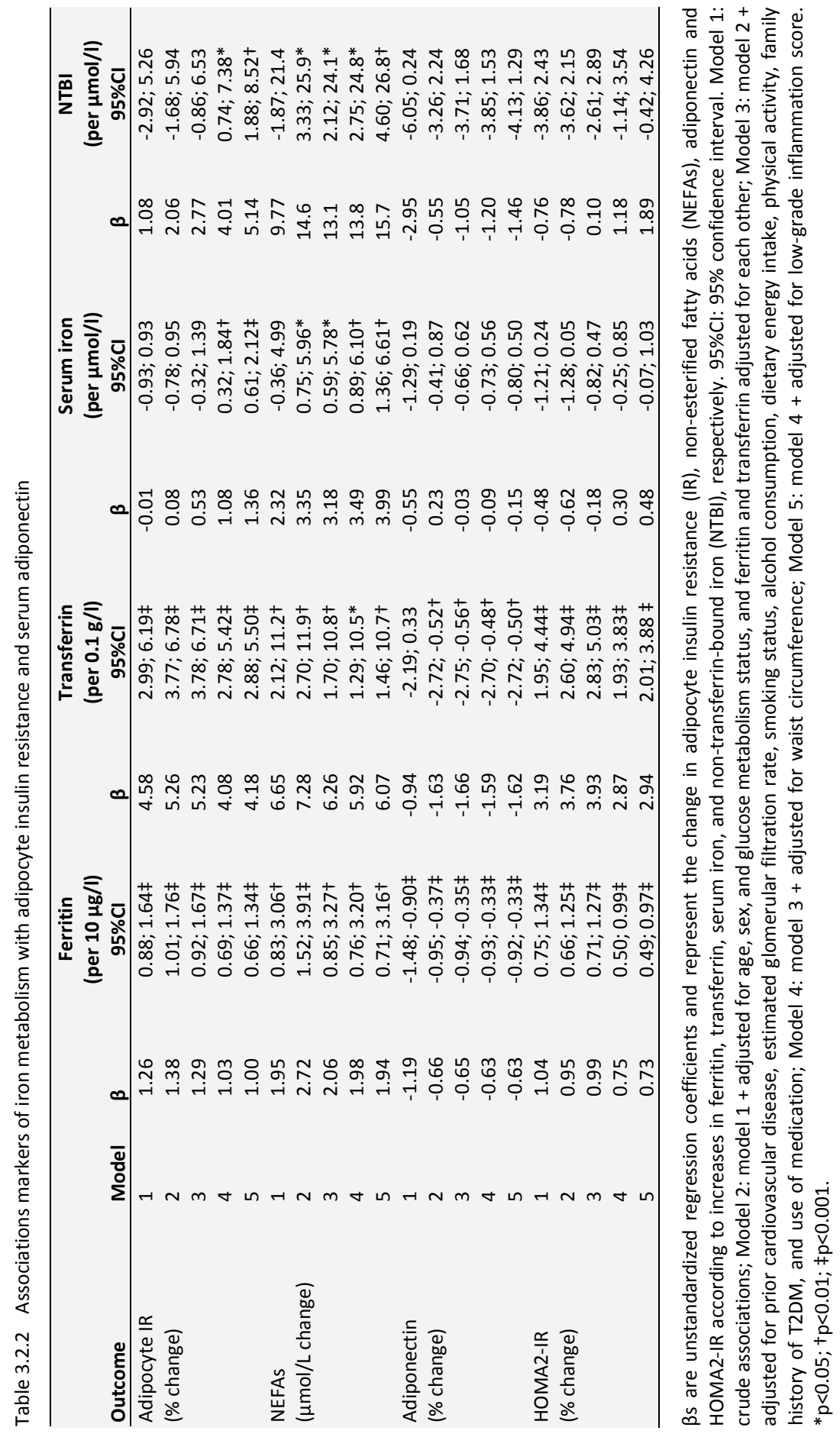


Finally, both ferritin and transferrin were inversely associated with plasma adiponectin in fully adjusted models: $\beta=-0.63 \%$ decrease in adiponectin per $10 \mu \mathrm{g} / \mathrm{l}$ increase in ferritin $(95 \% \mathrm{Cl}-0.92$ to -0.33$)$, and $\beta=-1.62 \%$ per $0.1 \mathrm{~g} / \mathrm{l}$ increase in transferrin $(95 \% \mathrm{Cl}$ -2.72 to -0.50 ) (Table 3.2.2, model 5). In contrast with ferritin and transferrin, serum iron and NTBI were not significantly associated with lower plasma adiponectin.

\section{Associations of iron metabolism with HOMA2-IR}

Serum ferritin and transferrin were both significantly associated with higher HOMA2-IR after adjustment for age, sex, glucose metabolism status, and each other: $\beta=0.95 \%$ increase in HOMA2-IR per $10 \mu \mathrm{g} / \mathrm{l}$ increase in ferritin $(95 \% \mathrm{Cl} 0.66-1.25)$, and $\beta=3.76 \%$ per $0.1 \mathrm{~g} / \mathrm{l}$ increase in transferrin $(95 \% \mathrm{Cl} 2.60-4.94)$ (Table 3.2.2, model 2). After adjustment for covariates, both ferritin $(\beta=0.73 \% ; 95 \% \mathrm{Cl} 0.49-0.97)$ and transferrin $(\beta=2.94 \% ; 95 \% \mathrm{Cl} 2.01-3.88)$ remained significantly associated with HOMA2-IR. In fully adjusted models serum iron and NTBI were also positively associated with HOMA2-IR, although these were not statistically significant (Table 3.2.2, model 5). Finally, transferrin saturation was inversely associated with HOMA2-IR (Supplementary Table S3.2.1, models 1-2) but this association was attenuated and no longer significant after adjustments for waist circumference and LGI (models 4-5).

\section{Mediation by markers of oxidative stress}

We also examined whether the observed associations of ferritin, transferrin, serum iron, and NTBI with adipocyte IR and HOMA2-IR were mediated by MDA or TAS. Fully adjusted associations (Table 3.2.2; model 5$)$ of ferritin $(\beta=1.00 \%, 95 \% \mathrm{Cl} 0.66-1.34)$ and transferrin $(\beta=4.18 \%, 95 \% \mathrm{Cl} 2.88-5.50)$ with adipocyte IR were significantly mediated by TAS, although only to a limited extent. The effect size of the association between ferritin and adipocyte IR was attenuated by $\beta=0.06(95 \% \mathrm{Cl} 0.00-0.16)$ when TAS was included in the regression model, and for transferrin, attenuation by TAS was $\beta=0.21$ (95\% Cl 0.01-0.53). Likewise, fully adjusted associations (Table 3.2.2; model 5) of ferritin $(\beta=0.73 \%, 95 \% \mathrm{Cl} 0.49-0.97)$ and transferrin $(\beta=2.94 \%, 95 \% \mathrm{Cl} 2.01-3.88)$ with HOMA2-IR were significantly mediated by TAS, with mediated portions of $\beta=0.05$ $(95 \% \mathrm{Cl}$ 0.01-0.13) for ferritin, and $\beta=0.18(95 \% \mathrm{Cl} 0.03-0.45)$ for transferrin. No significant mediation by TAS was found for associations with iron or NTBI (data not shown). In addition, there was no significant mediation by MDA in any of the associations (data not shown).

\section{Additional analyses}

All analyses, as presented above, excluded subjects with possible haemochromatosis defined based on the combination of elevated transferrin saturation ( $>45 \%$ ) and elevated ferritin. Excluding all subjects with elevated transferrin saturation $(n=68)$ did 
not appreciably change the associations between ferritin, transferrin, serum iron, and NTBI with metabolic outcomes, however (data not shown).

To account for the possibility of markers of iron metabolism being affected by the presence of infectious or inflammatory diseases at the time of blood sampling, we also repeated all the analyses excluding subjects with serum hs-CRP $>10 \mathrm{mg} / \mathrm{l}(\mathrm{n}=41)$. This did not materially change the associations of ferritin, transferrin, serum iron and NTBI with metabolic outcomes (data not shown). In addition, similar associations were observed after stringent exclusion of subjects with any self-reported (history of) pulmonary, renal, gastro-intestinal, thyroid, rheumatic disease or cancer $(n=173$, data not shown).

\section{Conclusions}

The present study has four novel findings. First, we showed that iron metabolism was robustly associated with adipocyte IR. Ferritin, transferrin, serum iron as well as NTBI were associated with adipocyte IR in humans, also after taking a broad range of covariates into account. Second, these findings were further corroborated by associations of these markers with plasma NEFAs, and by inverse associations between serum ferritin and transferrin with adiponectin. Third, adjustment for inflammatory markers or exclusion of subjects with elevated hs-CRP did not attenuate these associations, but in some cases, even increased the magnitude of the associations. Finally, associations of ferritin and transferrin with adipocyte IR and HOMA2-IR were significantly mediated by TAS. Taken together, these results suggest that body iron stores and/or iron metabolism may be involved in the development of insulin resistance not only in liver or muscle, but also in adipocytes.

The observed associations of iron parameters with adipocyte IR and adiponectin may be important, since it is thought that adipocyte IR or adipocyte dysfunction is one of the first events in the pathogenesis of T2DM (31). The results of our and other epidemiological studies are supported by several in vitro and animal studies. Incubation of adipocytes with iron or transferrin resulted in increased lipolysis, and decreased insulin-stimulated glucose transport $(13,14)$. Moreover, in mice, an iron-enriched diet caused iron accumulation in visceral fat together with IR (15), and an iron-restricted diet led to lower free fatty acids and triglycerides (16).

Previous epidemiological studies have shown associations of ferritin and transferrin with HOMA-IR, serum triglycerides (TG), high-density lipoprotein (HDL), or the TG/HDL ratio in agreement with these findings (1,9-12). However, HOMA-IR and these lipid variables do not necessarily reflect adipocyte $I R$, and these studies did not adjust for potential confounding variables as smoking, diet, physical activity, and family history of T2DM. The present study confirms and extends previous in vitro data and epidemiological studies by showing firstly that both ferritin and transferrin were 
associated with HOMA-IR, independently of each other and several potential metabolic confounders, and secondly by showing that ferritin, transferrin, iron, and NTBI were independently associated with an adipocyte IR index. Although adipocyte IR and HOMA2-IR were both derived from fasting insulin levels, ferritin, transferrin, iron, and NTBI were also independently associated with fasting NEFAs, even after adjustment for insulin. This suggests that, although highly correlated, the adipocyte IR and HOMA2-IR indices do not represent the same site of insulin resistance, and that our findings indeed concern adipocyte lipolysis.

In addition, ferritin and transferrin were inversely associated with plasma adiponectin, in agreement with previous studies $(7,17)$. Some authors have suggested that the association of ferritin with adiponectin may be explained by abdominal fat (7), but adjustment for obesity and related LGI did not affect the observed associations in our and one previous study (17). The potential inhibitory role of iron on adiponectin production and secretion is yet unclear, but may be interesting. Low adiponectin levels are associated with the development of T2DM, and have been suggested to be a specific marker of adipose tissue dysfunction $(7,32)$. Therefore, the observed associations of ferritin and transferrin with both adipocyte IR and low adiponectin levels support the hypothesis that iron metabolism may influence adipose tissue function.

In contrast to serum ferritin, published data on serum total iron and NTBI levels in obesity or T2DM are scarce. One study has shown that NTBI levels were significantly higher in patients with T2DM than in healthy controls (33). We did not observe this difference in our study, but it should be noted that our population as a whole had an increased metabolic risk, and that median NTBI levels were relatively high even in the NGM group. Of interest, the associations of both total serum iron and NTBI with adipocyte IR emerged only after adjustment for waist circumference and the LGI score. The increases in magnitude of associations between serum iron and NTBI with adipocyte IR or HOMA-IR across models 1-5 may be attributed to negative confounding of obesity and LGI on these associations. Obesity and its related LGI may increase hepcidin production (34), which in turn lowers circulating iron levels (by decreased intestinal absorption and decreased release from macrophages) (35). In regression analyses that are not adjusted for obesity and LGI, the "true" positive association between iron / NTBI levels and adipocyte IR is confounded towards the null (models 1-3). After these negative confounding variables had been taken into account, the 'true' associations between iron levels and adipocyte IR became apparent. With regard to adiponectin and HOMA2-IR, associations with serum iron and NTBI were not significant even after taking the negative confounding as described above into account. Still, for HOMA-IR, a trend was observed. One explanation could be that serum iron and NTBI are characterized by more random error (due to circadian fluctuations and day-today variations) than ferritin and transferrin, which may decrease the magnitude of its associations. 
A possible mechanism through which iron may induce insulin resistance is the formation of toxic free radicals. Addition of a free-radical scavenger completely blocked the effect of iron or transferrin on in vitro adipocyte lipolysis (13). Serum ferritin has also been associated with circulating oxidized lipoproteins and advanced oxidation protein products (1), suggesting that iron may cause insulin resistance through induction of oxidative stress. In addition, we observed significant mediation by TAS in the associations of ferritin and transferrin with adipocyte IR and HOMA2-IR. The small magnitude of the mediated effect may be due to the fact that TAS is a systemic measure, and does not efficiently reflect local or intracellular oxidative stress. Nevertheless, the significant mediation in our study supports the hypotheses that the association between iron and IR does involve induction of oxidative stress. The association of NTBI levels with adipocyte IR also suggests the involvement of oxidative tress, as NTBI is believed to be a more redox-active form of iron, as it is not so tightly bound to carrier proteins such as transferring $(33,36)$.

In the evaluation of iron metabolism in obesity and T2DM, the concurrent LGI has regularly been implicated as an important confounder, since several markers of iron metabolism (ferritin, transferrin, hepcidin) are also acute-phase proteins $(21,35)$. Remarkably, associations remained unchanged after adjustment for LGI, or after exclusion of subjects with elevated hs-CRP, indicating that (systemic) LGI did not have a confounding role in the associations between iron parameters and insulin resistance. Finally, serum ferritin has been shown to correlate well with the amount of iron removed by phlebotomy, even in subjects with obesity-related LGI (37). Therefore, we conclude that the above associations truly reflect involvement of iron metabolism, and not just LGI.

The present study showed consistent associations of several markers of iron metabolism with systemic markers of insulin resistance in subjects that were carefully metabolically phenotyped. The main limitation of our study is its cross-sectional design, which does not allow us to draw definite conclusions on causality. In a reverse causation scenario, our results could be explained by actions of insulin on iron metabolism. Indeed, insulin has been shown to up-regulate transferrin receptor expression and transferrin production, and therefore iron uptake and ferritin levels $(38,39)$. The reciprocal relation between insulin and iron metabolism is probably very complex, and cannot simply be dissected in epidemiological studies. However, in our study, markers of iron metabolism were also associated with NEFAs, which do not have a (known) reciprocal effect on iron metabolism. In addition, small trials in patients with T2DM or non-alcoholic fatty liver disease have shown that iron depletion by phlebotomy reduces insulin resistance (HOMA2-IR), supporting our concept $(37,40)$. Whether this also holds true for adipocyte insulin resistance remains to be investigated. Finally, our study was conducted in middle-aged and older, Caucasian subjects who were selected on the basis of an increased risk for metabolic 
and cardiovascular disease, and extrapolation to other study populations or other ethnicities should be done with caution.

In conclusion, we have shown that several markers of iron metabolism are associated not only with HOMA2-IR, but also with adipocyte insulin resistance in humans. These findings suggest that body iron stores and/or iron-metabolism-related factors may contribute to the induction of insulin resistance early in the pathogenesis of T2DM. Of note, body iron stores can easily be influenced by low-cost interventions as phlebotomies or dietary interventions. Therefore, iron metabolism, and particularly effects of iron on adipose tissue, represents an interesting feature of the metabolic syndrome that deserves further investigation. 


\section{References}

1. Syrovatka P, Kraml P, Potockova J, Fialova L, Vejrazka M, Crkovska J, Andel M: Relationship between increased body iron stores, oxidative stress and insulin resistance in healthy men. Ann Nutr Metab 2009;54:268-274

2. Beard JL: Iron biology in immune function, muscle metabolism and neuronal functioning. J Nutr 2001;131:568S-579S; discussion 580S

3. Houstis N, Rosen ED, Lander ES: Reactive oxygen species have a causal role in multiple forms of insulin resistance. Nature 2006;440:944-948

4. Pietrangelo A: Hereditary hemochromatosis--a new look at an old disease. N Engl J Med 2004;350: 2383-2397

5. Jiang R, Manson JE, Meigs JB, Ma J, Rifai N, Hu FB: Body iron stores in relation to risk of type 2 diabetes in apparently healthy women. JAMA 2004;291:711-717

6. Jiang R, Ma J, Ascherio A, Stampfer MJ, Willett WC, Hu FB: Dietary iron intake and blood donations in relation to risk of type 2 diabetes in men: a prospective cohort study. Am J Clin Nutr 2004;79:70-75

7. Forouhi NG, Harding AH, Allison M, Sandhu MS, Welch A, Luben R, Bingham S, Khaw KT, Wareham NJ: Elevated serum ferritin levels predict new-onset type 2 diabetes: results from the EPIC-Norfolk prospective study. Diabetologia 2007;50:949-956

8. Haap M, Fritsche A, Mensing HJ, Haring HU, Stumvoll M: Association of high serum ferritin concentration with glucose intolerance and insulin resistance in healthy people. Ann Intern Med 2003; 139:869-871

9. Jehn M, Clark JM, Guallar E: Serum ferritin and risk of the metabolic syndrome in U.S. adults. Diabetes Care 2004;27:2422-2428

10. Lee BK, Kim Y, Kim YI: Association of serum ferritin with metabolic syndrome and diabetes mellitus in the South Korean general population according to the Korean National Health and Nutrition Examination Survey 2008. Metabolism 2011;60:1416-1424

11. Fumeron F, Pean F, Driss F, Balkau B, Tichet J, Marre M, Grandchamp B: Ferritin and transferrin are both predictive of the onset of hyperglycemia in men and women over 3 years: the data from an epidemiological study on the Insulin Resistance Syndrome (DESIR) study. Diabetes Care 2006;29: 2090-2094

12. Vari IS, Balkau B, Kettaneh A, Andre P, Tichet J, Fumeron F, Caces E, Marre M, Grandchamp B, Ducimetiere $P$ : Ferritin and transferrin are associated with metabolic syndrome abnormalities and their change over time in a general population: Data from an Epidemiological Study on the Insulin Resistance Syndrome (DESIR). Diabetes Care 2007;30:1795-1801

13. Rumberger JM, Peters T, Jr., Burrington C, Green A: Transferrin and iron contribute to the lipolytic effect of serum in isolated adipocytes. Diabetes 2004;53:2535-2541

14. Green A, Basile R, Rumberger JM: Transferrin and iron induce insulin resistance of glucose transport in adipocytes. Metabolism 2006;55:1042-1045

15. Dongiovanni P, Ruscica M, Benedan L, Borroni V, Recalcati S, Steffani L, al. e: Dietary iron overload induces visceral adipose tissue insulin resistance associated with hyper-resistinemia, and synergizes with obesity and fatty liver in inducing systemic insulin resistance. J Hepatol 2011;54:S505

16. Cooksey RC, Jones D, Gabrielsen S, Huang J, Simcox JA, Luo B, Soesanto Y, Rienhoff H, Abel ED, McClain DA: Dietary iron restriction or iron chelation protects from diabetes and loss of beta-cell function in the obese (ob/ob lep-/-) mouse. Am J Physiol Endocrinol Metab 2010;298:E1236-1243

17. Ku BJ, Kim SY, Lee TY, Park KS: Serum ferritin is inversely correlated with serum adiponectin level: population-based cross-sectional study. Dis Markers 2009;27:303-310

18. van Greevenbroek MM, Jacobs M, van der Kallen CJ, Vermeulen VM, Jansen EH, Schalkwijk CG, Ferreira I, Feskens EJ, Stehouwer CD: The cross-sectional association between insulin resistance and circulating complement C3 is partly explained by plasma alanine aminotransferase, independent of central obesity and general inflammation (the CODAM study). Eur J Clin Invest 2011;41:372-379

19. Thewissen MM, Damoiseaux JG, Duijvestijn AM, van Greevenbroek MM, van der Kallen CJ, Feskens EJ, Blaak EE, Schalkwijk CG, Stehouwer CD, Cohen Tervaert JW, Ferreira I: Abdominal fat mass is associated with adaptive immune activation: the CODAM Study. Obesity (Silver Spring) 2011;19:1690-1698 
20. Du H, van der AD, van Bakel MM, van der Kallen CJ, Blaak EE, van Greevenbroek MM, Jansen EH, Nijpels G, Stehouwer CD, Dekker JM, Feskens EJ: Glycemic index and glycemic load in relation to food and nutrient intake and metabolic risk factors in a Dutch population. Am J Clin Nutr 2008;87:655-661

21. Swinkels DW, Fleming RE: Novel observations in hereditary hemochromatosis: potential implications for clinical strategies. Haematologica 2011;96:485-488

22. Breuer W, Cabantchik ZI: A fluorescence-based one-step assay for serum non-transferrin-bound iron. Anal Biochem 2001;299:194-202

23. Abdul-Ghani MA, Molina-Carrion M, Jani R, Jenkinson C, Defronzo RA: Adipocytes in subjects with impaired fasting glucose and impaired glucose tolerance are resistant to the anti-lipolytic effect of insulin. Acta Diabetol 2008;45:147-150

24. Vangipurapu J, Stancakova A, Pihlajamaki J, Kuulasmaa TM, Kuulasmaa T, Paananen J, Kuusisto J, Ferrannini E, Laakso M: Association of indices of liver and adipocyte insulin resistance with 19 confirmed susceptibility loci for type 2 diabetes in 6,733 non-diabetic Finnish men. Diabetologia 2011;54:563-571

25. Gastaldelli A, Harrison SA, Belfort-Aguilar R, Hardies L, Balas B, Schenker S, Cusi K: Importance of changes in adipose tissue insulin resistance to histological response during thiazolidinedione treatment of patients with nonalcoholic steatohepatitis. Hepatology 2009;50:1087-1093

26. Groop LC, Bonadonna RC, DelPrato S, Ratheiser K, Zyck K, Ferrannini E, DeFronzo RA: Glucose and free fatty acid metabolism in non-insulin-dependent diabetes mellitus. Evidence for multiple sites of insulin resistance. J Clin Invest 1989;84:205-213

27. Wallace TM, Levy JC, Matthews DR: Use and abuse of HOMA modeling. Diabetes Care 2004;27: 1487-1495

28. Wendel-Vos GC, Schuit AJ, Saris WH, Kromhout D: Reproducibility and relative validity of the short questionnaire to assess health-enhancing physical activity. J Clin Epidemiol 2003;56:1163-1169

29. van Bussel BC, Henry RM, Schalkwijk CG, Ferreira I, Feskens EJ, Streppel MT, Smulders YM, Twisk JW, Stehouwer $\mathrm{CD}$ : Fish consumption in healthy adults is associated with decreased circulating biomarkers of endothelial dysfunction and inflammation during a 6-year follow-up. J Nutr 2011;141:1719-1725

30. Levey AS, Bosch JP, Lewis JB, Greene T, Rogers N, Roth D: A more accurate method to estimate glomerular filtration rate from serum creatinine: a new prediction equation. Modification of Diet in Renal Disease Study Group. Ann Intern Med 1999;130:461-470

31. Iozzo P: Viewpoints on the way to the consensus session: where does insulin resistance start? The adipose tissue. Diabetes Care 2009;32 Suppl 2:S168-173

32. Duncan BB, Schmidt MI, Pankow JS, Bang H, Couper D, Ballantyne CM, Hoogeveen RC, Heiss G: Adiponectin and the development of type 2 diabetes: the atherosclerosis risk in communities study. Diabetes 2004;53:2473-2478

33. Lee DH, Liu DY, Jacobs DR, Jr., Shin HR, Song K, Lee IK, Kim B, Hider RC: Common presence of nontransferrin-bound iron among patients with type 2 diabetes. Diabetes Care 2006;29:1090-1095

34. Cheng HL, Bryant C, Cook R, O'Connor H, Rooney K, Steinbeck K: The relationship between obesity and hypoferraemia in adults: a systematic review. Obes Rev 2011;

35. Means RT, Jr.: Hepcidin and anaemia. Blood Rev 2004;18:219-225

36. Le Lan C, Loreal O, Cohen T, Ropert M, Glickstein H, Laine F, Pouchard M, Deugnier Y, Le Treut A, Breuer W, Cabantchik ZI, Brissot P: Redox active plasma iron in C282Y/C282Y hemochromatosis. Blood 2005; 105:4527-4531

37. Valenti L, Fracanzani AL, Dongiovanni P, Bugianesi E, Marchesini G, Manzini P, Vanni E, Fargion S: Iron depletion by phlebotomy improves insulin resistance in patients with nonalcoholic fatty liver disease and hyperferritinemia: evidence from a case-control study. Am J Gastroenterol 2007;102:1251-1258

38. Davis RJ, Corvera S, Czech MP: Insulin stimulates cellular iron uptake and causes the redistribution of intracellular transferrin receptors to the plasma membrane. J Biol Chem 1986;261:8708-8711

39. O'Riordain MG, Ross JA, Fearon KC, Maingay J, Farouk M, Garden OJ, Carter DC: Insulin and counterregulatory hormones influence acute-phase protein production in human hepatocytes. Am J Physiol 1995;269:E323-330

40. Fernandez-Real JM, Penarroja G, Castro A, Garcia-Bragado F, Hernandez-Aguado I, Ricart W: Blood letting in high-ferritin type 2 diabetes: effects on insulin sensitivity and beta-cell function. Diabetes 2002;51:1000-1004 


\section{Supplementary Table}

Table S3.2.1 Associations of transferrin saturation with insulin resistance and serum adiponectin.

\begin{tabular}{lccc}
\hline & & \multicolumn{2}{c}{$\begin{array}{c}\text { Transferrin saturation } \\
\text { (per 1\%) }\end{array}$} \\
Outcome & Model & $\boldsymbol{\beta}$ & $\mathbf{9 5 \%}$ \\
\hline Adipocyte IR (\% change) & 1 & -0.68 & $-1.16 ;-0.21 \dagger$ \\
& 2 & -0.51 & $-0.96 ;-0.07 *$ \\
& 3 & -0.28 & $-0.72 ; 0.16$ \\
Adiponectin (\% change) & 4 & 0.07 & $-0.32 ; 0.47$ \\
& 5 & 0.21 & $-0.19 ; 0.60$ \\
& 1 & -0.06 & $-0.44 ; 0.33$ \\
& 2 & 0.27 & $-0.06 ; 0.60$ \\
HOMA2-IR (\% change) & 3 & 0.14 & $-0.20 ; 0.47$ \\
& 4 & 0.10 & $-0.24 ; 0.44$ \\
& 5 & 0.07 & $-0.27 ; 0.41$ \\
& 1 & -0.70 & $-1.07 ;-0.33 \ddagger$ \\
& 2 & -0.67 & $-1.02 ;-0.33 \ddagger$ \\
& 3 & -0.45 & $-0.78 ;-0.12 \dagger$ \\
& 4 & -0.14 & $-0.42 ; 0.15$ \\
& 5 & -0.05 & $-0.34 ; 0.23$ \\
\hline
\end{tabular}

$\beta$ s are unstandardized regression coefficients and represent the change in adipocyte insulin resistance (IR), adiponectin, and HOMA2-IR per 1 unit (i.e. 1\%) increase in transferrin saturation. 95\% Cl: 95\% confidence interval. Model 1: crude associations; Model 2: model $1+$ adjusted for age, sex, and glucose metabolism status; Model 3: model 2 + adjusted for prior cardiovascular disease, estimated glomerular filtration rate, smoking status, alcohol consumption, dietary energy intake, physical activity, family history of T2DM, and use of medication; Model 4: model 3 + adjusted for waist circumference; Model 5: model 4 + adjusted for lowgrade inflammation score. ${ }^{*} p<0.05 ; \uparrow p<0.01 ; \neq p<0.001$. 


\section{Chapter 3}

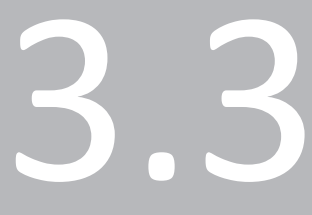

Iron metabolism is prospectively associated with insulin resistance and glucose intolerance over

a 7-year follow-up period

Nick Wlazlo, Marleen MJ van Greevenbroek, Isabel Ferreira, Eugene HJM Jansen, Edith JM Feskens, Carla JH van der Kallen, Casper G Schalkwijk, Bert Bravenboer, Coen DA Stehouwer 


\section{Abstract}

\section{Objective}

Several markers of iron metabolism have been associated with insulin resistance (IR) and type 2 diabetes mellitus (T2DM) in cross-sectional studies. However, prospective data on these associations are scarce, and it is currently unclear in which tissues iron metabolism may contribute to IR. Therefore, we investigated whether markers of iron metabolism were associated with IR in muscle, liver, and adipocytes, and with glucose intolerance over a 7-year follow-up period.

\section{Research design and methods}

Serum ferritin, transferrin, total iron, non-transferrin-bound iron (NTBI), and transferrin saturation were determined at baseline of a prospective cohort study in 509 individuals (60\% men, age $59 \pm 6.9$ years, BMI 28.5 \pm 4.3 ). Both at baseline and after a 7-year follow-up $(n=386)$, measures of glucose, insulin (during glucose tolerance tests), and non-esterified fatty acids were obtained. Using generalized estimating equations, we investigated associations between baseline iron markers and indices of muscle, liver, and adipocyte insulin resistance, as well as glucose intolerance, over the 7-year period.

\section{Results}

Over a 7-year period, baseline serum ferritin (per $10 \mu \mathrm{g} / \mathrm{l}$ increase) was positively associated with HOMA2-IR ( $\beta=0.77 \%$ [95\% Cl 0.50-1.03]), hepatic IR ( $\beta=0.39 \%$ [0.23-0.55]), adipocyte IR ( $\beta=1.00 \%$ [0.65-1.35]), and $A \cup C_{\text {glucose }}(\beta=0.32 \%[0.18-0.46])$ after adjustment for several covariates, including inflammatory markers (all $p<0.001$ ). Similarly, serum transferrin (per $0.1 \mathrm{~g} / \mathrm{l}$ ) was associated with HOMA2-IR ( $\beta=2.66 \%$ [1.55-3.78]), hepatic IR $(\beta=1.16 \%[0.47-1.85])$, adipocyte IR $\left(\beta=3.75 \%\right.$ [2.27-5.25]), and $A \cup C_{\text {glucose }}(\beta=1.35 \%$ [0.74-1.96]) over 7 years.

\section{Conclusions}

Iron metabolism and related factors may contribute to IR in muscle, liver and adipocytes, eventually leading to impaired glucose metabolism and hyperglycemia. 


\section{Introduction}

Iron is a major catalyst in the production of highly reactive hydroxyl radicals $(1,2)$. These reactive oxygen species are believed to play an important role in the induction of insulin resistance (IR) early in the pathogenesis of type 2 diabetes mellitus (T2DM) (3). In agreement, T2DM is very common in iron overload conditions such as hereditary haemochromatosis (4). Moreover, there is increasing evidence that body iron stores within the normal range may contribute to the development of T2DM in the general population. Nutritional studies have shown that dietary (haem) iron intake was independently associated with incident $\operatorname{T2DM}(5,6)$. In addition, serum ferritin, the most reliable marker of body iron stores, has been associated with IR and T2DM in several cross-sectional studies (1,7-12). On the other hand, serum ferritin and hepatic iron load have not been clearly associated with beta cell function $(8,13)$, indicating that the contribution of iron metabolism and iron metabolism-related factors in the pathophysiology of T2DM may be primarily related to the development of IR.

Despite reported associations between total body iron stores and overall IR, it is currently unclear whether these associations pertain to specific tissues. Most epidemiological studies have only reported associations with either homeostasis model assessment insulin resistance (HOMA-IR) or whole-body glucose disposal (1,8-12), which mainly reflect muscle IR, and probably also hepatic IR. Indeed, hepatic iron content, as measured by magnetic resonance imaging, was recently shown to be associated with an IR index derived from an oral glucose tolerance test, suggesting that iron induces IR specifically at the level of the liver (13). Besides, we have recently shown that several markers of iron metabolism were cross-sectionally associated with a validated adipocyte IR index, and inversely with plasma levels of adiponectin, indicating that iron may contribute to the induction of IR in adipose tissue as well (14).

Prospective data on the relation between markers of iron metabolism and IR are, however, scarce. One nested case-control study showed that baseline ferritin and transferrin were associated with increases in insulin and new-onset hyperglycaemia over 3 years (15), and with a high HOMA-IR at 6 years (16). However, these studies did not adjust for regular risk factors for IR such as dietary energy intake, physical activity, smoking status, or a positive family history of T2DM. In addition, only HOMA2-IR was used as a measure of IR.

To further clarify the contribution of iron metabolism in the development of IR, we examined whether baseline markers of iron stores and iron metabolism were associated with (changes in) IR in muscle, liver, and adipocytes over a 7-year follow-up period in a prospective cohort study with extensive (metabolic) characterization of participants. In addition, we investigated associations of these markers with (progression of) glucose intolerance. 


\section{Research design and methods}

\section{Subjects and study design}

The Cohort on Diabetes and Atherosclerosis Maastricht (CODAM) study is a prospective, observational study on, among others, the natural progression of IR and glucose tolerance. A total of 574 individuals were selected from a large population-based cohort as described in detail elsewhere $(17,18)$, and were extensively characterized at baseline with regard to their lifestyle, and cardiovascular and metabolic profile during two visits to the University's metabolic research unit. After a median of 7.0 years [IQR 6.9-7.1], 495 subjects participated in the follow-up measurements (attrition rate 14\% - Figure 3.3.1). The CODAM study was approved by the Medical Ethical Committee of the Maastricht University Medical Centre, and all subjects gave written informed consent.

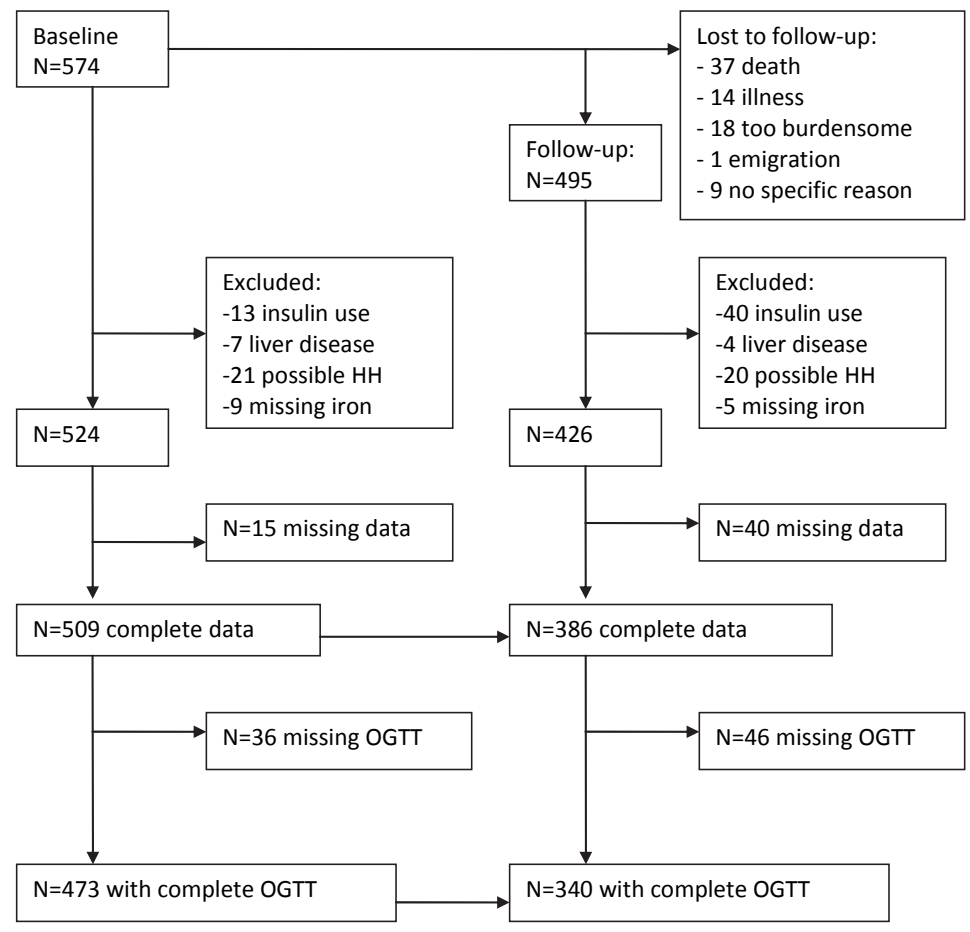

Figure 3.3.1 Flow chart of study participants. 
For the present study, we excluded participants with self-reported (history of) liver disease $(n=7)$ and subjects on insulin therapy ( $n=13$ at baseline, $n=40$ at follow-up). To exclude possible hereditary haemochromatosis, we excluded subjects with elevated serum ferritin $(>150 \mu \mathrm{g} / \mathrm{l}$ for premenopausal women, $>400 \mu \mathrm{g} / \mathrm{l}$ for men and postmenopausal women) and elevated transferrin saturation ( $>45 \%)(n=21)$ according to Dutch guidelines (19). Baseline data were complete in 509 subjects, except for OGTT-derived data, which were complete in 473 subjects. Follow-up data were complete in 386 subjects, of whom 340 also had complete OGTT data at follow-up. Generally, the 509 selected participants were healthier than the 65 excluded subjects, as indicated by lower adiposity measures, lower glucose, insulin and fatty acid levels, and lower IR indices at baseline in selected participants (data not shown). Similarly, the 386 subjects with follow-up data were healthier than the 188 subjects without (useful) follow-up data, as indicated by lower adiposity measures, lower glucose, insulin, and fatty acid levels, and lower use of anti-hypertensive, lipid-lowering, and glucoselowering medication at baseline in these subjects (data not shown).

\section{Markers of iron stores and iron metabolism at baseline}

Participants were asked to stop their lipid-lowering medication 14 days before the visit, and to stop all other medication the day before the visit. After an overnight fast, venous blood samples were collected for assessment of biomarkers. Ferritin, transferrin, total iron, non-transferrin-bound iron (NTBI) levels, and transferrin saturation were determined in serum at baseline as described previously (14).

\section{Insulin resistance and glucose tolerance at baseline and at follow-up}

A standard $75 \mathrm{~g}$ oral glucose tolerance test (OGTT) was performed with measurement of glucose levels at 0, 30, 60 and $120 \mathrm{~min}$, and, during the follow-up round, also at $15 \mathrm{~min}$. At follow-up, a second measurement of fasting glucose was also obtained from a second visit, and the mean of two fasting glucose levels was used for further analyses. All plasma glucose levels were measured in NaF/KOx plasma with a hexokinase glucose6 phosphate dehydrogenase method (ABX Diagnostics) with inter-assay coefficient of variation $(\mathrm{CV})<5 \%$.

Plasma (EDTA) aliquots were processed on ice, centrifuged at $3000 \mathrm{rpm}$ for $15 \mathrm{~min}$, and stored at $-80^{\circ} \mathrm{C}$ until use. Insulin concentrations at baseline, were determined in fasting EDTA plasma using a two-sided immunoradiometric test (IRMA) using paired monoclonal antibodies (Medgenix Diagnostics) with intra- and inter-assay CV of 5.0 and $6.0 \%$ (17). For the present longitudinal study, we recently performed paired measurements of plasma insulin levels, at baseline and follow-up, at all OGTT time-points mentioned above using a multi-array (MA) detection system based on electro-chemiluminicence technology (SECTOR Imager 2400, Meso Scale Discovery, Gaithersburg, MD, USA) with intra- and inter-assay CV of $2.5 \%$ and $9.7 \%$. As such, 
fasting insulin at baseline was measured twice using two different methods that showed a systematic difference and moderate agreement [intraclass correlation coefficient (ICC) 0.83)]. In order to use all available data and to ensure comparability of baseline and follow-up fasting insulin levels, the previous baseline measurements of fasting insulin (IRMA) were calibrated to the multi-array measurements using Deming regression models as recommended for method comparison $(20,21)$. After calibration, the two insulin variables showed very good agreement (ICC 0.96), and the mean of the two fasting insulin measurements at baseline was used for further analyses.

Fasting non-esterified free fatty acids (NEFAs) at baseline were measured in EDTA plasma using an enzymatic calorimetric NEFA C method (Wako Diagnostics, Richmond, USA) with inter-assay CV $<5 \%$. In addition, paired measurements of NEFAs at baseline and follow-up were performed using the same method. At baseline, the mean of the two measurements was taken for analyses.

Total area under the curves (AUC) for glucose and insulin during the OGTT (for the first 30 minutes and for 120 minutes) were calculated using the trapezoidal method. HOMA2-IR, which has been shown to correlate well with (muscle) glucose disposal in clamp studies (22), was computed using the HOMA2-calculator considering fasting plasma glucose and specific insulin data (http://www.dtu.ox.ac.uk/homacalculator/ index.php). Hepatic insulin resistance (hepatic IR) was estimated using the square root of the product of the areas under the curve for glucose and insulin during the first 30 minutes of the OGTT (i.e. SQRT(glucose ${ }_{0-30}[A U C] \times$ insulin $\left._{0-30}[A U C]\right)$ ) converted to conventional units $([\mathrm{mg} / \mathrm{dl} \cdot \mathrm{hr}] \times$ [ulU/ml.hr]) $(23,24)$. This index has been developed and validated against the product of fasting plasma insulin and endogenous glucose production in clamp studies, and has been postulated to represent hepatic insulin resistance, as it not only takes into account fasting glucose, but also the early suppression of fasting glucose by insulin during the first phase of the OGTT. Adipocyte insulin resistance (adipocyte IR) was calculated as the product of fasting insulin and fasting NEFA concentrations, as described before $(14,25,26)$. This index provides a valid marker of the sensitivity of adipocytes to the anti-lipolytic effect of insulin, since NEFA concentrations and fatty acid turnover in clamp studies are highly correlated, and insulin suppresses lipolysis already at low (i.e. fasting) concentrations (10-20 mU/I) $(26,27)$.

\section{Other covariates}

BMI, waist circumference, smoking behaviour, family history of T2DM (first-degree relatives), dietary calorie intake, mean daily alcohol consumption, physical activity, and use of anti-hypertensive, glucose-lowering, and lipid-lowering medication were determined at baseline and follow-up as previously described $(14,18,28)$. Interleukin- 6 (IL-6), high sensitivity C-reactive protein (hs-CRP), serum amyloid A (SAA), and soluble inter-cellular adhesion molecule-1 (sICAM-1) were determined at baseline using methods described before (17). In addition, paired measurements of baseline and 
follow-up IL-6, hs-CRP, SAA, sICAM-1, interleukin-8 (IL-8), and tumour necrosis factor- $\alpha$ (TNF- $\alpha$ ) were determined in EDTA on a multi-array detection system based on electrochemiluminescence technology (SECTOR Imager 2400, Meso Scale Discovery, USA) $(20,29)$. Similar to the baseline insulin data, the previous baseline measurements of IL-6, hs-CRP, SAA, and SICAM-1 were calibrated to the multi-array measurements, and after calibration (20), the mean of the two measurements at baseline was used for analyses. Glomerular filtration rate (eGFR) and prior CVD at baseline were determined as previously described $(17,30)$. Glucose metabolism status [i.e. normal (NGM), impaired (IGM), or T2DM] was diagnosed according to the WHO 1999 criteria as previously described $(17,18)$.

\section{Statistical analysis}

Variables with a skewed distribution (i.e. HOMA2-IR, hepatic IR, adipocyte IR, fasting glucose, postprandial ( $2 \mathrm{~h})$ glucose, $A U C_{\text {glucose, }}$ all inflammatory markers) were In transformed prior to further analyses. A low-grade inflammation (LGI) score was calculated by averaging the Z-scores [i.e. (individual's observed values - population mean)/SD,] of the six (In transformed) inflammatory markers (IL-6, IL-8, TNF- $\alpha$, hs-CRP, SAA, sICAM-1) (29). To account for the correlation of repeated measurements within subjects, we used generalized estimating equations (GEE) with an exchangeable correlation structure to investigate associations between markers of iron metabolism at baseline and IR and glucose metabolism over a 7-year period (31). All analyses were adjusted for potential confounders age, sex, follow-up time, glucose metabolism status, prior CVD (baseline only), eGFR (baseline only), smoking status, alcohol consumption, dietary energy intake, physical activity, family history of T2DM, use of medication, waist circumference and the LGI score. Analyses with serum ferritin and transferrin were mutually adjusted for each other to account for negative confounding, since they are both independently associated with insulin resistance despite their negative reciprocal correlation (14-16).

In addition, we investigated whether iron markers at baseline were associated with (absolute) changes in IR and glucose metabolism in $n=386$ subjects with 7-year follow-up data using linear regression (31). Because changes in IR and glucose metabolism may have been influenced by lifestyle and/or medical interventions, these analyses were stratified into a "Treated group" $(n=174)$, defined by the presence of T2DM at baseline and/or the use of glucose-lowering and/or lipid-lowering medication at any time, and an "observational" group that did not receive such interventions at all during the follow up $(n=212)$.

We also investigated whether the associations differed between sexes by adding interaction terms for iron parameters and sex to the (fully adjusted) models. We found no such effect modification by sex and therefore all results are presented for men and women combined. A two-sided $p$-value of $<0.05$ was considered statistically significant. 
All analyses were performed using STATA Data Analysis and Statistical Software for Windows, version 9.0/SE (STATACorp LP, Texas, USA).

\section{Results}

\section{Study population}

General characteristics of the study population at baseline $(n=509)$ and follow-up $(n=386)$ are shown in Table 3.3.1. Generally, most variables remained unchanged over the 7-year follow-up period, with indices of IR showing a small decrease over time, while $2 \mathrm{~h}$ glucose and AUC glucose and markers of LGI seemed to increase over time.

\section{Associations of markers of iron metabolism at baseline with whole-body and organ-specific insulin resistance over the 7-year follow-up period}

Baseline serum ferritin and transferrin were significantly associated with HOMA2-IR over a 7-year period: $\beta=1.14 \%(95 \% \mathrm{Cl} 0.81-1.48)$ increase in HOMA2-IR per $10 \mu \mathrm{g} / \mathrm{I}$ increase in ferritin, and $\beta=3.01 \%(95 \% \mathrm{Cl} 1.50-4.55)$ increase in HOMA2-IR per $0.1 \mathrm{~g} / \mathrm{I}$ increase in transferrin (Table 3.3.2; model 1). These associations were somewhat attenuated, but remained significant after adjustment for potential confounders age, sex, follow-up time, glucose metabolism status, ferritin or transferrin, prior CVD (baseline only), eGFR (baseline only), smoking status, alcohol consumption, dietary energy intake, physical activity, family history of T2DM, and use of medication (model 2), and in addition for waist circumference (model 3), and the LGI score (model 4): $\beta=0.77 \%(95 \% \mathrm{Cl} 0.50-1.03)$ for ferritin, and $\beta=2.66 \%(95 \% \mathrm{Cl} 1.55-3.78)$ for transferrin, respectively. Next, ferritin and transferrin were independently associated with the hepatic IR index over the 7-year period: $\beta=0.39 \%(95 \% \mathrm{Cl} 0.23-0.55)$ increase in hepatic IR per $10 \mu \mathrm{g} / \mathrm{l}$ increase in ferritin, and $\beta=1.16 \%(95 \% \mathrm{Cl} 0.47-1.85)$ per $0.1 \mathrm{~g} / \mathrm{I}$ increase in transferrin (Table 3.3.2; model 4). Finally, ferritin and transferrin were associated with adipocyte IR over 7 years: $\beta=1.00 \%(95 \% \mathrm{Cl} 0.65-1.35)$ increase in adipocyte IR per $10 \mu \mathrm{g} / \mathrm{I}$ ferritin, and $\beta=3.75 \%(95 \% \mathrm{Cl} 2.27-5.25)$ per $0.1 \mathrm{~g} / \mathrm{l}$ increase in transferrin (Table 3.3.2; model 4).

In agreement with the associations of ferritin and transferrin, serum total iron and NTBI at baseline were also positively associated with HOMA2-IR, hepatic IR, and adipocyte IR over 7 years in fully adjusted models (Table 3.3.2; model 4), but only the associations of serum total iron and NTBI with adipocyte IR reached statistical significance: $\beta=0.83 \%(95 \% \mathrm{Cl} 0.16-1.51)$ increase in adipocyte IR per $\mu \mathrm{mol} / \mathrm{I}$ serum total iron, and $\beta=3.08 \%(95 \% \mathrm{Cl} 0.01-6.25)$ per $\mu \mathrm{mol} / \mathrm{I}$ NTBI. Transferrin saturation at baseline was inversely associated with HOMA2-IR, hepatic IR, and adipocyte IR in crude models (Supplementary Table S3.3.1; model 1), but these associations were completely attenuated after adjustment for waist circumference and LGI (models $3 \& 4$ ). 
Table 3.3.1 Characteristics of the study population at baseline and follow-up

\begin{tabular}{|c|c|c|c|}
\hline & \multirow{2}{*}{$\begin{array}{c}\mathrm{N}=509 \\
\text { Baseline }\end{array}$} & \multicolumn{2}{|c|}{$\mathrm{N}=386$ with follow-up data } \\
\hline & & Baseline & Follow-up \\
\hline Age (years) & $59 \pm 6.9$ & $59 \pm 6.9$ & $66 \pm 6.9$ \\
\hline Male sex (\%) & 60 & 60 & 60 \\
\hline NGM/IGM/T2DM (\%) & $55 / 23 / 22$ & $60 / 25 / 15$ & $47 / 25 / 28$ \\
\hline Prior CVD (\%) & 28 & 26 & - \\
\hline Current smoker (\%) & 22 & 23 & 16 \\
\hline Family history of T2DM (\%) & 42 & 41 & 48 \\
\hline Energy intake $\left(10^{3} \bullet \mathrm{kcal} / \mathrm{d}\right)$ & $2.19 \pm 0.65$ & $2.22 \pm 0.65$ & $2.02 \pm 0.60$ \\
\hline Alcohol $(g / d)$ & 8.13 [1.34-22.7] & $8.69[1.88-22.6]$ & $8.61[1.47-20.1]$ \\
\hline Physical activity $\left(10^{3} \bullet\right.$ METs/wk) & $6.67 \pm 4.18$ & $6.72 \pm 4.18$ & $6.28 \pm 4.60$ \\
\hline \multicolumn{4}{|l|}{ Use of medication } \\
\hline Antihypertensive (\%) & 38 & 32 & 55 \\
\hline Lipid-lowering (\%) & 18 & 17 & 39 \\
\hline Glucose-lowering (\%) & 11 & 6 & 17 \\
\hline Body mass index $\left(\mathrm{kg} / \mathrm{m}^{2}\right)$ & $28.5 \pm 4.3$ & $28.2 \pm 4.1$ & $27.8 \pm 4.2$ \\
\hline Waist circumference $(\mathrm{cm})$ & $99.1 \pm 11.8$ & $98.1 \pm 11.4$ & $98.9 \pm 11.9$ \\
\hline Fasting glucose (mmol/l) & $5.6[5.2-6.3]$ & $5.5[5.1-6.0]$ & $5.3[5.0-5.9]$ \\
\hline Fasting insulin (pmol/l) & 73 [49-110] & 69 [49-101] & $66[47-98]$ \\
\hline Fasting NEFAs ( $\mu \mathrm{mol} / \mathrm{I})$ & $525 \pm 180$ & $517 \pm 177$ & $458 \pm 170$ \\
\hline $2 \mathrm{~h}$ glucose $(\mathrm{mmol} / \mathrm{l})^{*}$ & $6.7[5.3-9.3]$ & $6.5[5.2-8.8]$ & $7.8[6.0-10.6]$ \\
\hline AUC glucose $(\mathrm{mmol} / / \bullet \mathrm{min})^{*}$ & $1010[860-1228]$ & 983 [840-1202] & 1048 [881-1266] \\
\hline HOMA2-IR & $1.61[1.09-2.43]$ & $1.51[1.07-2.18]$ & $1.43[1.02-2.14]$ \\
\hline Hepatic IR index* & 39 [32-49] & 38 [32-49] & $35[28-44]$ \\
\hline Adipocyte IR index & $36[22-61]$ & $33[22-54]$ & 28 [19-43] \\
\hline Ferritin $(\mu \mathrm{g} / \mathrm{l})$ & $160[77-264]$ & $143[72-255]$ & - \\
\hline Transferrin (g/l) & $2.53 \pm 0.35$ & $2.55 \pm 0.35$ & - \\
\hline Serum iron $(\mu \mathrm{mol} / \mathrm{l})$ & $18.9 \pm 6.0$ & $19.1 \pm 5.9$ & - \\
\hline NTBI ( $\mu \mathrm{mol} / \mathrm{l})$ & $1.13[0.21-2.10]$ & $1.14[0.27-2.12]$ & - \\
\hline Transferrin saturation (\%) & $35 \pm 11$ & $35 \pm 11$ & - \\
\hline IL-6 (pg/ml) & $1.56[1.15-2.28]$ & $1.52[1.10-2.20]$ & $1.54[1.07-2.43]$ \\
\hline IL-8 (pg/ml) & $4.33[3.59-5.45]$ & $4.26[3.50-5.21]$ & $5.07[4.21-6.53]$ \\
\hline TNF- $\alpha(\mathrm{pg} / \mathrm{ml})$ & $6.17[5.27-7.53]$ & $6.19[5.28-7.47]$ & $6.55[5.52-8.31]$ \\
\hline $\mathrm{hs}-\mathrm{CRP}(\mathrm{mg} / \mathrm{l})$ & $2.04[0.97-4.01]$ & $1.86[0.91-3.78]$ & $2.01[0.94-3.95]$ \\
\hline SAA (mg/l) & $1.47[1.01-2.32]$ & $1.45[1.02-2.25]$ & $1.48[0.91-2.76]$ \\
\hline sICAM-1 ( $\mu \mathrm{g} / \mathrm{I})$ & $212[187-244]$ & $207[185-241]$ & 213 [189-249] \\
\hline LGI score & $-0.08 \pm 0.65$ & $-0.14 \pm 0.62$ & $0.04 \pm 0.72$ \\
\hline eGFR (ml/min $\left./ 1.73 \mathrm{~m}^{2}\right)$ & $86 \pm 17$ & $85 \pm 16$ & - \\
\hline
\end{tabular}

Data are expressed as mean $\pm \mathrm{SD}$, median [interquartile range] or percentages; NGM: normal glucose metabolism; IGM, impaired glucose metabolism; T2DM: type 2 diabetes mellitus; CVD: cardiovascular disease; NEFAs: non-esterified fatty acids; AUC glucose: area under the curve for glucose during $75 \mathrm{~g}$ oral glucose tolerance test; HOMA2-IR: homeostasis model assessment insulin resistance; Hepatic IR index: hepatic insulin resistance index; Adipocyte IR: adipocyte insulin resistance index; NTBI: non-transferrin-bound iron; IL-6: interleukin-6; IL-8: interleukin-8; TNF- $\alpha$ : tumour necrosis factor- $\alpha$; hs-CRP: high sensitivity C-reactive protein; SAA: serum amlyoid A; sICAM-1: soluble inter-cellular adhesion molecule-1; LGI score: low-grade inflammation score; eGFR: estimated glomerular filtration rate; * Variables derived from oral glucose tolerance tests (OGTT) were complete in $n=473$ at baseline and $n=340$ at follow-up 


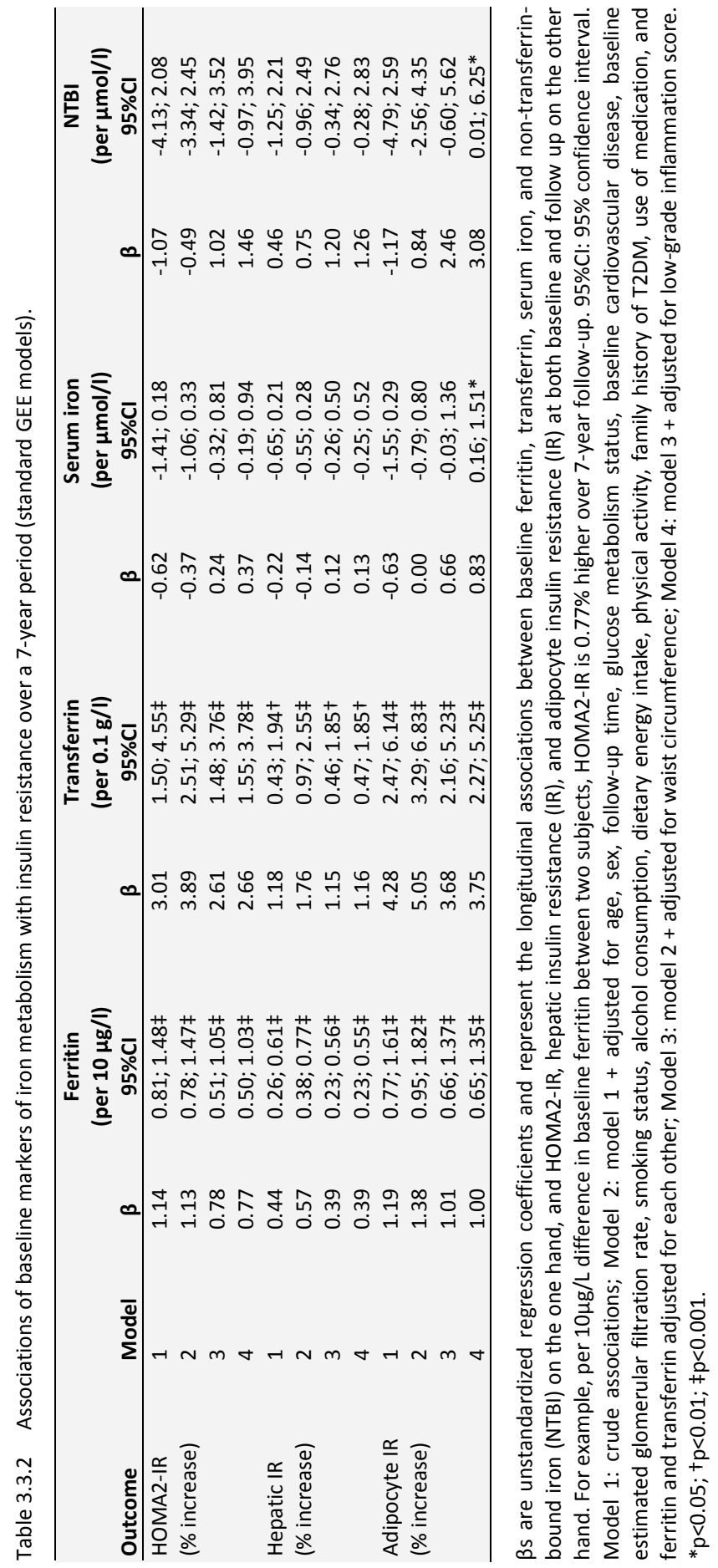




\section{Associations of markers of iron metabolism at baseline with glucose metabolism over the 7-year follow-up period}

After adjustment for all covariates, serum ferritin, but not transferrin, was significantly associated with fasting glucose over the 7-year period: $\beta=0.11 \%(95 \% \mathrm{Cl} 0.03-0.19)$ per $10 \mu \mathrm{g} / \mathrm{l}$ ferritin (Table 3.3.3; model 4). Ferritin and transferrin were both associated with $2 \mathrm{~h}$ glucose over 7 years: $\beta=0.50 \%(95 \% \mathrm{Cl} 0.28-0.71)$ increase in $2 \mathrm{~h}$ glucose per $10 \mu \mathrm{g} / \mathrm{l}$ ferritin, and $\beta=1.98 \%(95 \% \mathrm{Cl} 1.12-2.86)$ per $0.1 \mathrm{~g} / \mathrm{l}$ transferrin (Table 3.3.3; model 4). In addition, both were associated with $A \cup C_{\text {glucose }}$ over 7 years: $\beta=0.32 \%(95 \% \mathrm{Cl} 0.18-0.46)$ increase in $A{ }^{2} C_{\text {glucose }}$ per $10 \mu \mathrm{g} / \mathrm{l}$ ferritin, and $\beta=1.35 \%$ (95\% Cl 0.74-1.96) per $0.1 \mathrm{~g} / \mathrm{l}$ transferrin (Table 3.3.3; model 4). Serum iron and NTBI were positively associated with $2 \mathrm{~h}$ glucose and $\mathrm{AUC}_{\text {glucose }}$ over 7 years, with statistically significant associations between serum iron and $2 \mathrm{~h}$ glucose of $\beta=0.51 \%$ (95\% Cl 0.09-0.94), and between NTBI and $\mathrm{AUC}_{\text {glucose }}$ of $\beta=1.40 \%(95 \% \mathrm{Cl} 0.02-2.81)$ (Table 3.3.3; model 4). No significant associations were observed for transferrin saturation (Supplementary Table S3.3.2).

\section{Associations between markers of iron metabolism at baseline with 7-year changes in whole-body and organ-specific insulin resistance and glucose metabolism}

In addition to the associations derived from standard GEE models, we reanalysed our data using absolute change models. Altogether, no consistent associations were observed between baseline markers of iron metabolism and changes in IR or glucose metabolism during 7-year follow-up (Supplementary Table S3.3.3). These results thus suggest that the associations observed in the GEE models were primarily determined by between-subject effects over the 7-year period. In other words, individuals with higher markers of iron metabolism at baseline had higher levels of IR and glucose intolerance throughout the whole 7-year period, regardless of the changes in these during the 7-year follow-up.

\section{Additional / sensitivity analyses}

All analyses as presented above excluded subjects with possible haemochromatosis defined based on the combination of elevated transferrin saturation (>45\%) and elevated ferritin. A sensitivity analysis excluding all subjects with elevated transferrin saturation ( $n=71$ ) did not appreciably change the associations between of ferritin and transferrin with metabolic outcomes (data not shown). Moreover, in these analyses, the positive associations between iron and HOMA2-IR, adipocyte IR, hepatic IR, and $A \cup C_{\text {glucose }}$ also reached statistical significance (all $p<0.01$ ) (data not shown), as well as the associations of NTBI with hepatic IR and $A U C_{\text {glucose }}$ (both $p<0.01$ ). 


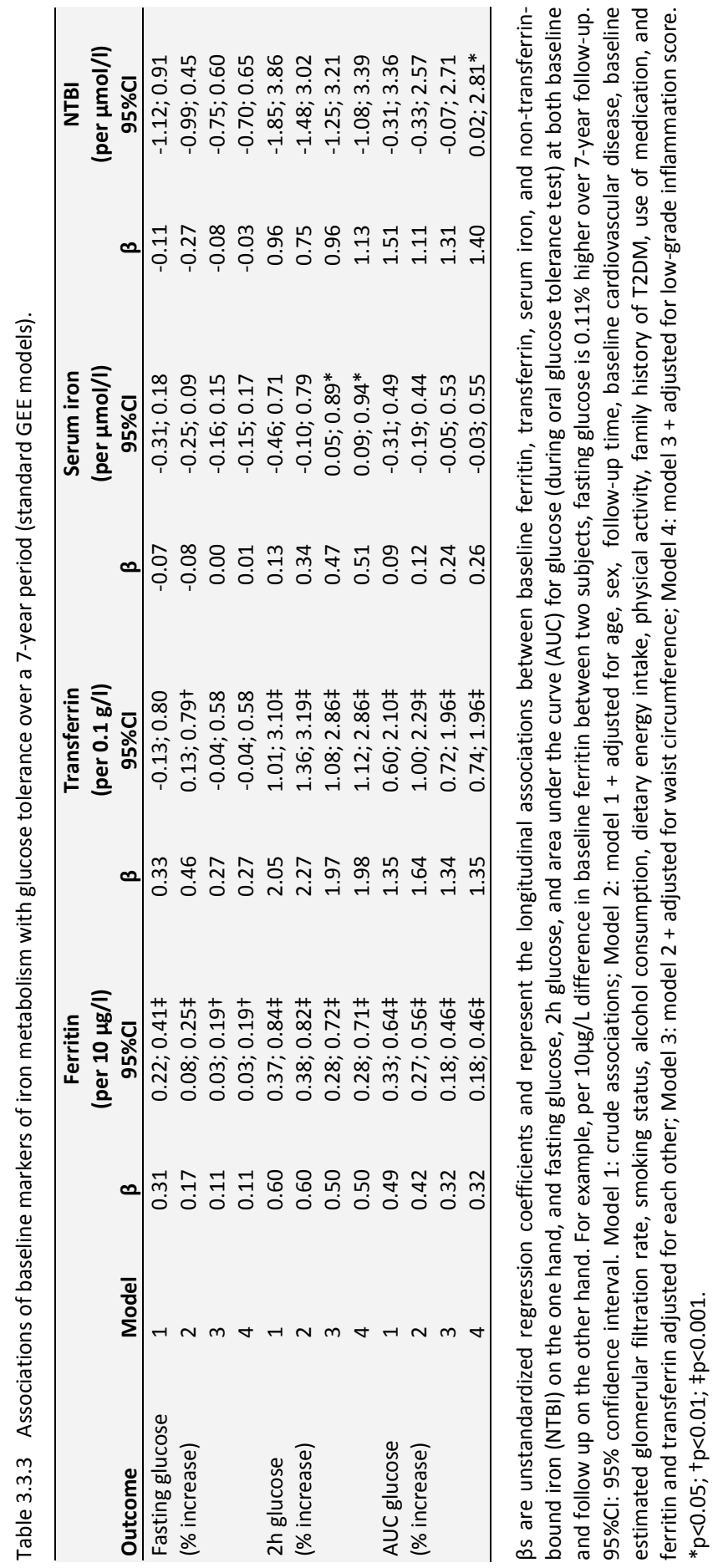


To account for the possibility that markers of iron metabolism were affected by the presence of infectious or inflammatory diseases at the time of blood sampling, we also repeated all the analyses excluding subjects with serum hs-CRP $>10 \mathrm{mg} / \mathrm{l}$ at baseline $(n=36)$. This did not materially change the associations of ferritin, transferrin, serum iron and NTBI with metabolic outcomes (data not shown). In addition, similar associations were observed after stringent exclusion of subjects with any self-reported (history of) pulmonary, renal, gastro-intestinal, thyroid, or rheumatic disease, or cancer at baseline ( $n=180$, data not shown).

\section{Conclusions}

Several studies have shown that serum ferritin is cross-sectionally associated with HOMA2-IR (1,8-12), and we have previously shown that several markers of iron metabolism were also cross-sectionally associated with an index of adipocyte IR (14). In the present study, longitudinal analysis of 7-year follow-up data with (re-)measurement of insulin at several time-points during the OGTT yielded three novel findings. First, we showed that, over a 7-year period, serum ferritin and transferrin at baseline were prospectively associated with HOMA2-IR, adipocyte IR, and a novel marker of hepatic $I R$, independently of classical risk factors and inflammatory markers. In addition, we showed that serum ferritin and transferrin were longitudinally associated with glucose tolerance, including $A \cup C_{\text {glucose }}$ during the OGTT. Third, we observed similar positive associations for serum iron and NTBI, although not statistically significant. Taken together, these results suggest that iron metabolism may be involved in the development of IR in several tissues, eventually leading to impaired glucose metabolism and T2DM.

Our findings agree with a previous prospective cohort study of 944 subjects, in which baseline ferritin and transferrin were associated with fasting insulin and HOMA2-IR at both baseline and 6-year follow-up $(15,16)$. We confirmed these associations, and, in addition, showed that these associations were independent of classical risk factors for IR, such as dietary energy intake, physical activity, smoking status, a positive family history of T2DM, obesity, or low-grade inflammation. We also extended these previous observations by showing significant associations of ferritin and transferrin not only with fasting glucose and insulin (and hence HOMA2-IR), but also with $2 \mathrm{~h}$ glucose and $\mathrm{AUC}_{\text {glucose }}$ during the OGTT. In agreement with these results, increases in body iron stores (measured by soluble transferrin receptor / ferritin ratio) in women from premenopause to postmenopause have recently been associated with increases in HOMA2-IR (32), while changes in transferrin have been related to changes in HOMA2-IR during a 1-year lifestyle intervention study (33).

Important novel findings of the present study are the independent associations of baseline ferritin and transferrin with hepatic and adipocyte IR over 7 years. Although 
calculations of HOMA2-IR, hepatic IR and adipocyte IR all included fasting insulin, each of these IR measures has been developed and validated with euglycaemic hyperinsulinaemic clamp studies that specifically investigated glucose disposal (for $80-90 \%$ accounted for by muscle) $(22,34)$, suppression of endogenous (hepatic) glucose production $(23,24)$, and suppression of NEFA turnover (adipocytes) (25-27), respectively. In addition, we have previously shown that associations with the adipocyte IR index were confirmed by associations with NEFAs independent of fasting insulin levels (14), corroborating the view that HOMA2-IR and adipocyte IR index do not represent the same site of IR.

Our epidemiological data are supported by published in vitro and in vivo observations. In vitro research has shown that incubation of Hep2G cells with iron decreased insulin receptor mRNA expression, glucokinase expression, and insulin binding to Hep2G cells (35). Similarly, incubation of rat adipocytes with iron or transferrin impaired insulin-mediated glucose uptake, and increased lipolysis $(36,37)$, and also led to decreased adiponectin mRNA and protein levels (38). Moreover, an iron-enriched diet in mice led to increased hepatic iron stores and visceral adipose tissue iron accumulation, accompanied by systemic and adipocyte IR in one study (39), as well as increased adipocyte iron stores (characterized by decrease in transferrin receptor mRNA levels) with lower adiponectin mRNA and protein levels, and lower glucose disposal in another study (38). In line with this, an iron-restricted diet or chelation therapy led to lower hepatic iron levels, lower HOMA-IR and $A \cup C_{\text {glucose, }}$ lower NEFAs and triglycerides, and higher serum adiponectin in mice $(38,40)$. Finally, recent studies in humans showed that hepatic iron content, measured by MRI, was associated with OGTT-derived measures of IR (13), and was higher in patients with prediabetes or T2DM compared with NGM (41). Altogether, these observations support the hypothesis that body iron stores and/or iron metabolism-related factors such as ferritin or transferrin may contribute to the development of IR in several tissues, including muscle, liver, and adipocytes.

In contrast with the extensive amount of literature on serum ferritin, data on the relation between serum iron or NTBI and IR are scarce. We and others have previously shown that NTBI concentrations were higher and more frequently detectable in patients with T2DM or the metabolic syndrome, and that serum iron and NTBI levels were cross-sectionally associated with adipocyte IR $(14,42)$. In the present study, serum iron and NTBI showed positive trends with IR and glucose metabolism over a 7-year period. A possible explanation for this could be that serum iron and NTBI are characterized by more random error (due to biological circadian fluctuations and day-to-day variations) than serum proteins such as ferritin and transferrin. Such random error may attenuate the magnitude of regression coefficients (attenuation bias) to such an extent that our study lacks sufficient power to detect significant associations. Notably, the strength of these associations did not decrease in any of the sensitivity analyses, and some associations of iron and NTBI reached statistical 
significance after excluding all subjects with transferrin saturation $>45 \%$, although the biological meaning of this is unclear.

The present observational cohort study generated a complete set of associations of five markers of iron metabolism with metabolic outcomes related to both IR and glucose tolerance at 7-year follow-up, adjusted for major (potential) confounders. A major limitation of the study is that we did not measure markers of iron metabolism at follow-up. Therefore, we were unable to investigate whether changes in iron markers were associated with changes in metabolic variables. Using only baseline measurements of iron metabolism, we did not observe significant associations between baseline ferritin and transferrin and changes in HOMA2-IR, in contrast with 2 previous studies $(15,16)$. However, our change analyses were carried out in only 386 subjects, and may therefore be underpowered to detect small within-subject effects, or may have been influenced by selective attrition (unhealthy subjects not attending follow-up), likely to have underestimated the associations. On the other hand, in these previous studies, change models were adjusted for baseline values of the outcome $(15,16)$, which may have led to inflation of the changes (within-subjects) coefficients (43). In addition, within-subject effects may be relatively small vs. between-subject effects in the present middle-aged population, because changes or differences in (markers of) iron metabolism may already have led to changes in IR before the age of 60 years. A second limitation of the present study is that the observed associations do not allow us to draw definite conclusions on causality. In a reverse causation-model, insulin may upregulate transferrin receptor expression, and hence transferrin and ferritin levels $(44,45)$. However, trial data have shown that iron depletion by phlebotomy decreases HOMA2-IR, improves glucose tolerance, and increases serum adiponectin $(38,46,47)$, supporting the current hypotheses. Whether this holds true for hepatic and adipocyte IR remains to be examined. Finally, our study consisted of middle-aged and older, Caucasian subjects who were selected on the basis of an increased risk for metabolic and cardiovascular disease, and extrapolation to other study populations or other ethnicities should be done with caution.

In conclusion, we have shown that baseline concentrations of both serum ferritin and transferrin were independently associated with indices of IR in muscle, liver, and adipocytes, and also with glucose tolerance over a 7-year follow-up period. These findings suggest that iron stores and/or iron metabolism-related factors such as ferritin or transferrin may contribute to the development of IR in several tissues, early in the pathogenesis of T2DM. Therefore, iron metabolism, and its effects on insulin sensitivity of adipose tissue, the liver, and muscle, constitutes a novel and interesting subject for future research, both in vitro and in vivo. 


\section{References}

1. Syrovatka P, Kraml P, Potockova J, Fialova L, Vejrazka M, Crkovska J, Andel M: Relationship between increased body iron stores, oxidative stress and insulin resistance in healthy men. Ann Nutr Metab 2009;54:268-274

2. Beard JL: Iron biology in immune function, muscle metabolism and neuronal functioning. J Nutr 2001; 131:568S-579S; discussion 580S

3. Houstis N, Rosen ED, Lander ES: Reactive oxygen species have a causal role in multiple forms of insulin resistance. Nature 2006;440:944-948

4. Pietrangelo A: Hereditary hemochromatosis--a new look at an old disease. N Engl J Med 2004;350: 2383-2397

5. Jiang R, Ma J, Ascherio A, Stampfer MJ, Willett WC, Hu FB: Dietary iron intake and blood donations in relation to risk of type 2 diabetes in men: a prospective cohort study. Am J Clin Nutr 2004;79:70-75

6. Lee DH, Folsom AR, Jacobs DR, Jr.: Dietary iron intake and Type 2 diabetes incidence in postmenopausal women: the lowa Women's Health Study. Diabetologia 2004;47:185-194

7. Luan de C, Li H, Li SJ, Zhao Z, Li X, Liu ZM: Body iron stores and dietary iron intake in relation to diabetes in adults in North China. Diabetes Care 2008;31:285-286

8. Haap M, Fritsche A, Mensing HJ, Haring HU, Stumvoll M: Association of high serum ferritin concentration with glucose intolerance and insulin resistance in healthy people. Ann Intern Med 2003; 139:869-871

9. Jehn M, Clark JM, Guallar E: Serum ferritin and risk of the metabolic syndrome in U.S. adults. Diabetes Care 2004;27:2422-2428

10. Lee BK, Kim Y, Kim YI: Association of serum ferritin with metabolic syndrome and diabetes mellitus in the South Korean general population according to the Korean National Health and Nutrition Examination Survey 2008. Metabolism 2011;60:1416-1424

11. Chen J, Wildman RP, Hamm LL, Muntner P, Reynolds K, Whelton PK, He J: Association between inflammation and insulin resistance in U.S. nondiabetic adults: results from the Third National Health and Nutrition Examination Survey. Diabetes Care 2004;27:2960-2965

12. Bozzini C, Girelli D, Olivieri O, Martinelli N, Bassi A, De Matteis G, Tenuti I, Lotto V, Friso S, Pizzolo F, Corrocher R: Prevalence of body iron excess in the metabolic syndrome. Diabetes Care 2005;28: 2061-2063

13. Haap M, Machann J, von Friedeburg C, Schick F, Stefan N, Schwenzer NF, Fritsche A, Haring HU, Thamer C: Insulin sensitivity and liver fat: role of iron load. J Clin Endocrinol Metab 2011;96:E958-961

14. Wlazlo N, van Greevenbroek MM, Ferreira I, Jansen EH, Feskens EJ, van der Kallen CJ, Schalkwijk CG, Bravenboer B, Stehouwer CD: Iron metabolism is associated with adipocyte insulin resistance and plasma adiponectin: the Cohort on Diabetes and Atherosclerosis Maastricht (CODAM) study. Diabetes Care 2013;36:309-315

15. Fumeron F, Pean F, Driss F, Balkau B, Tichet J, Marre M, Grandchamp B: Ferritin and transferrin are both predictive of the onset of hyperglycemia in men and women over 3 years: the data from an epidemiological study on the Insulin Resistance Syndrome (DESIR) study. Diabetes Care 2006;29: 2090-2094

16. Vari IS, Balkau B, Kettaneh A, Andre P, Tichet J, Fumeron F, Caces E, Marre M, Grandchamp B, Ducimetiere $P$ : Ferritin and transferrin are associated with metabolic syndrome abnormalities and their change over time in a general population: Data from an Epidemiological Study on the Insulin Resistance Syndrome (DESIR). Diabetes Care 2007;30:1795-1801

17. Wlazlo N, van Greevenbroek MM, Ferreira I, Jansen EJ, Feskens EJ, van der Kallen CJ, Schalkwijk CG, Bravenboer B, Stehouwer CD: Low-grade inflammation and insulin resistance independently explain substantial parts of the association between body fat and serum C3: The CODAM study. Metabolism 2012;61:1787-1796

18. Du $H$, van der AD, van Bakel MM, van der Kallen CJ, Blaak EE, van Greevenbroek MM, Jansen EH, Nijpels G, Stehouwer CD, Dekker JM, Feskens EJ: Glycemic index and glycemic load in relation to food and nutrient intake and metabolic risk factors in a Dutch population. Am J Clin Nutr 2008;87:655-661 
19. Swinkels DW, Fleming RE: Novel observations in hereditary hemochromatosis: potential implications for clinical strategies. Haematologica 2011;96:485-488

20. van Bussel BC, Ferreira I, van de Waarenburg MP, van Greevenbroek MM, van der Kallen CJ, Henry RM, Feskens EJ, Stehouwer CD, Schalkwijk CG: Multiple inflammatory biomarker detection in a prospective cohort study: a cross-validation between well-established single-biomarker techniques and an electrochemiluminescense-based multi-array platform. PLoS One 2013;8:e58576; DOI 10.1371/journal.pone.0058576

21. Martin RF: General deming regression for estimating systematic bias and its confidence interval in method-comparison studies. Clin Chem 2000;46:100-104

22. Wallace TM, Levy JC, Matthews DR: Use and abuse of HOMA modeling. Diabetes Care 2004;27: 1487-1495

23. Abdul-Ghani MA, Matsuda M, Balas B, DeFronzo RA: Muscle and liver insulin resistance indexes derived from the oral glucose tolerance test. Diabetes Care 2007;30:89-94

24. Vangipurapu J, Stancakova A, Kuulasmaa T, Paananen J, Kuusisto J, Ferrannini E, Laakso M: A novel surrogate index for hepatic insulin resistance. Diabetologia 2011;54:540-543

25. Vangipurapu J, Stancakova A, Pihlajamaki J, Kuulasmaa TM, Kuulasmaa T, Paananen J, Kuusisto J, Ferrannini E, Laakso M: Association of indices of liver and adipocyte insulin resistance with 19 confirmed susceptibility loci for type 2 diabetes in 6,733 non-diabetic Finnish men. Diabetologia 2011; 54:563-571

26. Abdul-Ghani MA, Molina-Carrion M, Jani R, Jenkinson C, Defronzo RA: Adipocytes in subjects with impaired fasting glucose and impaired glucose tolerance are resistant to the anti-lipolytic effect of insulin. Acta Diabetol 2008;45:147-150

27. Groop LC, Bonadonna RC, DelPrato S, Ratheiser K, Zyck K, Ferrannini E, DeFronzo RA: Glucose and free fatty acid metabolism in non-insulin-dependent diabetes mellitus. Evidence for multiple sites of insulin resistance. J Clin Invest 1989;84:205-213

28. Wendel-Vos GC, Schuit AJ, Saris WH, Kromhout D: Reproducibility and relative validity of the short questionnaire to assess health-enhancing physical activity. J Clin Epidemiol 2003;56:1163-1169

29. van Bussel BC, Henry RM, Schalkwijk CG, Ferreira I, Feskens EJ, Streppel MT, Smulders YM, Twisk JW, Stehouwer CD: Fish consumption in healthy adults is associated with decreased circulating biomarkers of endothelial dysfunction and inflammation during a 6-year follow-up. J Nutr 2011;141:1719-1725

30. Levey AS, Bosch JP, Lewis JB, Greene T, Rogers N, Roth D: A more accurate method to estimate glomerular filtration rate from serum creatinine: a new prediction equation. Modification of Diet in Renal Disease Study Group. Ann Intern Med 1999;130:461-470

31. Twisk JWR: Applied longitudinal data analysis for epidemiology: a practical guide. Cambridge, Cambridge University Press, 2003

32. Kim C, Nan B, Kong S, Harlow S: Changes in Iron Measures over Menopause and Associations with Insulin Resistance. J Womens Health (Larchmt) 2012;21:872-877

33. Roumen C, Feskens EJ, Jansen EH, Saris WH, Blaak EE: Changes in transferrin are related to changes in insulin resistance: the SLIM study. Diabet Med 2008;25:1478-1482

34. DeFronzo RA, Ferrannini E, Hendler R, Felig P, Wahren J: Regulation of splanchnic and peripheral glucose uptake by insulin and hyperglycemia in man. Diabetes 1983;32:35-45

35. Fargion S, Dongiovanni P, Guzzo A, Colombo S, Valenti L, Fracanzani AL: Iron and insulin resistance. Aliment Pharmacol Ther 2005;22 Suppl 2:61-63

36. Green A, Basile R, Rumberger JM: Transferrin and iron induce insulin resistance of glucose transport in adipocytes. Metabolism 2006;55:1042-1045

37. Rumberger JM, Peters T, Jr., Burrington C, Green A: Transferrin and iron contribute to the lipolytic effect of serum in isolated adipocytes. Diabetes 2004;53:2535-2541

38. Gabrielsen JS, Gao Y, Simcox JA, Huang J, Thorup D, Jones D, Cooksey RC, Gabrielsen D, Adams TD, Hunt SC, Hopkins PN, Cefalu WT, McClain DA: Adipocyte iron regulates adiponectin and insulin sensitivity. J Clin Invest 2012;122:3529-3540

39. Dongiovanni P, Ruscica M, Benedan L, Borroni V, Recalcati S, Steffani L, al. e: Dietary iron overload induces visceral adipose tissue insulin resistance associated with hyper-resistinemia, and synergizes with obesity and fatty liver in inducing systemic insulin resistance. J Hepatol 2011;54:S505 
40. Cooksey RC, Jones D, Gabrielsen S, Huang J, Simcox JA, Luo B, Soesanto Y, Rienhoff H, Abel ED, McClain DA: Dietary iron restriction or iron chelation protects from diabetes and loss of beta-cell function in the obese (ob/ob lep-/-) mouse. Am J Physiol Endocrinol Metab 2010;298:E1236-1243

41. Zheng $\mathrm{X}$, Jiang $\mathrm{T}, \mathrm{Wu} \mathrm{H}$, Zhu D, Wang L, Qi R, Li M, Ling C: Hepatic iron stores are increased as assessed by magnetic resonance imaging in a Chinese population with altered glucose homeostasis. Am J Clin Nutr 2011;94:1012-1019

42. Lee DH, Liu DY, Jacobs DR, Jr., Shin HR, Song K, Lee IK, Kim B, Hider RC: Common presence of nontransferrin-bound iron among patients with type 2 diabetes. Diabetes Care 2006;29:1090-1095

43. Glymour MM, Weuve J, Berkman LF, Kawachi I, Robins JM: When is baseline adjustment useful in analyses of change? An example with education and cognitive change. Am J Epidemiol 2005;162: 267-278

44. Davis RJ, Corvera S, Czech MP: Insulin stimulates cellular iron uptake and causes the redistribution of intracellular transferrin receptors to the plasma membrane. J Biol Chem 1986;261:8708-8711

45. O'Riordain MG, Ross JA, Fearon KC, Maingay J, Farouk M, Garden OJ, Carter DC: Insulin and counterregulatory hormones influence acute-phase protein production in human hepatocytes. Am J Physiol 1995;269:E323-330

46. Fernandez-Real JM, Penarroja G, Castro A, Garcia-Bragado F, Hernandez-Aguado I, Ricart W: Blood letting in high-ferritin type 2 diabetes: effects on insulin sensitivity and beta-cell function. Diabetes 2002;51:1000-1004

47. Valenti L, Fracanzani AL, Dongiovanni P, Bugianesi E, Marchesini G, Manzini P, Vanni E, Fargion S: Iron depletion by phlebotomy improves insulin resistance in patients with nonalcoholic fatty liver disease and hyperferritinemia: evidence from a case-control study. Am J Gastroenterol 2007;102:1251-1258 


\section{Supplementary tables}

Table S3.3.1 Associations of baseline transferrin saturation with insulin resistance over 7 years.

\begin{tabular}{|c|c|c|c|}
\hline Outcome & Model & $\beta$ & $\begin{array}{c}\text { Transferrin saturation } \\
\text { (per 1\%) } \\
95 \% \mathrm{Cl} \\
\end{array}$ \\
\hline HOMA2-IR & 1 & -0.78 & $-1.17 ;-0.39 \ddagger$ \\
\hline \multirow[t]{3}{*}{ (\% increase) } & 2 & -0.60 & $-0.96 ;-0.25 \dagger$ \\
\hline & 3 & -0.20 & $-0.50 ; 0.10$ \\
\hline & 4 & -0.15 & $-0.45 ; 0.15$ \\
\hline Hepatic IR & 1 & -0.53 & $-0.96 ;-0.09 *$ \\
\hline \multirow[t]{3}{*}{ (\% increase) } & 2 & -0.42 & $-0.86 ; 0.01$ \\
\hline & 3 & -0.08 & $-0.49 ; 0.32$ \\
\hline & 4 & -0.08 & $-0.49 ; 0.34$ \\
\hline Adipocyte IR & 1 & -0.97 & $-1.44 ;-0.49 \ddagger$ \\
\hline \multirow[t]{3}{*}{ (\% increase) } & 2 & -0.59 & $-1.02 ;-0.15^{\dagger}$ \\
\hline & 3 & -0.16 & $-0.55 ; 0.23$ \\
\hline & 4 & -0.09 & $-0.46 ; 0.28$ \\
\hline
\end{tabular}

Bs are unstandardized regression coefficients and represent the longitudinal associations between baseline transferrin saturation on the one hand, and HOMA2-IR, hepatic insulin resistance (IR), and adipocyte insulin resistance (IR) at both baseline and follow up on the other hand. $95 \% \mathrm{Cl}$ : $95 \%$ confidence interval. Model 1 : crude associations; Model 2: model 1 + adjusted for age, sex, follow-up time, glucose metabolism status, baseline cardiovascular disease, baseline estimated glomerular filtration rate, smoking status, alcohol consumption, dietary energy intake, physical activity, family history of T2DM, use of medication, and ferritin and transferrin adjusted for each other; Model 3: model $2+$ adjusted for waist circumference; Model 4: model 3 + adjusted for low-grade inflammation score. ${ }^{*} p<0.05 ; \uparrow p<0.01 ; \neq p<0.001$.

Table S3.3.2 Associations of baseline transferrin saturation with glucose tolerance over 7 years.

\begin{tabular}{|c|c|c|c|}
\hline Outcome & Model & $\beta$ & $\begin{array}{c}\text { Transferrin saturation } \\
\text { (per 1\%) } \\
95 \% \mathrm{Cl}\end{array}$ \\
\hline \multirow[t]{4}{*}{ Fasting glucose } & 1 & -0.12 & $-0.24 ; 0.01$ \\
\hline & 2 & -0.10 & $-0.18 ;-0.01 *$ \\
\hline & 3 & -0.04 & $-0.12 ; 0.04$ \\
\hline & 4 & -0.03 & $-0.11 ; 0.04$ \\
\hline \multirow[t]{4}{*}{$2 \mathrm{~h}$ glucose } & 1 & -0.24 & $-0.56 ; 0.08$ \\
\hline & 2 & -0.07 & $-0.31 ; 0.17$ \\
\hline & 3 & 0.02 & $-0.21 ; 0.25$ \\
\hline & 4 & 0.04 & $-0.19 ; 0.27$ \\
\hline \multirow[t]{4}{*}{ AUC glucose } & 1 & -0.16 & $-0.37 ; 0.06$ \\
\hline & 2 & -0.10 & $-0.27 ; 0.06$ \\
\hline & 3 & -0.02 & $-0.18 ; 0.14$ \\
\hline & 4 & -0.01 & $-0.17 ; 0.15$ \\
\hline
\end{tabular}

$\beta$ s are unstandardized regression coefficients and represent the longitudinal associations between baseline transferrin saturation on the one hand, and fasting glucose, $2 \mathrm{~h}$ glucose, and area under the curve (AUC) for glucose (during oral glucose tolerance test) at both baseline and follow up on the other hand. $95 \% \mathrm{Cl}$ : $95 \%$ confidence interval. Model 1: crude associations; Model 2: model 1 + adjusted for age, sex, follow-up time, baseline cardiovascular disease, baseline estimated glomerular filtration rate, smoking status, alcohol consumption, dietary energy intake, physical activity, family history of T2DM, use of medication, and ferritin and transferrin adjusted for each other; Model 3: model $2+$ adjusted for waist circumference; Model 4: model 3 + adjusted for low-grade inflammation score. ${ }^{*} p<0.05 ; \uparrow p<0.01 ; \neq p<0.001$. 


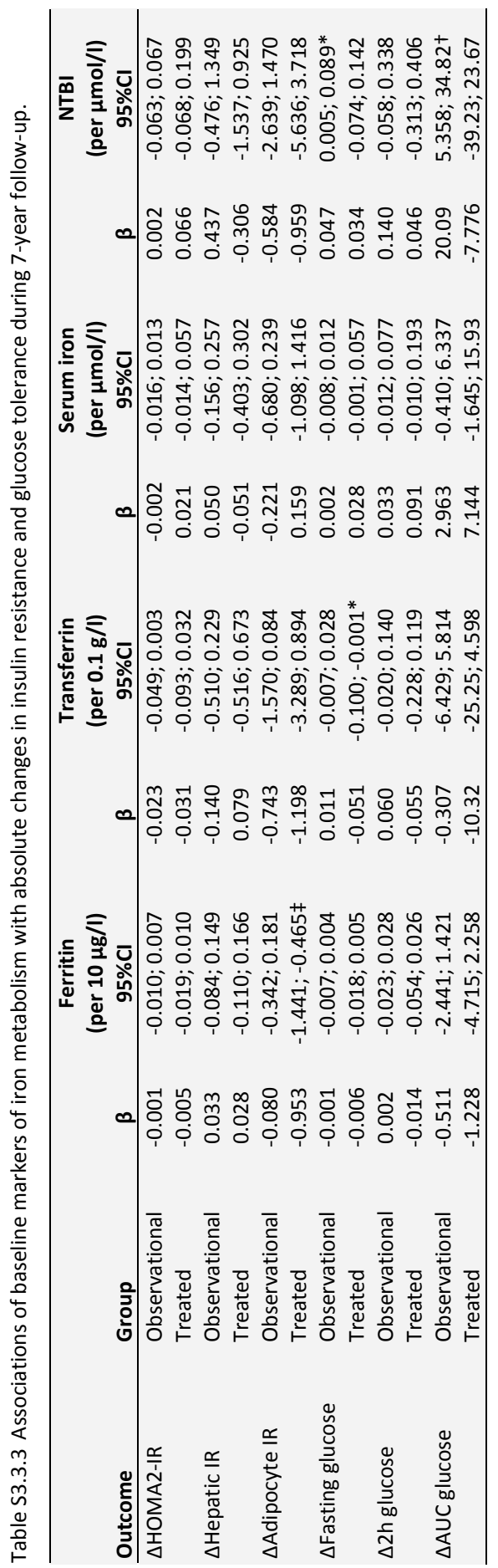

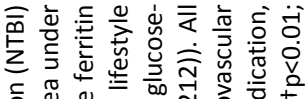

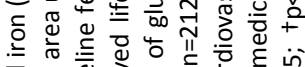

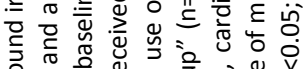

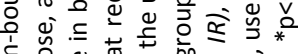

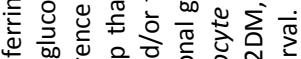
这 可

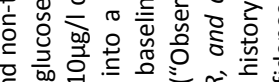
西

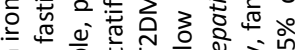

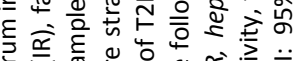

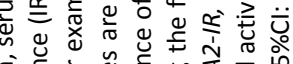

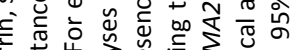

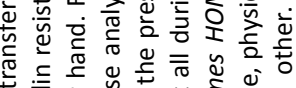
c)

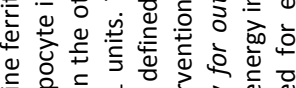

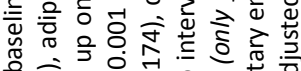

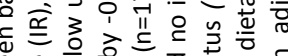
过品

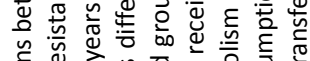

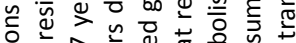

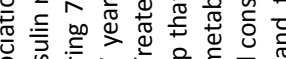

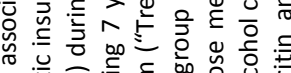

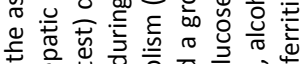

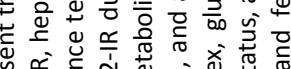

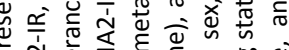
원

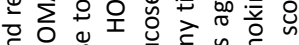

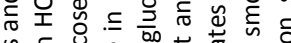

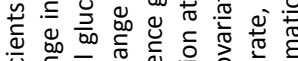
递 \&

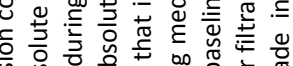

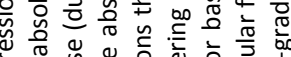

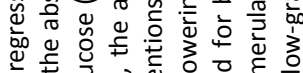

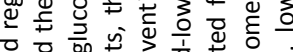

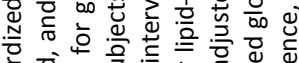

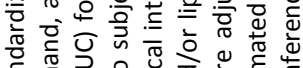

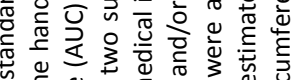

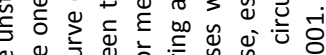
ه

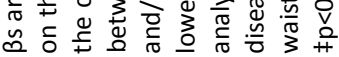




\section{Chapter 4

n

(n)

\section{The complement system \\ The complement system

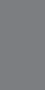

$x^{2}$ 


\section{Chapter 4.1}

The complement system in relation to the metabolic syndrome and type 2 diabetes mellitus 
Chapter 4.1 


\section{Introduction into the complement system}

The complement system is a large collection of proteins, both soluble in the circulation and membrane-bound, that is part of the innate immune system, and has important functions in the response to tissue injury and infection $(1,2)$. It is responsible for the defence against bacterial infections, bridging innate and adaptive immune responses, and for disposal of immune complexes and products of tissue injury. The complement system can be activated via three major pathways (classical, alternative, mannose-binding lectin) which converge on complement factor C3, leading to activation of the common terminal pathway. Upon terminal pathway activation, a cascade of subsequent protease activations results in cleavage of inactive complement proteins into large, membrane-bound fragments and small, soluble, anaphylatoxic fragments. The membrane-bound proteins together form terminal complement complexes (C5b-C9/TCC), which may, in sublytic amounts, have several effects on cell cycle and cell metabolism, including the induction of an inflammatory response through activation of the NLR family, notably the pyrin domain-containing 3 (NLRP3) inflammasome $(3,4)$. In higher amounts, lytic TCCs promote cytolysis by disrupting membrane integrity, and are therefore also known as membrane attack complexes (MAC). The anaphylatoxins of the complement system bind to their corresponding receptor and promote proinflammatory responses that lead to the attraction and activation of leucocytes to local inflammatory sites (5-7).

Complement factor 3 (C3) is the central component of the complement system, since activation through any of the three pathways results in cleavage of C3 into C3a and C3b (Figure 4.1.1). The membrane-bound C3b is essential for subsequent activation of the terminal complement pathway with concurrent formation of the important anaphylatoxin C5a and the complex of C5b-C9 (TCC/MAC). The anaphylatoxin C3a is a potent chemoattractant, and by binding to its receptor C3aR (8), it has been shown to increase the production of proinflammatory cytokines, such as interleukin-1 $\beta$ (IL-1 $\beta$ ), interleukin-6 (IL-6), and tumour necrosis factor- $\alpha$ (TNF- $\alpha$ ) (5-7). Plasma levels of C3a are, however, very low, as the C3a formed is rapidly desarginated to C3a-desarg by a carboxypeptidase. C3a-desarg has later been recognised as the lipogenic hormone acylation stimulating protein (ASP) (9). Although complement proteins are mainly produced by the liver (10), adipocytes have also been shown to produce and activate all factors of the alternative complement pathway, leading to generation of ASP, which, by acting on the $\mathrm{C} 5 \mathrm{a}$-like receptor 2 ( $\mathrm{C} 5 \mathrm{~L} 2)$, promotes the storage of glucose and lipids in adipocytes in a way similar to insulin (Figure 4.1.1) (11-14). 


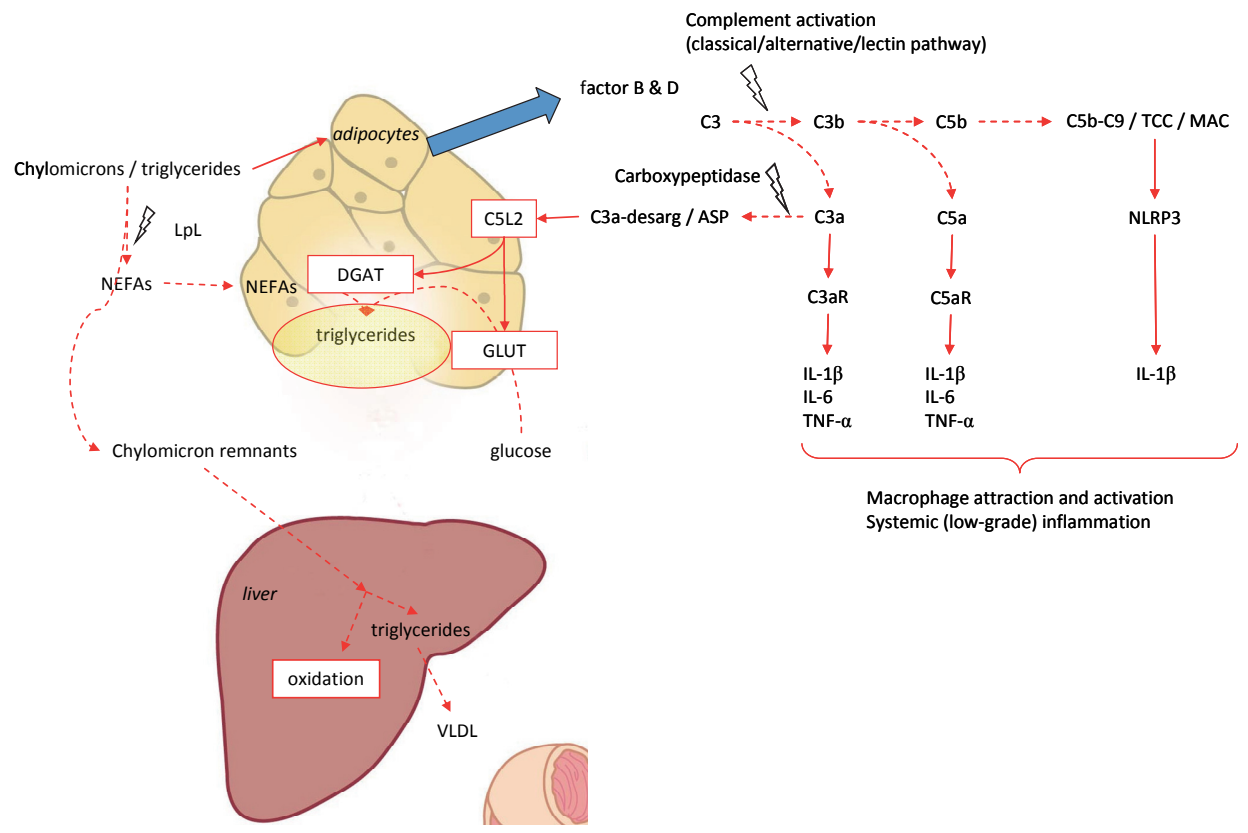

Figure 4.1.1 Role of the complement system in inflammation and metabolism

Throughout the human body, complement activation through any of the three pathways results in cleavage of $\mathrm{C} 3$ into $\mathrm{C} 3 \mathrm{a}$ and $\mathrm{C} 3 \mathrm{~b}$. C3b initiates the terminal complement activation cascade, which leads to cleavage of $\mathrm{C} 5$, and eventually to the formation of complexes $\mathrm{C5}$ b-C9, also called terminal complement complexes (TCCs). The anaphylatoxins C3a and C5a, by binding to their receptors, increase the production of proinflammatory cytokines interleukin-1 $\beta$ (IL-1B), interleukin-6 (IL-6), and tumour necrosis factor- $\alpha$ (TNF- $\alpha$ ), and promote proinflammatory responses that lead to the attraction and activation of more leucocytes to local inflammatory sites. TCCs, in sublytic amounts, induce an inflammatory response through the NLRP3 inflammasome, and, in higher amounts, form cytolytic membrane attack complexes (MAC).

In addition to the inflammatory component, the complement system is also involved in adipose tissue metabolism. Postprandial triglycerides are converted to glycerol and non-esterified fatty acids (NEFAs) by endothelial lipoprotein lipase (LPL), after which NEFAs can be taken up by adipocytes. In response to exposure to triglyceride-containing chylomicrons, adipocytes produce and secrete $C 3$ and factors $B$ and $D$, which lead to activation of the alternative pathway of complement activation. The anaphylatoxin C3a thus generated is rapidly desarginated by carboxypeptidases to C3a-desarg, also known as acylation-stimulating protein (ASP). After binding to the $\mathrm{C} 5 \mathrm{a}$-like receptor 2 (C5L2) on adipocytes, ASP increases glucose transporters (GLUT) and stimulates diglyceride acyltransferase (DGAT), thereby promoting the incorporation of glucose and NEFAs into triglycerides in adipocytes. The remaining triglycerides and NEFAs are diverted to the liver, where they are either stored, oxidized, or processed into very-low-densitylipoproteins (VLDL). The solid arrows denote direct effects, the dotted arrows denote conversion or transport, and the lightning bolts indicate enzymatic reactions. 


\section{The complement system in relation to obesity and disorders in glucose metabolism}

Over the past ten years, the complement system, and more specifically its central component $\mathrm{C} 3$, has increasingly been recognised as an emerging aetiological factor in the pathogenesis of metabolic diseases, such as the metabolic syndrome and type 2 diabetes mellitus (T2DM). As described in the general introduction of this thesis, local and/or systemic low-grade inflammation (LGI) is believed to play a major role in the development of (obesity-related) insulin resistance in several organs (15-20). The complement system, as part of both the innate and adaptive immune system, represents an important part of this inflammatory response. Plasma C3 levels, as the central component of the complement system, may at least be regarded an important biomarker of (obesity-related) LGI, since several proinflammatory cytokines (IL-1 $\alpha$, IL-1 $\beta$, IL-6, TNF- $\alpha$ and interferon- $\gamma$ (IFN- $\gamma$ )) have been shown to increase C3 expression and/or production in vitro in many cell types, including adipocytes and hepatocytes (21-23). In addition to being a biomarker, the complement system may also actively contribute to the development of insulin resistance and T2DM, by mechanisms as presented in the previous section, via which complement activation may perpetuate the ongoing local and/or systemic inflammation. Higher plasma C3 levels may be associated with more complement activation, resulting in higher levels of C3a as well as more distal activation products C5a and TCC. The corresponding receptors for these anaphylatoxins, $\mathrm{C} 3 \mathrm{aR}$ and $\mathrm{C} 5 \mathrm{aR}$, are highly expressed in adipose tissue and on Kupffer cells in the liver (24-27). As such, stimulation of these receptors may increase the production and secretion of several proinflammatory cytokines that lead to the attraction and activation of leucocytes in adipose tissue and liver (local inflammation), but after release into the circulation, also amplify the systemic inflammatory response (2). In agreement, blocking these anaphylatoxic pathways in mice models by knockout of the $\mathrm{C} 3 \mathrm{aR}$ or $\mathrm{C} 5 \mathrm{aR}$ led to decreased adipocyte size, less adipose tissue infiltration by macrophages, a reduction in tissue and plasma pro-inflammatory cytokines, and less systemic insulin resistance $(27,28)$. Moreover, inhibition of $\mathrm{C} 3 \mathrm{a}$ and $\mathrm{C} 5 \mathrm{a}$ signalling by blocking their receptors appeared to induce similar effects (26).

In addition to the role of the complement system in the inflammatory response, dysfunction of the C3-ASP-C5L2 system in adipocytes (ASP resistance) has also been put forward as an aetiological factor in the development of insulin resistance and T2DM (13). Data from mouse models have shown that inhibition of the lipogenic effects of the ASP-C5L2 axis by C5L2 knockout or anti-C5L2 antibodies led to decreased triglyceride storage in adipose tissue and delayed lipid clearance, but also to impaired glucose tolerance and (peripheral) insulin resistance $(29,30)$. In vitro research has shown that fatty acids and the pro-inflammatory cytokine, TNF- $\alpha$, are able to interfere with ASP-C5L2 signalling in human adipocytes $(31,32)$, suggesting that reduced ASP signalling 
may alter human adipocyte metabolism as well. In human subjects, fasting plasma C3 levels are correlated with postprandial triglyceride levels $(33,34)$, and it has been suggested that these fasting C3 levels are indicative of ASP resistance in the same way that fasting insulin levels are indicative of insulin resistance $(13,32,33)$, although this has been disputed in the face of contradictory results from in vitro and animal studies (35).

Human (epidemiological) data on the role of C3 in relation to metabolic disorders are usually limited to cross-sectional studies in which adipose tissue $\mathrm{C} 3$ expression and systemic C3 concentrations have consistently and strongly been linked to several measures of body fat (36-40). Systemic C3 levels decrease with weight loss and increase with weight gain $(41,42)$. Besides a close relation with adiposity measures, plasma C3 levels have also been cross-sectionally associated with insulin resistance (as measured by homeostasis model assessment (HOMA-IR) or hyperinsulinaemic euglycaemic clamp studies), the dyslipidaemia of the metabolic syndrome, and with type 2 diabetes mellitus (T2DM) $(34,36,37,43)$. Recent longitudinal data from only two studies have shown associations of $\mathrm{C} 3$ with the development of obesity and the metabolic syndrome $(44,45)$, and also with incident type 2 diabetes mellitus (46). However, this latter cohort consisted of only adult men $(45,46)$, and there are, to our knowledge, no longitudinal data on the relation between (changes in) C3 levels and (changes in) insulin resistance.

In summary, there is substantial evidence for a contribution of $\mathrm{C} 3$, at least partly via C3 activation, in the development of insulin resistance, the metabolic syndrome, and T2DM. However, longitudinal data on this subject are still scarce, and the causal relationships and order of the events that occur in the vicious cycles of complement production/activation, inflammation, and insulin resistance, still remain to be unravelled.

\section{The complement system and fatty liver disease}

There is growing evidence that chronic inflammation and activation of the immune system also contribute to the development and progression of alcoholic and nonalcoholic fatty liver disease (AFLD and NAFLD), with a key role for the cytokine TNF- $\alpha$ produced by Kupffer cells $(47,48)$. As described above, the C3aR and C5aR are highly expressed on Kupffer cells in the liver $(24,25,27)$, and by acting on these receptors, C3a and C5a have been shown to stimulate prostaglandin, TNF- $\alpha$, and IL- 6 secretion by Kupffer cells $(24,49,50)$. Therefore, activation of the complement system with generation of these anaphylatoxins has been implicated in the pathogenesis of AFLD and NAFLD.

Several animal models of AFLD have demonstrated increased hepatic deposition of complement factors C1, C3 and C8 (51-53). Moreover, C3 knockout mice were protected from alcohol-induced liver steatosis and hepatocyte injury $(54,55)$. Finally, 
mice missing either the C3aR or C5aR (by knockout) or mice that were depleted of Kupffer cells all lacked the alcohol-induced increases in hepatic TNF- $\alpha$ (52), suggesting that $\mathrm{C} 3 \mathrm{a}$ and $\mathrm{C} 5 \mathrm{a}$ signalling in Kupffer cells may contribute to TNF- $\alpha$ production and subsequent liver damage in AFLD. For NAFLD, there is also some evidence for a role of complement activation. In a C3aR knockout mouse model given a high fat diet, liver triglycerides and plasma liver enzymes were lower compared with wild-type mice (27).

Human data on the role of the complement system in AFLD and NAFLD are very scarce. To our knowledge, no studies have been published on the role of complement activation in human AFLD. A case series of morbidly obese humans with non-alcoholic steatohepatitis (NASH) showed abundant activated C3b deposition in liver biopsies, but not in healthy controls without steatosis (56). In addition, plasma levels of C3a were higher in a small sample of patients with NAFLD than in healthy controls (57).

\section{Outline and aims of this chapter}

Accumulating evidence from animal studies and in vitro research has shown that the complement system is intertwined with various features of the metabolic syndrome, ranging from obesity, low-grade inflammation, and insulin resistance, to T2DM, and fatty liver disease (AFLD and NAFLD). Although the evidence is suggestive, the translation of these findings to human populations calls for more epidemiological data, with systematic measurement of both adiposity measures, inflammatory markers, and markers of insulin resistance. For that reason, we explored several aspects of the complement system in relation to obesity, insulin resistance, T2DM, and fatty liver disease within the CODAM study (Cohort on Diabetes and Atherosclerosis Maastricht).

In the first study, we cross-sectionally explored metabolic pathways that underlie the obesity-related differences in circulating C3 levels (Chapter 4.2). Since low-grade inflammation and insulin resistance are common features of obesity, and are also related to circulating C3 levels, we investigated, by means of multiple mediation analyses, whether they constitute mechanisms by which body fat is related to circulating C3 levels.

In the second study, we extensively analysed the relation of plasma C3 levels with insulin resistance and glucose tolerance in a longitudinal study with 7-year follow-up data (Chapter 4.3). We investigated the longitudinal associations between (changes in) C3 levels with (changes in) whole body (HOMA2-IR) and organ-specific (liver, adipocyte) insulin resistance and measures of glucose tolerance during 7-year follow-up. Moreover, we examined whether plasma C3 levels at baseline were associated with incident T2DM in a population of both men and women.

In the third study, we explored the role of complement activation in human AFLD and NAFLD, by investigating whether plasma C3a levels, as a marker of complement activation by any of the three pathways, were cross-sectionally associated with hepatic 
steatosis and markers of hepatocellular injury (Chapter 4.4). Given the potentially important role of complement-induced TNF- $\alpha$ production in these liver diseases, we also examined the mediating role of (systemic) TNF- $\alpha$ levels in these associations. Finally, we investigated in a fourth study whether plasma levels of C5a and SC5-C9, which indicate distal complement activation up to TCC/MAC formation, were crosssectionally associated with hepatic steatosis and hepatocellular injury (Chapter 4.5). 


\section{References}

1. Walport MJ: Complement. First of two parts. N Engl J Med 2001;344:1058-1066

2. Markiewski MM, Lambris JD: The role of complement in inflammatory diseases from behind the scenes into the spotlight. Am J Pathol 2007;171:715-727

3. Triantafilou K, Hughes TR, Triantafilou M, Morgan BP: The complement membrane attack complex triggers intracellular Ca2+ fluxes leading to NLRP3 inflammasome activation. J Cell Sci 2013;126: 2903-2913

4. Laudisi F, Spreafico R, Evrard M, Hughes TR, Mandriani B, Kandasamy M, Morgan BP, Sivasankar B, Mortellaro A: Cutting Edge: The NLRP3 Inflammasome Links Complement-Mediated Inflammation and IL-1beta Release. J Immunol 2013;191:1006-1010

5. Takabayashi T, Vannier E, Clark BD, Margolis NH, Dinarello CA, Burke JF, Gelfand JA: A new biologic role for C3a and C3a desArg: regulation of TNF-alpha and IL-1 beta synthesis. J Immunol 1996;156: 3455-3460

6. Fischer WH, Jagels MA, Hugli TE: Regulation of IL-6 synthesis in human peripheral blood mononuclear cells by C3a and C3a(desArg). J Immunol 1999;162:453-459

7. Asgari E, Le Friec G, Yamamoto H, Perucha E, Sacks SS, Kohl J, Cook HT, Kemper C: C3a modulates IL1beta secretion in human monocytes by regulating ATP efflux and subsequent NLRP3 inflammasome activation. Blood 2013; DOI 10.1182/blood-2013-05-502229

8. Klos A, Bank S, Gietz C, Bautsch W, Kohl J, Burg M, Kretzschmar T: C3a receptor on dibutyryl-cAMPdifferentiated U937 cells and human neutrophils: the human C3a receptor characterized by functional responses and 125I-C3a binding. Biochemistry 1992;31:11274-11282

9. Baldo A, Sniderman AD, St-Luce S, Avramoglu RK, Maslowska M, Hoang B, Monge JC, Bell A, Mulay S, Cianflone K: The adipsin-acylation stimulating protein system and regulation of intracellular triglyceride synthesis. J Clin Invest 1993;92:1543-1547

10. Alper CA, Johnson AM, Birtch AG, Moore FD: Human C'3: evidence for the liver as the primary site of synthesis. Science 1969;163:286-288

11. Saleh J, Summers LK, Cianflone K, Fielding BA, Sniderman AD, Frayn KN: Coordinated release of acylation stimulating protein (ASP) and triacylglycerol clearance by human adipose tissue in vivo in the postprandial period. J Lipid Res 1998;39:884-891

12. Germinario R, Sniderman AD, Manuel S, Lefebvre SP, Baldo A, Cianflone K: Coordinate regulation of triacylglycerol synthesis and glucose transport by acylation-stimulating protein. Metabolism 1993;42:574-580

13. Cianflone K, Xia Z, Chen LY: Critical review of acylation-stimulating protein physiology in humans and rodents. Biochim Biophys Acta 2003;1609:127-143

14. Kalant D, MacLaren R, Cui W, Samanta R, Monk PN, Laporte SA, Cianflone K: C5L2 is a functional receptor for acylation-stimulating protein. J Biol Chem 2005;280:23936-23944

15. Xu H, Barnes GT, Yang Q, Tan G, Yang D, Chou CJ, Sole J, Nichols A, Ross JS, Tartaglia LA, Chen H: Chronic inflammation in fat plays a crucial role in the development of obesity-related insulin resistance. J Clin Invest 2003;112:1821-1830

16. Chen J, Wildman RP, Hamm LL, Muntner P, Reynolds K, Whelton PK, He J: Association between inflammation and insulin resistance in U.S. nondiabetic adults: results from the Third National Health and Nutrition Examination Survey. Diabetes Care 2004;27:2960-2965

17. van Greevenbroek MM, Schalkwijk CG, Stehouwer CD: Obesity-associated low-grade inflammation in type 2 diabetes mellitus: causes and consequences. Neth J Med 2013;71:174-187

18. Krogh-Madsen R, Plomgaard P, Moller K, Mittendorfer B, Pedersen BK: Influence of TNF-alpha and IL-6 infusions on insulin sensitivity and expression of IL-18 in humans. Am J Physiol Endocrinol Metab 2006;291:E108-114

19. Yudkin JS, Stehouwer CD, Emeis JJ, Coppack SW: C-reactive protein in healthy subjects: associations with obesity, insulin resistance, and endothelial dysfunction: a potential role for cytokines originating from adipose tissue? Arterioscler Thromb Vasc Biol 1999;19:972-978

20. Trayhurn P, Wood IS: Adipokines: inflammation and the pleiotropic role of white adipose tissue. $\mathrm{Br} \mathrm{J}$ Nutr 2004;92:347-355 
21. Volanakis JE: Transcriptional regulation of complement genes. Annu Rev Immunol 1995;13:277-305

22. Kalant D, Maslowska M, Scantlebury T, Wang H, Cianflone K: Control of lipogenesis in adipose tissue and the role of acylation stimulating protein. Can J Diab 2003;27:154-171

23. Peake PW, O'Grady S, Pussell BA, Charlesworth JA: Detection and quantification of the control proteins of the alternative pathway of complement in 3T3-L1 adipocytes. Eur J Clin Invest 1997;27:922-927

24. Strey CW, Markiewski M, Mastellos D, Tudoran R, Spruce LA, Greenbaum LE, Lambris JD: The proinflammatory mediators C3a and C5a are essential for liver regeneration. J Exp Med 2003;198:913923

25. Schlaf G, Schieferdecker HL, Rothermel E, Jungermann K, Gotze O: Differential expression of the C5a receptor on the main cell types of rat liver as demonstrated with a novel monoclonal antibody and by C5a anaphylatoxin-induced Ca2+ release. Lab Invest 1999;79:1287-1297

26. Lim J, lyer A, Suen JY, Seow V, Reid RC, Brown L, Fairlie DP: C5aR and C3aR antagonists each inhibit dietinduced obesity, metabolic dysfunction, and adipocyte and macrophage signaling. FASEB J 2013;27: 822-831

27. Mamane Y, Chung Chan C, Lavallee G, Morin N, Xu L, Huang J, Gordon R, Thomas W, Lamb J, Schadt EE, Kennedy BP, Mancini JA: The C3a anaphylatoxin receptor is a key mediator of insulin resistance and functions by modulating adipose tissue macrophage infiltration and activation. Diabetes 2009;58:20062017

28. Phieler J, Chung KJ, Chatzigeorgiou A, Klotzsche-von Ameln A, Garcia-Martin R, Sprott D, Moisidou M, Tzanavari T, Ludwig B, Baraban E, Ehrhart-Bornstein M, Bornstein SR, Mziaut H, Solimena M, Karalis KP, Economopoulou M, Lambris JD, Chavakis T: The Complement Anaphylatoxin C5a Receptor Contributes to Obese Adipose Tissue Inflammation and Insulin Resistance. J Immunol 2013; DOI: 10.4049/jimmunol.1300038

29. Cui W, Paglialunga S, Kalant D, Lu H, Roy C, Laplante M, Deshaies Y, Cianflone K: Acylation-stimulating protein/C5L2-neutralizing antibodies alter triglyceride metabolism in vitro and in vivo. Am J Physiol Endocrinol Metab 2007;293:E1482-1491

30. Paglialunga S, Schrauwen P, Roy C, Moonen-Kornips E, Lu H, Hesselink MK, Deshaies Y, Richard D, Cianflone K: Reduced adipose tissue triglyceride synthesis and increased muscle fatty acid oxidation in C5L2 knockout mice. J Endocrinol 2007;194:293-304

31. MacLaren R, Kalant $D$, Cianflone $K$ : The ASP receptor C5L2 is regulated by metabolic hormones associated with insulin resistance. Biochem Cell Biol 2007;85:11-21

32. Wen Y, Wang H, MacLaren R, Wu J, Lu H, Cianflone K: Palmitate and oleate induction of acylation stimulating protein resistance in 3T3-L1 adipocytes and preadipocytes. J Cell Biochem 2008;104: 391-401

33. Halkes CJ, van Dijk H, de Jaegere PP, Plokker HW, van Der Helm Y, Erkelens DW, Castro Cabezas M: Postprandial increase of complement component 3 in normolipidemic patients with coronary artery disease: effects of expanded-dose simvastatin. Arterioscler Thromb Vasc Biol 2001;21:1526-1530

34. van Oostrom AJ, Alipour A, Plokker TW, Sniderman AD, Cabezas MC: The metabolic syndrome in relation to complement component 3 and postprandial lipemia in patients from an outpatient lipid clinic and healthy volunteers. Atherosclerosis 2007;190:167-173

35. Klos A, Wende E, Wareham KJ, Monk PN: International Union of Pharmacology. LXXXVII. Complement peptide C5a, C4a, and C3a receptors. Pharmacol Rev 2013;65:500-543

36. Weyer C, Tataranni PA, Pratley RE: Insulin action and insulinemia are closely related to the fasting complement C3, but not acylation stimulating protein concentration. Diabetes Care 2000;23:779-785

37. Hernandez-Mijares A, Jarabo-Bueno MM, Lopez-Ruiz A, Sola-Izquierdo E, Morillas-Arino C, MartinezTriguero ML: Levels of $\mathrm{C} 3$ in patients with severe, morbid and extreme obesity: its relationship to insulin resistance and different cardiovascular risk factors. Int J Obes (Lond) 2007;31:927-932

38. Warnberg J, Nova E, Moreno LA, Romeo J, Mesana MI, Ruiz JR, Ortega FB, Sjostrom M, Bueno M, Marcos A: Inflammatory proteins are related to total and abdominal adiposity in a healthy adolescent population: the AVENA Study. Am J Clin Nutr 2006;84:505-512

39. Koistinen HA, Vidal H, Karonen SL, Dusserre E, Vallier P, Koivisto VA, Ebeling P: Plasma acylation stimulating protein concentration and subcutaneous adipose tissue C3 mRNA expression in nondiabetic and type 2 diabetic men. Arterioscler Thromb Vasc Biol 2001;21:1034-1039 
40. Gabrielsson BG, Johansson JM, Lonn M, Jernas M, Olbers T, Peltonen M, Larsson I, Lonn L, Sjostrom L, Carlsson B, Carlsson LM: High expression of complement components in omental adipose tissue in obese men. Obes Res 2003;11:699-708

41. Pomeroy C, Mitchell J, Eckert E, Raymond N, Crosby R, Dalmasso AP: Effect of body weight and caloric restriction on serum complement proteins, including Factor D/adipsin: studies in anorexia nervosa and obesity. Clin Exp Immunol 1997; 108:507-515

42. H ernandez-Mijares A, Banuls C, Bellod L, Jover A, Sola E, Morillas C, Victor VM, Rocha M: Effect of weight loss on C3 and C4 components of complement in obese patients. Eur J Clin Invest 2012;42:503-509

43. Muscari A, Antonelli S, Bianchi G, Cavrini G, Dapporto S, Ligabue A, Ludovico C, Magalotti D, Poggiopollini G, Zoli M: Serum C3 is a stronger inflammatory marker of insulin resistance than Creactive protein, leukocyte count, and erythrocyte sedimentation rate: comparison study in an elderly population. Diabetes Care 2007;30:2362-2368

44. Onat A, Hergenc G, Can G, Kaya Z, Yuksel H: Serum complement C3: a determinant of cardiometabolic risk, additive to the metabolic syndrome, in middle-aged population. Metabolism 2010;59:628-634

45. Engstrom G, Hedblad B, Janzon L, Lindgarde F: Weight gain in relation to plasma levels of complement factor 3: results from a population-based cohort study. Diabetologia 2005;48:2525-2531

46. Engstrom G, Hedblad B, Eriksson KF, Janzon L, Lindgarde F: Complement C3 is a risk factor for the development of diabetes: a population-based cohort study. Diabetes 2005;54:570-575

47. Tilg H, Diehl AM: Cytokines in alcoholic and nonalcoholic steatohepatitis. N Engl J Med 2000;343: 1467-1476

48. Lucey MR, Mathurin P, Morgan TR: Alcoholic hepatitis. N Engl J Med 2009;360:2758-2769

49. Puschel GP, Hespeling $U$, Oppermann $M$, Dieter $P$ : Increase in prostanoid formation in rat liver macrophages (Kupffer cells) by human anaphylatoxin C3a. Hepatology 1993;18:1516-1521

50. Schieferdecker HL, Schlaf G, Jungermann K, Gotze O: Functions of anaphylatoxin C5a in rat liver: direct and indirect actions on nonparenchymal and parenchymal cells. Int Immunopharmacol 2001;1:469-481

51. Jarvelainen HA, Vakeva A, Lindros KO, Meri S: Activation of complement components and reduced regulator expression in alcohol-induced liver injury in the rat. Clin Immunol 2002;105:57-63

52. Roychowdhury S, McMullen MR, Pritchard MT, Hise AG, van Rooijen N, Medof ME, Stavitsky AB, Nagy LE: An early complement-dependent and TLR-4-independent phase in the pathogenesis of ethanolinduced liver injury in mice. Hepatology 2009;49:1326-1334

53. Cohen JI, Roychowdhury S, McMullen MR, Stavitsky AB, Nagy LE: Complement and alcoholic liver disease: role of $\mathrm{C} 1 \mathrm{q}$ in the pathogenesis of ethanol-induced liver injury in mice. Gastroenterology 2010; 139:664-674, 674 e661

54. Bykov I, Junnikkala S, Pekna M, Lindros KO, Meri S: Complement C3 contributes to ethanol-induced liver steatosis in mice. Ann Med 2006;38:280-286

55. Pritchard MT, McMullen MR, Stavitsky AB, Cohen JI, Lin F, Medof ME, Nagy LE: Differential contributions of $\mathrm{C} 3, \mathrm{C} 5$, and decay-accelerating factor to ethanol-induced fatty liver in mice. Gastroenterology 2007;132:1117-1126

56. Rensen SS, Slaats Y, Driessen A, Peutz-Kootstra CJ, Nijhuis J, Steffensen R, Greve JW, Buurman WA: Activation of the complement system in human nonalcoholic fatty liver disease. Hepatology 2009;50:1809-1817

57. Yesilova Z, Ozata M, Oktenli C, Bagci S, Ozcan A, Sanisoglu SY, Uygun A, Yaman H, Karaeren N, Dagalp K: Increased acylation stimulating protein concentrations in nonalcoholic fatty liver disease are associated with insulin resistance. Am J Gastroenterol 2005;100:842-849 


\section{Chapter 4

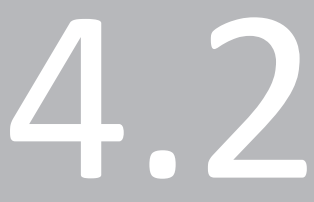

Low-grade inflammation and insulin resistance independently explain substantial parts of the association between body fat and serum C3

Nick Wlazlo, Marleen MJ van Greevenbroek, Isabel Ferreira, Eugene JHM Jansen, Edith JM Feskens, Carla JH van der Kallen, Casper G Schalkwijk, Bert Bravenboer, Coen DA Stehouwer 


\section{Abstract}

\section{Objective}

To investigate the role of low-grade inflammation and insulin resistance (HOMA2-IR) in adiposity-related increases in serum complement factor 3 (C3). Although C3 has been linked to type 2 diabetes and cardiovascular diseases, and C3 levels are closely related to body fat, the underlying mechanisms explaining this association are still unknown.

\section{Methods}

Adiposity measures (including BMI, waist circumference (WC), sagittal diameter and several skinfolds), HOMA2-IR and markers of inflammation (hs-CRP, IL-6, SAA, haptoglobin, ceruloplasmin, sICAM-1) were determined in 532 individuals (62\% men, mean age $59 \pm 6.9 \mathrm{yrs}$ ) from the Cohort on Diabetes and Atherosclerosis Maastricht study. Markers of inflammation were standardized and compiled into an averaged inflammation score. Cross-sectional associations between adiposity measures and C3 and the mediating role of low-grade inflammation and/or HOMA2-IR herein were analysed with multiple linear regression models.

\section{Results}

Adiposity measurements were significantly associated with $\mathrm{C} 3$ levels, with the strongest (adjusted) associations found for WC $(\beta=0.383 ; 95 \% \mathrm{Cl} 0.302-0.464)$ and sagittal diameter $(\beta=0.412 ; 95 \% \mathrm{Cl} 0.333-0.490)$. Further adjustment for inflammation and HOMA2-IR attenuated these associations to $\beta=0.115(95 \% \mathrm{Cl} 0.030-0.200)$ and $\beta=0.163(95 \% \mathrm{Cl} 0.082-0.244)$ respectively. Multiple mediation analyses showed that inflammation $[\beta=0.090(95 \% \mathrm{Cl} 0.060-0.126)]$ and HOMA2-IR $[\beta=0.179(95 \% \mathrm{Cl} 0.128-0.236)]$ each explained, independently of one another, a significant portion of the association between WC and C3 (23\% and 47\%, respectively). Similar mediation by inflammation (19-27\%) and HOMA2-IR (37-56\%) was found for other adiposity measures.

\section{Conclusion}

Systemic low-grade inflammation and insulin resistance may represent two independent pathways by which body fat leads to elevated C3 levels. 


\section{Introduction}

Serum complement factor 3 (C3) is an emerging risk factor in obesity-related metabolic and cardiovascular diseases (CVD). It has been associated with incident type 2 diabetes mellitus, myocardial infarction, and hypertension (1-4). Serum C3 levels are higher in subjects with insulin resistance or the metabolic syndrome than in healthy individuals, and are closely related to body fat (5-7). Although C3 is an established cardiometabolic risk factor, little attention has been paid to the metabolic pathways that underlie these obesity-related differences in serum C3.

Complement C3 is mainly produced by the liver, but other sources have been identified as well (8). Of note, estimations of the contribution of the liver to systemic C3 levels are primarily based on data from subjects who received a liver transplantation. Metabolically, such individuals cannot be compared to overweight or obese subjects that are generally included in studies on the association between C3 and cardiometabolic disease. Thus, the (relative) contribution of different sites of C3 production in these subjects remains to be conclusively established. It is plausible that at least a part of the observed differences in serum C3 originate in adipocyte and/or adipose tissue C3 production. Indeed, adipocytes have been shown to produce all components of the alternative complement pathway including C3 (9). The relative expression of C3 in adipocytes or adipose tissue is $10-20 \%$ of that seen in liver (10), and C3 mRNA expression is increased in adipose tissue of obese compared with lean subjects (11). In addition, systemic C3 concentrations are associated with several measures of body fat, including BMI, waist circumference (WC), hip circumference (HC), waist-to-hip-ratio (WHR), percentage body fat, sum of skinfolds, and visceral and subcutaneous adipose tissue areas on computed tomography $(5,10,12)$. Furthermore, C3 levels decrease with weight loss and increase with weight gain (13).

Although the association of body fat with $\mathrm{C} 3$ is clear, the mechanisms that underlie higher levels of serum C3 in obesity are still unknown. Obesity is characterized by elevated serum levels of several proinflammatory cytokines and proteins, reflecting a state of chronic, low-grade inflammation that is often accompanied by insulin resistance (14-16). Pro-inflammatory cytokines such as tumour necrosis factor- $\alpha$ (TNF- $\alpha$ ), interleukin-1 (IL-1), interleukin-6 (IL-6) and interferon- $\gamma$ (IFN- $-\gamma$ ) have been shown to increase complement factor expression in many cell types, including the liver (17). The stimulatory effect on adipocyte C3 production has not been completely clarified, but the mRNA expression of C3 was shown to be moderately increased by IL-1 $\beta$ and perhaps also TNF- $\alpha$ in cultured mouse adipocytes (18), and our own observations showed that IL- 6 and IL-1 $\beta$ stimulated C3 mRNA expression in human (pre)adipocytes (Van Greevenbroek, unpublished observations). Besides low-grade inflammation, insulin resistance is also strongly associated with serum C3 levels $(5-7,19)$. 
In view of these considerations, both low-grade inflammation and insulin resistance may constitute mechanisms by which body fat is related to serum C3. To our knowledge, this has not yet been investigated in human epidemiological studies. We have therefore examined these issues in a population with a relatively high risk of type 2 diabetes and CVD.

\section{Methods and procedures}

\section{Subjects and study design}

The current study reports on cross-sectional analyses of data from The Cohort on Diabetes and Atherosclerosis Maastricht (CODAM). This study consists of 574 participants who were selected from a large cohort in the general population as described in detail elsewhere $(16,19,20)$. Briefly, subjects were included if they were of Caucasian ethnicity, $>40$ years-old, and met at least one of the following criteria: positive family history of type 2 diabetes (first degree), history of gestational diabetes, $\mathrm{BMI} \geq 25 \mathrm{~kg} / \mathrm{m}^{2}$, use of antihypertensive drugs, postprandial glucose $\geq 6.0 \mathrm{mmol} / \mathrm{l}$ or glycosuria. All subjects were extensively characterized with regard to their lifestyle, cardiovascular and metabolic profile during two visits to the University's metabolic research unit $(19,20)$.

Participants on insulin therapy $(n=13)$ or with missing data on anthropometrics, C3, or important covariates were excluded $(n=29)$ from analyses. Hence, the present study reports on 532 subjects. The study protocol was approved by the Medical Ethical Committee of the Maastricht University Medical Centre, and all subjects gave written informed consent.

\section{Measurements of body fat and fat distribution}

We measured height (in $\mathrm{cm}$ ) with subjects standing upright against a stadiometer, and body weight (to the nearest $100 \mathrm{~g}$ ) with subjects wearing only light indoor clothes and standing on calibrated electronic scales, to calculate subject's BMI (in $\mathrm{kg} / \mathrm{m}^{2}$ ). We used a flexible tape to measure WC at the level midway between the lateral lower rib margin and the anterior superior iliac spine, and $\mathrm{HC}$ at the level of the greater trochanter (both in $\mathrm{cm}$ ), and calculated WHR by dividing WC by HC. Sagittal diameter (in $\mathrm{cm}$ ) was measured at the spina iliaca anterior superior with subjects in supine position using a slide-gauge. In a subgroup of 393 subjects only, thickness of the bicipital, tricipital, subscapular and supra-iliac skinfolds was measured (in $\mathrm{mm}$ ) with a skinfold calliper (Servier, Neuilly-sur-Seine, France) on the non-dominant side. The average of 3 measurements was used in the analyses. We also calculated the sum of these 4 skinfolds ( $\sum 4 \mathrm{SKF}$ ) as an additional measure of overall fatness, and a skinfold ratio [i.e. (subscapular+suprailiac)/ $\Sigma 4 \mathrm{SKF}$ ] as a proxy measure of central (subcutaneous) fatness in these subjects. 


\section{C3, inflammatory markers and insulin resistance}

Participants were asked to stop their lipid-lowering drugs 14 days before and all other medication on the day before the visit to the research unit when venous blood samples were drawn after an overnight fast. Complement C3 levels were determined in serum by an automatic analyzer (Hitachi 912) with a Roche kit (Roche Diagnostics Netherlands BV) - inter-assay coefficient of variation of 2.1\% (19).

High sensitivity C-reactive protein (hs-CRP), haptogobin and ceruloplasmin were also measured in serum by an automatic analyzer (Hitachi 912) with a latex particleenhanced immunoturbidimetric assay (hs-CRP) or Tina-quant assay (all Roche Diagnostics) (19). Interleukin-6 (IL-6) (R\&D systems), serum amyloid A (SAA) (Biosource) and soluble inter-cellular adhesion molecule-1 (sICAM-1) (Biosource) were determined in EDTA plasma by ELISA (20).

Fasting plasma glucose was measured in NaF/KOx plasma with a hexokinase glucose- 6 phosphate dehydrogenase method (ABX Diagnostics). Fasting insulin concentrations were determined in EDTA plasma using a two-sided immunoradiometric test using paired monoclonal antibodies (Medgenix Diagnostics). Insulin resistance (IR) was estimated using the homeostasis model assessment (HOMA2-IR) calculator (www.dtu.ox.ac.uk).

\section{Other covariates}

Smoking habits were determined by an extensive questionnaire, as described previously in a study on smoking and C3 levels (21), and dichotomized (current smoker yes/no) for the present analyses. Alcohol consumption $(\mathrm{g} / \mathrm{d})$ was estimated from a validated food frequency questionnaire. Subjects underwent an oral glucose tolerance test (except those with established type 2 diabetes), and glucose metabolism status was defined according to the WHO 1999 criteria as previously described $(16,19,20)$. Prior CVD was defined as positive if participants: reported a history of myocardial infarction, coronary bypass surgery, percutaneous coronary interventions, stroke or non-traumatic limb amputation; had signs of myocardial infarction (Minnesota codes 1-1 or 1-2) or ischaemia (Minnesota codes 1-3, 4-1, 4-2, 4-3, 5-1, 5-2, 5-3 or 7-1) on a 12-lead ECG; and/or had an ankle-arm index was <0.9 in either leg (20). Alanine aminotransferase (ALT) was measured in EDTA-plasma with an Ecoline S+ assay (DiaSys Diagnostic Systems, Holzheim, Germany). Triglycerides were measured in serum as described before (20). Plasma creatinine levels were determined with a Jaffé diagnostic test (Roche Diagnostics), and glomerular filtration rate was estimated (eGFR) using the short Modification of Diet in Renal Disease equation (22).

\section{Statistical analysis}

Variables with a skewed distribution (i.e. HOMA2-IR, triglycerides, hs-CRP, IL-6, SAA, sICAM-1, ALT,) were In transformed prior to further analyses. General characteristics of 
the study population were compared across tertiles of serum C3 levels using analysis of variance (ANOVA) for continuous variables and $\chi 2$-test for discrete variables, and Pearson correlation coefficients between adiposity measures and other variables were calculated. A general inflammation score was calculated by averaging the Z-scores [i.e. (individuals' observed values - population mean)/SD] of six inflammatory markers (hs-CRP, haptoglobin, ceruloplasmin, IL-6, SAA, sICAM-1) (19). This composite score was used to provide a robust measure of low-grade inflammation, as it can be interpreted as six repeated measurements of the same construct (namely inflammation), thereby reducing measurement error. The use of such a score also reduces the influence of biological variability of each marker if assessed separately, and prevents multiple testing problems. Adiposity measurements and serum C3 were also standardized to enable comparison of the strength of the associations between several anthropometric measurements with $\mathrm{C} 3$. These associations were examined with the use of multiple linear regression models, and all associations were adjusted for age, sex, glucose metabolism status, prior CVD, eGFR, smoking status, alcohol consumption, and antihypertensive, lipid-lowering, and glucose-lowering medication (potential confounders or covariates) by including these variables in all regression models (forced entry).

The mediating role of low-grade inflammation and/or HOMA2-IR in the fully adjusted associations between estimates of body fat $(\mathrm{X})$ and $\mathrm{C} 3(\mathrm{Y})$ was examined with the use of simple and multiple mediation models, as illustrated in Figure 4.2.1 (23). Briefly, we investigated the extent to which the magnitude of the fully adjusted associations (depicted by the standardized regression coefficients - $\beta$ ) between adiposity measurements and C3 (total 'effect', represented by path c) could be apportioned into indirect or mediated 'effects' by the inflammation score and/or HOMA2-IR (represented by paths ab), and the remaining direct 'effect' of adiposity measurements on C3 (represented by path $\mathrm{c}^{\prime}$ ). In simple mediation analysis, only one mediator is investigated, and the product of the $\beta$ s representing paths $a$ and $b(a b)$ in Figure 4.2.1 indicates the mediated effect by that single mediator (either low-grade inflammation or HOMA2-IR). In multiple mediation analysis, both mediators are investigated simultaneously, and their individual mediated 'effects' are estimated independently of one another. Therefore, the independent or specific mediated 'effect' by low-grade inflammation is given by the product $a_{1} b_{1}$, and the independent mediated 'effect' by HOMA2-IR by $a_{2} b_{2}$. The magnitude of these mediated 'effects' is presented by $\beta$ s with respective (bias-corrected) $95 \% \mathrm{Cl}$ estimated by bootstrapping (5000 samples) because of its skewed distribution (23). This approach generates 5000 different datasets of $n=532$ subjects by randomly selecting subjects from the present dataset using sampling with replacement. The mediated 'effects' (ab) are assessed in all these 5000 datasets, and $95 \% \mathrm{Cl}$ are derived from the empirical distribution of these 5000 estimated 'effects'. We also quantified the magnitude of mediation relative to the 
total 'effects' in \% by dividing the mediated 'effect' by the overall association between adiposity measurement and serum $\mathrm{C} 3$ (i.e. $\beta_{\mathrm{ab}} / \beta_{\mathrm{c}} \times 100$ ).

We also investigated whether all associations reported herein differed between sexes by adding interaction terms between the adiposity measure and sex to the (fully adjusted) models. We found no such effect modification and therefore all results are presented for men and women combined. A two-sided $p$-value of $<0.05$ was considered statistically significant. All analyses were performed using the Statistical Package for Social Sciences for Windows, version 17.0 (SPSS Inc., Chicago, IL, USA).

\section{Results}

Characteristics of the study population across tertiles of serum C3 are shown in Table 4.2.1. Subjects in higher C3 tertiles generally had increasing levels of body fat, inflammatory markers, and HOMA2-IR and a worse overall metabolic and cardiovascular risk profile. Pearson correlations of adiposity measures with each other, and with metabolic and inflammatory variables are shown in Table 4.2.2.

All adiposity measurements, except the skinfold ratio, were significantly associated with serum C3 in linear regression models including age and sex (Table 4.2.3, paths $c_{\text {model } 1}$ ). After further adjustment for glucose metabolism status, prior CVD, eGFR, smoking status, alcohol use and medication, these associations were attenuated, but remained statistically significant (paths $c_{\text {model }} 2$ ). The sagittal diameter $(\beta=0.412$; $95 \% \mathrm{Cl} 0.333-0.490)$ and the WC $(\beta=0.383 ; 95 \% \mathrm{Cl} 0.302-0.464)$ showed the strongest associations with C3.

Mediation by low-grade inflammation and/or HOMA2-IR in the associations between all adiposity measures and $\mathrm{C} 3$ is shown in Table 4.2.3. For instance, the strength of the association between WC and $C 3 \quad(\beta=0.383 ; 95 \% \mathrm{Cl} 0.302-0.464)$, represented by path $c$ in Figure 4.2.1, was attenuated to $\beta=0.283(95 \% \mathrm{Cl} 0.205-0.360)$ when further adjusted for low-grade inflammation, or to $\beta=0.175(95 \% \mathrm{Cl} 0.085-0.265)$ when further adjusted for HOMA2-IR (Table 4.2.3; simple mediation analyses paths $\left.c^{\prime}\right)$. The magnitude of the mediation in absolute values was $\beta=0.101(95 \% \mathrm{Cl}$ $0.067-0.141)$ for low-grade inflammation and $\beta=0.208(95 \% \mathrm{Cl} 0.151-0.268)$ for HOMA2IR, explaining $26 \%$ and $54 \%$ of the association between WC and C3, respectively (paths $\mathrm{ab}$ ). When both mediators were simultaneously added to the regression model, the association between WC and C3 was attenuated to $0.115(95 \% \mathrm{Cl} 0.030-0.200)$ (Table 4.2.3; multiple mediation analyses - path $c^{\prime}$ ). The mediation by low-grade inflammation $(\beta=0.090 ; \quad 95 \% \mathrm{Cl} \quad 0.060-0.126)$ and by HOMA2-IR ( $\beta=0.179$; $95 \% \mathrm{Cl} 0.128-0.236)$ was independent of one another, with low-grade inflammation and HOMA2-IR each explaining, respectively, $23 \%$ and $47 \%$ of the association between WC and $\mathrm{C} 3$ (paths $\mathrm{a}_{1} \mathrm{~b}_{1}$ and $\mathrm{a}_{2} \mathrm{~b}_{2}$ ). 
A
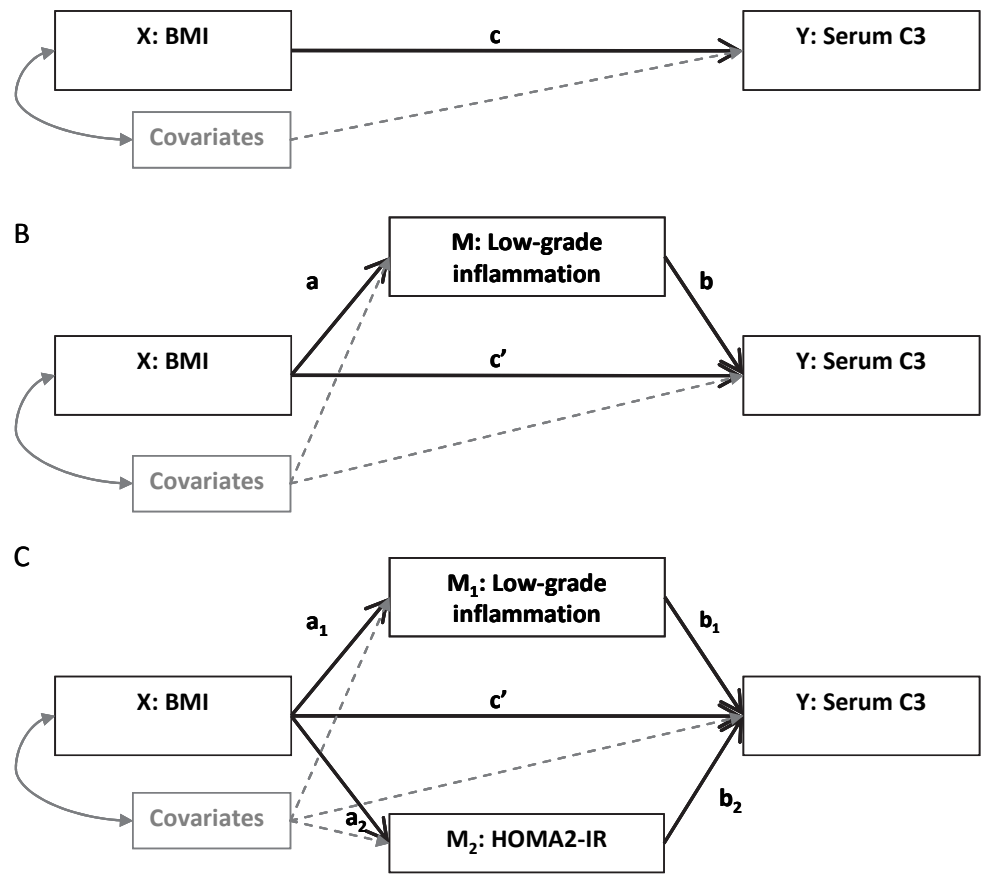

Figure 4.2.1. Example of simple and multiple mediation pathways as analysed in the present study

This figure illustrates how mediated effects were estimated in the association between body mass index (BMI), as an example of an adiposity measure, and C3. Panel A shows the association between BMI and serum C3 (dependent variable), adjusted for age, sex, glucose metabolism status, prior cardiovascular disease, estimated glomerular filtration rate, smoking status, alcohol consumption and antihypertensive, lipid-lowering, and glucose-lowering medication (covariates). Path $\mathrm{c}$ is equal to the standardized linear regression coefficient $(\beta)$, and represents the overall association of $\mathrm{BMI}$ with serum $\mathrm{C} 3$.

Panel B illustrates how the role of one single mediator is assessed in simple mediation analysis. Mediation is said to occur when (part of) the overall association between BMI and serum C3 is explained by (i.e. attenuated after adjustment for) a hypothesized intervening variable: mediator $\mathrm{M}$ (in this case: low-grade inflammation). In two separate regression models including all covariates, the association between BMI and low-grade inflammation (path a) and the association between low-grade inflammation and serum C3 independent of BMI (path b) are assessed. The product of regression coefficients $a$ and $b(a b)$ quantifies the indirect or mediated 'effect' by low-grade inflammation in the relation between BMI and serum $\mathrm{C} 3$. The part of the association not explained by mediator $\mathrm{M}$ is the direct 'effect' of BMI on serum $\mathrm{C} 3$ and is represented by path $C^{\prime}$. Analogously, the mediated effect of HOMA2-IR can be assessed in simple mediation analysis.

Panel $\mathrm{C}$ summarizes the coefficients of multiple mediation analysis. In addition to the analyses in panel B, there are now two mediators through which BMI may be associated with serum C3: low-grade inflammation (M1) and HOMA2-IR (M2). Associations between BMI and the mediators (paths $\mathrm{a}_{1}$ and $\mathrm{a}_{2}$ ) are similar to simple mediation analyses, but now the associations between mediators and serum C3 independent of one another and of BMI (paths $b_{1}$ and $b_{2}$ ) are assessed simultaneously in one regression model. Therefore, the independent mediated 'effect' by low-grade inflammation $\left(\mathrm{M}_{1}\right)$ in the relation between $\mathrm{BMI}$ and serum $\mathrm{C} 3$ is given by product $a_{1} b_{1}$, and the independent mediated 'effect' by HOMA2-IR $\left(M_{2}\right)$ is $a_{2} b_{2}$. The part of the association not explained by both mediating paths is the direct 'effect' ( $c$ ') of BMI on serum C3. 
Overall, the independent mediation by low-grade inflammation (19-27\%) and HOMA2-IR (37-56\%) was comparable across the associations between each adiposity measure (with the exception of the skinfold ratio) and C3 (Figure 4.2.2). Together these two mediators explained $60-83 \%$ of adiposity-related differences in serum C3.

Table 4.2.1 General characteristics of the overall study population ( $n=532)$, and across C3 tertiles.

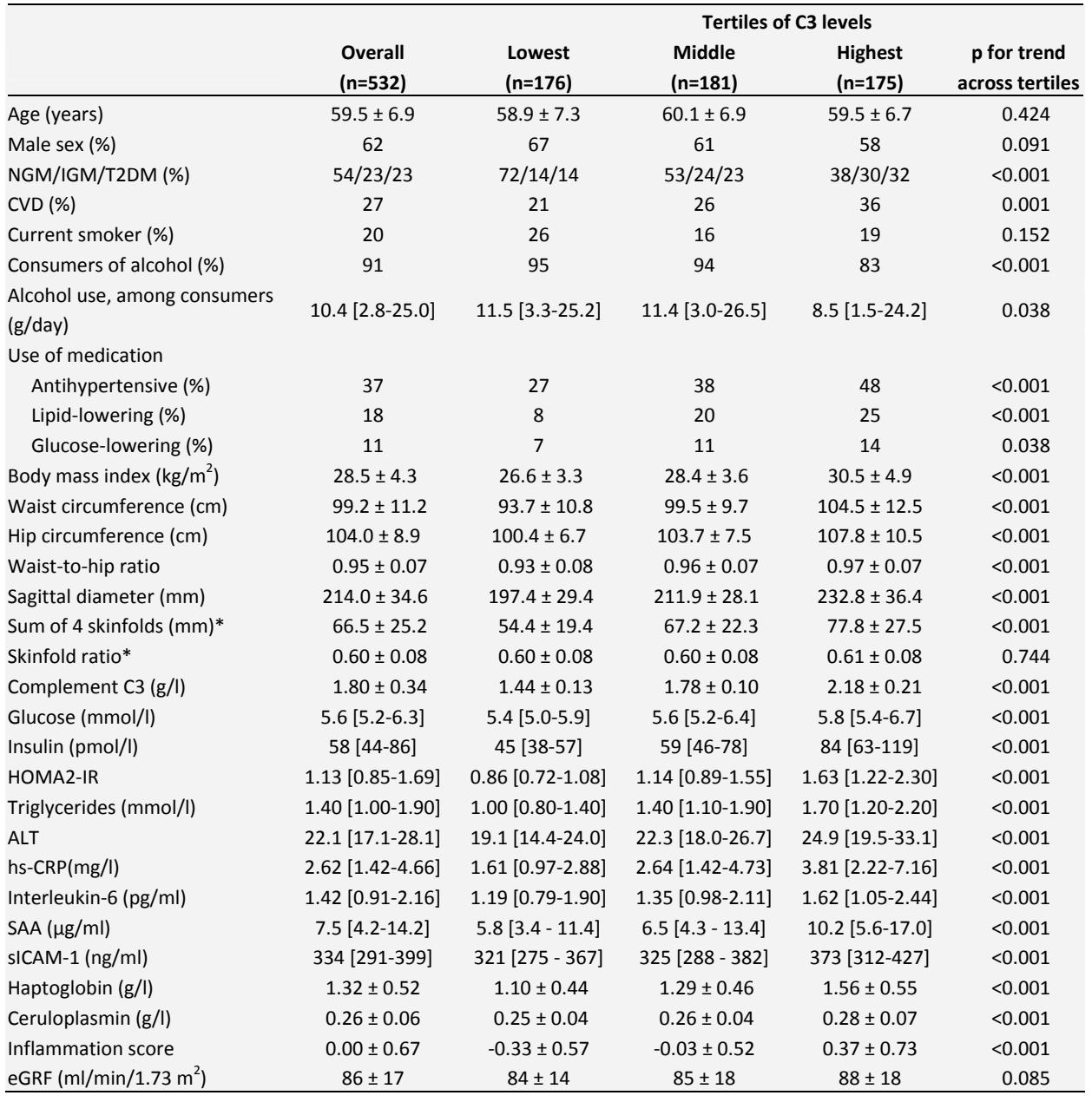

Data are expressed as mean $\pm \mathrm{SD}$, median [interquartile range] or percentages; the minimum and maximum of C3 tertiles were [1.01-1.62], [1.63-1.95] and [1.96-3.35] g/l respectively; NGM: normal glucose metabolism; IGM, impaired glucose metabolism; T2DM: type 2 diabetes mellitus; CVD: cardiovascular disease; HOMA2-IR: homeostasis model assessment insulin resistance; ALT: alanine aminotransferase; hs-CRP: high sensitivity C-reactive protein; SAA: serum amlyoid A; sICAM-1: soluble inter-cellular adhesion molecule-1; eGFR: estimated glomerular filtration rate; *Sum of 4 skinfolds and skinfold ratio were determined in a subgroup of 393 subjects only. 
Table 4.2.2 Pearson correlations of adiposity measures with metabolic and inflammatory variables.

\begin{tabular}{|c|c|c|c|c|c|c|c|}
\hline & BMI & WC & $\mathrm{HC}$ & WHR & SD & $\Sigma 4$ SKF & SKF ratio \\
\hline BMI & - & - & - & - & - & - & - \\
\hline Waist & $0.845+$ & - & - & - & - & - & - \\
\hline Hip & $0.849+$ & $0.741+$ & - & - & - & - & - \\
\hline WHR & $0.368+$ & $0.709+$ & 0.055 & - & - & - & - \\
\hline Sag Diam & $0.780+$ & $0.851 \dagger$ & $0.671+$ & $0.555^{\dagger}$ & - & - & - \\
\hline$\Sigma 4$ SKF & $0.681+$ & $0.491+$ & $0.648+$ & 0.065 & $0.480+$ & - & - \\
\hline SKF ratio & 0.015 & $0.241+$ & $-0.118^{*}$ & $0.471 \dagger$ & $0.215^{+}$ & $-0.246 \dagger$ & - \\
\hline C3 & $0.414+$ & $0.405^{\dagger}$ & $0.369+$ & $0.218^{\dagger}$ & $0.463+$ & $0.400+$ & 0.018 \\
\hline Glucose (In) & $0.300+$ & $0.363^{\dagger}$ & $0.216+$ & $0.315^{\dagger}$ & $0.358^{+}$ & $0.273+$ & 0.068 \\
\hline Insulin (In) & $0.583+$ & $0.601+$ & $0.474+$ & $0.392+$ & $0.565^{\dagger}$ & $0.491+$ & 0.032 \\
\hline HOMA2-IR (In) & $0.588+$ & $0.610^{+}$ & $0.475^{\dagger}$ & $0.405^{\dagger}$ & $0.576+$ & $0.496+$ & 0.041 \\
\hline ALT (In) & $0.304 t$ & $0.412+$ & $0.200^{\dagger}$ & $0.399+$ & $0.365^{+}$ & $0.163+$ & $0.261+$ \\
\hline Inflammation score & $0.283+$ & $0.224 \dagger$ & $0.280+$ & 0.039 & $0.308+$ & $0.331+$ & $-0.136+$ \\
\hline eGFR & $0.105^{*}$ & $0.145+$ & 0.065 & $0.146+$ & $0.150+$ & 0.072 & 0.046 \\
\hline
\end{tabular}

BMI: body mass index; Waist: waist circumference; Hip: hip circumference; WHR: waist-to-hip ratio; Sag Diam: sagittal diameter; C3: complement factor 3; HOMA2-IR: homeostasis model assessment insulin resistance; ALT: alanine aminotransferase; eGFR: estimated glomerular filtration rate. ${ }^{*} p<0.05 ;{ }^{\dagger}<<0.01$;

Table 4.2.3 Associations between adiposity measures and serum C3 (dependent variable), and the mediating role of low-grade inflammation and insulin resistance therein.

\begin{tabular}{|c|c|c|c|c|c|c|c|c|c|c|}
\hline \multirow[b]{3}{*}{ path§ } & \multicolumn{6}{|c|}{ Simple Mediation } & \multicolumn{4}{|c|}{ Multiple Mediation } \\
\hline & \multicolumn{3}{|c|}{ Inflammation score } & \multicolumn{3}{|c|}{ HOMA2-IR } & & & & \\
\hline & $\beta$ & $95 \% \mathrm{Cl}$ & $\%$ & $\beta$ & $95 \% \mathrm{Cl}$ & $\%$ & path§ & $\beta$ & $95 \% \mathrm{Cl}$ & $\%$ \\
\hline \multicolumn{11}{|c|}{ Body mass index } \\
\hline$c_{\text {(model1) }}$ & 0.412 & $0.334 ; 0.489 \ddagger$ & & 0.412 & $0.334 ; 0.489 \ddagger$ & & $C_{\text {(model1) }}$ & 0.412 & $0.334 ; 0.489 \ddagger$ & \\
\hline$c_{\text {(model2) }}$ & 0.320 & $0.243 ; 0.398 \ddagger$ & & 0.320 & $0.243 ; 0.398 \ddagger$ & & $c_{\text {(model2) }}$ & 0.320 & $0.243 ; 0.398 \ddagger$ & \\
\hline$c^{\prime}$ & 0.225 & $0.151 ; 0.298 \ddagger$ & & 0.115 & $0.031 ; 0.199+$ & & $c^{\prime}$ & 0.063 & $-0.020 ; 0.140$ & \\
\hline \multirow[t]{2}{*}{$a b$} & 0.096 & $0.062 ; 0.134 \ddagger$ & 30 & 0.205 & $0.155 ; 0.264 \ddagger$ & 64 & $a_{1} b_{1}$ (inflammation) & 0.084 & $0.054 ; 0.119 \ddagger$ & 26 \\
\hline & & & & & & & $\mathrm{a}_{2} \mathrm{~b}_{2}$ (HOMA2-IR) & 0.176 & $0.129 ; 0.231 \ddagger$ & 55 \\
\hline \multicolumn{11}{|c|}{ Waist circumference } \\
\hline $\mathrm{C}_{\text {(model1) }}$ & 0.475 & $0.396 ; 0.555 \ddagger$ & & 0.475 & $0.396 ; 0.555 \ddagger$ & & $\mathrm{C}_{\text {(model1) }}$ & 0.475 & $0.396 ; 0.555 \ddagger$ & \\
\hline $\mathrm{c}_{\text {(model2) }}$ & 0.383 & $0.302 ; 0.464 \ddagger$ & & 0.383 & $0.302 ; 0.464 \ddagger$ & & $\mathrm{c}_{\text {(model2) }}$ & 0.383 & $0.302 ; 0.464 \ddagger$ & \\
\hline$c^{\prime}$ & 0.283 & $0.205 ; 0.360 \ddagger$ & & 0.175 & $0.085 ; 0.265 \ddagger$ & & $c^{\prime}$ & 0.115 & $0.030 ; 0.200 \dagger$ & \\
\hline \multirow[t]{2}{*}{$a b$} & 0.101 & $0.067 ; 0.141 \ddagger$ & 26 & 0.208 & $0.151 ; 0.268 \ddagger$ & 54 & $a_{1} b_{1}$ (inflammation) & 0.090 & $0.060 ; 0.126 \ddagger$ & 23 \\
\hline & & & & & & & $\mathrm{a}_{2} \mathrm{~b}_{2}$ (HOMA2-IR) & 0.179 & $0.128 ; 0.236 \ddagger$ & 47 \\
\hline \multicolumn{11}{|c|}{ Hip circumference } \\
\hline $\mathrm{C}_{\text {(model1) }}$ & 0.361 & $0.280 ; 0.441 \ddagger$ & & 0.361 & $0.280 ; 0.441 \ddagger$ & & $\mathrm{C}_{\text {(model1) }}$ & 0.361 & $0.280 ; 0.441 \ddagger$ & \\
\hline $\mathrm{C}_{\text {(model2) }}$ & 0.281 & $0.203 ; 0.360 \ddagger$ & & 0.281 & $0.203 ; 0.360 \ddagger$ & & $\mathrm{C}_{\text {(model2) }}$ & 0.281 & $0.203 ; 0.360 \ddagger$ & \\
\hline$c^{\prime}$ & 0.191 & $0.117 ; 0.265 \ddagger$ & & 0.096 & $0.015 ; 0.177^{*}$ & & $c^{\prime}$ & 0.047 & $-0.029 ; 0.123$ & \\
\hline \multirow[t]{2}{*}{$a b$} & 0.090 & $0.058 ; 0.130 \ddagger$ & 32 & 0.186 & $0.138 ; 0.234 \ddagger$ & 66 & $a_{1} b_{1}$ (inflammation) & 0.077 & $0.047 ; 0.111 \ddagger$ & 27 \\
\hline & & & & & & & $a_{2} b_{2}$ (HOMA2-IR) & 0.157 & $0.115 ; 0.207 \ddagger$ & 56 \\
\hline \multicolumn{11}{|c|}{ Waist-to-hip ratio } \\
\hline $\mathrm{C}_{\text {(model1) }}$ & 0.460 & $0.358 ; 0.562 \ddagger$ & & 0.460 & $0.358 ; 0.562 \ddagger$ & & $\mathrm{C}_{\text {(model1) }}$ & 0.460 & $0.358 ; 0.562 \ddagger$ & \\
\hline $\mathrm{c}_{\text {(model2) }}$ & 0.347 & $0.247 ; 0.448 \ddagger$ & & 0.347 & $0.247 ; 0.448 \ddagger$ & & $\mathrm{c}_{\text {(model2) }}$ & 0.347 & $0.247 ; 0.448 \ddagger$ & \\
\hline$c^{\prime}$ & 0.268 & $0.176 ; 0.360 \ddagger$ & & 0.164 & $0.066 ; 0.261 \dagger$ & & $c^{\prime}$ & 0.133 & $0.046 ; 0.224 \dagger$ & \\
\hline \multirow[t]{2}{*}{$a b$} & 0.079 & $0.036 ; 0.130 \ddagger$ & 23 & 0.184 & $0.129 ; 0.245 \ddagger$ & 53 & $a_{1} b_{1}$ (inflammation) & 0.065 & $0.029 ; 0.106 \ddagger$ & 19 \\
\hline & & & & & & & $\mathrm{a}_{2} \mathrm{~b}_{2}$ (HOMA2-IR) & 0.150 & $0.102 ; 0.206 \ddagger$ & 43 \\
\hline
\end{tabular}


Table 4.2.3 (continued)

\begin{tabular}{|c|c|c|c|c|c|c|c|c|c|c|}
\hline \multirow[b]{3}{*}{ path§ } & \multicolumn{6}{|c|}{ Simple Mediation } & \multicolumn{4}{|c|}{ Multiple Mediation } \\
\hline & \multicolumn{3}{|c|}{ Inflammation score } & \multicolumn{3}{|c|}{ HOMA2-IR } & & & & \\
\hline & $\beta$ & $95 \% \mathrm{Cl}$ & $\%$ & $\beta$ & $95 \% \mathrm{Cl}$ & $\%$ & path§ & $\beta$ & $95 \% \mathrm{Cl}$ & $\%$ \\
\hline \multicolumn{11}{|c|}{ Sagittal diameter } \\
\hline $\mathrm{C}_{(\text {model1) }}$ & 0.503 & $0.428 ; 0.579 \ddagger$ & & 0.503 & $0.428 ; 0.579 \ddagger$ & & $\mathrm{C}_{(\text {model1) }}$ & 0.503 & $0.428 ; 0.579 \ddagger$ & \\
\hline $\mathrm{C}_{(\text {model2) }}$ & 0.412 & $0.333 ; 0.490 \ddagger$ & & 0.412 & $0.333 ; 0.490 \ddagger$ & & $\mathrm{C}_{(\text {model2) }}$ & 0.412 & $0.333 ; 0.490 \ddagger$ & \\
\hline$c^{\prime}$ & 0.304 & $0.227 ; 0.380 \ddagger$ & & 0.238 & $0.153 ; 0.322 \ddagger$ & & $\mathrm{c}^{\prime}$ & 0.163 & $0.082 ; 0.244 \ddagger$ & \\
\hline \multirow[t]{2}{*}{$a b$} & 0.108 & $0.074 ; 0.149 \ddagger$ & 26 & 0.174 & $0.124 ; 0.230 \ddagger$ & 42 & $\mathrm{a}_{1} \mathrm{~b}_{1}$ (inflammation) & 0.096 & $0.064 ; 0.133 \ddagger$ & 23 \\
\hline & & & & & & & $\mathrm{a}_{2} \mathrm{~b}_{2}$ (HOMA2-IR) & 0.152 & $0.107 ; 0.206 \ddagger$ & 37 \\
\hline \multicolumn{11}{|c|}{ Sum of 4 skinfolds } \\
\hline $\mathrm{C}_{\text {(model1) }}$ & 0.461 & $0.360 ; 0.562 \ddagger$ & & 0.461 & $0.360 ; 0.562 \ddagger$ & & $\mathrm{C}_{\text {(model1) }}$ & 0.461 & $0.360 ; 0.562 \ddagger$ & \\
\hline $\mathrm{C}_{(\text {model2) }}$ & 0.362 & $0.255 ; 0.468 \ddagger$ & & 0.362 & $0.255 ; 0.468 \ddagger$ & & $\mathrm{C}_{\text {(model2) }}$ & 0.362 & $0.255 ; 0.468 \ddagger$ & \\
\hline$c^{\prime}$ & 0.264 & $0.167 ; 0.364 \ddagger$ & & 0.152 & $0.038 ; 0.265^{\dagger}$ & & $c^{\prime}$ & 0.091 & $-0.016 ; 0.197$ & \\
\hline \multirow[t]{2}{*}{$a b$} & 0.098 & $0.053 ; 0.150 \ddagger$ & 27 & 0.210 & $0.144 ; 0.283 \ddagger$ & 58 & $\mathrm{a}_{1} \mathrm{~b}_{1}$ (inflammation) & 0.088 & $0.048 ; 0.136 \ddagger$ & 24 \\
\hline & & & & & & & $\mathrm{a}_{2} \mathrm{~b}_{2}$ (HOMA2-IR) & 0.183 & $0.122 ; 0.249 \ddagger$ & 51 \\
\hline \multicolumn{11}{|c|}{ Skinfold ratio } \\
\hline $\mathrm{C}_{\text {(model1) }}$ & 0.080 & $-0.041 ; 0.201$ & & 0.080 & $-0.041 ; 0.201$ & & $\mathrm{C}_{\text {(model1) }}$ & 0.080 & $-0.041 ; 0.201$ & \\
\hline $\mathrm{C}_{(\text {model2) }}$ & 0.083 & $-0.029 ; 0.196$ & & 0.083 & $-0.029 ; 0.196$ & & $\mathrm{C}_{\text {(model2) }}$ & 0.083 & $-0.029 ; 0.196$ & \\
\hline$c^{\prime}$ & 0.085 & $-0.015 ; 0.185$ & & 0.071 & $-0.029 ; 0.171$ & & $c^{\prime}$ & 0.075 & $-0.017 ; 0.166$ & \\
\hline \multirow[t]{2}{*}{$a b$} & -0.002 & $-0.052 ; 0.045$ & - & 0.012 & $-0.038 ; 0.064$ & - & $\mathrm{a}_{1} \mathrm{~b}_{1}$ (inflammation) & -0.002 & $-0.040 ; 0.038$ & \\
\hline & & & & & & & $\mathrm{a}_{2} \mathrm{~b}_{2}$ (HOMA2-IR) & 0.010 & $-0.032 ; 0.055$ & - \\
\hline
\end{tabular}

Paths $\mathbf{c}$ show the magnitude of the overall associations between adiposity measures and C3 in standardized regression coefficients $(\beta)$, which reflect the change in C3 (in SD) per SD increase in the adiposity measurements, for the following models: Model 1: adjusted for age and sex; Model 2: model $1+$ adjusted for glucose metabolism status, prior cardiovascular disease, estimated glomerular filtration rate, smoking status, alcohol consumption, and antihypertensive, lipid-lowering, and glucose-lowering medication. In the fully adjusted associations (model 2), path $\mathbf{c}^{\prime}$ represents the direct association between adiposity measure and C3 that is not explained by the mediator(s) considered; paths ab represent the portion of $\mathbf{c}$, in absolute values and in percentages (i.e. $\beta_{\mathrm{ab}} / \beta_{\mathrm{c}} \times 100 \%$ ), that is explained by either low-grade inflammation or HOMA2-IR (simple mediation analyses); paths $\mathbf{a}_{\mathbf{1}} \mathbf{b}_{\mathbf{1}}$ and $\mathbf{a}_{\mathbf{2}} \mathbf{b}_{\mathbf{2}}$ represent the portion of $\mathbf{c}$, in absolute values and in percentages (i.e. $\beta_{\mathrm{a} 1 \mathrm{~b} 1 / \beta \mathrm{c}} \times 100 \%$ and i.e. $\beta_{\mathrm{a} 2 \mathrm{~b} 2} / \beta_{\mathrm{c}} \times 100 \%$ ), that is explained by low-grade inflammation and HOMA2-IR, respectively, independently of one another (multiple mediation analyses); ${ }^{*} p<0.05 ;+p<0.01$; $\neq p<0.001$; See also Figure 4.2.1.

\section{Additional analyses}

We also evaluated (simple) mediation by the six individual inflammatory markers and the inflammation score in the associations of waist circumference and sagittal diameter with C3 (Figure 4.2.3). Mediation by individual markers ranged from 3\% (for IL-6) to $29 \%$ (for hs-CRP), but all markers attenuated the association between adiposity measures and C3 to some extent. 


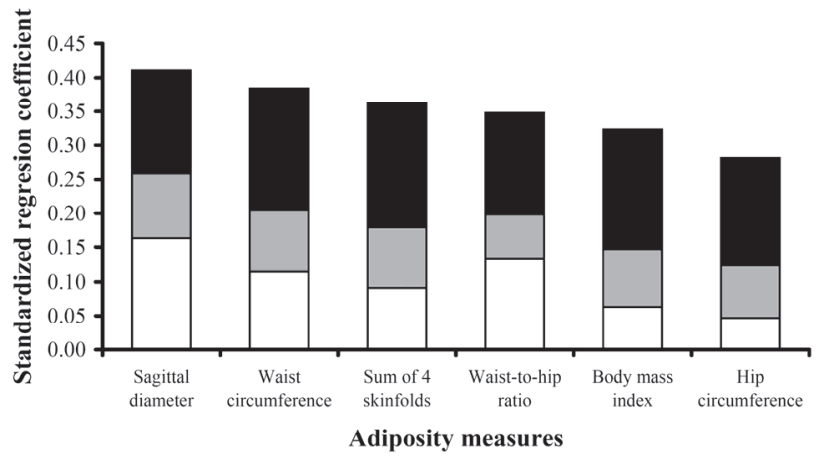

$\square$ Unexplained $\square$ Inflammation score $\mathbf{B O M A 2 - I R}$

Figure 4.2.2 Associations between adiposity measures and serum C3 (paths c), ordered from strongest to weakest as indicated by the size of the bars; portions of the overall associations independently explained by low-grade inflammation (paths $a_{1} b_{1}$ ) are given in grey (19-27\%) and by HOMA2-IR (paths $a_{2} b_{2}$ ) are given in black (37-56\%). Together, these mediators explained 60 to $83 \%$ of the adiposity-related increases in C3. Portions that remained unexplained are given in white (paths c').
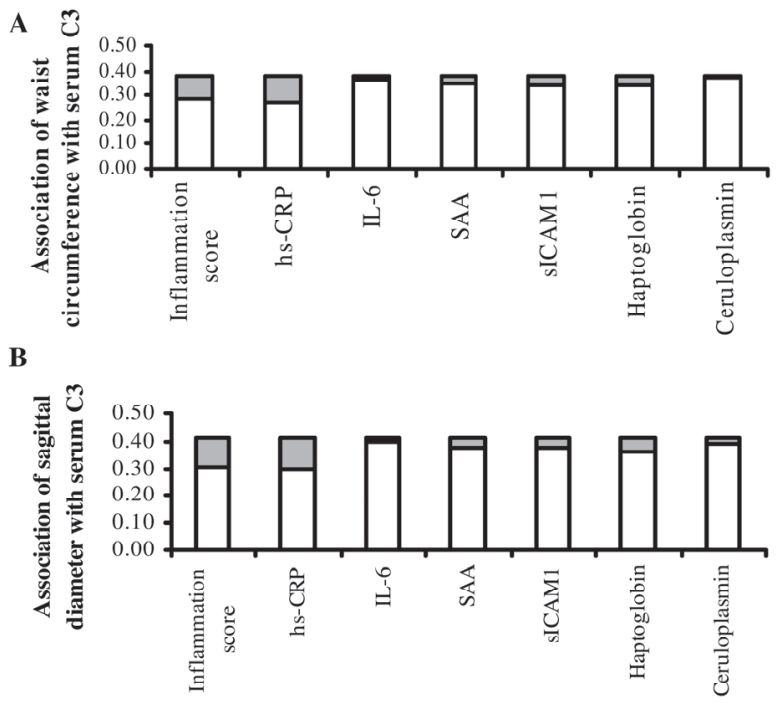

Markers of low-grade inflammation

$\square$ Unexplained $\mathbf{D}$ Explained by marker

Figure 4.2.3 Simple mediation by the inflammation score and the individual inflammatory markers in the (adjusted) associations of waist circumference (panel A) and sagittal diameter (panel B) with serum C3. hs-CRP: high sensitivity C-reactive protein; IL-6: interleukin-6; SAA: serum amlyoid A; sICAM-1: soluble inter-cellular adhesion molecule-1. 
In previous work, we have shown that plasma alanine aminotransferase (ALT) explained about $15 \%$ of the cross-sectional association between HOMA2-IR and serum C3 (19). Therefore, we also performed the mediation analyses in models that were additionally adjusted for plasma ALT. In these models, the mediation by low-grade inflammation was not appreciably altered (20-30\%), and the mediation by HOMA2-IR was slightly attenuated (28-48\%), but remained to a great extent independent of ALT. Furthermore, the presence of infectious or inflammatory diseases at the time of blood sampling may have influenced serum C3 concentrations. However, all observed associations and mediated 'effects' did not materially change in an additional analysis excluding subjects with serum hs-CRP $>10 \mathrm{mg} / \mathrm{L}$ ( $\mathrm{n}=41$; data not shown). In addition, similar associations were observed when subjects with self-reported (history of) renal, intestinal, bile duct or hepatic diseases were excluded ( $n=49$; data not shown). C3 concentrations may also be determined by female sex hormones $(24,25)$, but additional adjustment for menopausal status (79\% postmenopausal) or hormone replacement therapy (9\%) in women did not influence our findings (data not shown). Also, additional adjustment for physical activity and daily energy intake ( $n=527$ subjects) did not change our results (data not shown).

Finally, in sensitivity analyses confined to the subgroup with skinfold data only, associations of BMI, waist and hip circumferences, and sagittal diameter with C3 were similar to those reported in the overall population (data not shown).

\section{Discussion}

The present study has two main findings. First, we confirmed the independent association of several estimates of body fat with serum C3 levels. As novel findings, we showed that a large part of this association ( $60-80 \%)$ was explained, i.e. mediated, by fat-related low-grade inflammation ( $20-25 \%)$ and insulin resistance ( $\sim 40-55 \%)$, independently of one another.

The association of body fat with serum $\mathrm{C} 3$ levels has been demonstrated for several individual measurements of body fat, such as BMI, WC, HC, WHR, and the sum of body skinfolds - but not for the skinfold ratio - in several populations, including healthy adolescents $(5,10,12)$. The present study found similar associations, with the strongest associations being observed with measures of central fat (i.e. sagittal diameter and WC), but not with the ratio of truncal to total skinfolds. These findings suggest that the amount of abdominal fat may be more strongly associated with differences in serum C3 than a central subcutaneous fat distribution. Although the subgroup with available data on skinfolds $(n=393)$ had slightly lower C3 levels than the overall study population $(\mathrm{n}=532$ ) (mean difference of $0.056 \mathrm{~g} / \mathrm{l}$ ), they did not differ in other adiposity measures and covariates, and showed similar associations between these adiposity measures and C3. Hence, we believe this subgroup to be a representative selection of the overall 
study population, and our findings regarding the lack of association between the skinfolds ratio and C3 not to have been affected by selection bias.

This study not only confirmed the strong relationship of body fat with serum C3, but also explored potential mechanisms to explain this association. There is growing evidence that obesity is accompanied by local and systemic low-grade inflammation (14-16), and several pro-inflammatory cytokines like TNF- $\alpha$, IL-1, IL-6 and IFN- $\gamma$ have been shown to increase the production of $\mathrm{C} 3$ in many cell types in vitro $(17,18)$. The moderate, but significant mediation (19-27\%) found in the present epidemiological study supports the concept that pro-inflammatory cytokines may increase obesityassociated C3 production. In the current design, we could, however, not differentiate between C3 derived from liver and from adipose tissue, since higher serum C3 concentrations that are observed in association with low grade inflammation may originate directly from adipose tissue, from the (steatotic) liver, or from both.

In addition, HOMA2-IR was an even stronger mediator, explaining 37-56\% of the relation between body fat and serum C3. This is in line with the strong association found between insulin resistance and serum C3 in several other studies $(5-7,19)$. Notably, the mediation by HOMA2-IR was to a large extent independent of, and did not overlap with that by low-grade inflammation. Some investigators have postulated that the association between HOMA2-IR and serum C3 could be attributed to a common predecessor, such as obesity-related low-grade inflammation $(5,6)$. If this had been the case, the mediated 'effects' $\left(\beta_{\mathrm{ab}}\right)$ of low-grade inflammation and HOMA2-IR in simple mediation analyses should have been considerably attenuated in multiple mediation analyses $\left(\beta_{\mathrm{a} 1 \mathrm{~b} 1}\right.$ and $\left.\beta_{\mathrm{a} 2 \mathrm{~b} 2}\right)$, in support of a shared 'effect'. We have previously shown that the association between insulin resistance and C3 was, to a large extent, independent of low-grade inflammation (19), and now that both pathways independently link adiposity to $\mathrm{C} 3$.

A possible explanation for the inflammation-independent mediation by insulin resistance is that insulin is able to directly attenuate C3 transcription and production, as has been shown in $\mathrm{H}-35$ liver cells in vitro, but not yet in adipocytes (26). Such an inhibitory effect of insulin on C3 production may be lost in an insulin-resistant state. Insulin action in euglycaemic hyperinsulinaemic clamps was indeed inversely related to both visceral and subcutaneous adipose tissue C3 mRNA expression (11). However, several small clamp studies found no acute changes in adipose tissue C3 mRNA expression or serum C3 after a short period of hyperinsulinemia $(5,11)$. Insulin has been shown to exert both stimulatory and inhibiting effects at the C3 secretion level in adipocytes in vitro, so there may actually be a complex regulation of C3 secretion by insulin. Another potential mechanism that might explain the increased serum C3 by insulin resistance is the (postprandial) insulin resistance-associated hypertriglyceridemia. Chylomicrons have been shown to greatly augment the production of C3 by adipocytes in vitro (27). Moreover, acute increases in circulating C3 after an oral fat load were higher in insulin-resistant persons, and accompanied by higher 
postprandial circulating triglycerides and non-esterified fatty acids (7). Addition of glucose to the oral fat load reduced this C3 response in insulin-sensitive persons (28). The glucose-related insulin response might ensure an effective fatty acid trapping in adipocytes and efficient chylomicron clearance, thereby preventing chylomicroninduced C 3 production. On the other hand, these data are also compatible with a direct inhibition of C3 production by insulin, as discussed above. Finally, the mediation by insulin resistance was slightly attenuated (to 28-48\%) when adjustment for plasma ALT was taken into account. In agreement with the previous study (19), this might indicate that a small portion of the mediation by insulin resistance is in fact due to related differences in liver fat content or hepatocellular damage.

Although low-grade inflammation and insulin resistance together explained up to $80 \%$ of adiposity-related differences in $\mathrm{C} 3$, a small, but statistically significant portion of the association remained unexplained, suggesting other mechanisms to be involved. C3 is also the precursor protein for C3a-desarg, also known as acylation stimulating protein (ASP), which promotes incorporation of non-esterified fatty acids into triglycerides in adipocytes (9). An additional mechanism via which body fat relates to C3 that is not explained by low grade inflammation and/or insulin resistance, might be that the elevated C3 levels are indicative of ASP resistance in adipocytes, analogous to the hyperinsulinaemia in the presence of insulin resistance $(7,9)$. Not only TNF- $\alpha$, but also fatty acids and sex steroids have been shown to cause a downregulation of the ASP receptor, and interfere with downstream ASP signalling, in support of this hypothesis. However, obesity-associated changes in serum C3 may also occur via currently unknown mechanisms in cells other than adipocytes.

A major limitation of the present study is the cross-sectional study design, which does not allow us to draw definite conclusions on causality. However, we used multiple mediation techniques to confirm hypothesized causal pathways, based on the current (experimental) literature, in humans in vivo. This adds to a more comprehensive understanding of aetiology by providing additional insight into potential explanatory pathways underlying the adiposity-C3 associations. In theory, reverse causation cannot be fully excluded. Although C3 cleavage products such as C3a may act as a chemoattractants and proinflammatory agents, C3 itself has no known inflammatory or metabolic effects, and C3 levels are not necessarily correlated with C3a (9). Therefore, a reverse causation hypothesis in which serum C3 itself causes inflammation or insulin resistance is not likely.

In addition, a possible drawback of averaging inflammatory markers into a score is that it does not take into account potential differences in biological importance of individual markers, but instead focuses on the overall effect of the inflammatory state. This approach prevents that too much emphasis is laid on the individual inflammatory marker that, possibly by chance or by differences in measurement error, showed the strongest individual mediation effect, when in fact it is the underlying general inflammation process that is most relevant for the observed mediation. In the 
additional analyses, we indeed showed that the mediation by all individual markers is in the same direction as the overall score. In our current study we focussed on classical inflammation mediators. There are, however, various other inflammatory markers that may also contribute to the total inflammatory burden. For instance, the presence of dysfunctional high-density lipoprotein (HDL) may also contribute to low-grade inflammation, particularly in our metabolically stressed population, and hence increase C3 levels (29). We believe that a lack of anti-inflammatory effect that is associated with HDL dysfunction will at least partially be represented by higher inflammatory markers and a higher inflammation score in our study. However, HDL dysfunction might also act as an independent determinant of $\mathrm{C} 3$ levels that was unfortunately not measured in our study. Finally, our study was conducted in middle-aged and elderly, Caucasian subjects with an increased risk for metabolic and cardiovascular disease, and therefore, extrapolation of these data to the general population or other ethnicities should be done with caution.

In conclusion, we have shown that low-grade inflammation and insulin resistance may represent two independent pathways that together account for up to $80 \%$ of the association between body fat and increased serum C3 levels. Within this study design, we were unable to determine whether changes in serum C3 concentration originate from liver or fat. Further in vivo and in vitro research is needed to dissect the effects of cytokines and insulin on C3 transcription and production in hepatocytes and adipocytes, and to identify the source(s) that contribute(s) to changes in serum C3 in cardiometabolic diseases. 


\section{References}

1. Muscari A, Bozzoli C, Puddu GM, Sangiorgi Z, Dormi A, Rovinetti C, Descovich GC, Puddu P: Association of serum C3 levels with the risk of myocardial infarction. Am J Med 1995;98:357-364

2. Engstrom G, Hedblad B, Eriksson KF, Janzon L, Lindgarde F: Complement C3 is a risk factor for the development of diabetes: a population-based cohort study. Diabetes 2005;54:570-575

3. Engstrom G, Hedblad B, Berglund G, Janzon L, Lindgarde F: Plasma levels of complement C3 is associated with development of hypertension: a longitudinal cohort study. J Hum Hypertens 2007;21:276-282

4. Onat A, Hergenc G, Can G, Kaya Z, Yuksel H: Serum complement C3: a determinant of cardiometabolic risk, additive to the metabolic syndrome, in middle-aged population. Metabolism 2010;59:628-634

5. Weyer C, Tataranni PA, Pratley RE: Insulin action and insulinemia are closely related to the fasting complement C3, but not acylation stimulating protein concentration. Diabetes Care 2000;23:779-785

6. Muscari A, Antonelli S, Bianchi G, Cavrini G, Dapporto S, Ligabue A, Ludovico C, Magalotti D, Poggiopollini G, Zoli M: Serum C3 is a stronger inflammatory marker of insulin resistance than Creactive protein, leukocyte count, and erythrocyte sedimentation rate: comparison study in an elderly population. Diabetes Care 2007;30:2362-2368

7. van Oostrom AJ, Alipour A, Plokker TW, Sniderman AD, Cabezas MC: The metabolic syndrome in relation to complement component 3 and postprandial lipemia in patients from an outpatient lipid clinic and healthy volunteers. Atherosclerosis 2007;190:167-173

8. Naughton MA, Botto M, Carter MJ, Alexander GJ, Goldman JM, Walport MJ: Extrahepatic secreted complement C3 contributes to circulating C3 levels in humans. J Immunol 1996;156:3051-3056

9. Cianflone K, Xia Z, Chen LY: Critical review of acylation-stimulating protein physiology in humans and rodents. Biochim Biophys Acta 2003;1609:127-143

10. Gabrielsson BG, Johansson JM, Lonn M, Jernas M, Olbers T, Peltonen M, Larsson I, Lonn L, Sjostrom L, Carlsson B, Carlsson LM: High expression of complement components in omental adipose tissue in obese men. Obes Res 2003;11:699-708

11. Koistinen HA, Vidal H, Karonen SL, Dusserre E, Vallier P, Koivisto VA, Ebeling P: Plasma acylation stimulating protein concentration and subcutaneous adipose tissue C3 mRNA expression in nondiabetic and type 2 diabetic men. Arterioscler Thromb Vasc Biol 2001;21:1034-1039

12. Warnberg J, Nova E, Moreno LA, Romeo J, Mesana MI, Ruiz JR, Ortega FB, Sjostrom M, Bueno M, Marcos A: Inflammatory proteins are related to total and abdominal adiposity in a healthy adolescent population: the AVENA Study. Am J Clin Nutr 2006;84:505-512

13. Pomeroy C, Mitchell J, Eckert E, Raymond N, Crosby R, Dalmasso AP: Effect of body weight and caloric restriction on serum complement proteins, including Factor D/adipsin: studies in anorexia nervosa and obesity. Clin Exp Immunol 1997;108:507-515

14. Yudkin JS, Stehouwer CD, Emeis JJ, Coppack SW: C-reactive protein in healthy subjects: associations with obesity, insulin resistance, and endothelial dysfunction: a potential role for cytokines originating from adipose tissue? Arterioscler Thromb Vasc Biol 1999;19:972-978

15. Trayhurn P, Wood IS: Adipokines: inflammation and the pleiotropic role of white adipose tissue. Br J Nutr 2004;92:347-355

16. Thewissen MM, Damoiseaux JG, Duijvestijn AM, van Greevenbroek MM, van der Kallen CJ, Feskens EJ, Blaak EE, Schalkwijk CG, Stehouwer CD, Cohen Tervaert JW, Ferreira I: Abdominal fat mass is associated with adaptive immune activation: the CODAM Study. Obesity (Silver Spring) 2011;19:1690-1698

17. Volanakis JE: Transcriptional regulation of complement genes. Annu Rev Immunol 1995;13:277-305

18. Peake PW, O'Grady S, Pussell BA, Charlesworth JA: Detection and quantification of the control proteins of the alternative pathway of complement in 3T3-L1 adipocytes. Eur J Clin Invest 1997;27:922-927

19. van Greevenbroek MM, Jacobs M, van der Kallen CJ, Vermeulen VM, Jansen EH, Schalkwijk CG, Ferreira I, Feskens EJ, Stehouwer CD: The cross-sectional association between insulin resistance and circulating complement C3 is partly explained by plasma alanine aminotransferase, independent of central obesity and general inflammation (the CODAM study). Eur J Clin Invest 2011;41:372-379 
20. Jacobs M, van Greevenbroek MM, van der Kallen CJ, Ferreira I, Blaak EE, Feskens EJ, Jansen EH, Schalkwijk CG, Stehouwer CD: Low-grade inflammation can partly explain the association between the metabolic syndrome and either coronary artery disease or severity of peripheral arterial disease: the CODAM study. Eur J Clin Invest 2009;39:437-444

21. van Greevenbroek MM, Jacobs M, van der Kallen CJ, Blaak EE, Jansen EH, Schalkwijk CG, Feskens EJ, Stehouwer CD: Human plasma complement C3 is independently associated with coronary heart disease, but only in heavy smokers (the CODAM study). Int J Cardiol 2012;154:158-162

22. Levey AS, Bosch JP, Lewis JB, Greene T, Rogers N, Roth D: A more accurate method to estimate glomerular filtration rate from serum creatinine: a new prediction equation. Modification of Diet in Renal Disease Study Group. Ann Intern Med 1999;130:461-470

23. Preacher KJ, Hayes AF: Asymptotic and resampling strategies for assessing and comparing indirect effects in multiple mediator models. Behav Res Methods 2008;40:879-891

24. Li SH, Huang HL, Chen YH: Ovarian steroid-regulated synthesis and secretion of complement C3 and factor $B$ in mouse endometrium during the natural estrous cycle and pregnancy period. Biol Reprod 2002;66:322-332

25. Yilmazer M, Fenkci V, Fenkci S, Aktepe O, Sonmezer M, Kurtay G: Association of serum complement $(\mathrm{C} 3, \mathrm{C} 4)$ and immunoglobulin (IgG, IgM) levels with hormone replacement therapy in healthy postmenopausal women. Hum Reprod 2003;18:1531-1535

26. Campos SP, Baumann $\mathrm{H}$ : Insulin is a prominent modulator of the cytokine-stimulated expression of acute-phase plasma protein genes. Mol Cell Biol 1992;12:1789-1797

27. Scantlebury T, Maslowska M, Cianflone K: Chylomicron-specific enhancement of acylation stimulating protein and precursor protein C3 production in differentiated human adipocytes. J Biol Chem 1998;273:20903-20909

28. van Oostrom AJ, van Dijk H, Verseyden C, Sniderman AD, Cianflone K, Rabelink TJ, Castro Cabezas M: Addition of glucose to an oral fat load reduces postprandial free fatty acids and prevents the postprandial increase in complement component 3. Am J Clin Nutr 2004;79:510-515

29. Onat A, Can G, Rezvani R, Cianflone K: Complement C3 and cleavage products in cardiometabolic risk. Clin Chim Acta 2011;412:1171-1179 


\section{Chapter 4.3}

Complement factor 3 is associated with insulin resistance and with incident type 2 diabetes mellitus over a 7-year follow-up period

Nick Wlazlo, Marleen MJ van Greevenbroek, Isabel Ferreira, Edith JM Feskens, Carla JH van der Kallen, Casper G Schalkwijk, Bert Bravenboer, Coen DA Stehouwer Accepted Diabetes Care 


\section{Abstract}

\section{Objective}

The complement system increasingly recognized as a regulator of immune and inflammatory homeostasis, but longitudinal studies focussing on complement as a determinant of type 2 diabetes mellitus (T2DM) and insulin resistance (IR) are scarce. Therefore, we prospectively investigated the association of plasma C3 with (estimates of) IR in muscle, liver, and adipocytes, as well as with glucose tolerance, including incident T2DM.

\section{Research design and methods}

Fasting C3, non-esterified fatty acids, glucose and insulin (the latter two during oral glucose tolerance tests), were measured at baseline $(n=545)$ and after 7 years' follow-up $(n=394)$ in a prospective cohort study.

\section{Results}

Over the 7-year period, C3 levels (per $0.1 \mathrm{~g} / \mathrm{L}$ ) were longitudinally associated with higher HOMA2IR $(\beta=15.2 \%[95 \% \mathrm{Cl} 12.9-17.6])$, hepatic IR $(\beta=6.1 \%$ [95\% Cl 4.7-7.4]), adipocyte IR ( $\beta=16.0 \%$ [95\% Cl 13.0-19.1]), fasting glucose ( $\beta=1.8 \%$ [95\% Cl 1.2-2.4]), $2 \mathrm{~h}$ glucose $(\beta=5.2 \%$ [95\% $\mathrm{Cl} 3.7-6.7])$, and AUCglucose $(\beta=3.6 \%[95 \% \mathrm{Cl} 2.7-4.6])$. Also, greater changes in $\mathrm{C} 3$ were associated with greater changes in the IR measures, but not glucose tolerance. Moreover, baseline C3 was associated with the 7-year incidence of T2DM (OR 1.5 [95\% Cl 1.1-2.0]).

\section{Conclusions}

Changes in C3 were associated with changes in several measures of IR, and may reflect progression of metabolic dysregulation, that eventually leads to abnormalities in glucose tolerance and T2DM. 


\section{Introduction}

Plasma complement factor 3 (C3) is an emerging risk marker for cardiovascular and metabolic diseases (1-3), and systemic C3 concentrations are closely linked to several measures of body fat and components of the metabolic syndrome (4-6). Complement C3 is produced mainly by the liver (7), but other production sites, such as adipose tissue, may also contribute to systemic C3 levels (8-10). C3 is the central component of the complement system, and activation via any of the three major complement pathways results in cleavage of $\mathrm{C} 3$ into $\mathrm{C} 3 \mathrm{a}$ and $\mathrm{C} 3 \mathrm{~b}$, and subsequent activation of the terminal complement pathway with concurrent formation of C5a and C5b-9 (also known as the membrane attack complex, MAC) (11). Both the anaphylatoxins C3a and $\mathrm{C} 5 \mathrm{a}$, by acting on their respective receptors, and the (sublytic) MAC have been shown to induce inflammatory responses (12-16). Local and/or systemic low-grade inflammation (LGI) is believed to play a major role in the development of insulin resistance in several organs (17-20), and C3, or the complement system in general, may also contribute to this process.

Several cross-sectional studies have shown associations of plasma C3 levels with insulin resistance, measured by homeostasis model assessment (HOMA-IR) or hyperinsulinaemic euglycaemic clamp studies, and with type 2 diabetes mellitus (T2DM) (21-23). However, prospective or longitudinal studies on the relation between C3 and the progression of insulin resistance and/or the incidence of T2DM are scarce. To the best of our knowledge, there are no longitudinal data on the relation between (changes in) C3 levels and (changes in) IR. One prospective cohort study showed a positive association of serum C3 with incident T2DM, but this cohort consisted of only adult men (24) and data on women are not yet available. Moreover, the mechanisms behind this observed prospective association are still unclear.

For these reasons, we explored the relation of plasma C3 levels with progression of insulin resistance and glucose tolerance, as well as with incidence of T2DM in a prospective cohort of Caucasian subjects with 7-year follow-up and an extensive (metabolic) characterization of participants, the CODAM study (Cohort on Diabetes and Atherosclerosis Maastricht) $(6,25)$. This cohort is particularly suitable to address these issues, as it consists mainly of subjects selected for an increased risk of metabolic diseases, and reasonable progression of IR and glucose parameters could therefore be expected within the 7 years' follow-up. Within the present study, we first investigated the longitudinal associations between (changes in) C3 levels with (changes in) whole body (HOMA2-IR) and organ-specific (liver, adipocyte) insulin resistance and three measures of glucose tolerance (i.e. fasting glucose, $2 \mathrm{~h}$ glucose, and $\mathrm{AUC}_{\text {glucose }}$ ) during the 7-year follow-up. Second, we examined whether plasma C3 levels at baseline were associated with incident T2DM. 


\section{Research design and methods}

\section{Subjects and study design}

The Cohort on Diabetes and Atherosclerosis Maastricht (CODAM) study is a prospective, observational study on, among others, the natural progression of IR and glucose tolerance. A total of 574 individuals were selected from a large populationbased cohort as described in detail elsewhere $(6,25)$, and were extensively characterized at baseline with regard to their lifestyle, and cardiovascular and metabolic profile during two visits to the University's metabolic research unit. After a median of 7.0 years [IQR 6.9-7.1], 495 subjects participated in the follow-up measurements (attrition rate 14\% - Figure 4.3.1). The CODAM study was approved by the Medical Ethical Committee of the Maastricht University Medical Centre, and all subjects gave written informed consent.

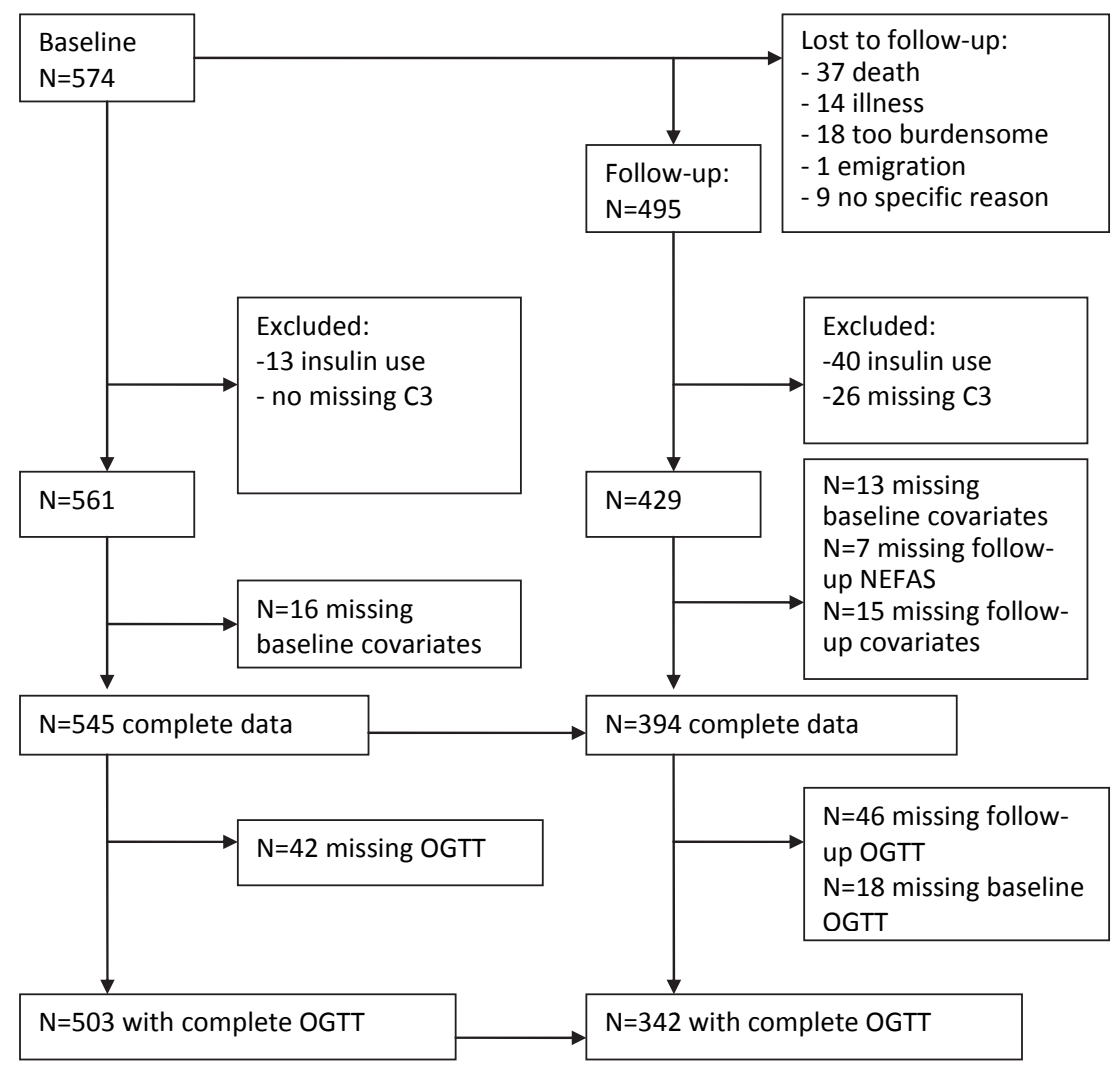

Figure 4.3.1. Flow chart of study participants. 
For the present study, we excluded participants on insulin therapy ( $n=13$ at baseline, $\mathrm{n}=40$ at follow-up). Baseline data were complete in 545 subjects, except for data derived from the oral glucose tolerance test (OGTT), which were complete in 503 subjects. Follow-up data were complete in 394 subjects, of whom 342 also had complete OGTT data at follow-up. Generally, the 545 included participants were healthier than the 29 excluded subjects, as indicated by lower adiposity measures, lower glucose, insulin and fatty acid levels, and lower IR indices at baseline in included participants (data not shown). Similarly, the 394 subjects included in the analyses with follow-up data were healthier at baseline than the 180 subjects without or with incomplete follow-up data, as indicated by lower adiposity measures, lower glucose, insulin, and fatty acid levels, and lower use of anti-hypertensive, lipid-lowering, and glucose-lowering medication at baseline in these subjects (data not shown).

Some of the biomarkers that were obtained in the CODAM study, i.e. C3, insulin, and the inflammatory markers (interleukin-6 (IL-6), high sensitivity C-reactive protein (hs-CRP), serum amyloid A (SAA), and soluble inter-cellular adhesion molecule- 1 (sICAM-1)) were measured at the time the baseline evaluation of the CODAM study was completed $(6,25)$. More recently, paired (re-)measurements of these biomarkers at baseline and follow-up were performed, but with different methods. To make use of all available data and to ensure comparability of baseline and follow-up values, we calculated the mean of both baseline measurements after calibration of previous to recent baseline measurements using Deming regression models, which is recommended for method comparison (26), as described before (27).

\section{Complement factor 3 at baseline and at follow-up}

Participants were asked to stop their lipid-lowering drugs 14 days before the visit, and to stop all other medication the day before the visit. After an overnight fast, venous blood samples were collected for assessment of biomarkers. Serum was allowed to clot at room temperature for $45 \mathrm{~min}$, and after centrifugation at $3000 \mathrm{rpm}$ for $15 \mathrm{~min}$, serum aliquots were stored at $-20^{\circ} \mathrm{C}$, and plasma (EDTA) aliquots were stored at $-80^{\circ} \mathrm{C}$ until use. Samples were thawed only once prior to measurements. At baseline, C3 levels were determined in serum by an automatic analyzer (Hitachi 912) with a Roche kit (Roche Diagnostics Netherlands BV) - inter-assay coefficient of variation of $2.1 \%$. Paired measurements of plasma C3 levels at baseline and follow-up were also performed using the IMMAGE immunochemistry system C3 assay (Beckman-Coulter) inter-assay coefficient of variation of $7 \%$. For baseline C3 values, the mean of the two C3 measurements (after calibration) was used for further analyses.

\section{Insulin resistance and glucose tolerance at baseline and at follow-up}

A standard 75g OGTT was performed with measurement of glucose levels at 0, 30, 60, and $120 \mathrm{~min}$, and, during the follow-up round, also at $15 \mathrm{~min}$. At follow-up, a second 
measurement of fasting glucose was also obtained from a second visit, and the mean of these fasting glucose levels was used for further analyses. All plasma glucose levels were measured in NaF/KOx plasma with a hexokinase glucose-6 phosphate dehydrogenase method (ABX Diagnostics) with inter-assay coefficient of variation (CV) $<5 \%$.

Fasting insulin concentrations at baseline were determined in EDTA plasma using a two-sided immunoradiometric test (IRMA) using paired monoclonal antibodies (Medgenix Diagnostics) with inter-assay CV of $6.0 \%(6,25)$. Paired measurements of plasma insulin levels were performed at baseline and follow-up, at all OGTT time-points mentioned above, using a multi-array (MA) detection system based on electrochemiluminicence technology (SECTOR Imager 2400, Meso Scale Discovery, Gaithersburg, MD, USA) with inter-assay CV of 9.7\%. For fasting insulin at baseline, the mean of the two measurements (after calibration) was used for analyses. Fasting nonesterified free fatty acids (NEFAs) at baseline were measured in EDTA plasma using an enzymatic calorimetric NEFA C method (Wako Diagnostics, Richmond, USA) with inter-assay $\mathrm{CV}<5 \%$. In addition, paired measurements of NEFAs at baseline and follow-up were performed using the same method. At baseline, the mean of the two measurements was taken for analyses.

Total area under the curves (AUC) for glucose and insulin during the OGTT (for the first 30 minutes and for 120 minutes) were calculated using the trapezoidal method. HOMA2-IR, which has been shown to correlate well with (muscle) glucose disposal in clamp studies (28), was computed using the HOMA2-calculator considering fasting plasma glucose and specific insulin data (http://www.dtu.ox.ac.uk/homacalculator/ index.php). Hepatic insulin resistance (hepatic IR) was estimated using the square root of the product of the areas under the curve for glucose and insulin during the first 30 minutes of the OGTT (i.e. SQRT(glucose ${ }_{0-30}[\mathrm{AUC}] \mathrm{x}$ insulin $_{0-30}$ [AUC])) converted to conventional units $([\mathrm{mg} / \mathrm{dl} \cdot \mathrm{hr}] \times[\mathrm{ulU} / \mathrm{ml} \cdot \mathrm{hr}])(29,30)$. This index has been developed and validated against the product of fasting plasma insulin and endogenous glucose production in clamp studies, and has been postulated to represent hepatic insulin resistance, as it not only takes into account fasting glucose, but also the early suppression of fasting glucose by insulin during the first phase of the OGTT. Adipocyte insulin resistance (adipocyte IR) was calculated as the product of fasting insulin and fasting NEFA concentrations, as described before $(25,31,32)$.

\section{Other covariates}

Body mass index, waist circumference, smoking behaviour, family history of T2DM (first-degree relatives), dietary calorie intake, mean daily alcohol consumption, physical activity, and use of anti-hypertensive, glucose-lowering, and lipid-lowering medication were determined at baseline and follow-up as previously described $(6,25)$. Interleukin- 6 (IL-6), high sensitivity C-reactive protein (hs-CRP), serum amyloid A (SAA), and soluble inter-cellular adhesion molecule-1 (SICAM-1) were determined at baseline using 
methods described before (6). In addition, paired measurements of baseline and follow-up IL-6, hs-CRP, SAA, sICAM-1, interleukin-8 (IL-8), and tumour necrosis factor- $\alpha$ (TNF- $\alpha$ ) were determined in EDTA on a multi-array detection system based on electrochemiluminescence technology (SECTOR Imager 2400, Meso Scale Discovery, USA) $(27,33)$. At baseline, when applicable, the mean of two measurements was used for analyses after calibration. Glomerular filtration rate (eGFR) and prior cardiovascular disease (CVD) at baseline, and glucose metabolism status [i.e. normal (NGM), impaired (IGM), or T2DM] both at baseline and follow-up, were determined as previously described $(6,34,35)$.

\section{Statistical analysis}

Variables with a skewed distribution (i.e. HOMA2-IR, hepatic IR, adipocyte IR, fasting glucose, postprandial ( $2 \mathrm{~h}$ ) glucose, $\mathrm{AUC}_{\text {glucose, }}$ all inflammatory markers) were In transformed prior to further analyses. A low-grade inflammation (LGI) score was calculated by averaging the Z-scores [i.e. (individual's observed values - population mean)/SD] of the six (In transformed) inflammatory markers (IL-6, IL-8, TNF- $\alpha$, hs-CRP, SAA, sICAM-1) $(6,25,33)$, with the population mean and SD for each individual circulating biomarker based on its average of the baseline and follow-up measurements.

We performed three different analyses to examine the association of plasma C3 levels with insulin resistance and glucose tolerance. First, we investigated the overall longitudinal associations of $\mathrm{C} 3$ levels with insulin resistance and glucose tolerance over the 7-year period in all subjects $(n=545)$ with generalized estimating equations (GEE) with an exchangeable correlation structure to account for the correlation of repeated measurements within subjects (36). Because outcome variables were In-transformed, regression coefficients were expressed in \% change (e.g. \% increase in HOMA2-IR per $0.1 \mathrm{~g} / \mathrm{l}$ increase in C3). Second, we investigated whether changes in plasma C3 levels were associated with (absolute) changes in insulin resistance and glucose tolerance during the 7-year follow-up in subjects with follow-up data $(n=394)$ using linear regression. As changes in insulin resistance and glucose tolerance were normally distributed, the regression coefficients were expressed as changes in outcome variables (e.g. units increase in the change in HOMA2-IR per $0.1 \mathrm{~g} / \mathrm{l}$ increase in the change in C3). Finally, we investigated whether baseline plasma C3 levels were associated with 7-year incidence of T2DM in the subjects without T2DM at baseline $(n=333)$ using logistic regression analysis.

All analyses were first adjusted for (changes in) age, sex, follow-up time (GEE analysis only), glucose metabolism status (insulin resistance outcomes only) [models 1]. Next, additional adjustments were done for prior CVD (baseline only), eGFR (baseline only), smoking status, alcohol consumption, dietary energy intake, physical activity, family history of T2DM, and use of medication [models 2]. Subsequently, (changes in) waist circumference [models 3] and the LGI score [models 4] were added in separate 
models, since their relation with $\mathrm{C} 3$ is not straightforward. For instance, obesity may be considered a simple confounder, but may also represent an ascending proxy (4-6) or intermediate factor/mediator in the causal path between C3 and insulin resistance (37), in which case its addition to the regression models would lead to overadjustment and hence to underestimation of the true effect sizes (38). Vice versa, LGI may be considered an intermediate factor between C3 and insulin resistance, but may also represent an ascending proxy $(39,40)$, or even act as a simple confounder. Because of the observational nature of our data, we are unable to disentangle whether waist circumference and LGI are simple confounders, whether they are ascending proxies, or mediators. We also investigated whether the associations differed between sexes by adding interaction terms between $\mathrm{C} 3$ and sex to the (fully adjusted) models. We found no such effect modification by sex and therefore all results are presented for men and women combined. A two-sided $p$-value of $<0.05$ was considered statistically significant. All analyses were performed using STATA Data Analysis and Statistical Software for Windows, version 9.0/SE (STATACorp LP, Texas, USA).

\section{Results}

\section{Study population}

Table 4.3.1 shows characteristics of the whole study population at baseline $(n=545)$ and follow-up $(n=394)$. Generally, most variables remained unchanged over the 7-year follow-up period. Indices of insulin resistance showed a small decrease over time, while $2 \mathrm{~h}$ glucose and AUC glucose and markers of LGI seemed to increase over time. The mean plasma C3 level increased over time from $1.01 \mathrm{~g} / \mathrm{l}$ to $1.14 \mathrm{~g} / \mathrm{l}$, while adiposity measures remained relatively stable.

\section{Associations of plasma C3 levels with whole-body and organ-specific insulin resistance and glucose tolerance over the 7-year follow-up period}

We examined the overall association of plasma C3 levels with insulin resistance and glucose tolerance using GEE analyses ( $n=545$ at baseline, of which $n=394$ also at followup). Over the 7-year follow-up period, plasma C3 levels (per $0.1 \mathrm{~g} / \mathrm{l}$ ) were longitudinally associated with HOMA2-IR, hepatic IR, and adipocyte IR after adjustments for age, sex, follow-up time, and glucose metabolism status (Table 4.3.2; model 1), and remained significant after subsequent adjustment for potential confounders (model 2): $\beta=15.2 \%$ (95\% Cl 12.9-17.6) for HOMA2-IR, $\beta=6.1 \%(95 \% \mathrm{Cl} 4.7-7.4)$ for hepatic IR, and $\beta=16.0 \%$ (95\% Cl 13.0-19.1) for adipocyte IR. Plasma C3 levels (per $0.1 \mathrm{~g} / \mathrm{l}$ ) were also longitudinally associated with fasting glucose $(\beta=1.8 \%[95 \% \mathrm{Cl} 1.2-2.4]), 2 \mathrm{~h}$ glucose $\left(\beta=5.2\right.$ [95\% Cl 3.7-6.7]), and $A \cup C_{\text {glucose }}(\beta=3.6 \%[95 \% \mathrm{Cl} 2.7-4.6])$ (model2). When these associations were further adjusted for) waist circumference (model 3 ) and finally the 
LGI score (model 4), these positive associations were attenuated, but remained statistically significant: $\beta=8.7 \%(95 \% \mathrm{Cl} 6.5-11.0)$ for HOMA2-IR, $\beta=3.9 \%(95 \% \mathrm{Cl} 2.4-5.3)$ for hepatic IR, $\beta=8.0 \%(95 \% \mathrm{Cl} 5.3-10.9)$ for adipocyte IR, $\beta=0.8 \%(95 \% \mathrm{Cl} 0.2-1.5)$ for fasting glucose, $\beta=3.5 \%(95 \% \mathrm{Cl} 1.9-5.2)$ for $2 \mathrm{~h}$ glucose), and $\beta=2.2 \%(95 \% \mathrm{Cl} 1.2-3.2)$ for $A \cup C_{\text {glucose. }}$

Table 4.3.1 Characteristics of the study population at baseline and follow-up.

\begin{tabular}{|c|c|c|c|}
\hline & \multirow{2}{*}{$\begin{array}{c}\mathrm{N}=545 \\
\text { Baseline }\end{array}$} & \multicolumn{2}{|c|}{ N=394 with follow-up data } \\
\hline & & Baseline & Follow-up \\
\hline Age (years) & $59 \pm 7.0$ & $59 \pm 7.0$ & $66 \pm 6.9$ \\
\hline Male sex (\%) & 62 & 60 & 60 \\
\hline NGM/IGM/T2DM (\%) & $54 / 22 / 24$ & $60 / 25 / 15$ & $46 / 25 / 29$ \\
\hline Prior CVD (\%) & 28 & 25 & n.a. \\
\hline Current smoker (\%) & 22 & 22 & 16 \\
\hline Family history of T2DM (\%) & 43 & 43 & 49 \\
\hline Energy intake $\left(10^{3} \bullet \mathrm{kcal} / \mathrm{d}\right)$ & $2.22 \pm 0.67$ & $2.25 \pm 0.66$ & $2.12 \pm 0.62$ \\
\hline Alcohol (g/d) & $8.62[1.38-22.68]$ & $9.15[2.04-22.5]$ & $8.69[1.48-20.0]$ \\
\hline Physical activity $\left(10^{3} \bullet\right.$ METs/wk) & $6.66 \pm 4.23$ & $6.67 \pm 4.21$ & $7.14 \pm 4.60$ \\
\hline \multicolumn{4}{|l|}{ Use of medication } \\
\hline Antihypertensive (\%) & 38 & 33 & 55 \\
\hline Lipid-lowering (\%) & 18 & 17 & 40 \\
\hline Glucose-lowering (\%) & 11 & 6 & 18 \\
\hline Body mass index $\left(\mathrm{kg} / \mathrm{m}^{2}\right)$ & $28.5 \pm 4.3$ & $28.2 \pm 4.1$ & $28.3 \pm 4.2$ \\
\hline Waist circumference $(\mathrm{cm})$ & $99.2 \pm 11.9$ & $98.0 \pm 11.5$ & $99.6 \pm 11.9$ \\
\hline Fasting Triglycerides (mmol/l) & $1.40[1.00-2.00]$ & $1.40[1.00-1.90]$ & $1.40[1.10-1.90]$ \\
\hline Fasting glucose (mmol/l) & $5.6[5.2-6.3]$ & $5.5[5.1-6.0]$ & $5.3[5.0-5.9]$ \\
\hline Fasting insulin (pmol/l) & $74[50-114]$ & $69[49-100]$ & 66 [47-99] \\
\hline Fasting NEFAs $(\mu \mathrm{mol} / \mathrm{l})$ & $528 \pm 185$ & $519 \pm 177$ & $459 \pm 171$ \\
\hline $2 \mathrm{~h}$ glucose $(\mathrm{mmol} / \mathrm{l})^{*}$ & $6.7[5.3-9.4]$ & $6.5[5.2-8.6]$ & 7.8 [5.9-10.7] \\
\hline AUC glucose $(\mathrm{mmol} / / \bullet \mathrm{min})^{*}$ & 1010 [860-1237] & 973 [832-1186] & 1046 [882-1264] \\
\hline HOMA2-IR & 1.62 [1.10-2.53] & $1.51[1.07-2.17]$ & $1.44[1.02-2.16]$ \\
\hline Hepatic IR index* & $39[32-50]$ & 38 [31-48] & $35[28-44]$ \\
\hline Adipocyte IR index & $36[22-62]$ & $33[22-54]$ & 28 [19-43] \\
\hline $\mathrm{C} 3(\mathrm{~g} / \mathrm{l})$ & $1.01 \pm 0.16$ & $1.01 \pm 0.15$ & $1.14 \pm 0.19$ \\
\hline IL-6 (pg/ml) & $1.56[1.13-2.27]$ & $1.51[1.10-2.20]$ & $1.53[1.07-2.36]$ \\
\hline IL-8 (pg/ml) & $4.36[3.59-5.51]$ & $4.27[3.50-5.35]$ & $5.07[4.20-6.63]$ \\
\hline TNF- $\alpha(\mathrm{pg} / \mathrm{ml})$ & $6.23[5.27-7.56]$ & $6.17[5.27-7.40]$ & $6.55[5.54-8.26]$ \\
\hline $\mathrm{hs}-\mathrm{CRP}(\mathrm{mg} / \mathrm{l})$ & 2.07 [0.97-3.96] & $1.87[0.91-3.73]$ & 1.97 [0.93-3.89] \\
\hline SAA (mg/l) & $1.44[0.99-2.26]$ & $1.42[0.99-2.24]$ & $1.47[0.88-2.72]$ \\
\hline sICAM-1 ( $\mu \mathrm{g} / \mathrm{l})$ & 213 [187-245] & 208 [185-242] & $213[188-250]$ \\
\hline LGI score & $-0.07 \pm 0.65$ & $-0.14 \pm 0.62$ & $0.03 \pm 0.72$ \\
\hline eGFR $\left(\mathrm{ml} / \mathrm{min} / 1.73 \mathrm{~m}^{2}\right)$ & $86 \pm 17$ & $85 \pm 16$ & - \\
\hline
\end{tabular}

Data are expressed as mean $\pm \mathrm{SD}$, median [interquartile range] or percentages; NGM: normal glucose metabolism; IGM, impaired glucose metabolism; T2DM: type 2 diabetes mellitus; CVD: cardiovascular disease; NEFAs: non-esterified fatty acids; AUC glucose: area under the curve for glucose during $75 \mathrm{~g}$ oral glucose tolerance test; HOMA2-IR: homeostasis model assessment insulin resistance; Hepatic IR index: hepatic insulin resistance index; Adipocyte IR: adipocyte insulin resistance index; C3: complement factor 3; IL-6: interleukin-6; IL-8: interleukin-8; TNF- $\alpha$ : tumour necrosis factor- $\alpha$; hs-CRP: high sensitivity C-reactive protein; SAA: serum amyloid A; sICAM-1: soluble inter-cellular adhesion molecule-1; LGI score: low-grade inflammation score; eGFR: estimated glomerular filtration rate; n.a.: not available. ${ }^{*}$ Variables derived from oral glucose tolerance tests (OGTT) were complete in $n=503$ at baseline and $n=342$ at follow-up. 


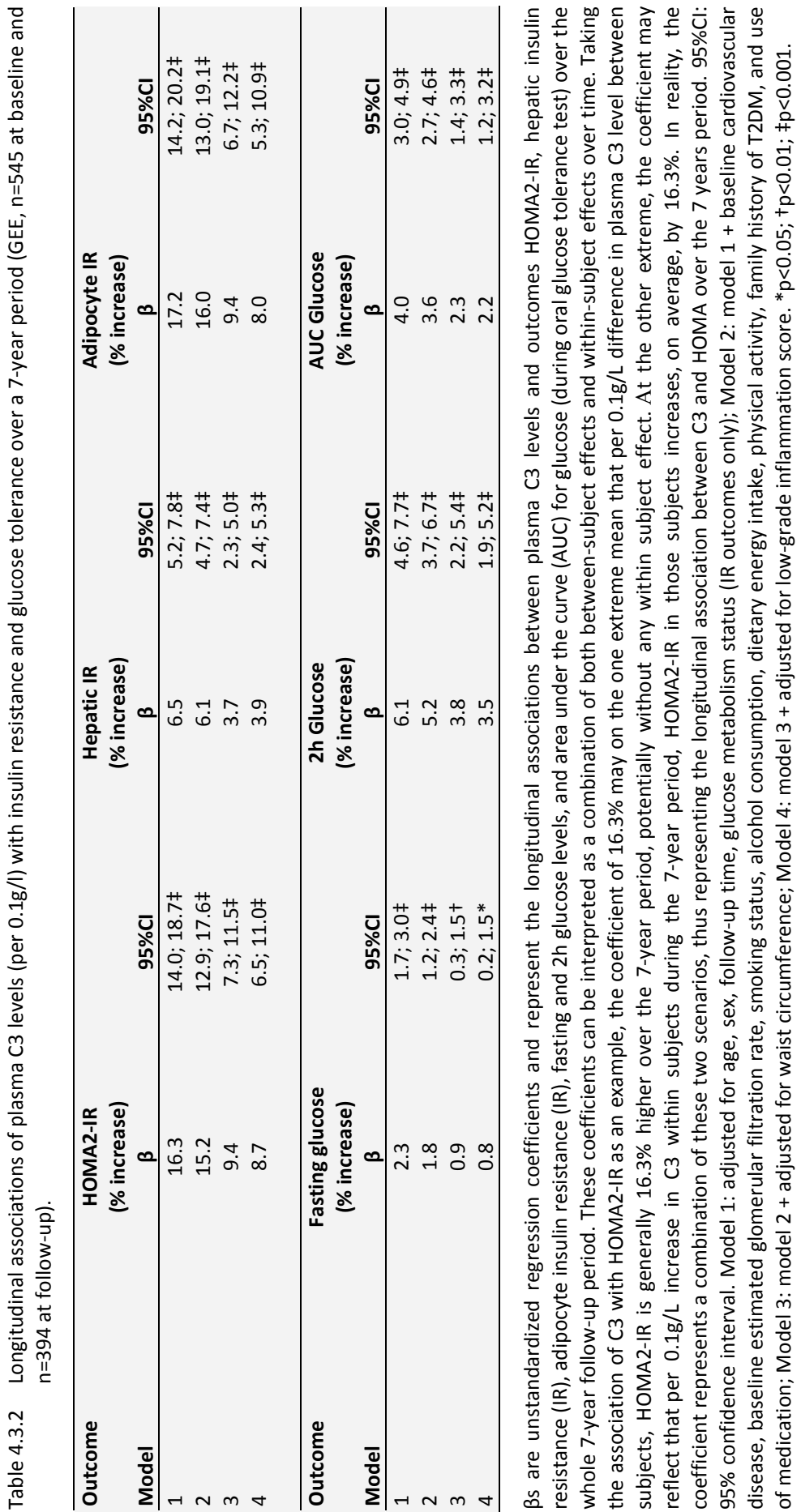




\section{Associations of changes in plasma C3 levels with changes in whole-body and organ-specific insulin resistance and changes in glucose tolerance}

The coefficients of GEE analysis can be interpreted as a combination of both between-subject effects and within-subject effects over time without distinguishing between the two. In other words, and taking the association of C3 with HOMA2-IR as an example, the coefficient of $15.2 \%$ may on the one extreme mean that per $0.1 \mathrm{~g} / \mathrm{l}$ difference in plasma C3 level between subjects, HOMA2-IR is generally $15.2 \%$ higher over the 7-year period, potentially without any within-subject effect. At the other extreme, the coefficient may reflect that per $0.1 \mathrm{~g} / \mathrm{l}$ increase in $\mathrm{C} 3$ within subjects during the 7-year period, HOMA2-IR in those subjects increases, on average, by $15.2 \%$.

To address specifically the within-subject component of these associations, we next investigated whether changes in C3 levels were associated with changes in insulin resistance and glucose tolerance in the 394 subjects with baseline and available follow-up data. During the 7-year follow-up, greater changes in C3 levels (per $0.1 \mathrm{~g} / \mathrm{l}$ ) were positively associated with changes in HOMA2-IR $(\beta=0.18[95 \% \mathrm{Cl} 0.12-0.24])$, changes in hepatic IR $(\beta=1.31[95 \% \mathrm{Cl} 0.64-1.98])$, and changes in adipocyte IR $(\beta=3.23$ [95\% Cl 1.09-5.37]) after adjustment for changes in age, sex, and glucose metabolism status (Table 4.3.3; model 1). These associations, again, remained unchanged after further adjustment for changes in potential confounders (model 2). The magnitude of these effects in units was comparable to the magnitude of the effects of GEE models, which were reported in \% (e.g. using the median HOMA2-IR of 1.51 at baseline, a $15.2 \%$ increase for HOMA2-IR (model 2) corresponds to an absolute change of 0.23 units in HOMA2-IR). Further adjustment for changes in waist circumference (model 3 ), and finally changes in the LGI score (model 4), resulted in attenuation, but the associations of changes in C3 with changes in HOMA2-IR $(\beta=0.08$ [95\% Cl 0.02-0.15]) and changes in hepatic IR $(\beta=0.87[95 \% \mathrm{Cl} 0.12-1.61])$ did remain statistically significant. Finally, no statistically significant associations were observed between changes in C3 levels and changes in fasting glucose, $2 \mathrm{~h}$ glucose, or $\mathrm{AUC}_{\text {glucose }}$ during the 7 -year follow-up. 


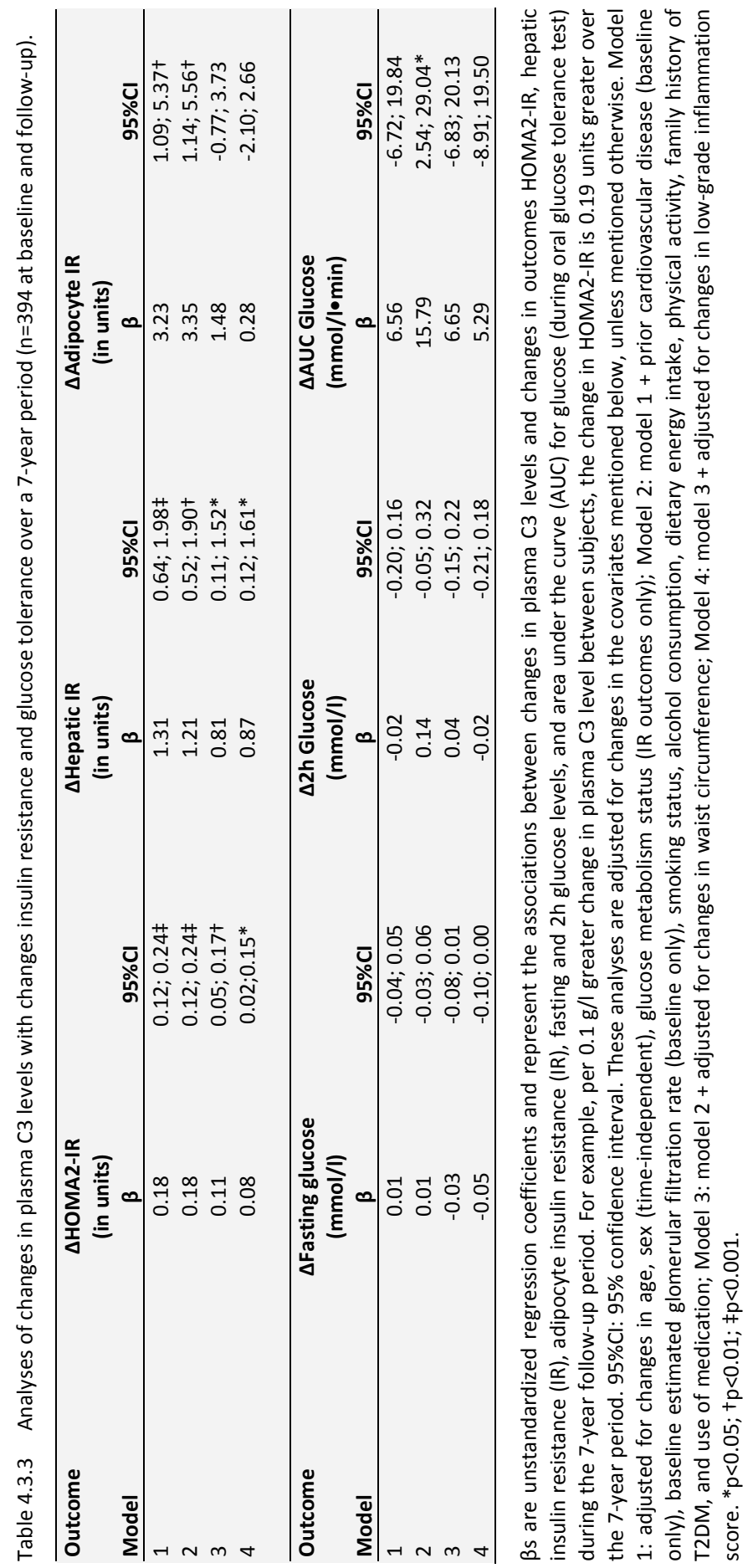




\section{Association of baseline plasma C3 levels with incident T2DM during the 7-year follow-up}

After subjects with T2DM at baseline were excluded from the analysis $(n=61)$, 333 subjects were available for logistic regression analysis on incident T2DM. During the 7-year follow-up, 57 out of 333 subjects developed T2DM (17\%). Baseline plasma C3 levels (per $0.1 \mathrm{~g} / \mathrm{l}$ ) were associated with a higher risk of developing T2DM (OR=1.6 [95\% Cl 1.3-2.0]) after adjustment for baseline age and sex (Table 4.3.4; model 1). Also, after adjustment for prior CVD, estimated glomerular filtration rate (baseline only), smoking status, alcohol consumption, dietary energy intake, physical activity, family history of T2DM, use of medication (model 2), waist circumference (model 3), and the LGI score (model 4), baseline C3 remained independently associated with incident T2DM (OR=1.5 [95\% Cl 1.1-2.0]). Additional adjustment for baseline HOMA2-IR did also not change this effect size (OR=1.5 [95\% Cl 1.1-2.0]).

Table 4.3.4 Associations of baseline plasma C3 levels with incident type 2 diabetes mellitus (T2DM) over a 7-year period in subjects without diabetes at baseline (logistic regression analysis, $n=333$ ).

\begin{tabular}{lccc}
\hline Outcome & Model & OR & 95\%Cl \\
\hline Incident T2DM & 1 & 1.6 & $1.3 ; 2.0 \ddagger$ \\
& 2 & 1.6 & $1.2 ; 2.0 \ddagger$ \\
& 3 & 1.5 & $1.1 ; 1.9 \dagger$ \\
\hline
\end{tabular}

Odds ratios (OR) represent the associations between baseline plasma C3 levels and incident type 2 diabetes mellitus (T2DM) at 7-year follow-up. Per $0.1 \mathrm{~g} / \mathrm{l}$ higher plasma C3 level at baseline, the odds to develop incident T2DM during 7-year follow-up are 1.6 times higher. 95\% Cl: 95\% confidence interval. Model 1: adjusted for baseline age, sex; Model 2: model 1 + prior cardiovascular disease, estimated glomerular filtration rate (baseline only), smoking status, alcohol consumption, dietary energy intake, physical activity, family history of T2DM, and use of medication; Model 3: model 2 + adjusted for baseline waist circumference; Model 4: model $3+$ adjusted for baseline low-grade inflammation score. ${ }^{*} p<0.05 ; \uparrow p<0.01 ; \neq p<0.001$.

\section{Additional analyses}

To account for the possibility that $\mathrm{C} 3$ levels were affected by the presence of infectious or inflammatory diseases at the time of blood sampling, we also repeated all analyses excluding subjects with hs-CRP levels $>10 \mathrm{mg} / \mathrm{l}(\mathrm{n}=37$ at baseline, $\mathrm{n}=41$ at follow-up). This did not materially change the associations of C3 with metabolic outcomes (data not shown). In addition, similar associations were observed after stringent exclusion of subjects with any self-reported (history of) pulmonary, renal, gastro-intestinal, thyroid, or rheumatic disease, or cancer at baseline ( $n=200$, data not shown). Finally, C3 levels may also have been influenced by female sex hormones $(41,42)$. However, exclusion of pre-menopausal women (21\%) and women using hormone replacement therapy (9\%) at baseline did not influence or findings (total excluded $n=63$, data not shown). 


\section{Discussion}

In this prospective cohort study we showed that, during a 7-year follow-up, baseline levels of $\mathrm{C3}$, the central complement component, were independently associated with incident T2DM in both men and women, and that plasma C3 levels were independently associated with insulin resistance in muscle, liver, and adipocytes, as well as with glucose tolerance. Moreover, changes in C3 levels were associated with changes in muscle IR, hepatic IR, and adipocyte IR (although the latter not independently of changes in obesity), but not with changes in glucose tolerance.

To the best of our knowledge, this is the first longitudinal study on the association of circulating C3 with detailed measures of insulin resistance as well as glucose tolerance. Our observations extend the previously reported association of plasma C3 with incident T2DM in a prospective cohort study in men (24) by showing that this association was not only present in men, but also in women. The effect size observed in our study (OR 1.51 per $0.1 \mathrm{~g} / \mathrm{l}$ ) was somewhat larger than the effect reported by Engström et al. (OR 1.37 per $0.2 \mathrm{~g} / \mathrm{l}$ ), potentially related to a selection of subjects with increased risk of cardiometabolic diseases in the present study $(6,25)$. The 7 -year incidence of T2DM in CODAM was $17 \%$, which was substantially higher than the 6-year incidence of $4.5 \%$ reported by Engström for the cohort of men participating in a screening program (24). Both studies adjusted for a comparable set of potential confounders, with our present study also adjusting for six common markers of LGI. Importantly, our change analyses showed that within-subject changes in C3 levels are also associated with changes in each of the three measures of insulin resistance used, but not with changes in glucose tolerance. Altogether, these findings suggest that plasma C3 levels may underlie progression of insulin resistance, and eventually may lead to T2DM.

In the change analyses, we observed an attenuation of effect sizes after adjustment for waist circumference, particularly for adipocyte IR (Table 4.3.3; model 3). This may be interpreted as simple confounding, since abdominal obesity is a known determinant of both insulin resistance and plasma C3 levels $(4,5,43,44)$. However, abdominal obesity may also be considered an ascending proxy in the causal pathway between C3 and insulin resistance, particularly insulin resistance in muscle (HOMA2-IR). Adjustments for an ascending proxy are not always necessary, as they can attenuate estimates due to differences in bias and variance between waist circumference and C3 (38). Moreover, it has also been suggested that C3 may actually precede obesity (37), in which case changes in obesity should be considered a mediator, for which adjustment is also not necessary. Likewise, although LGI may be considered an intermediate factor and/or an ascending proxy in the pathway between C3 and insulin resistance, we could not exclude the possibility that LGI represents a simple confounder, as proinflammatory cytokines that cause insulin resistance $(19,20)$, may also increase C3 levels $(39,40)$. Therefore, the true effect sizes may actually lie in between models 2 and 3/4, as these 
latter models may have been underestimations due to unnecessary adjustment for waist circumference and LGI. Nevertheless, the associations of changes in C3 with changes in HOMA2-IR and hepatic IR remained statistically significant in all models.

The observed associations of C3 levels with insulin resistance over time and with incident T2DM can be explained by at least two pathophysiological mechanisms that may occur simultaneously. First, higher plasma levels of C3 may be associated with more complement activation, and higher levels of its activated product $\mathrm{C} 3 \mathrm{a}$ as well as the terminal pathways activation products $\mathrm{C} 5 \mathrm{a}$ and $\mathrm{sC5b}-9$. The anaphylatoxins $\mathrm{C} 3 \mathrm{a}$ and C5a, by acting on their respective receptors which are highly expressed in adipose tissue and Kupffer cells in the liver (45-47), attract and activate leucocytes, and increase the synthesis of pro-inflammatory cytokines by several cell types, including leucocytes and Kupffer cells $(12-16,47,48)$. Such local and/or systemic inflammation is believed to play a major role in the development of insulin resistance in several organs (17-20). Indeed, blocking of anaphylatoxic pathways in C3aR- or C5aR-knockout mice fed a high fat diet led to decreased adipocyte size, less liver steatosis, less adipose tissue infiltration by macrophages, a reduction in tissue and plasma pro-inflammatory cytokines, and less systemic insulin resistance $(45,48)$, and inhibition of C3a and C5a signalling by blocking their receptors appeared to induce similar effects (47). As such, complement activation and the subsequent local and/or systemic inflammation may actively contribute to the development of insulin resistance and T2DM. In agreement, we observed a modest attenuation of the observed associations of C3 with HOMA2-IR and adipocyte IR after adjustment for the LGI score (models 4), which could be interpreted as mediation. However, this mediating effect might have been underestimated, since our LGI score did not include IL- $\beta$, which is an important proinflammatory cytokine that is up-regulated upon inflammasome activation by C3a and (sublytic) MAC (12-14).

Second, C3 may not by itself contribute actively to the development of insulin resistance, but rather serve as a marker of adipocyte dysfunction. Adipocytes have been shown to produce C3, and activate it to C3a and finally C3a-desarg, also known as acylation stimulating protein (ASP), which promotes the storage of glucose and lipids in adipocytes in a way similar to insulin (49-51). Because C3 levels also correlate with postprandial triglyceride levels in vivo $(23,52)$, it was hypothesized that high levels of C3 are a marker of high postprandial triglycerides, for example due to ASP resistance. ASP resistance, which may be initiated by fatty acids or the pro-inflammatory cytokine TNF- $\alpha(53,54)$, would then result in a redistribution of glucose and lipids to other organs, leading to insulin resistance in liver and muscle $(23,40,51,52)$. Indeed, in mouse models, interruption of the ASP system led to decreased triglyceride storage in adipose tissue, delayed lipid clearance, impaired glucose tolerance, and insulin resistance $(55,56)$. Data in humans are, however, not yet available.

A major strength of the present longitudinal cohort study is the comprehensive set of analyses on the association of C3 with detailed measures of both insulin resistance 
and glucose tolerance at baseline and 7-year follow-up, adjusted for major (potential) confounders. As for any longitudinal study, this set of analyses is limited by the fact that not all subjects attended the follow-up evaluation, and our effect sizes may have been underestimated because of selective attrition (unhealthy subjects not attending follow-up) (57). Also, despite the availability of longitudinal data, the observational nature of our study does not allow us to draw definite conclusions on causality, and therefore we cannot fully exclude a reverse causation scenario. Insulin may have several effects on C3 transcription and production (58), and a potential inhibitory effect of insulin on C3 levels may be lost in an insulin resistant state. On the other hand, several hyperinsulinaemic clamp studies did not observe changes in adipose tissue C3 mRNA expression or serum C3 levels $(8,22)$. Finally, our study consisted of middle-aged and older, Caucasian subjects who were selected on the basis of an increased risk for metabolic and CVD, and extrapolation to other study populations or other ethnicities should be done with caution. However, this does not impair the value of our cohort for aetiological research, and our study population represents a large group of subjects present in the general population, in whom several metabolic risk factors, including C3, may contribute to the development of cardiovascular and metabolic diseases over time.

In conclusion, we have shown that baseline C3 levels were independently associated with incident T2DM in men and women at 7-year follow-up. In addition, (changes in) C3 levels were strongly associated with (changes in) the degree of insulin resistance in muscle, liver and adipocytes. Changes in plasma C3 levels may therefore reflect a progression of metabolic dysregulation, including progression of low-grade inflammation and insulin resistance, that eventually leads to abnormalities in glucose tolerance and frank T2DM. Moreover, higher C3 levels may be associated with complement activation in liver and adipose tissue. Whether C3 itself and/or downstream complement activation products can causally contribute to the development of insulin resistance and T2DM, remains to be investigated in humans. 


\section{References}

1. Onat A, Hergenc G, Can G, Kaya Z, Yuksel H: Serum complement C3: a determinant of cardiometabolic risk, additive to the metabolic syndrome, in middle-aged population. Metabolism 2010;59:628-634

2. Muscari A, Bozzoli C, Puddu GM, Sangiorgi Z, Dormi A, Rovinetti C, Descovich GC, Puddu P: Association of serum C3 levels with the risk of myocardial infarction. Am J Med 1995;98:357-364

3. Hertle E, van Greevenbroek MM, Stehouwer CD: Complement C3: an emerging risk factor in cardiometabolic disease. Diabetologia 2012;55:881-884

4. Hernandez-Mijares A, Jarabo-Bueno MM, Lopez-Ruiz A, Sola-Izquierdo E, Morillas-Arino C, MartinezTriguero $\mathrm{ML}$ : Levels of $\mathrm{C} 3$ in patients with severe, morbid and extreme obesity: its relationship to insulin resistance and different cardiovascular risk factors. Int J Obes (Lond) 2007;31:927-932

5. Warnberg J, Nova E, Moreno LA, Romeo J, Mesana MI, Ruiz JR, Ortega FB, Sjostrom M, Bueno M, Marcos A: Inflammatory proteins are related to total and abdominal adiposity in a healthy adolescent population: the AVENA Study. Am J Clin Nutr 2006;84:505-512

6. Wlazlo N, van Greevenbroek MM, Ferreira I, Jansen EJ, Feskens EJ, van der Kallen CJ, Schalkwijk CG, Bravenboer B, Stehouwer CD: Low-grade inflammation and insulin resistance independently explain substantial parts of the association between body fat and serum C3: The CODAM study. Metabolism 2012;61:1787-1796

7. Alper CA, Johnson AM, Birtch AG, Moore FD: Human C'3: evidence for the liver as the primary site of synthesis. Science 1969;163:286-288

8. Koistinen HA, Vidal H, Karonen SL, Dusserre E, Vallier P, Koivisto VA, Ebeling P: Plasma acylation stimulating protein concentration and subcutaneous adipose tissue C3 mRNA expression in nondiabetic and type 2 diabetic men. Arterioscler Thromb Vasc Biol 2001;21:1034-1039

9. Choy LN, Rosen BS, Spiegelman BM: Adipsin and an endogenous pathway of complement from adipose cells. J Biol Chem 1992;267:12736-12741

10. Naughton MA, Botto M, Carter MJ, Alexander GJ, Goldman JM, Walport MJ: Extrahepatic secreted complement C3 contributes to circulating C3 levels in humans. J Immunol 1996;156:3051-3056

11. Walport MJ: Complement. First of two parts. N Engl J Med 2001;344:1058-1066

12. Asgari E, Le Friec G, Yamamoto H, Perucha E, Sacks SS, Kohl J, Cook HT, Kemper C: C3a modulates IL1beta secretion in human monocytes by regulating ATP efflux and subsequent NLRP3 inflammasome activation. Blood 2013; DOI 10.1182/blood-2013-05-502229

13. Triantafilou K, Hughes TR, Triantafilou M, Morgan BP: The complement membrane attack complex triggers intracellular Ca2+ fluxes leading to NLRP3 inflammasome activation. J Cell Sci 2013;126: 2903-2913

14. Laudisi F, Spreafico R, Evrard M, Hughes TR, Mandriani B, Kandasamy M, Morgan BP, Sivasankar B, Mortellaro A: Cutting Edge: The NLRP3 Inflammasome Links Complement-Mediated Inflammation and IL-1beta Release. J Immunol 2013;191:1006-1010

15. Takabayashi T, Vannier E, Clark BD, Margolis NH, Dinarello CA, Burke JF, Gelfand JA: A new biologic role for C3a and C3a desArg: regulation of TNF-alpha and IL-1 beta synthesis. J Immunol 1996;156: 3455-3460

16. Fischer WH, Jagels MA, Hugli TE: Regulation of IL-6 synthesis in human peripheral blood mononuclear cells by C3a and C3a(desArg). J Immunol 1999;162:453-459

17. Xu H, Barnes GT, Yang Q, Tan G, Yang D, Chou CJ, Sole J, Nichols A, Ross JS, Tartaglia LA, Chen H: Chronic inflammation in fat plays a crucial role in the development of obesity-related insulin resistance. J Clin Invest 2003;112:1821-1830

18. Chen J, Wildman RP, Hamm LL, Muntner P, Reynolds K, Whelton PK, He J: Association between inflammation and insulin resistance in U.S. nondiabetic adults: results from the Third National Health and Nutrition Examination Survey. Diabetes Care 2004;27:2960-2965

19. van Greevenbroek MM, Schalkwijk CG, Stehouwer CD: Obesity-associated low-grade inflammation in type 2 diabetes mellitus: causes and consequences. Neth J Med 2013;71:174-187

20. Krogh-Madsen R, Plomgaard P, Moller K, Mittendorfer B, Pedersen BK: Influence of TNF-alpha and IL-6 infusions on insulin sensitivity and expression of IL-18 in humans. Am J Physiol Endocrinol Metab 2006;291:E108-114 
21. Muscari A, Antonelli S, Bianchi G, Cavrini G, Dapporto S, Ligabue A, Ludovico C, Magalotti D, Poggiopollini G, Zoli M: Serum C3 is a stronger inflammatory marker of insulin resistance than Creactive protein, leukocyte count, and erythrocyte sedimentation rate: comparison study in an elderly population. Diabetes Care 2007;30:2362-2368

22. Weyer C, Tataranni PA, Pratley RE: Insulin action and insulinemia are closely related to the fasting complement C3, but not acylation stimulating protein concentration. Diabetes Care 2000;23:779-785

23. van Oostrom AJ, Alipour A, Plokker TW, Sniderman AD, Cabezas MC: The metabolic syndrome in relation to complement component 3 and postprandial lipemia in patients from an outpatient lipid clinic and healthy volunteers. Atherosclerosis 2007;190:167-173

24. Engstrom G, Hedblad B, Eriksson KF, Janzon L, Lindgarde F: Complement C3 is a risk factor for the development of diabetes: a population-based cohort study. Diabetes 2005;54:570-575

25. Wlazlo N, van Greevenbroek MM, Ferreira I, Jansen EH, Feskens EJ, van der Kallen CJ, Schalkwijk CG, Bravenboer B, Stehouwer CD: Iron metabolism is associated with adipocyte insulin resistance and plasma adiponectin: the Cohort on Diabetes and Atherosclerosis Maastricht (CODAM) study. Diabetes Care 2013;36:309-315

26. Martin RF: General deming regression for estimating systematic bias and its confidence interval in method-comparison studies. Clin Chem 2000;46:100-104

27. van Bussel BC, Ferreira I, van de Waarenburg MP, van Greevenbroek MM, van der Kallen CJ, Henry RM, Feskens EJ, Stehouwer CD, Schalkwijk CG: Multiple inflammatory biomarker detection in a prospective cohort study: a cross-validation between well-established single-biomarker techniques and an electrochemiluminescense-based multi-array platform. PLoS One 2013;8:e58576; DOI 10.1371/journal.pone.0058576

28. Wallace TM, Levy JC, Matthews DR: Use and abuse of HOMA modeling. Diabetes Care 2004;27: 1487-1495

29. Abdul-Ghani MA, Matsuda M, Balas B, DeFronzo RA: Muscle and liver insulin resistance indexes derived from the oral glucose tolerance test. Diabetes Care 2007;30:89-94

30. Vangipurapu J, Stancakova A, Kuulasmaa T, Paananen J, Kuusisto J, Ferrannini E, Laakso M: A novel surrogate index for hepatic insulin resistance. Diabetologia 2011;54:540-543

31. Vangipurapu J, Stancakova A, Pihlajamaki J, Kuulasmaa TM, Kuulasmaa T, Paananen J, Kuusisto J, Ferrannini E, Laakso M: Association of indices of liver and adipocyte insulin resistance with 19 confirmed susceptibility loci for type 2 diabetes in 6,733 non-diabetic Finnish men. Diabetologia 2011;54:563-571

32. Abdul-Ghani MA, Molina-Carrion M, Jani R, Jenkinson C, Defronzo RA: Adipocytes in subjects with impaired fasting glucose and impaired glucose tolerance are resistant to the anti-lipolytic effect of insulin. Acta Diabetol 2008;45:147-150

33. van Bussel BC, Henry RM, Schalkwijk CG, Ferreira I, Feskens EJ, Streppel MT, Smulders YM, Twisk JW, Stehouwer CD: Fish consumption in healthy adults is associated with decreased circulating biomarkers of endothelial dysfunction and inflammation during a 6-year follow-up. J Nutr 2011;141:1719-1725

34. Du $H$, van der AD, van Bakel MM, van der Kallen CJ, Blaak EE, van Greevenbroek MM, Jansen EH, Nijpels G, Stehouwer CD, Dekker JM, Feskens EJ: Glycemic index and glycemic load in relation to food and nutrient intake and metabolic risk factors in a Dutch population. Am J Clin Nutr 2008;87:655-661

35. Levey AS, Bosch JP, Lewis JB, Greene T, Rogers N, Roth D: A more accurate method to estimate glomerular filtration rate from serum creatinine: a new prediction equation. Modification of Diet in Renal Disease Study Group. Ann Intern Med 1999;130:461-470

36. Twisk JWR: Applied longitudinal data analysis for epidemiology: a practical guide. Cambridge, Cambridge University Press, 2003

37. Engstrom G, Hedblad B, Janzon L, Lindgarde F: Weight gain in relation to plasma levels of complement factor 3: results from a population-based cohort study. Diabetologia 2005;48:2525-2531

38. Schisterman EF, Cole SR, Platt RW: Overadjustment bias and unnecessary adjustment in epidemiologic studies. Epidemiology 2009;20:488-495

39. Volanakis JE: Transcriptional regulation of complement genes. Annu Rev Immunol 1995;13:277-305

40. Kalant D, Maslowska M, Scantlebury T, Wang H, Cianflone K: Control of lipogenesis in adipose tissue and the role of acylation stimulating protein. Can J Diab 2003;27:154-171 
41. Li SH, Huang HL, Chen YH: Ovarian steroid-regulated synthesis and secretion of complement C3 and factor $B$ in mouse endometrium during the natural estrous cycle and pregnancy period. Biol Reprod 2002;66:322-332

42. Yilmazer M, Fenkci V, Fenkci S, Aktepe O, Sonmezer M, Kurtay G: Association of serum complement $(\mathrm{C} 3, \mathrm{C} 4)$ and immunoglobulin (IgG, IgM) levels with hormone replacement therapy in healthy postmenopausal women. Hum Reprod 2003;18:1531-1535

43. Pomeroy C, Mitchell J, Eckert E, Raymond N, Crosby R, Dalmasso AP: Effect of body weight and caloric restriction on serum complement proteins, including Factor D/adipsin: studies in anorexia nervosa and obesity. Clin Exp Immunol 1997;108:507-515

44. Hernandez-Mijares A, Banuls C, Bellod L, Jover A, Sola E, Morillas C, Victor VM, Rocha M: Effect of weight loss on C3 and C4 components of complement in obese patients. Eur J Clin Invest 2012;42: 503-509

45. Mamane Y, Chung Chan C, Lavallee G, Morin N, Xu L, Huang J, Gordon R, Thomas W, Lamb J, Schadt EE, Kennedy BP, Mancini JA: The C3a anaphylatoxin receptor is a key mediator of insulin resistance and functions by modulating adipose tissue macrophage infiltration and activation. Diabetes 2009;58: 2006-2017

46. Schlaf G, Schieferdecker HL, Rothermel E, Jungermann K, Gotze O: Differential expression of the C5a receptor on the main cell types of rat liver as demonstrated with a novel monoclonal antibody and by C5a anaphylatoxin-induced Ca2+ release. Lab Invest 1999;79:1287-1297

47. Lim J, Iyer A, Suen JY, Seow V, Reid RC, Brown L, Fairlie DP: C5aR and C3aR antagonists each inhibit dietinduced obesity, metabolic dysfunction, and adipocyte and macrophage signaling. FASEB J 2013;27:822-831

48. Phieler J, Chung KJ, Chatzigeorgiou A, Klotzsche-von Ameln A, Garcia-Martin R, Sprott D, Moisidou M, Tzanavari T, Ludwig B, Baraban E, Ehrhart-Bornstein M, Bornstein SR, Mziaut H, Solimena M, Karalis KP, Economopoulou M, Lambris JD, Chavakis T: The Complement Anaphylatoxin C5a Receptor Contributes to Obese Adipose Tissue Inflammation and Insulin Resistance. J Immunol 2013;

49. Saleh J, Summers LK, Cianflone K, Fielding BA, Sniderman AD, Frayn KN: Coordinated release of acylation stimulating protein (ASP) and triacylglycerol clearance by human adipose tissue in vivo in the postprandial period. J Lipid Res 1998;39:884-891

50. Germinario R, Sniderman AD, Manuel S, Lefebvre SP, Baldo A, Cianflone K: Coordinate regulation of triacylglycerol synthesis and glucose transport by acylation-stimulating protein. Metabolism 1993;42:574-580

51. Cianflone K, Xia Z, Chen LY: Critical review of acylation-stimulating protein physiology in humans and rodents. Biochim Biophys Acta 2003;1609:127-143

52. Halkes CJ, van Dijk H, de Jaegere PP, Plokker HW, van Der Helm Y, Erkelens DW, Castro Cabezas M: Postprandial increase of complement component 3 in normolipidemic patients with coronary artery disease: effects of expanded-dose simvastatin. Arterioscler Thromb Vasc Biol 2001;21:1526-1530

53. Wen Y, Wang H, Maclaren R, Wu J, Lu H, Cianflone K: Palmitate and oleate induction of acylation stimulating protein resistance in 3T3-L1 adipocytes and preadipocytes. J Cell Biochem 2008;104: 391-401

54. MacLaren R, Kalant D, Cianflone K: The ASP receptor C5L2 is regulated by metabolic hormones associated with insulin resistance. Biochem Cell Biol 2007;85:11-21

55. Cui W, Paglialunga S, Kalant D, Lu H, Roy C, Laplante M, Deshaies Y, Cianflone K: Acylation-stimulating protein/C5L2-neutralizing antibodies alter triglyceride metabolism in vitro and in vivo. Am J Physiol Endocrinol Metab 2007;293:E1482-1491

56. Paglialunga S, Schrauwen P, Roy C, Moonen-Kornips E, Lu H, Hesselink MK, Deshaies Y, Richard D, Cianflone K: Reduced adipose tissue triglyceride synthesis and increased muscle fatty acid oxidation in C5L2 knockout mice. J Endocrinol 2007;194:293-304

57. Weuve J, Tchetgen Tchetgen EJ, Glymour MM, Beck TL, Aggarwal NT, Wilson RS, Evans DA, Mendes de Leon CF: Accounting for bias due to selective attrition: the example of smoking and cognitive decline. Epidemiology 2012;23:119-128

58. Wang P, Keijer J, Bunschoten A, Bouwman F, Renes J, Mariman E: Insulin modulates the secretion of proteins from mature 3T3-L1 adipocytes: a role for transcriptional regulation of processing. Diabetologia 2006;49:2453-2462 


\section{Chapter 4.4}

Activated complement factor 3 is associated with

liver fat and liver enzymes

Nick Wlazlo, Marleen MJ van Greevenbroek, Isabel Ferreira, Eugene HJM Jansen, Edith JM Feskens, Carla JH van der Kallen, Casper G Schalkwijk, Bert Bravenboer, Coen DA Stehouwer 


\section{Abstract}

\section{Objective}

The complement system may be involved in the pathogenesis of alcoholic and non-alcoholic liver disease, though studies in humans are scarce. For this reason, we investigated whether circulating levels of activated complement factor 3 (C3a) were associated with hepatic steatosis and hepatocellular damage.

\section{Materials and methods}

Plasma C3a, aspartate aminotransferase (AST), alanine aminotransferase (ALT) and gamma-glutamyl transferase (GGT) were determined in 523 individuals (61\% men, age $59 \pm 7$ years). Liver enzymes were standardized and compiled into a liver enzyme score. Liver fat content was estimated using a predictive equation that has recently been validated with magnetic resonance spectrometry. Cross-sectional associations between C3a and liver fat or liver enzymes were investigated with multiple linear regression analyses, stratified in no-to-moderate versus heavy alcohol consumers (men: $>30 \mathrm{~g} /$ day; women: $>20 \mathrm{~g} /$ day).

\section{Results}

C3a was associated with liver fat percentage both in the no-to-moderate $(\beta=0.223$; $95 \% \mathrm{Cl} 0.036-0.409)$ and the heavy alcohol consumers $(\beta=0.632 ; 95 \% \mathrm{Cl} 0.259-1.004$; P-interaction=0.047). C3a was also associated with the liver enzyme score in heavy alcohol consumers $(\beta=0.917 ; 95 \% \mathrm{Cl} 0.443-1.392)$, but not in no-to-moderate alcohol consumers $(\beta=0.042 ; 95 \% \mathrm{Cl}-0.198$ to $0.281 ;$ P-interaction $=0.001)$.

\section{Conclusions}

C3a levels, as a marker of complement activation, were associated with liver fat content and hepatocellular injury, at least in subjects who consume considerable amounts of alcohol daily. 


\section{Introduction}

Alcoholic and non-alcoholic fatty liver disease (AFLD and NAFLD respectively) represent the two most common causes of liver disease in western countries $(1,2)$. They both consist of a spectrum ranging from simple hepatic steatosis, to steatohepatitis, liver fibrosis and cirrhosis $(3,4)$. There is growing evidence that chronic inflammation and activation of the immune system contributes to the development and progression of both diseases, with a key role for tumour necrosis factor alpha (TNF- $\alpha$ ) produced by Kupffer cells $(4,5)$. The complement system is part of the innate and adaptive immune systems, and has an important function in the response to tissue injury and infection (6). Activation of the complement system through any one of the three activation pathways results in cleavage of complement factor 3 (C3) into $\mathrm{C} 3 \mathrm{~b}$ and the anaphylotoxin C3a (6), which is responsible for attracting and activating leukocytes, in particular granulocytes and monocytes, to local inflammatory sites (7). The complement system can be activated by apoptosis and by bacterial lipopolysacharide (LPS) (6), the concentration of which is increased in patients with AFLD due to increased intestinal permeability (8), but also by (chronic) alcohol exposure itself $(9,10)$. Interestingly, animal studies have shown that receptors for C3a (C3aR) are highly expressed on Kupffer cells in the liver (11), and by acting on these receptors, C3a has been shown to stimulate prostaglandin, TNF- $\alpha$, and interleukin- 6 (IL- 6 ) secretion by Kupffer cells $(12,13)$.

For this reason, activation of the complement system has been implicated in the pathogenesis of AFLD and NAFLD. Deposition of complement factors C1, C3 and C8 was increased in livers of several animal models of AFLD (14-16). Moreover, C3 knockout mice were protected from alcohol-induced liver steatosis and hepatocyte injury, as measured by serum alanine aminotransferase (ALT) $(10,17)$. In addition, C3aR and C5aR knockout mice, or mice that were depleted of liver macrophages (i.e. Kupffer cell depleted), all lacked alcohol-induced increases in hepatic TNF- $\alpha$ (15), suggesting that C3a- and C5a-signalling on Kupffer cells may contribute to TNF- $\alpha$ production and subsequent liver damage. For NAFLD, there is also some evidence for a role of complement activation. In C3aR knockout mice on a high fat diet, liver triglycerides and plasma ALT were lower compared with wild-type mice, and were accompanied by improved insulin sensitivity (11). Furthermore, C3b deposition was abundant in liver biopsies in morbidly obese humans with non-alcoholic steatohepatitis (NASH), but not in healthy controls (18), and plasma levels of C3a were higher in a small sample of patients with NAFLD / NASH than in healthy controls (19).

In view of these considerations, activation of the complement system seems to be involved in both AFLD and NAFLD, but human studies on this subject are scarce $(18,19)$, especially for alcoholic liver disease. Also, human studies usually concern associations of complement factors with obesity and/or insulin resistance (19-22), of which NAFLD is a serious consequence, but less often focus particularly on aspects of fatty liver disease 
itself. For these reasons, we specifically investigated whether plasma C3a levels, as a marker of complement activation by any of the three pathways, were associated with hepatic steatosis and markers of hepatocellular injury, independently of its known association with obesity. In particular, we explored whether these associations were different for AFLD and NAFLD, which we discriminated using the amount of daily alcohol consumption. In addition, we examined whether associations between C3a and liver fat or liver enzymes were explained (i.e. mediated) by the proinflammatory cytokine TNF- $\alpha$ or insulin resistance, given the anaphylotoxic function of C3a, and the effect of C3aR signalling on insulin sensitivity described above (11).

\section{Materials and methods}

\section{Subjects and study design}

The Cohort on Diabetes and Atherosclerosis Maastricht (CODAM) is a prospective cohort study of subjects with (an increased risk of) type 2 diabetes mellitus (T2DM) and cardiovascular diseases (CVD). Study participants were selected from a large cohort in the general population as described in detail elsewhere $(20,23)$. In total, 574 individuals were included and extensively characterized with regard to their lifestyle, cardiovascular and metabolic profile during two visits to the University's metabolic research unit.

The current cross-sectional analyses were performed in the baseline evaluation of this cohort and excluded participants on insulin therapy $(n=13)$, with self-reported (history of) liver disease $(n=7)$, or with missing data on liver enzymes $(n=8)$, C3a $(n=2)$, or important covariates $(n=21)$. Hence, the present study reports on 523 subjects. The study protocol was approved by the Medical Ethical Committee of the University of Maastricht and the University Hospital Maastricht, and all subjects gave written informed consent.

\section{Measurements of C3a}

Participants were asked to stop their lipid-lowering drugs 14 days before the visit, and to stop all other medication the day before the visit. After an overnight fast, venous blood samples were collected in pre-cooled EDTA vacutainer tubes for assessment of several biomarkers, and an oral glucose tolerance test (OGTT) was performed. After centrifugation at $3000 \mathrm{rpm}$ for $15 \mathrm{~min}$ at $4^{\circ} \mathrm{C}$, plasma aliquots were stored at $-80^{\circ} \mathrm{C}$ until use. Samples were thawed only once prior to measurements. C3a levels were determined in EDTA plasma by ELISA (MicroVue C3a plus EIA kit, Quidel). The interassay coefficient of variation was $6.62 \%$. 


\section{Liver enzymes, inflammatory markers and insulin resistance}

Aspartate amino transferase (AST), alanine aminotransferase (ALT), and gamma-glutamyl transferase (GGT) were measured in EDTA plasma using conventional clinical chemistry methods. TNF $\alpha$ was determined in EDTA plasma on a multi-array detection system based on electro-chemiluminicence technology (SECTOR Imager 2400, Meso Scale Discovery, USA) with an interassay CV of 5.8\%. High sensitivity C-reactive protein (hs-CRP) was measured in serum as previously described (20). Fasting plasma glucose and insulin concentrations were determined as previously described (20), and insulin resistance (IR) was estimated using the homeostasis model assessment (HOMA2-IR) calculator (http://www.dtu.ox.ac.uk/homacalculator/index.php).

\section{Other covariates}

BMI and waist circumference (WC) were measured as previously described $(20,21)$. Smoking behaviour (yes/no) was assessed by questionnaire. Mean alcohol consumption was estimated by a validated food frequency questionnaire and expressed in grams per day (24). Alcohol consumption was considered "none" if subjects consumed less than $10 \mathrm{~g}$ alcohol per year $(<0.028 \mathrm{~g} / \mathrm{d})$. Moderate alcohol consumption was defined as $0.028-30 \mathrm{~g} / \mathrm{d}$ for men, and $0.028-20 \mathrm{~g} / \mathrm{d}$ for women $(3,23)$. Heavy alcohol consumption was defined as $>30 \mathrm{~g} / \mathrm{d}$ for men, and $>20 \mathrm{~g} / \mathrm{d}$ for women. Serum triglycerides and nonesterified fatty acids (NEFAs) were determined as described before (23). Complement C3 levels were measured in serum by an automatic analyzer (Hitachi 912) with a Roche kit (Roche Diagnostics Netherlands BV) with an interassay CV of 2.14\% $(20,21)$. Creatinine levels were determined in EDTA plasma with a Jaffé diagnostic test (Roche Diagnostics), and glomerular filtration rate was estimated using the short Modification of Diet in Renal Disease (MDRD) equation (25). Glucose metabolism status was diagnosed according to the WHO 1999 criteria as previously described (20). Presence of the metabolic syndrome was defined according to the definition of the International Diabetes Federation (IDF) (26). Prior CVD was diagnosed as previously described in detail (20).

\section{Statistical analysis}

Variables with a skewed distribution (i.e. C3a, all liver enzymes, HOMA2-IR, triglycerides, TNF- $\alpha$, hs-CRP) were In transformed prior to further analyses. We estimated liver fat content (eLF\%) with the use of a predictive equation that has recently been developed and validated against proton magnetic resonance spectroscopy $\left({ }^{1} \mathrm{H}-\mathrm{MRS}\right)$, and provides an inexpensive tool for epidemiological research (27). This equation estimates liver fat content on the basis of data on liver enzymes (AST and AST/ALT ratio), fasting insulin, presence of the metabolic syndrome (IDF definition) and presence of T2DM (area under receiver operating curve of 0.86; $95 \% \mathrm{Cl}$ 0.80-0.92). A combined liver enzyme score (LE score) was calculated as follows: 
first, all three liver enzymes were In transformed to obtain a normal distribution. Next, the population mean and standard deviation were calculated for each In transformed liver enzyme. Then, for each individual, these In liver enzymes were transformed into Z-scores (i.e. standardized values) by subtracting this population mean from the individuals' observed value and dividing by the standard deviation (i.e. individuals' observed value - population mean) / SD). Finally, for each individual the three standardized liver enzymes were averaged to obtain a single, robust measure of hepatocellular injury for that individual, to be used for linear regression analyses. This composite score was used to provide a robust measure of hepatocellular injury, as it can be interpreted as three repeated measurements of the same construct, thereby reducing measurement error. The use of such a score also reduces the influence of biological variability of each marker if assessed separately, and prevents multiple testing problems.

General characteristics of the study population were compared across tertiles of plasma C3a levels using analysis of variance (ANOVA) for continuous variables and x2-test for discrete variables. The associations of (In-transformed) C3a with the estimated liver fat percentage, the liver enzyme score, and individual liver enzymes were examined with the use of multiple linear regression analyses. In order to examine whether the association of C3a with liver outcomes differed according to the levels of alcohol consumption, we conducted stratified analyses in "no-to-moderate alcohol consumers" ( $n=411)$ and "heavy alcohol consumers" ( $n=112)$ defined using sex-specific cut-offs for alcohol consumption $>30 \mathrm{~g} /$ day for men and $>20 \mathrm{~g} /$ day for women $(3,28)$. Differences in the strength of the associations between these groups were tested by adding interaction terms between $\mathrm{C} 3 \mathrm{a}$ and the level of alcohol consumption to the linear regression models. Since only a small number of subjects was classified as "no alcohol consumer" ( $n=48)$, we did not analyse "no" and "moderate" alcohol consumers separately.

In addition, we examined the mediating role of TNF- $\alpha$ in the associations (if present) between $\mathrm{C} 3 \mathrm{a}$ and estimated liver fat content and the liver enzyme score. The mediating role of HOMA2-IR was only investigated in the associations (if present) of C3a with the liver enzyme score, as insulin was also used in the equation to estimate liver fat content. The magnitude of mediation was calculated by quantifying the extent to which the regression coefficient of the association between C3a and liver fat content or the liver enzyme score was attenuated by adding a mediator (i.e. TNF- $\alpha$ or HOMA2-IR) to the regression model. Statistical significance of such a mediated 'effect' by TNF- $\alpha$ or HOMA2-IR was assessed by calculating $95 \%$ confidence intervals around the magnitude of attenuation in regression coefficients, with the use of bootstrapping (5000 samples) using the SPSS macro by Preacher and Hayes (29).

We also investigated whether the associations between C3a and liver outcomes differed between sexes by adding interaction terms to (the fully adjusted) models. We found no such effect-modification by sex and therefore all results are presented for 
men and women combined. A two-sided p-value of $<0.05$ was considered statistically significant. All analyses were performed using the Statistical Package for Social Sciences (SPSS) for Windows, version 17.0 (SPSS Inc., Chicago, IL, USA).

\section{Results}

\section{Study population}

Characteristics of the overall study population according to tertiles of plasma C3a are shown in Table 4.4.1. Subjects in higher C3a tertiles generally had a worse metabolic and cardiovascular profile, with an increasing prevalence of CVD and increasing HOMA2-IR across C3a tertiles. Estimated liver fat percentage increased linearly with increasing levels of $\mathrm{C} 3 \mathrm{a}$, but liver enzymes and the combined liver enzyme score were not different across $\mathrm{C} 3 \mathrm{a}$ tertiles in the overall population. Median alcohol consumption was $4.7 \mathrm{~g} / \mathrm{d}$ (IQR 0.6-12.2) in the no-to-moderate alcohol consumers and $40.1 \mathrm{~g} / \mathrm{d}$ (IQR 32.1-50.) in the heavy alcohol consumers $(p=<0.001)$. Median C3a levels were 59.8 $\mathrm{ng} / \mathrm{ml}$ (IQR 50.1-73.0) in no-to-moderate alcohol consumers and $56.9 \mathrm{ng} / \mathrm{ml}$ (IQR 49.4$71.5)$ in heavy alcohol consumers $(p=0.209)$.

\section{Association of C3a with estimated liver fat}

After adjustment for age, sex, CVD, eGFR, smoking status, alcohol use, and medication, C3a was significantly associated with estimated liver fat percentage both in the no-to-moderate $(\beta=0.223 ; 95 \% \mathrm{Cl} 0.036-0.409)$ and the heavy alcohol consumers $(\beta=0.632 ; 95 \% \mathrm{Cl} 0.259-1.004)$. The association was stronger in the latter (Table 4.4.2, model 2; P-interaction=0.047). Adjustment for waist circumference attenuated these associations to $\beta=0.071(95 \% \mathrm{Cl}-0.085-0.228)$ and $\beta=0.336(95 \% \mathrm{Cl}-0.002-0.674$; $\mathrm{P}$-interaction $=0.091$ ) respectively. Similar attenuation was observed after adjustment for C3, the precursor protein of C3a (Table 4.4.2; model 2b).

Next, we investigated whether the association between $\mathrm{C} 3 \mathrm{a}$ and estimated liver fat percentage in the heavy alcohol consumers $(\beta=0.336 ; 95 \% \mathrm{Cl}-0.002-0.674$; model 2a) was mediated by TNF- $\alpha$. Adjustment for TNF- $\alpha$ attenuated the association of C3a with liver fat content to $\beta=0.237(95 \% \mathrm{Cl}-0.108-0.582)$, resulting in a magnitude of mediation by TNF- $\alpha$ of $\beta=0.099(95 \% \mathrm{Cl} 0.005-0.269)$. As such, TNF- $\alpha$ explained $29 \%$ of the association between $\mathrm{C} 3 \mathrm{a}$ and liver fat. 
Table 4.4.1 General characteristics of the study population ( $n=523$ ) according to tertiles of C3a.

\begin{tabular}{|c|c|c|c|c|}
\hline & & C3a tertiles & & \\
\hline & Lowest ( $n=174)$ & Middle ( $n=175)$ & Highest (n=174) & p \\
\hline Age (years) & $59.5 \pm 6.5$ & $59.2 \pm 7.3$ & $59.7 \pm 7.1$ & 0.852 \\
\hline Male sex (\%) & 68 & 64 & 51 & 0.003 \\
\hline NGM/IGM/T2DM (\%) & $59 / 20 / 21$ & $53 / 25 / 22$ & $48 / 24 / 28$ & 0.279 \\
\hline Metabolic syndrome (\%) & 49 & 59 & 67 & 0.002 \\
\hline CVD (\%) & 20 & 30 & 33 & 0.013 \\
\hline Current smoker (\%) & 20 & 22 & 20 & 0.873 \\
\hline Alcohol consumption*: & & & & \\
\hline none/moderate/heavy (\%) & $5 / 70 / 25$ & $9 / 71 / 20$ & $14 / 67 / 19$ & 0.039 \\
\hline Use of medication & & & & \\
\hline Antihypertensive (\%) & 28 & 42 & 44 & 0.004 \\
\hline Lipid-lowering (\%) & 13 & 18 & 24 & 0.045 \\
\hline Glucose-lowering (\%) & 11 & 13 & 10 & 0.687 \\
\hline Body mass index $(\mathrm{kg} / \mathrm{m} 2)$ & $27.5 \pm 3.6$ & $28.2 \pm 4.3$ & $29.7 \pm 4.5$ & $<0.001$ \\
\hline Waist circumference $(\mathrm{cm})$ & & & & \\
\hline Men & $99.1 \pm 9.7$ & $101.4 \pm 9.3$ & $106.4 \pm 12.9$ & $<0.001$ \\
\hline Women & $92.7 \pm 10.5$ & $92.6 \pm 11.9$ & $97.7 \pm 11.9$ & 0.01 \\
\hline Complement C3 (g/l) & $1.67 \pm 0.31$ & $1.81 \pm 0.30$ & $1.92 \pm 0.35$ & $<0.001$ \\
\hline C3a (ng/ml) & 46.1 [40.6 - 49.9] & $59.2[56.2-63.2]$ & $81.2[72.6-97.1]$ & $<0.001$ \\
\hline Glucose (mmol/l) & $5.6[5.2-6.3]$ & $5.6[5.2-6.1]$ & $5.5[5.2-6.5]$ & 0.541 \\
\hline Insulin (pmol/l) & $52[41-70]$ & $62[45-86]$ & $67[48-106]$ & $<0.001$ \\
\hline HOMA2-IR & $1.02[0.79-1.37]$ & 1.22 [0.85 - 1.69] & $1.30[0.91-2.02]$ & $<0.001$ \\
\hline Triglycerides (mmol/l) & $1.30[1.00-1.93]$ & $1.50[1.00-2.00]$ & 1.40 [1.00 - 1.93] & 0.787 \\
\hline NEFAs (mmol/l) & $0.52 \pm 0.18$ & $0.52 \pm 0.18$ & $0.53 \pm 0.18$ & 0.821 \\
\hline eLF\% (\%) & $3.62[2.05-5.92]$ & $4.84[2.50-7.66]$ & $5.43[2.50-8.24]$ & $<0.001$ \\
\hline $\operatorname{AST}(U / I)+$ & 19.5 [15.9 - 24.0] & 20.2 [16.6 - 24.9] & $19.8[16.3-23.8]$ & 0.503 \\
\hline $\operatorname{ALT}(U / I)+$ & 21.4 [17.1 - 27.0] & 23.4 [17.4 - 29.6] & 21.7 [16.9 - 27.7] & 0.337 \\
\hline GGT $(U / I)+$ & $24[16-37]$ & $24[18-37]$ & 24 [18 - 39] & 0.728 \\
\hline Liver enzyme score ${ }^{\dagger}$ & $-0.06 \pm 0.83$ & $0.03 \pm 0.83$ & $0.03 \pm 0.91$ & 0.479 \\
\hline TNF- $\alpha(p g / m l)$ & $6.06[4.90-7.35]$ & $6.30[5.42-7.61]$ & $6.32[5.34-7.91]$ & 0.063 \\
\hline $\mathrm{hs}-\mathrm{CRP}(\mathrm{mg} / \mathrm{l})$ & $1.78[1.08-3.14]$ & 2.82 [1.49 - 4.69] & $3.52[2.12-7.05]$ & $<0.001$ \\
\hline eGFR & $86 \pm 15$ & $87 \pm 18$ & $85 \pm 18$ & 0.470 \\
\hline
\end{tabular}

Data are expressed as mean $\pm \mathrm{SD}$, median [interquartile range] or percentages; the minimum and maximum of C3a tertiles were [25.6-52.4], [52.5-67.3] and [67.4-212.4] ng/mL respectively; NGM: normal glucose metabolism; IGM, impaired glucose metabolism; T2DM: type 2 diabetes mellitus; CVD: cardiovascular disease; HOMA2-IR: homeostasis model assessment insulin resistance; NEFA's: non-esterified fatty acids; eLF\%: estimated liver fat percentage; AST: aspartate amino transferase; ALT: alanine aminotransferase; GGT: gamma-glutamyl transferase; TNF- $\alpha$ : tumour necrosis factor- $\alpha$; hs-CRP: high sensitivity C-reactive protein; eGFR: estimated glomerular filtration rate. * Alcohol consumption was considered "none" if subjects consumed $<0.028 \mathrm{~g} / \mathrm{d}$. Moderate alcohol consumption was defined as $0.028-30 \mathrm{~g} / \mathrm{d}$ for men, and 0.028-20 g/d for women. Heavy alcohol consumption was defined as $>30 \mathrm{~g} / \mathrm{d}$ for men, and $>20 \mathrm{~g} / \mathrm{d}$ for women. † Liver enzymes and the score were not different across C3a tertiles in the overall population, but did increase significantly across C3a tertiles in the group of heavy drinkers (see also Figure 4.4.1). 
Table 4.4.2 Associations of C3a with liver fat and liver enzymes stratified by alcohol consumption (sexspecific cut-offs $>30 \mathrm{~g} / \mathrm{d}$ for men, and $>20 \mathrm{~g} / \mathrm{d}$ for women).

\begin{tabular}{|c|c|c|c|c|c|c|c|}
\hline \multirow[b]{2}{*}{ Outcome } & \multirow[b]{2}{*}{ Model } & \multicolumn{3}{|c|}{$\begin{array}{l}\text { No-to-moderate drinkers } \\
\qquad(n=411)\end{array}$} & \multirow[b]{2}{*}{$\beta$} & \multirow{2}{*}{$\begin{array}{l}\text { Heavy drinkers } \\
\qquad \begin{array}{c}(n=112) \\
95 \% \mathrm{Cl}\end{array}\end{array}$} & \multirow[b]{2}{*}{ p-value } \\
\hline & & $\beta$ & $95 \% \mathrm{Cl}$ & p-value & & & \\
\hline \multirow[t]{5}{*}{ eLF\%* } & 1 & 0.314 & $0.106 ; 0.522$ & 0.003 & 0.615 & $0.203 ; 1.027$ & 0.004 \\
\hline & 2 & 0.223 & $0.036 ; 0.409$ & 0.019 & 0.632 & $0.259 ; 1.004$ & 0.001 \\
\hline & $2 a(+W C)$ & 0.071 & $-0.085 ; 0.228$ & 0.371 & 0.336 & $-0.002 ; 0.674$ & 0.051 \\
\hline & $2 b(+C 3)$ & -0.030 & $-0.195 ; 0.136$ & 0.725 & 0.288 & $-0.050 ; 0.626$ & 0.094 \\
\hline & 3 & -0.068 & $-0.216 ; 0.079$ & 0.362 & 0.150 & $-0.164 ; 0.465$ & 0.345 \\
\hline \multirow[t]{5}{*}{ LE score } & 1 & 0.136 & $-0.108 ; 0.380$ & 0.273 & 0.928 & $0.432 ; 1.423$ & $<0.001$ \\
\hline & 2 & 0.042 & $-0.198 ; 0.281$ & 0.732 & 0.917 & $0.443 ; 1.392$ & $<0.001$ \\
\hline & $2 a(+W C)$ & -0.038 & $-0.271 ; 0.196$ & 0.751 & 0.775 & $0.283 ; 1.266$ & 0.002 \\
\hline & $2 b(+C 3)$ & -0.153 & $-0.385 ; 0.080$ & 0.198 & 0.652 & $0.176 ; 1.128$ & 0.008 \\
\hline & 3 & -0.171 & $-0.401 ; 0.060$ & 0.146 & 0.605 & $0.119 ; 1.092$ & 0.015 \\
\hline \multirow[t]{5}{*}{ AST } & 1 & 0.036 & $-0.058 ; 0.130$ & 0.453 & 0.304 & $0.102 ; 0.507$ & 0.004 \\
\hline & 2 & 0.017 & $-0.077 ; 0.111$ & 0.719 & 0.303 & $0.101 ; 0.504$ & 0.004 \\
\hline & $2 a(+W C)$ & -0.004 & $-0.098 ; 0.089$ & 0.926 & 0.275 & $0.064 ; 0.487$ & 0.011 \\
\hline & $2 b(+C 3)$ & -0.050 & $-0.142 ; 0.043$ & 0.291 & 0.219 & $0.012 ; 0.427$ & 0.038 \\
\hline & 3 & -0.054 & $-0.146 ; 0.039$ & 0.255 & 0.218 & $0.005 ; 0.430$ & 0.045 \\
\hline \multirow[t]{5}{*}{ ALT } & 1 & 0.035 & $-0.082 ; 0.152$ & 0.559 & 0.441 & $0.218 ; 0.665$ & $<0.001$ \\
\hline & 2 & -0.013 & $-0.126 ; 0.101$ & 0.829 & 0.421 & $0.199 ; 0.642$ & $<0.001$ \\
\hline & $2 a(+W C)$ & -0.058 & $-0.167 ; 0.052$ & 0.301 & 0.353 & $0.124 ; 0.583$ & 0.003 \\
\hline & $2 b(+C 3)$ & -0.109 & $-0.219 ; 0.001$ & 0.053 & 0.322 & $0.095 ; 0.549$ & 0.006 \\
\hline & 3 & -0.120 & $-0.228 ;-0.012$ & 0.029 & 0.294 & $0.063 ; 0.526$ & 0.013 \\
\hline \multirow[t]{5}{*}{ GGT } & 1 & 0.140 & $-0.052 ; 0.331$ & 0.153 & 0.487 & $0.114 ; 0.861$ & 0.011 \\
\hline & 2 & 0.069 & $-0.123 ; 0.261$ & 0.482 & 0.504 & $0.160 ; 0.848$ & 0.005 \\
\hline & $2 a(+W C)$ & 0.030 & $-0.162 ; 0.222$ & 0.759 & 0.388 & $0.033 ; 0.743$ & 0.032 \\
\hline & $2 b(+C 3)$ & -0.021 & $-0.215 ; 0.174$ & 0.835 & 0.311 & $-0.034 ; 0.656$ & 0.077 \\
\hline & 3 & -0.030 & $-0.224 ; 0.164$ & 0.764 & 0.268 & $-0.083 ; 0.620$ & 0.133 \\
\hline
\end{tabular}

Bs are unstandardized regression coefficients and represent the change in (In-transformed) liver fat percentage, LE score and individual liver enzymes per 1 unit increase in In-transformed C3a. Model 1: adjusted for age and sex; Model 2: model 1 + adjusted for glucose metabolism status, cardiovascular disease, estimated glomerular filtration rate, smoking status, alcohol use, and use of medication; Model 3: model 2 + adjusted for waist circumference and $\mathrm{C} 3.95 \% \mathrm{Cl}$ : $95 \%$ confidence interval; eLF\%: estimated liver fat percentage; LE score: liver enzyme score; AST: aspartate amino transferase; ALT: alanine aminotransferase; GGT: gamma-glutamyl transferase; WC: waist circumference. * For eLF\%, model 2 did not include glucose metabolism status, since this variable was also used in the liver fat equation.

\section{Association of C3a with liver enzymes}

Although not related to $\mathrm{C} 3 \mathrm{a}$ in the overall population, the liver enzyme score did increase across tertiles of $\mathrm{C} 3 \mathrm{a}$ in heavy alcohol consumers (Figure 4.4.1). Similar increases across C3a tertiles were observed for all liver enzymes individually (data not shown). C3a was associated with the composite liver enzyme score in subjects with heavy alcohol consumption ( $\beta=0.917 ; 95 \% \mathrm{Cl} 0.443-1.392)$, but not in subjects with noto-moderate alcohol consumption $(\beta=0.042 ; 95 \% \mathrm{Cl}-0.198-0.281 ;$ P-interaction=0.001) (Table 4.4.2; model 2). In heavy alcohol consumers, this association was somewhat 
attenuated after adjustment for waist circumference $(\beta=0.775 ; 95 \% \mathrm{Cl} 0.283-1.266)$ or C3 $(\beta=0.652 ; 95 \% \mathrm{Cl} 0.176-1.128)$, but remained statistically significant. Moreover, C3a was still independently associated with the liver enzyme score after adjustment for both waist circumference and C3 $(\beta=0.605 ; 95 \% \mathrm{Cl} 0.119-1.092)$. Similar results were also observed for all liver enzymes individually (Table 4.4.2).

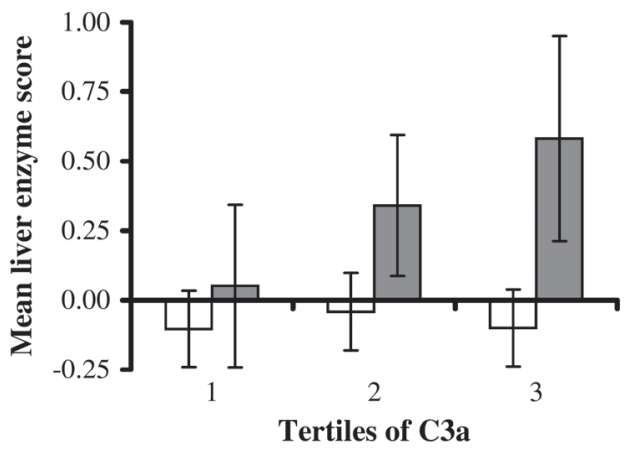

Figure 4.4.1 Bar chart of liver enzyme score according to tertiles of C3a stratified by alcohol use. White bars represent no-to-moderate drinkers $(n=411)$, black bars represent heavy drinkers $(n=112)$ who were defined using sex-specific cut-offs of $>30 \mathrm{~g} / \mathrm{d}$ for men and $>20 \mathrm{~g} / \mathrm{d}$ for women. $95 \%$ confidence intervals of group means are shown by the whiskers.

Next, we investigated whether the association between C3a and the liver enzyme score as observed in heavy alcohol consumers was mediated by TNF- $\alpha$ or insulin resistance. The association between $\mathrm{C} 3 \mathrm{a}$ and the liver enzyme score $(\beta=0.775 ; 95 \% \mathrm{Cl}$ 0.283-1.266; model 2a) was attenuated to $\beta=0.568(95 \% \mathrm{Cl} 0.076-1.061)$ after adjustment for TNF- $\alpha$, and to $\beta=0.650(95 \% \mathrm{Cl} 0.204-1.095)$ after adjustment for HOMA2-IR. The magnitude of mediation by TNF- $\alpha$ was $\beta=0.206(95 \% \mathrm{Cl} 0.036-0.527)$, explaining $27 \%$ of the overall association between $\mathrm{C} 3 \mathrm{a}$ and the liver enzyme score. Mediation by HOMA2-IR was less and not statistically significant (16\%; $\beta=0.125$; $95 \% \mathrm{Cl}-0.108$ to 0.445$)$.

In the present study, plasma C3a levels were not associated with liver enzymes in subjects with no-to-moderate alcohol consumption. However, activation of the complement system has been observed in liver biopsies of morbidly obese humans (mean BMI $48 \mathrm{~kg} / \mathrm{m}^{2}$ ) with non-alcoholic steatohepatitis (18). For this reason, we also investigated the associations of plasma C3a with liver fat content and the liver enzyme score in a subgroup of the most obese subjects (BMl>35 kg/m2; n=32) with no-to-moderate alcohol consumption. In these severely obese subjects, C3a showed a non-significant trend with estimated liver fat percentage (age- and sex-adjusted $\beta=0.300 ; 95 \% \mathrm{Cl}-0.687-1.288$ ). More importantly, the strength of the association of C3a 
with the liver enzyme score (age- and sex-adjusted $\beta=0.863 ; 95 \% \mathrm{Cl}-0.423-2.148$ ) was similar to the association observed in the heavy alcohol consumers $(\beta=0.928 ; 95 \% \mathrm{Cl}$ 0.432-1.423; Table 4.4.2: model 1).

\section{Additional analyses}

The cut-off value for alcohol consumption to distinguish NAFLD from AFLD is subject to discussion, and some propose a more stringent cut-off value of $20 \mathrm{~g} / \mathrm{d}$ for both sexes (28). A stratified analysis in subjects with alcohol consumption $<20 \mathrm{~g} / \mathrm{d}(\mathrm{n}=368)$ and $\geq 20 \mathrm{~g} / \mathrm{d}$ ( $\mathrm{n}=155$ ) respectively yielded results comparable to the main analysis (data not shown). In addition, plasma C3a concentrations might be elevated in the presence of infectious or inflammatory diseases at the time of blood sampling. However, associations between $\mathrm{C} 3 \mathrm{a}$ and liver outcomes did not materially change in an additional analysis excluding subjects with serum hs-CRP >10 mg/l ( $\mathrm{n}=40$; data not shown). In addition, similar associations were observed after exclusion of subjects with selfreported (history of) renal, intestinal, gastric, and bile duct disease $(n=54$, data not shown).

\section{Discussion}

The present study has three main findings. First, we showed that plasma C3a levels were associated with (estimated) liver fat percentage, and that these associations appeared to be more pronounced in heavy than no-to-moderate alcohol consumers. Second, in subjects with heavy alcohol consumption, C3a levels were independently associated with hepatocellular damage as defined by a composite score of AST, ALT, and GGT. Third, we showed that a substantial part ( 30\%) of the associations of C3a with either liver fat or liver enzymes in heavy alcohol consumers could be explained by related differences in TNF- $\alpha$. These findings suggest that in human subjects, complement activation may - partly via TNF- $\alpha$ - contribute to steatosis and hepatocellular injury in the metabolically compromised liver.

Several animal studies have found that complement knockout mice have decreased diet- or alcohol-induced liver steatosis and liver triglycerides $(10,11,15,17)$, but no human data are available on an association between complement activation (or C3a levels) and hepatic triglyceride content or steatosis. Also, complement activation in AFLD has not been studied previously in humans, to our knowledge. In this epidemiological study, we used plasma C3a levels as read-out of complement activation, and examined its association with two aspects of liver disease: liver steatosis and hepatocellular damage.

The present study did observe an association of C3a with liver fat content in humans in both no-to-moderate ("NAFLD") and heavy alcohol consumers ("AFLD"), but these 
associations were to a large extent attenuated after adjustment for abdominal adiposity (waist circumference) and/or C3. We included these adjustments to account for any potential (residual) confounding effects. However, abdominal adiposity (probably via the production of C3) may also be an important determinant of C3a levels $(21,22)$, and also of liver fat content $(30)$, and thus waist and/or C3 may be ascending proxies in the causal pathway between C3a and liver fat. Adjustments for ascending proxies are deemed unnecessary, and can attenuate estimates due to differences in bias and variance between C3a and waist circumference (31). In addition, waist circumference, is also used in the definition of the metabolic syndrome, a variable that is included in the liver fat equation (27). Given these considerations, and in the context of observational research, it is not possible to definitely disentangle whether waist (or C3) was a simple confounder or an ascending proxy, and our estimates (regression coefficients) of the associations between C3a and liver fat might have been underestimated due to some 'unnecessary' or overadjustment. Nevertheless, an independent association between $\mathrm{C} 3 \mathrm{a}$ and liver fat content was still observed in the heavy alcohol consumers, in agreement with animal experiments of alcoholic steatosis $(10,15,17)$. In addition, plasma TNF- $\alpha$ explained a substantial part of this association in the mediation analysis. Since (systemic) inflammation is also associated with (systemic) insulin resistance (32), C3a may contribute to hepatic steatosis via promotion of inflammation and insulin resistance, in agreement with the positive association between C3a and HOMA2-IR observed in our study (Table 4.4.1). Taken together, these results suggest that C3a may be involved in the pathogenesis of hepatic steatosis, at least in AFLD.

The independent associations between C3a levels and liver enzymes in the heavy alcohol consumers we report here support previous results from animal studies, which suggest that complement activation is involved in alcohol-induced liver injury $(10,14-17)$. The fact that we observed an association of C3a with liver enzymes only in heavy drinkers may suggest that complement-mediated hepatocellular damage is only present when the liver is vulnerable to injury. This agrees with the overall hypothesis that AFLD consists of a first hit that increases exposure of hepatocytes to TNF- $\alpha$, and a second hit that interferes with regular protective mechanisms against TNF- $\alpha$-induced cell death (5). Several mechanisms for this second hit have been proposed, most of which involve mitochondrial abnormalities, decreased antioxidative capacity and increased oxidative stress $(4,5)$. When combining our findings with the animal studies above, complement activation may be responsible for TNF- $\alpha$-mediated hepatocellular injury in vulnerable hepatocytes in the face of alcohol exposure. In agreement with this hypothesis, C3a-related variations in TNF- $\alpha$ were associated with the liver enzyme score in our mediation analysis.

Figure 4.4.2 summarises the proposed mechanisms via which C3a (and complement activation in general) may contribute to steatosis and hepatocellular injury in the metabolically compromised liver. Complement activation, e.g. by alcohol or LPS $(9,10)$, 
leads to formation of $\mathrm{C} 3 \mathrm{a}$ and $\mathrm{C} 3 \mathrm{~b}$ (6). C3a acts on the C3aR, leading to enhanced production and release of inflammatory cytokines, including TNF- $\alpha(12,15)$. In the liver, TNF- $\alpha$ derived from Kupffer cells may contribute to the development of hepatic steatosis, and is a key player in hepatic inflammation and apoptosis and necrosis of hepatocytes $(4,5)$. In addition, systemic (C3a-dependent) inflammation may contribute to peripheral insulin resistance (11), also leading to hepatic steatosis. Finally, complement activation / deposition in the liver may result in formation of the membrane attack complex (MAC) from distal complement factors C5-C9, which may also induce hepatic injury. Indeed, C8 deposition was strongly increased in the livers of alcohol-fed rats compared with control rats (14).

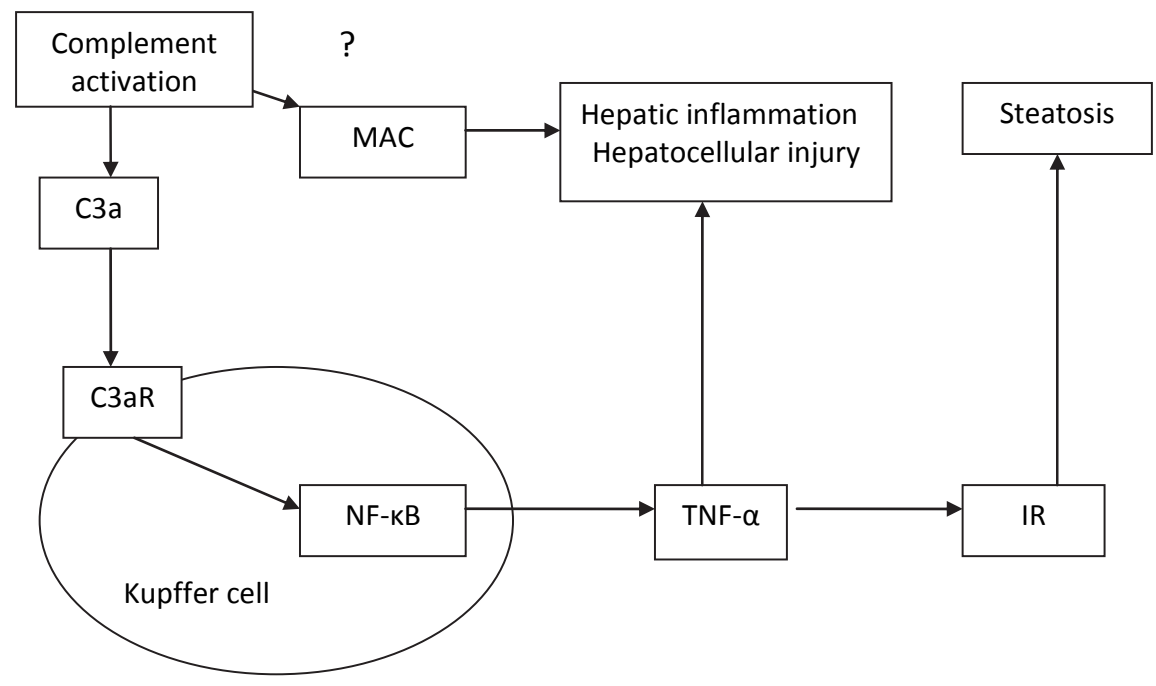

Figure 4.4.2 Proposed mechanism of complement system involvement in metabolic liver diseases: Complement activation may contribute to hepatic steatosis and hepatocellular injury through the interaction of the anaphylotoxin C3a with the C3a receptor (C3aR) on liver Kupffer cells. This activates nuclear factor-KB (NF-KB) leading to enhanced production and release of inflammatory cytokines, of which tumour necoris factor- $\alpha$ (TNF- $\alpha$ ) is the most important in liver diseases. TNF- $\alpha$ is involved in hepatic inflammation, apoptosis and necrosis of hepatocytes, but may also contribute to the development of hepatic steatosis, either direct or indirect through induction of insulin resistance (IR). Finally, complement activation may result in formation of the membrane attack complex (MAC) from distal complement factors $\mathrm{C5}-\mathrm{C}$, which may also induce hepatic injury.

Complement activation and deposition in the liver has also been observed in morbidly obese patients with NASH $(18,19)$. In an additional analysis in the most severely obese subjects with no-to-moderate alcohol consumption, we also observed a non-significant trend between C3a levels and liver enzymes. These results suggest that 
C3a may also play a role in non-alcoholic steatohepatitis and hepatocellular injury; stages that occur mainly in very obese subjects (30). In agreement, AST and ALT levels were lower in C3aR knockout mice on a high fat diet compared with wild type mice (11).

The present study is the first, to our knowledge, to investigate the association of plasma C3a levels with markers of liver disease in a relatively large cohort, with adjustment for several potential confounders. Still, some limitations need to be mentioned. We used an equation to estimate liver fat content, but did not measure liver fat content directly with sensitive imaging techniques, such as ultrasound or ${ }^{1} \mathrm{H}$-MRS. We did not perform liver biopsies, and hence were unable to determine subjects' degree of steatosis, steatohepatitis, or fibrosis on histological grounds, because of ethical concerns, since such procedure cannot be performed in the absence of a clinical indication. However, the use of a combined score of liver enzymes in a group of subjects with a wide range of alcohol consumption did provide an efficient way to analyze our data. Furthermore, and in support of its validity, the associations of C3a with the liver enzyme score were confirmed by associations of C3a with each of individual liver enzymes as well. Still, because the liver score is derived from the mean of Z-scores of each liver enzyme considered, and these Z-scores depend on the observed distribution of these variables in the population under study, our findings may not be directly comparable to other study populations due to different distributions. This does not impair its use for etiological research questions as in the present paper, however. The association with the liver enzyme score allows us to conclude that C3a is associated with liver disease, as liver enzymes are a sensitive marker for hepatocellular injury, are associated with mortality (33), and are higher in patients with AFLD or NAFLD than in subjects without liver disease (1). In addition, the cross-sectional design does not allow us to draw definite conclusions in terms of causality. In a reverse causation scenario, one could argue that in the face of chronic (alcoholic) hepatocyte damage and apoptosis, the complement system becomes activated and plasma C3a levels rise. However, the results from several complement knockout animal studies make this reverse causation scenario less likely (10,14-17). Finally, our study was conducted in middle-aged and older, Caucasian subjects who were selected on the basis of an increased risk for metabolic and cardiovascular disease, and extrapolation to other study populations (e.g. lean alcoholics) or other ethnicities should be done with caution.

In conclusion, we have provided evidence that C3a, a marker of complement activation, is associated with liver steatosis and hepatocellular injury in humans, at least in individuals who consume considerable amounts of alcohol daily, and presumably in severely obese persons as well. Moreover, increased production of TNF- $\alpha$ is a likely mechanism through which C3a may lead to hepatic steatosis and hepatocellular injury. Given the novelty of our findings, future (clinical) research, preferably using more direct measurements of liver fat and liver inflammation, is warranted. In addition, future 
studies should be directed at the study of the contribution of specific aspects of complement activation to both AFLD and NAFLD in humans to increase our understanding of the occurrence and progression of these diseases. 


\section{References}

1. Bedogni G, Miglioli L, Masutti F, Tiribelli C, Marchesini G, Bellentani S: Prevalence of and risk factors for nonalcoholic fatty liver disease: the Dionysos nutrition and liver study. Hepatology 2005;42:44-52

2. Wlazlo N, Beijers HJ, Schoon EJ, Sauerwein HP, Stehouwer CD, Bravenboer B: High prevalence of diabetes mellitus in patients with liver cirrhosis. Diabet Med 2010;27:1308-1311

3. Matteoni CA, Younossi ZM, Gramlich T, Boparai N, Liu YC, McCullough AJ: Nonalcoholic fatty liver disease: a spectrum of clinical and pathological severity. Gastroenterology 1999;116:1413-1419

4. Lucey MR, Mathurin P, Morgan TR: Alcoholic hepatitis. N Engl J Med 2009;360:2758-2769

5. Tilg H, Diehl AM: Cytokines in alcoholic and nonalcoholic steatohepatitis. N Engl J Med 2000;343: 1467-1476

6. Walport MJ: Complement. First of two parts. N Engl J Med 2001;344:1058-1066

7. Fischer WH, Jagels MA, Hugli TE: Regulation of IL-6 synthesis in human peripheral blood mononuclear cells by C3a and C3a(desArg). J Immunol 1999;162:453-459

8. Urbaschek R, McCuskey RS, Rudi V, Becker KP, Stickel F, Urbaschek B, Seitz HK: Endotoxin, endotoxinneutralizing-capacity, SCD14, sICAM-1, and cytokines in patients with various degrees of alcoholic liver disease. Alcohol Clin Exp Res 2001;25:261-268

9. Bykov I, Junnikkala S, Pekna M, Lindros KO, Meri S: Effect of chronic ethanol consumption on the expression of complement components and acute-phase proteins in liver. Clin Immunol 2007;124: 213-220

10. Pritchard MT, McMullen MR, Stavitsky AB, Cohen JI, Lin F, Medof ME, Nagy LE: Differential contributions of $\mathrm{C} 3, \mathrm{C} 5$, and decay-accelerating factor to ethanol-induced fatty liver in mice. Gastroenterology 2007;132:1117-1126

11. Mamane Y, Chung Chan C, Lavallee G, Morin N, Xu L, Huang J, Gordon R, Thomas W, Lamb J, Schadt EE, Kennedy BP, Mancini JA: The C3a anaphylatoxin receptor is a key mediator of insulin resistance and functions by modulating adipose tissue macrophage infiltration and activation. Diabetes 2009;58: 2006-2017

12. Strey CW, Markiewski M, Mastellos D, Tudoran R, Spruce LA, Greenbaum LE, Lambris JD: The proinflammatory mediators C3a and C5a are essential for liver regeneration. J Exp Med 2003;198: 913-923

13. Puschel GP, Hespeling $U$, Oppermann $M$, Dieter $P$ : Increase in prostanoid formation in rat liver macrophages (Kupffer cells) by human anaphylatoxin C3a. Hepatology 1993;18:1516-1521

14. Jarvelainen HA, Vakeva A, Lindros KO, Meri S: Activation of complement components and reduced regulator expression in alcohol-induced liver injury in the rat. Clin Immunol 2002;105:57-63

15. Roychowdhury S, McMullen MR, Pritchard MT, Hise AG, van Rooijen N, Medof ME, Stavitsky AB, Nagy LE: An early complement-dependent and TLR-4-independent phase in the pathogenesis of ethanolinduced liver injury in mice. Hepatology 2009;49:1326-1334

16. Cohen JI, Roychowdhury S, McMullen MR, Stavitsky AB, Nagy LE: Complement and alcoholic liver disease: role of $\mathrm{C} 1 \mathrm{q}$ in the pathogenesis of ethanol-induced liver injury in mice. Gastroenterology 2010;139:664-674, 674 e661

17. Bykov I, Junnikkala S, Pekna M, Lindros KO, Meri S: Complement C3 contributes to ethanol-induced liver steatosis in mice. Ann Med 2006;38:280-286

18. Rensen SS, Slaats Y, Driessen A, Peutz-Kootstra CJ, Nijhuis J, Steffensen R, Greve JW, Buurman WA: Activation of the complement system in human nonalcoholic fatty liver disease. Hepatology 2009;50:1809-1817

19. Yesilova Z, Ozata M, Oktenli C, Bagci S, Ozcan A, Sanisoglu SY, Uygun A, Yaman H, Karaeren N, Dagalp K: Increased acylation stimulating protein concentrations in nonalcoholic fatty liver disease are associated with insulin resistance. Am J Gastroenterol 2005;100:842-849

20. van Greevenbroek MM, Jacobs M, van der Kallen CJ, Vermeulen VM, Jansen EH, Schalkwijk CG, Ferreira I, Feskens EJ, Stehouwer CD: The cross-sectional association between insulin resistance and circulating complement C3 is partly explained by plasma alanine aminotransferase, independent of central obesity and general inflammation (the CODAM study). Eur J Clin Invest 2011;41:372-379 
21. Wlazlo N, van Greevenbroek MM, Ferreira I, Jansen EJ, Feskens EJ, van der Kallen CJ, Schalkwijk CG, Bravenboer B, Stehouwer CD: Low-grade inflammation and insulin resistance independently explain substantial parts of the association between body fat and serum C3: The CODAM study. Metabolism 2012;61:1787-1796

22. Weyer C, Tataranni PA, Pratley RE: Insulin action and insulinemia are closely related to the fasting complement C3, but not acylation stimulating protein concentration. Diabetes Care 2000;23:779-785

23. Jacobs M, van Greevenbroek MM, van der Kallen CJ, Ferreira I, Feskens EJ, Jansen EH, Schalkwijk CG, Stehouwer C: The association between the metabolic syndrome and alanine amino transferase is mediated by insulin resistance via related metabolic intermediates (the Cohort on Diabetes and Atherosclerosis Maastricht [CODAM] study). Metabolism 2011;60:969-975

24. Ocke MC, Bueno-de-Mesquita HB, Goddijn HE, Jansen A, Pols MA, van Staveren WA, Kromhout D: The Dutch EPIC food frequency questionnaire. I. Description of the questionnaire, and relative validity and reproducibility for food groups. Int J Epidemiol 1997;26 Suppl 1:S37-48

25. Levey AS, Bosch JP, Lewis JB, Greene T, Rogers N, Roth D: A more accurate method to estimate glomerular filtration rate from serum creatinine: a new prediction equation. Modification of Diet in Renal Disease Study Group. Ann Intern Med 1999;130:461-470

26. Alberti KG, Zimmet $P$, Shaw J: The metabolic syndrome--a new worldwide definition. Lancet 2005;366:1059-1062

27. Kotronen A, Peltonen M, Hakkarainen A, Sevastianova K, Bergholm R, Johansson LM, Lundbom N, Rissanen A, Ridderstrale M, Groop L, Orho-Melander M, Yki-Jarvinen H: Prediction of non-alcoholic fatty liver disease and liver fat using metabolic and genetic factors. Gastroenterology 2009;137:865-872

28. Neuschwander-Tetri BA, Caldwell SH: Nonalcoholic steatohepatitis: summary of an AASLD Single Topic Conference. Hepatology 2003;37:1202-1219

29. Preacher KJ, Hayes AF: Asymptotic and resampling strategies for assessing and comparing indirect effects in multiple mediator models. Behav Res Methods 2008;40:879-891

30. Angulo P: Nonalcoholic fatty liver disease. N Engl J Med 2002;346:1221-1231

31. Schisterman EF, Cole SR, Platt RW: Overadjustment bias and unnecessary adjustment in epidemiologic studies. Epidemiology 2009;20:488-495

32. Yudkin JS, Stehouwer CD, Emeis JJ, Coppack SW: C-reactive protein in healthy subjects: associations with obesity, insulin resistance, and endothelial dysfunction: a potential role for cytokines originating from adipose tissue? Arterioscler Thromb Vasc Biol 1999;19:972-978

33. Kim WR, Flamm SL, Di Bisceglie AM, Bodenheimer HC: Serum activity of alanine aminotransferase (ALT) as an indicator of health and disease. Hepatology 2008;47:1363-1370 


\section{Chapter 4.5}

Soluble C5b-9, but not C5a, is cross-sectionally associated with human fatty liver disease

Marleen MJ van Greevenbroek, Marjolein FJ Wilbrink, Elisabeth Hertle, Nick Wlazlo, Carla JH van der Kallen, Edith JM Feskens, Casper G Schalkwijk, Coen DA Stehouwer 


\section{Abstract}

The prevalence of fatty liver disease is increasing due to the current epidemic of obesity and related cardiometabolic diseases. However, the aetiological mechanisms that underlie fatty liver disease are incompletely understood. We previously showed that complement activation -as reflected by systemic C3a levels- was associated with human fatty liver disease. We now investigated whether activation of the terminal complement pathway -as represented by C5a and soluble (s)C5b-9- was also associated with fatty liver disease. For this, plasma C5a and sC5b-9 were measured in a Caucasian observational cohort ( $n=508,61 \%$ men; $59 \pm 7$ yrs). As markers of fatty liver disease we used liver fat content (eLF\%), estimated using a validated predictive equation, as well as liver enzymes [alanine aminotransferase (ALT), aspartate amino transferase (AST), and gamma-glutamyl transferase (GGT)], that were compiled into a standardized liver enzyme (LE) score. Multiple linear regression analyses were used to investigate cross-sectional associations between $\mathrm{C} 5 \mathrm{a}$ and $\mathrm{sC} 5 \mathrm{~b}-9$ and these fatty liver disease markers. Analyses were adjusted for age, sex, impaired glucose metabolism and type 2 diabetes mellitus, cardiovascular disease, smoking, alcohol consumption, kidney function, medication, physical activity, and waist circumference. In the fully adjusted regression models, standardized sC5b-9 was significantly associated with standardized eLF $(\beta=0.12(95 \% \mathrm{Cl}: 0.05 ; 0.18)$ and with the standardized LE-score ( $\beta=0.16$ ( $95 \% \mathrm{Cl}$ : 0.09;0.24). In contrast, $\mathrm{C} 5 \mathrm{a}$ was not significantly associated with either fatty liver disease marker. In conclusion, $\mathrm{SC} 5 \mathrm{~b}-9$ was associated with liver fat content and markers of hepatocellular injury, while $\mathrm{C} 5 \mathrm{a}$ appeared not to be associated with fatty liver disease. These data indicate that activation of the terminal complement pathway in the aetiology of human fatty liver disease deserves further investigation. 


\section{Introduction}

Alcoholic and non-alcoholic fatty liver disease (AFLD and NAFLD, respectively) represent the two most prevalent liver diseases in western society (1). Both consist of a spectrum ranging from steatosis to steatohepatitis and eventually liver fibrosis and cirrhosis. The prevalence of particularly NALFD has increased steeply over the past 1-2 decades, parallel to the rapidly developing obesity epidemic (2). There is a growing body of evidence that chronic (low-grade) inflammation and activation of the immune system contribute to both NAFLD and AFLD (3), but the details of the aetiology of fatty liver disease have remained largely elusive.

Inflammatory processes can be induced and regulated by the complement system, which is a complex protease network involved in immune surveillance and cell and tissue homeostasis (4). The complement system can be activated via the classical, the lectin or the alternative pathway. All three pathways converge in the activation of C3, resulting in the formation of the anaphylatoxin, C3a, and the opsonin, C3b. C3b is instrumental in the activation of $\mathrm{C} 5$ resulting in the generation of another important anaphylatoxin, $\mathrm{C} 5 \mathrm{a}$. The active component $\mathrm{C} 5 \mathrm{~b}$ then combines with $\mathrm{C} 6, \mathrm{C} 7, \mathrm{C} 8$ and $\mathrm{C} 9$ in a non-enzymatic way to form C5b-9 (also known as terminal complement complex; TCC or membrane attack complex; MAC). In sufficiently high concentrations, C5b-9 can cause cytolysis by formation of lytic pores on membranes (4). However, sublytic C5b-9 has also been reported to have various biological effects (5), including stimulation of endothelial cells, as reflected by increased expression of intercellular and vascular adhesion molecules and tissue factor (6), and enhancement of the effects of TNF $\alpha$ on endothelial cells (7).

Individuals prone to develop fatty liver disease, e.g. those who have type 2 diabetes mellitus (T2DM) or who consume considerable amounts of alcohol, have elevated circulating lipopolysaccharides (LPS) (8,9). Notably, LPS derived from microbes can activate the complement system (10). In addition, we have recently shown that increased activation of $\mathrm{C} 3$, as reflected by higher circulating concentrations of its activation product $\mathrm{C} 3 \mathrm{a}$, was associated with fatty liver disease in a human observational cohort (11). This is in line with studies in animal models that imply complement activation in the presence -and the development- of fatty liver disease (12-14). Moreover, morbidly-obese patients with non-alcoholic steatohepatitis showed hepatic deposition of both activated C3 and C5b-9 (15), suggesting involvement of full activation of the terminal pathway up to the formation of C5b-9 in human (N)AFLD.

Taking into account the above-mentioned data, we hypothesized that the terminal pathway of the complement system is activated in human fatty liver disease. Therefore, we investigated whether systemic concentrations of the complement activation products $\mathrm{C} 5 \mathrm{a}$ and $\mathrm{sC5}$-9 9 were associated with different aspects of fatty liver disease, i.e. hepatic steatosis and hepatocellular injury, in a middle-aged to older human population. 


\section{Materials and methods}

\section{Study population}

The Cohort on Atherosclerosis and Diabetes Maastricht (CODAM) study includes 574 individuals who were selected from a large population-based study on the basis of a moderately increased risk of T2DM and CVD (details are described elsewhere (16)). In short, inclusion criteria were Caucasian descent and age $>40$ years and, in addition, at least one of the following: $\mathrm{BMI}>25 \mathrm{~kg} / \mathrm{m}^{2}$; a positive family history of T2DM; a history of gestational diabetes and/or glucosuria; or use of antihypertensive medication. The present study reports on cross-sectional analyses in 508 individuals (61\% men). We derived at this study size by stepwise exclusion of individuals who had missing data on either of the main independent variables ( $C 5 a$ or sC5b-9, $n=16$ ), on the main outcomes (LE-score or eLF\%, $n=14$ ) and on any other covariate $(n=18)$, as well as those who were on insulin therapy $(n=12)$ or who had self-reported liver disease $(n=6)$. The study protocol was approved by the medical ethics committee of the Maastricht University and all CODAM participants gave written informed consent.

\section{Laboratory measurements}

Lipid-lowering medication was stopped 14 days before the measurements were obtained, other medication was stopped one day before the measurements. Blood samples were collected by venipuncture after an overnight fast. EDTA and citrate tubes were kept on ice until blood was centrifuged at $4^{\circ} \mathrm{C}$ (within 3 hours). Serum was allowed to clot at room temperature for 45 minutes. Aliquots were stored at $-80^{\circ} \mathrm{C}$ until use.

\section{Measurement of the complement factors (main independent variables)}

Aliquots were thawed rapidly at $37^{\circ} \mathrm{C}$ and subsequently transferred to ice. Complement C5a was measured in EDTA plasma by ELISA (MicroVue C5a EIA kit, Quidel, San Diego, USA). sC5b-9 was measured in citrate plasma by ELISA (MicroVue sC5b-9 EIA kit, Quidel). Inter-assay variation was $5.3 \%$ for $\mathrm{C} 5 \mathrm{a}$ and $11.8 \%$ for $\mathrm{sC} 5 \mathrm{~b}-9$.

\section{Markers of fatty liver disease (main dependent variables)}

Hepatic steatosis (reflected by estimated liver fat \%; eLF\%) was calculated using an earlier-published and validated predictive equation $(11,17)$. In this predictive equation aspartate aminotransferase (AST) concentrations, aminotransferase aminotransferase (AST/ALT) ratio, diabetes mellitus (yes/no), metabolic syndrome (yes/no) and fasting insulin concentrations are used. Glucose metabolism status was assessed by a standard $75 \mathrm{~g}$ OGTT and classified as normal glucose metabolism (NGM), impaired glucose metabolism (IGM, this group combined the individuals with impaired glucose tolerance 
and impaired fasting glucose) or T2DM according to the WHO criteria (18). Liver enzymes were measured using conventional clinical chemistry methods, metabolic syndrome was defined according to the International Diabetes Federation (19), and plasma insulin concentrations were determined as described before (20). The liver enzyme (LE) score was calculated as the mean of In-transformed, standardized liver enzymes (AST, ALT, gamma-glutamyl transferase GGT), thus creating a robust marker of hepatocellular injury with a relatively small measurement error (11).

\section{Measurements of other covariates}

All covariate measurements were obtained as previously described $(11,21)$. In short, waist circumference $(\mathrm{cm})$ was measured at the level midway between the lateral lower rib and the spina iliaca anterior superior, body mass index $\left(\mathrm{BMI}, \mathrm{kg} / \mathrm{m}^{2}\right)$ was calculated as weight divided by height squared. Smoking habits were assessed with a questionnaire about current and previous tobacco smoking (21). Physical activity was assessed with the use of a validated questionnaire (SQUASH (22)). Alcohol consumption was assessed by a validated food frequency questionnaire and divided into no-to-moderate ( $\leq 20 \mathrm{~g} / \mathrm{d}$ for women and $\leq 30 \mathrm{~g} / \mathrm{d}$ for men) and heavy (>20 g/d for women and $>30 \mathrm{~g} / \mathrm{d}$ for men) alcohol consumers $(11,23)$. Prior cardiovascular disease (CVD) was defined as self-reported cardiovascular disease, signs of ischaemic heart disease on electrocardiogram, amputation for non-traumatic reasons and/or AAlx $<0.9$ (24). Blood pressure was measured twice after 5 minutes of rest in supine position (25). In addition, total cholesterol, high-density lipoprotein (HDL) cholesterol, triglycerides, glucose and tumour necrosis factor (TNF)- $\alpha$ and C3a were measured in fasting EDTA, glucose was measured in NaF/KOx plasma and C-reactive protein (CRP) was measured in serum $(11,25)$. The estimated glomerular filtration rate (eGFR) was calculated from plasma creatinine levels using the MDRD formula $(11,26)$

\section{Statistical analysis}

Variables with a skewed distribution (ALT, AST, GGT, insulin, glucose, HOMA2-IR, C3a, TNF $\alpha$, eLF\% and triglycerides) were In transformed prior to further analyses. Characteristics of the study population were compared across tertiles of the markers of fatty liver disease (eLF\% and LE-score). Differences between groups were assessed using ANOVA (continuous variables), $\chi^{2}$ test (discrete variables) or Kruskal-Wallis test when transformations did not result in a normally distributed variable (i.e. smoking and alcohol consumption).

\section{Main analyses}

Multiple linear regression analyses were performed to evaluate the associations of C5a and sC5b-9 (independent variables) with eLF\% and the LE-score (dependent variables). These associations were adjusted for several potential confounders including age (yrs), 
sex, glucose tolerance status (T2DM, yes/no, impaired glucose metabolism (IGM), yes/no), cardiovascular disease (yes/no), smoking (packyears), alcohol consumption (no-to-moderate/heavy drinker), estimated glomerular filtration rate $\left(\mathrm{ml} / \mathrm{min} / 1.73 \mathrm{~m}^{2}\right)$, use of antihypertensive, lipid-lowering and/or glucose-lowering medication (each yes/no), total amount of physical activity (score) and waist circumference $(\mathrm{cm})$. Because glucose tolerance status (T2DM) was included in the calculation of liver fat \%, adjustment for glucose tolerance status was only performed in the analyses with LE-score as outcome. Prior to the regression analyses, C5a, sC5b-9, In-eLF\% and LEscore were each transformed into standardized values by -for each separate variablesubtracting the population mean from the individuals' observed value and dividing by the standard deviation (i.e. (individuals' observed value - population mean)/SD). This allows for direct comparisons of the strength of the associations of $\mathrm{C} 5 \mathrm{a}$ and sC5b-9 with eLF\% and LE-score, since effect sizes will be expressed per 1 SD change in the independent variable.

We previously observed a significant interaction between $\mathrm{C} 3 \mathrm{a}$ and alcohol consumption on fatty liver disease (11). In our current analyses we therefore included interaction terms ( $\mathrm{C} 5 \mathrm{a} \times$ alcohol consumption and $\mathrm{sC} 5 \mathrm{~b}-9 \mathrm{x}$ alcohol consumption) in the respective regression models. In that same study, TNF- $\alpha$ explained a significant proportion of the association between C3a and the LE-score (11), and it was shown by others that activation of the terminal complement may contribute to hepatic ischaemia-reperfusion damage via induction of TNF- $\alpha$ (27). Therefore, we additionally investigated whether the associations of $\mathrm{C} 5 \mathrm{a}$ and $\mathrm{sC} 5 \mathrm{~b}-9$ with fatty liver disease, if any, were explained (i.e. mediated) by TNF- $\alpha$.

\section{Additional analyses}

If the previously-reported association between C3a and fatty liver disease (11) was attributable to the anaphylatoxin properties of C3a and signalling via the C3a-receptor, C3a should be considered a potential confounder in the association between terminal pathway activation and fatty liver disease. It would then be prudent to adjust for C3a in the current analyses. On the other hand, systemic concentrations of C3a may very well mirror the overall activation state of the complement system, including terminal pathway activation. In that scenario, additional adjustment for C3a might induce overadjustment. For these reasons, we performed the multiple regression analyses without (main analyses) and with C3a (additional analyses) included as covariate. Moreover, since complement activation status may be affected by an immediate past history of infection of injury, we also repeated the analyses with exclusion of individuals with CRP concentrations higher than $10 \mathrm{mg} / \mathrm{l}$. Lastly, the distribution of C5a appeared to follow a bimodal distribution with a nadir at around $4 \mathrm{ng} / \mathrm{ml}$. Although this did not lead to violation of the assumptions underlying linear regression analyses, we did repeat the regression analyses including $\mathrm{C} 5 \mathrm{a}$ as a dichotomous variable $(<4.0$ versus $\geq 4.0 \mathrm{ng} / \mathrm{ml}$ ). 
P-values were considered significant when less than 0.05 for main analyses and when less than 0.1 for interaction analyses. IBM SPSS for Windows version 20 was used for all analyses.

\section{Results}

\section{Characteristics of study population}

Plasma concentrations of $\mathrm{C} 5 \mathrm{a}$ and $\mathrm{s} \mathrm{C} 5 \mathrm{~b}-9$ were positively and significantly associated (Spearman's $r$ was $0.15, p=0.001$ ). Table 4.5 .1 shows the characteristics of the study population according to tertiles of eLF\% and the LE-score. In short, individuals in the higher tertiles of both eLF\% and the LE-score were more often men, were more adipose, had higher tobacco consumption and more often had a disturbed glucose metabolism and/or dyslipidaemia. In contrast, individuals in the higher tertiles of eLF\% more often had prevalent CVD, had higher total cholesterol levels and more often used lipid-lowering medication, while this was not observed for tertiles of the LE-score. Furthermore, alcohol consumption did not differ across tertiles of estimated liver fat \%, while there was a gradual increase in alcohol intake from the lowest to the highest tertile of the LE-score. Table 4.5.1 also shows that C5a concentrations did not differ among tertiles of either eLF\% or the LE-score, while sC5b-9 concentration was highest in the upper tertile of both.

\section{Associations of $\mathrm{C} 5 \mathrm{a}$ and sC5b-9 with liver enzyme score and estimated liver fat content}

Age- and sex-adjusted C5a was associated with eLF\%: $\beta=0.09(95 \% \mathrm{Cl} 0.01 ; 0.18)$, but this association did not remain significant after adjustment for potential confounders $(\beta=0.07(95 \% \mathrm{Cl}-0.01 ; 0.14))$ and decreased even further after additional adjustment for waist circumference: $\beta=0.01(95 \% \mathrm{Cl}-0.05 ; 0.076)$ (Table 4.5 .2$)$. The association between $\mathrm{C} 5 \mathrm{a}$ and LE-score did not reach significance in any of the regression models. Similar results were obtained for all liver enzymes individually (Supplementary Table S4.5.1).

In contrast, we observed a positive and significant association between sC5b-9 and estimated liver fat \%, which remained significant in the fully adjusted model including waist circumference: $\beta=0.12(95 \% \mathrm{Cl} 0.05 ; 0.18)$. Thus, per 1 SD (i.e. $34 \mathrm{ng} / \mathrm{ml}$ ) difference in plasma sC5b-9, there was a $0.12 \mathrm{SD}$ higher eLF\% (In transformed). Likewise, there was a positive and significant association between sC5b-9 and the LE-score, which remained significant in the fully adjusted model including waist circumference: $\beta=0.16(95 \% \mathrm{Cl} 0.08 ; 0.24)$. As such, per $1 \mathrm{SD}$ difference in plasma sC5b-9, there was a 0.16 SD higher LE-score. Similar results were obtained for all three liver enzymes individually (Supplementary Table S4.5.1). 


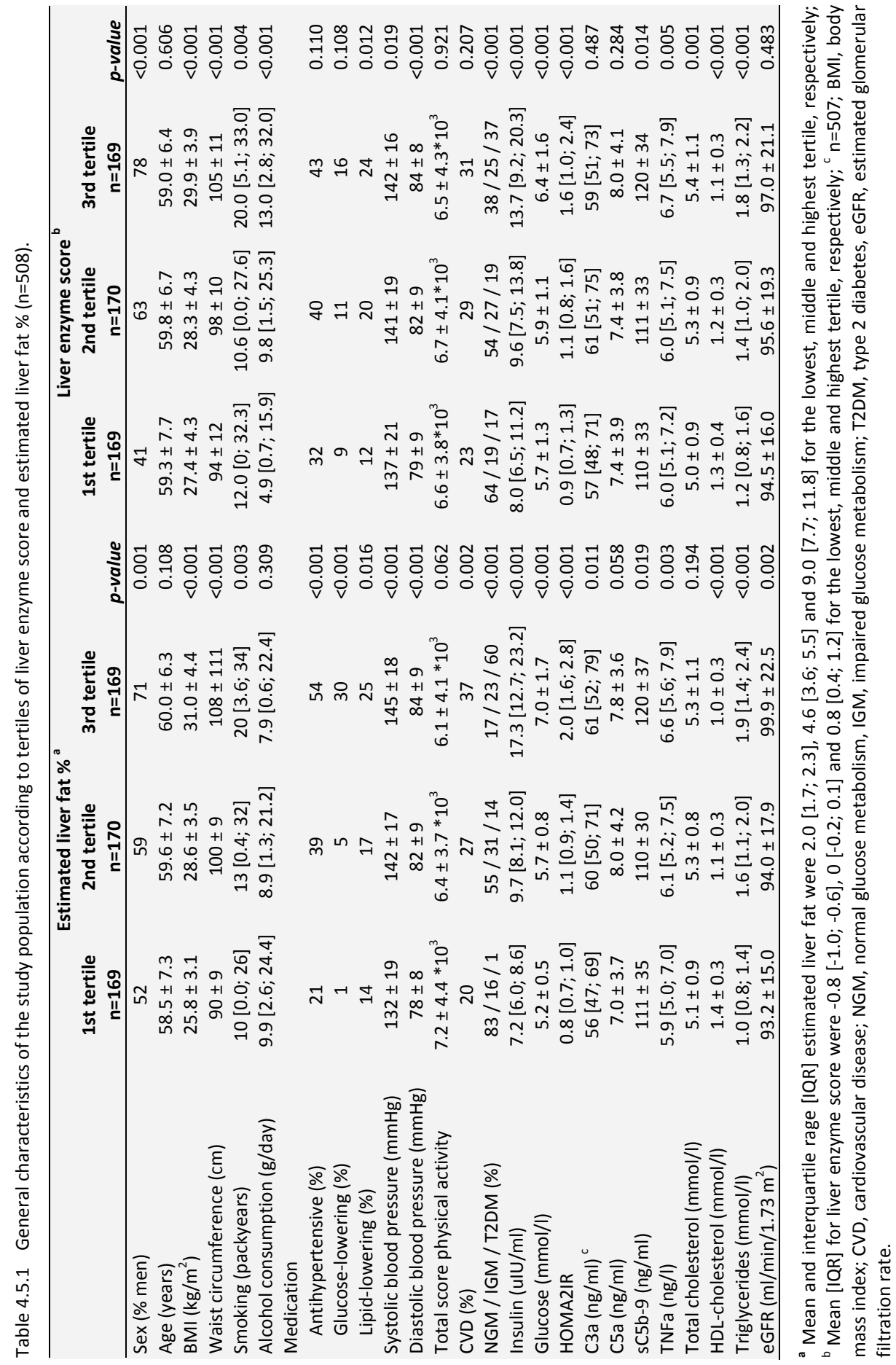


Table 4.5.2 Associations of with C5a and sC5b-9 (main predictors) with markers of fatty liver disease.

\begin{tabular}{llccclcrr}
\hline & & \multicolumn{3}{c}{ C5a (n=508) } & & & \multicolumn{3}{c}{ sC5b-9 (n=508) } \\
& & $\boldsymbol{\beta}$ & $\mathbf{9 5 \%}$ Cl & p-value & & $\boldsymbol{\beta}$ & 95\% Cl & p-value \\
\hline eLF\% & Model 1 & 0.074 & $-0.031 ; 0.162$ & 0.094 & Model 1 & 0.128 & $0.041 ; 0.215$ & 0.004 \\
& Model 2a & 0.092 & $0.006 ; 0.178$ & 0.036 & Model 2a & 0.144 & $0.058 ; 0.229$ & 0.001 \\
& Model 3 & 0.066 & $-0.011 ; 0.144$ & 0.093 & Model 3 & 0.129 & $0.052 ; 0.206$ & 0.001 \\
& Model 4 & 0.012 & $-0.052 ; 0.076$ & 0.710 & Model 4 & 0.116 & $0.052 ; 0.179$ & $<0.001$ \\
\hline LE-score & Model 1 & 0.046 & $-0.041 ; 0.133$ & 0.299 & Model 1 & 0.166 & $0.079 ; 0.258$ & $<0.001$ \\
& Model 2b & 0.055 & $-0.026 ; 0.136$ & 0.183 & Model 2b & 0.179 & $0.099 ; 0.258$ & $<0.001$ \\
& Model 3 & 0.056 & $-0.024 ; 0.136$ & 0.166 & Model 3 & 0.164 & $0.085 ; 0.244$ & $<0.001$ \\
& Model 4 & 0.031 & $-0.046 ; 0.108$ & 0.427 & Model 4 & 0.161 & $0.085 ; 0.237$ & $<0.001$ \\
\hline
\end{tabular}

Model $1=$ Crude association; Model 2a= Model $1+$ age, sex; Model $2 b=$ Model $1+$ age, sex, glucose metabolism status; Model 3= Model 2a or Model 2b + prevalent cardiovascular disease, smoking, kidney function, use of medication, physical activity; Model 4= Model $3+$ waist circumference. eLF\%, estimated liver fat \% (In transformed and standardized); LE-score, liver enzyme score (standardized).

Next, we evaluated whether the observed associations of C5a and sC5b-9 with eLF\% and LE-scores differed between no-to-moderate and heavy alcohol consumers. None of the interaction terms that were included in the fully adjusted regression models reached statistical significance (all $\mathrm{p}>0.15$ ). However, analyses for interaction are notoriously underpowered and therefore we re-analysed our study population stratified for alcohol consumption. These analyses showed that, although the effect sizes were substantially larger in the heavy alcohol consumers, the observed associations were consistently present in both the no-to-moderate and the heavy alcohol consumers (Tables 4.5.3A \& B).

Additionally, we evaluated to what extent TNF- $\alpha$ could explain, i.e. mediate, the observed associations of sC5b-9 with eLF\% and LE-score. For this, we added the plasma concentration of TNF- $\alpha$ as an additional covariate in the fully adjusted model presented in Table 4.5.2 (model 4). Addition of TNF- $\alpha$ had only a minimal effect on the strength of the associations of sC5b-9 with the markers of fatty liver disease under investigation. For eLF\% the regression coefficient changed from $\beta=0.116$ (Table 4.5.2, model 4) to $\beta=0.112(95 \% \mathrm{Cl} 0.05-0.18)$, i.e. a $3.4 \%$ decrease, and for LE-score it changed from $\beta=0.161$ (Table 4.5.2, model 4) to $\beta=0.156(95 \% \mathrm{Cl} 0.08 ; 0.23)$, i.e. a $3.1 \%$ decrease. Notably, in these full regression models TNF- $\alpha$ was, as expected, independently associated with eLF\% $(p=0.004)$ and with the LE-score $(p<0.001)$. Given the minimal effects of TNF- $\alpha$ on the strengths of the associations we did not perform further, more formal, mediation analyses. 
Table 4.5.3 Associations of with $\mathrm{C5a}$ and SC5b-9 (standardized) with estimated liver fat \% (A) and the LE-score (B), stratified for alcohol consumption and with additional adjustments.

\begin{tabular}{|c|c|c|c|c|c|c|c|}
\hline \multirow[t]{2}{*}{ A } & \multicolumn{6}{|c|}{ Estimated liver fat $\%$ as outcome } & \\
\hline & \multicolumn{3}{|c|}{ C5a } & & \multicolumn{3}{|c|}{ sC5b-9 } \\
\hline \multicolumn{7}{|c|}{ No-to-moderate alcohol consumption $(n=400)$} & \\
\hline & & $95 \% \mathrm{Cl}$ & p-value & & $\boldsymbol{\beta}$ & $95 \% \mathrm{Cl}$ & p-value \\
\hline Model 1 & 0.080 & $-0.019 ; 0.179$ & 0.111 & Model 1 & 0.108 & $0.009 ; 0.207$ & 0.032 \\
\hline Model 2a & 0.096 & $-0.002 ; 0.194$ & 0.054 & Model 2a & 0.126 & $0.028 ; 0.224$ & 0.011 \\
\hline Model 3 & 0.071 & $-0.017 ; 0.158$ & 0.112 & Model 3 & 0.121 & $0.033 ; 0.209$ & 0.007 \\
\hline Model 4 & 0.022 & $-0.051 ; 0.095$ & 0.551 & Model 4 & 0.100 & $0.027 ; 0.172$ & 0.007 \\
\hline Model 5 & 0.022 & $-0.051 ; 0.094$ & 0.559 & Model 5 & 0.103 & $0.031 ; 0.176$ & 0.005 \\
\hline \multicolumn{8}{|c|}{ Heavy alcohol consumption $(n=108)$} \\
\hline Model 1 & 0.057 & $-0.131 ; 0.245$ & 0.548 & Model 1 & 0.190 & $0.005 ; 0.376$ & 0.044 \\
\hline Model 2a & 0.044 & $-0.144 ; 0.231$ & 0.646 & Model 2a & 0.189 & $0.007 ; 0.371$ & 0.042 \\
\hline Model 3 & 0.021 & $-0.151 ; 0.194$ & 0.807 & Model 3 & 0.107 & $-0.071 ; 0.285$ & 0.237 \\
\hline Model 4 & -0.023 & $-0.166 ; 0.120$ & 0.751 & Model 4 & 0.158 & $0.013 ; 0.304$ & 0.033 \\
\hline Model 5 & -0.026 & $-0.168 ; 0.116$ & 0.716 & Model 5 & 0.144 & $-0.003 ; 0.291$ & 0.054 \\
\hline
\end{tabular}

Model 1= Crude association; Model 2a= Model $1+$ age, sex; Model 3= Model 2a + prevalent cardiovascular disease, smoking, kidney function, use of medication, physical activity; Model $4=$ Model $3+$ waist circumference; Model $5=$ Model $4+$ alcohol consumption $(\mathrm{g} / \mathrm{d})$. Analyses were additionally adjusted for alcohol intake in $\mathrm{g} / \mathrm{d}$ to ascertain that associations in any the stratified groups were not attributable to residual confounding.

\begin{tabular}{|c|c|c|c|c|c|c|c|}
\hline \multirow[t]{2}{*}{ B } & \multicolumn{6}{|c|}{ Liver enzyme score as outcome } & \multirow[b]{4}{*}{ P-value } \\
\hline & \multicolumn{3}{|c|}{ C5a } & & \multicolumn{2}{|r|}{ sC5b-9 } & \\
\hline \multicolumn{7}{|c|}{ No-to-moderate alcohol consumption $(n=400)$} & \\
\hline & $\beta$ & $95 \% \mathrm{Cl}$ & P-value & & $\beta$ & $95 \% \mathrm{Cl}$ & \\
\hline Model 1 & 0.061 & $-0.035 ; 0.157$ & 0.213 & Model 1 & 0.121 & $0.026 ; 0.216$ & 0.013 \\
\hline Model 2b & 0.063 & $-0.027 ; 0.153$ & 0.171 & Model 2b & 0.148 & $0.059 ; 0.237$ & 0.001 \\
\hline Model 3 & 0.062 & $-0.029 ; 0.152$ & 0.182 & Model 3 & 0.141 & $0.051 ; 0.231$ & 0.002 \\
\hline Model 4 & 0.042 & $-0.046 ; 0.129$ & 0.347 & Model 4 & 0.132 & $0.046 ; 0.219$ & 0.003 \\
\hline Model 5 & 0.042 & $-0.045 ; 0.130$ & 0.342 & Model 5 & 0.129 & $0.042 ; 0.216$ & 0.004 \\
\hline \multicolumn{8}{|c|}{ Heavy alcohol consumption $(n=108)$} \\
\hline Model 1 & -0.016 & $-0.178 ; 0.211$ & 0.867 & Model 1 & 0.269 & $0.081 ; 0.457$ & 0.006 \\
\hline Model 2b & -0.006 & $-0.186 ; 0.174$ & 0.948 & Model 2b & 0.226 & $0.048 ; 0.404$ & 0.013 \\
\hline Model 3 & -0.006 & $-0.193 ; 0.181$ & 0.952 & Model 3 & 0.247 & $0.057 ; 0.438$ & 0.011 \\
\hline Model 4 & -0.036 & $-0.216 ; 0.143$ & 0.689 & Model 4 & 0.282 & $0.102 ; 0.463$ & 0.003 \\
\hline Model 5 & -0.050 & $-0.225 ; 0.125$ & 0.572 & Model 5 & 0.257 & $0.078 ; 0.435$ & 0.005 \\
\hline
\end{tabular}

Model 1= Crude association; Model 2b= Model 1 + age, sex, glucose metabolism status; Model 3= Model 2b + prevalent cardiovascular disease, smoking, kidney function, use of medication, physical activity; Model 4= Model $3+$ waist circumference; Model $5=$ Model $4+$ alcohol consumption (g/d). Analyses were additionally adjusted for alcohol intake in $\mathrm{g} / \mathrm{d}$ to ascertain that associations in any the stratified groups were not attributable to residual confounding.

\section{Additional analyses}

When individuals with CRP $>10 \mathrm{mg} / \mathrm{l}(\mathrm{n}=37)$ were excluded, the associations of C5a with eLF\% and LE-score in the fully adjusted models remained weak and non-significant 
(data not shown). The association of SC5b-9 with eLF\% was somewhat attenuated but remained significant $(\beta=0.07$ (95\% Cl: $0.00 ; 0.14))$, while the association with LE-score was not materially altered $(\beta=0.15(95 \% \mathrm{Cl}$ : $0.06 ; 0.23))$.

When plasma concentration of C3a was added to the fully adjusted models the regression coefficient for the associations of $\mathrm{C} 5 \mathrm{a}$ did not materially change: $\beta=0.01$ (95\%Cl: $-0.05 ; 0.08)$ for eLF\%, and $\beta=0.03(95 \% \mathrm{Cl}:-0.04 ; 0.11)$ for LE-score. In addition, associations of sC5b-9 were not attenuated: $\beta=0.10(95 \% \mathrm{Cl}: 0.03-0.17)$ for eLF\%, and $\beta=0.15(95 \% \mathrm{Cl}: 0.08 ; 0.23)$ for LE-score (all $n=507$ since C3a was missing for 1 individual).

Lastly, when C5a was included as a dichotomous instead of a continuous variable in the fully adjusted regression models, the results for the associations with eLF\% and LEscore were again weak and not significant (both $\mathrm{p}>0.4$ ).

\section{Discussion}

The present study on the association between terminal pathway activation and fatty liver disease in a population of middle-aged to older individuals had four main findings. Firstly, we showed that systemic concentrations of the terminal pathway anaphylatoxin C5a were not associated with fatty liver disease, as represented by eLF\% and the LEscore. Secondly, we showed that $\mathrm{sC} 5 \mathrm{~b}-9$, as a marker of full terminal pathway activation, was strongly and independently associated with both eLF\% and LE-score. Thirdly, we showed that these latter associations were not mediated by systemic TNF- $\alpha$ levels. Fourthly, we showed that these associations were consistently present in both no-to-moderate and in heavy alcohol consumers, albeit somewhat stronger among those with the highest alcohol consumption. Taken together, these findings suggest that terminal complement activation up to the final step of C5b-9 assembly is important in human AFLD and NAFLD.

Human data on the potential role of complement activation in fatty liver disease are scarce but as far as they are available they do suggest that terminal pathway activation is involved. At first sight, our observation that $\mathrm{C} 5 \mathrm{a}$ and $\mathrm{sC5b}-9$, which are both generated upon activation of the terminal complement pathway, had distinct associations with markers of fatty liver disease was surprising. However, these factors are not generated in a one-to-one fashion during complement activation, and various (cellular) inhibitors are involved in controlling the assembly of the C5b-9 complex. In fact, in our population the correlation between systemic concentration of $\mathrm{C} 5 \mathrm{a}$ and sC5b-9 was, although statistically significant, not very strong (Spearman's $r$ was 0.15).

Our expectation of a positive association between $\mathrm{C} 5 \mathrm{a}$ and markers of fatty liver disease was based on both human and animal data. On the one hand, we previously showed that C3a, the other important complement anaphylatoxin, was associated with fatty liver disease, primarily in those with high alcohol intake (11). In addition, in 
patients with hepatitis B infection, C5a concentrations were associated with liver inflammation and liver fibrosis severity, and C5a was able to activate hepatic stellate cells in vitro (28). Moreover, C5 knockout mice were protected from hepatic injury and inflammation even though they were not protected from ethanol-induced hepatic steatosis (29). Despite these data, we have no indications that the different associations for $\mathrm{C} 5 \mathrm{a}$ and $\mathrm{sC} 5 \mathrm{~b}-9$ we observed can be explained by a difference in the quality of the measurements of the $\mathrm{C} 5 \mathrm{a}$ and $\mathrm{sC} 5 \mathrm{~b}-9$. For one thing, the inter-assay variation for the $\mathrm{C} 5 \mathrm{a}$ measurement (5.3\%) was even better than for $\mathrm{sC} 5 \mathrm{~b}-9$ (11.8\%). Also, associations one would theoretically expect for an anaphylatoxin such as C5a were essentially present in the CODAM population. For instance, C5a was positively and significantly associated with inflammatory markers such as CRP, IL-6, serum amyloid A, sICAM-1, ceruloplasmin and haptoglobin (Spearman's $r$ between 0.12 and 0.24 ; details not shown). Moreover, the strengths of these associations were highly comparable to those observed for sC5b-9 with the same inflammation markers. Given these observations, we see no clear reasons to assume that the C5a measurements that we obtained are not of sufficiently high quality for the analyses presented herein. Hence, our current observation that sC5b-9 was, but C5a was not, associated with eLF\% and LE-score appears to be a robust finding.

The positive associations of sC5b-9 with both fatty liver disease markers are in line with previous human studies. It has been shown that deposition of activated C3 is a very common finding in obese individuals with NAFLD (15). Moreover, 50\% of the liver biopsies that were positive for deposition of activated C3 also showed deposition of C5b-9 (15). This implies that full activation up to the final step of the terminal pathways is common in human NAFLD. Another study showed that sC5b-9 concentrations were higher in ascites and plasma of individuals with alcoholic liver cirrhosis (without infection) than in controls (9).

There are several possible mechanisms that may explain the observed differences between $\mathrm{C} 5 \mathrm{a}$ and $\mathrm{sC5b}-9$. First, several recent publications have implicated $\mathrm{C} 5 \mathrm{~b}-9$ $(30,31)$ and also C3a $(32)$ but not C5a $(30,32)$ in the activation of the NLR family, pyrin domain-containing 3 (NLRP3) inflammasome. Recent studies using different mouse models showed that inflammasome activation and IL-1 $\beta$ may be functionally involved in both alcoholic (33) and non-alcoholic (34) fatty liver disease. Higher inflammasome activation and IL-1 $\beta$ production have also been implicated in human fatty liver disease (35) and increased expression of NLRP3 inflammasome was reported in human steatohepatitis (36). Thus, these data in the literature strongly imply that full terminal pathway activation can contribute to fatty liver disease via activation of the inflammasome. This could explain our finding that the associations with the markers of fatty liver disease were stronger for sC5b-9 than for $\mathrm{C} 5 \mathrm{a}$. Moreover, inflammasome activation specifically results in production of activated IL-1 $\beta$ and IL-18, which may explain the lack of mediation by TNF- $\alpha$ in our current analyses. Secondly, the localized sequestration of potentially active sC5b-9 in the liver (and spleen) in comparison to the 
more generalized sequestration of $\mathrm{C} 5 \mathrm{a}$ into various highly vascularized tissues might, to some extent, also contribute to the observed difference in the association of $\mathrm{C5}$ a and sC5b-9 with markers of fatty liver disease $(37,38)$. Lastly, the lack of association for C5a might be explained by the fact that the assay we used detects not only $\mathrm{C} 5 \mathrm{a}$, but also its primary degradation product, C5a-desarg. C5a and C5a-desArg can each bind to both the $\mathrm{C} 5 \mathrm{a}$ receptor $(\mathrm{CD} 88 / \mathrm{C5aR})$ as well as to $\mathrm{C} 5 \mathrm{~L} 2$, although $\mathrm{C} 5 \mathrm{a}$ preferentially binds to the C5aR and C5a-desarg preferentially binds C5L2 (reviewed in (39)). Signalling via $\mathrm{C} 5 \mathrm{aR}$ is primarily pro-inflammatory but the physiological effects of C5L2 have not yet been fully determined and both proinflammatory and decoy functions have been proposed. When acting as decoy, binding of C5a and C5a-desarg to C5L2 might actually neutralize an effect of signalling via the $\mathrm{C} 5 \mathrm{aR}$ and, as such, blunt the effects of $\mathrm{C5}$ a on the liver (if any).

The main strength of this study is that we obtained high quality measurements of both $\mathrm{C} 5 \mathrm{a}$ and $\mathrm{sC5b}-9$ in a large human population. Furthermore, the extensive phenotyping that has been performed in the CODAM study allowed us to perform rigorous adjustments for potential confounders. However, our current study also has limitations. Firstly, we did not obtain direct measurements of hepatic fat accumulation and fatty liver disease, e.g. via ${ }^{1} \mathrm{H}-\mathrm{MRS}$ or a liver biopsy. Reasons for this include the high costs for ${ }^{1} \mathrm{H}-\mathrm{MRS}$, which makes this hard to implement in epidemiological cohorts, and the fact that CODAM participants did not have clinical signs of liver disease, which renders a liver biopsy unethical, given the associated health risks. As an alternative, we used two different measures of liver disease, i.e. eLF\% and circulating liver enzymes. The equation we used to estimate LF\% has been validated in prior studies and has proven to be a cost effective marker for fatty liver disease in epidemiologic research (17). The liver enzymes we used are a sensitive marker for hepatocellular injury and were shown to be higher in patients with (N)AFLD than in individuals without liver disease (40). Secondly, we used systemic concentrations of C5a and sC5b-9 as proxy measures of local terminal complement pathway activation. It has been shown that the half-life of $\mathrm{C} 5 \mathrm{a}$ is even shorter than for $\mathrm{C} 5 \mathrm{a}$-desarg, although for both it was less than 2 min (38) while sC5b-9 had a somewhat longer the half-life of $\pm 0.7 \mathrm{hrs}$ (37), which might contribute to the lack of association observed for C5a. However, in our study $\mathrm{C} 5 \mathrm{a}$ and $\mathrm{sC5b}-9$ were equally associated with inflammatory markers, and therefore this is unlikely to (fully) explain the observed differences between $\mathrm{C} 5 \mathrm{a}$ and sC5b-9 with respect to fatty liver disease. Still, the development of (N)AFLD may to some extent be a locally confined process and intrahepatic generation of $\mathrm{C} 5 \mathrm{a}$, with direct effect on e.g. Kupffer or hepatic stellate cells (41), may contribute to hepatic damage without being reflected in systemic concentrations of C5a / C5a-desarg. Third, the cross-sectional design of these evaluations prohibits conclusions on causality, and we need to take into account the possibility of reversed causation. However, sublytic C5b-9 was shown to induce inflammation and apoptosis (31), and also the results of several mouse models of interrupted complement activation speak against a reversed 
causation scenario $(12,13,29)$. Lastly, the CODAM study population is enriched with individuals with risk factors for cardiovascular disease and diabetes mellitus and extrapolation of the data to the general population should only be done with caution. This does, however, not impair its value for etiological research and our study population does represent a large group of individuals who are present in the population and in whom terminal complement activation might contribute to the development of fatty liver disease and its associated health threats.

In conclusion we have provided, for the first time, epidemiological data that point towards full activation of the terminal complement pathway, up to the formation of C5b-9 as a contributor to human fatty liver disease. Our current data do not support an important role for the anaphylatoxin $\mathrm{C} 5 \mathrm{a}$ in human fatty liver disease. These data clearly need confirmation and further investigation, on the one hand by evaluating, in longitudinal human studies, to what extent terminal pathway activation precedes the development of (N)AFLD, and on the other hand by evaluating the effects of manipulating terminal pathway activity (e.g. via C9 or CD59 depletion) in relation to development of high fat diet- or alcohol-induced fatty liver disease in genetically modified mouse models. These combined approaches will increase our understanding of the role of terminal complement activation in the aetiology of fatty liver disease and its complications, such as cardiometabolic diseases and hepatocellular cancer. 


\section{References}

1. Scaglioni F, Ciccia S, Marino M, Bedogni G, Bellentani S: ASH and NASH. Dig Dis 2011;29:202-210

2. Blachier M, Leleu H, Peck-Radosavljevic M, Valla DC, Roudot-Thoraval F: The burden of liver disease in Europe: a review of available epidemiological data. J Hepatol 2013;58:593-608

3. Kubes $P$, Mehal WZ: Sterile inflammation in the liver. Gastroenterology 2012;143:1158-1172

4. Ricklin D, Hajishengallis G, Yang K, Lambris JD: Complement: a key system for immune surveillance and homeostasis. Nat Immunol 2010;11:785-797

5. Tegla CA, Cudrici C, Patel S, Trippe R, 3rd, Rus V, Niculescu F, Rus H: Membrane attack by complement: the assembly and biology of terminal complement complexes. Immunol Res 2011;51:45-60

6. Tedesco F, Pausa M, Nardon E, Introna M, Mantovani A, Dobrina A: The cytolytically inactive terminal complement complex activates endothelial cells to express adhesion molecules and tissue factor procoagulant activity. J Exp Med 1997;185:1619-1627

7. Kilgore KS, Shen JP, Miller BF, Ward PA, Warren JS: Enhancement by the complement membrane attack complex of tumor necrosis factor-alpha-induced endothelial cell expression of E-selectin and ICAM-1. J Immunol 1995;155:1434-1441

8. de Kort S, Keszthelyi D, Masclee AA: Leaky gut and diabetes mellitus: what is the link? Obes Rev 2011;12:449-458

9. Bjerre $M$, Holland-Fischer $P$, Gronbaek $H$, Frystyk J, Hansen TK, Vilstrup H, Flyvbjerg A: Soluble membrane attack complex in ascites in patients with liver cirrhosis without infections. World J Hepatol 2010;2:221-225

10. Malik TH, Cortini A, Carassiti D, Boyle JJ, Haskard DO, Botto M: The alternative pathway is critical for pathogenic complement activation in endotoxin- and diet-induced atherosclerosis in low-density lipoprotein receptor-deficient mice. Circulation 2010;122:1948-1956

11. Wlazlo N, van Greevenbroek MM, Ferreira I, Jansen EH, Feskens EJ, van der Kallen CJ, Schalkwijk CG, Bravenboer B, Stehouwer CD: Activated complement factor 3 is associated with liver fat and liver enzymes: the CODAM study. Eur J Clin Invest 2013;43:679-688

12. Bykov I, Jauhiainen M, Olkkonen VM, Saarikoski ST, Ehnholm C, Junnikkala S, Vakeva A, Lindros KO, Meri S: Hepatic gene expression and lipid parameters in complement C3(-/-) mice that do not develop ethanol-induced steatosis. J Hepatol 2007;46:907-914

13. Roychowdhury S, McMullen MR, Pritchard MT, Hise AG, van Rooijen N, Medof ME, Stavitsky AB, Nagy LE: An early complement-dependent and TLR-4-independent phase in the pathogenesis of ethanolinduced liver injury in mice. Hepatology 2009;49:1326-1334

14. Mamane Y, Chung Chan C, Lavallee G, Morin N, Xu L, Huang J, Gordon R, Thomas W, Lamb J, Schadt EE, Kennedy BP, Mancini JA: The C3a anaphylatoxin receptor is a key mediator of insulin resistance and functions by modulating adipose tissue macrophage infiltration and activation. Diabetes 2009;58: 2006-2017

15. Rensen SS, Slaats Y, Driessen A, Peutz-Kootstra CJ, Nijhuis J, Steffensen R, Greve JW, Buurman WA: Activation of the complement system in human nonalcoholic fatty liver disease. Hepatology 2009;50:1809-1817

16. Kruijshoop M, Feskens EJ, Blaak EE, de Bruin TW: Validation of capillary glucose measurements to detect glucose intolerance or type 2 diabetes mellitus in the general population. Clin Chim Acta 2004;341:33-40

17. Kotronen A, Peltonen M, Hakkarainen A, Sevastianova K, Bergholm R, Johansson LM, Lundbom N, Rissanen A, Ridderstrale M, Groop L, Orho-Melander M, Yki-Jarvinen H: Prediction of non-alcoholic fatty liver disease and liver fat using metabolic and genetic factors. Gastroenterology 2009;137:865-872

18. Alberti KG, Zimmet PZ: Definition, diagnosis and classification of diabetes mellitus and its complications. Part 1: diagnosis and classification of diabetes mellitus provisional report of a WHO consultation. Diabet Med 1998;15:539-553

19. Alberti KG, Zimmet P, Shaw J: The metabolic syndrome--a new worldwide definition. Lancet 2005;366: 1059-1062 
20. van Greevenbroek MM, Jacobs M, van der Kallen CJ, Vermeulen VM, Jansen EH, Schalkwijk CG, Ferreira I, Feskens EJ, Stehouwer CD: The cross-sectional association between insulin resistance and circulating complement C3 is partly explained by plasma alanine aminotransferase, independent of central obesity and general inflammation (the CODAM study). Eur J Clin Invest 2011;41:372-379

21. van Greevenbroek MM, Jacobs $M$, van der Kallen $C J$, Blaak EE, Jansen EH, Schalkwijk CG, Feskens EJ, Stehouwer CD: Human plasma complement C3 is independently associated with coronary heart disease, but only in heavy smokers (the CODAM study). Int J Cardiol 2012;154:158-162

22. Wendel-Vos GC, Schuit AJ, Saris WH, Kromhout D: Reproducibility and relative validity of the short questionnaire to assess health-enhancing physical activity. J Clin Epidemiol 2003;56:1163-1169

23. Ocke MC, Bueno-de-Mesquita HB, Goddijn HE, Jansen A, Pols MA, van Staveren WA, Kromhout D: The Dutch EPIC food frequency questionnaire. I. Description of the questionnaire, and relative validity and reproducibility for food groups. Int J Epidemiol 1997;26 Suppl 1:S37-48

24. Wlazlo N, van Greevenbroek MM, Ferreira I, Jansen EJ, Feskens EJ, van der Kallen CJ, Schalkwijk CG, Bravenboer B, Stehouwer CD: Low-grade inflammation and insulin resistance independently explain substantial parts of the association between body fat and serum C3: The CODAM study. Metabolism 2012;61:1787-1796

25. Jacobs M, van Greevenbroek MM, van der Kallen CJ, Ferreira I, Blaak EE, Feskens EJ, Jansen EH, Schalkwijk CG, Stehouwer CD: Low-grade inflammation can partly explain the association between the metabolic syndrome and either coronary artery disease or severity of peripheral arterial disease: the CODAM study. Eur J Clin Invest 2009;39:437-444

26. Levey AS, Bosch JP, Lewis JB, Greene T, Rogers N, Roth D: A more accurate method to estimate glomerular filtration rate from serum creatinine: a new prediction equation. Modification of Diet in Renal Disease Study Group. Ann Intern Med 1999;130:461-470

27. Zhang J, Hu W, Xing W, You T, Xu J, Qin X, Peng Z: The protective role of CD59 and pathogenic role of complement in hepatic ischemia and reperfusion injury. Am J Pathol 2011;179:2876-2884

28. Xu R, Lin F, He J, Jin L, Zhang JY, Fu J, Liu H, Wang S, Zhang Z, Wang FS: Complement 5a stimulates hepatic stellate cells in vitro, and is increased in the plasma of patients with chronic hepatitis $B$. Immunology 2013;138:228-234

29. Pritchard MT, McMullen MR, Stavitsky AB, Cohen JI, Lin F, Medof ME, Nagy LE: Differential contributions of C3, C5, and decay-accelerating factor to ethanol-induced fatty liver in mice. Gastroenterology 2007;132:1117-1126

30. Laudisi F, Spreafico R, Evrard M, Hughes TR, Mandriani B, Kandasamy M, Morgan BP, Sivasankar B, Mortellaro A: Cutting Edge: The NLRP3 Inflammasome Links Complement-Mediated Inflammation and IL-1beta Release. J Immunol 2013;191:1006-1010

31. Triantafilou K, Hughes TR, Triantafilou M, Morgan BP: The complement membrane attack complex triggers intracellular Ca2+ fluxes leading to NLRP3 inflammasome activation. J Cell Sci 2013;126: 2903-2913

32. Asgari E, Le Friec G, Yamamoto H, Perucha E, Sacks SS, Kohl J, Cook HT, Kemper C: C3a modulates IL1beta secretion in human monocytes by regulating ATP efflux and subsequent NLRP3 inflammasome activation. Blood 2013; DOI 10.1182/blood-2013-05-502229

33. Petrasek J, Bala S, Csak T, Lippai D, Kodys K, Menashy V, Barrieau M, Min SY, Kurt-Jones EA, Szabo G: IL1 receptor antagonist ameliorates inflammasome-dependent alcoholic steatohepatitis in mice. J Clin Invest 2012;122:3476-3489

34. Miura K, Yang L, van Rooijen N, Brenner DA, Ohnishi H, Seki E: Toll-like receptor 2 and palmitic acid cooperatively contribute to the development of nonalcoholic steatohepatitis through inflammasome activation in mice. Hepatology 2013;57:577-589

35. Szabo G, Csak T: Inflammasomes in liver diseases. J Hepatol 2012;57:642-654

36. Csak T, Ganz M, Pespisa J, Kodys K, Dolganiuc A, Szabo G: Fatty acid and endotoxin activate inflammasomes in mouse hepatocytes that release danger signals to stimulate immune cells. Hepatology 2011;54:133-144

37. Greenstein JD, Peake PW, Charlesworth JA: The kinetics and distribution of C9 and SC5b-9 in vivo: effects of complement activation. Clin Exp Immunol 1995;100:40-46

38. Webster RO, Larsen GL, Henson PM: In vivo clearance and tissue distribution of C5a and C5a des arginine complement fragments in rabbits. J Clin Invest 1982;70:1177-1183 
39. Klos A, Tenner AJ, Johswich KO, Ager RR, Reis ES, Kohl J: The role of the anaphylatoxins in health and disease. Mol Immunol 2009;46:2753-2766

40. Bedogni G, Miglioli L, Masutti F, Tiribelli C, Marchesini G, Bellentani S: Prevalence of and risk factors for nonalcoholic fatty liver disease: the Dionysos nutrition and liver study. Hepatology 2005;42:44-52

41. Schieferdecker HL, Schlaf G, Jungermann K, Gotze O: Functions of anaphylatoxin C5a in rat liver: direct and indirect actions on nonparenchymal and parenchymal cells. Int Immunopharmacol 2001;1:469-481 


\section{Supplementary table}

Table S4.5.1 Associations of C5a and sC5b-9 (main predictors) with individuals liver enzymes.

\begin{tabular}{lcccccccr}
\hline & & \multicolumn{3}{c}{ C5a (n=508) } & & & \multicolumn{3}{c}{ sC5b-9 (n=508) } \\
& & $\boldsymbol{\beta}$ & $\mathbf{9 5 \%} \mathbf{~ C l}$ & P-value & & $\boldsymbol{\beta}$ & $\mathbf{9 5 \% ~ C l ~}$ & P-value \\
\hline ALT & Model 1 & 0.042 & $-0.045 ; 0.130$ & 0.952 & Model 1 & 0.145 & $0.059 ; 0.232$ & 0.001 \\
& Model 2 & 0.047 & $-0.034 ; 0.128$ & 0.253 & Model 2 & 0.157 & $0.078 ; 0.237$ & $<0.001$ \\
& Model 3 & 0.051 & $-0.030 ; 0.131$ & 0.219 & Model 3 & 0.149 & $0.069 ; 0.229$ & $<0.001$ \\
& Model 4 & 0.021 & $-0.055 ; 0.098$ & 0.582 & Model 4 & 0.146 & $0.070 ; 0.221$ & $<0.001$ \\
AST & Model 1 & 0.062 & $-0.025 ; 0.148$ & 0.163 & Model 1 & 0.187 & $0.102 ; 0.273$ & $<0.001$ \\
& Model 2 & 0.069 & $-0.017 ; 0.155$ & 0.114 & Model 2 & 0.197 & $0.113 ; 0.281$ & $<0.001$ \\
& Model 3 & 0.072 & $-0.013 ; 0.156$ & 0.095 & Model 3 & 0.184 & $0.100 ; 0.268$ & $<0.001$ \\
& Model 4 & 0.053 & $-0.030 ; 0.136$ & 0.212 & Model 4 & 0.181 & $0.099 ; 0.263$ & $<0.001$ \\
GGT & Model 1 & 0.015 & $-0.073 ; 0.102$ & 0.744 & Model 1 & 0.092 & $0.004 ; 0.179$ & 0.039 \\
& Model 2 & 0.045 & $-0.039 ; 0.129$ & 0.291 & Model 2 & 0.104 & $0.023 ; 0.184$ & 0.012 \\
& Model 3 & 0.035 & $-0.046 ; 0.116$ & 0.399 & Model 3 & 0.088 & $0.008 ; 0.169$ & 0.031 \\
& Model 4 & 0.013 & $-0.067 ; 0.093$ & 0.752 & Model 4 & 0.086 & $0.008 ; 0.165$ & 0.032 \\
\hline
\end{tabular}

Model 1= Crude association; Model 2= Model $1+$ age, sex, glucose metabolism status; Model 3= Model $2+$ prevalent cardiovascular disease, smoking, kidney function, use of medication, physical activity; Model 4= Model 3 + waist circumference. ALT: alanine aminotransferase alanine (In transformed and standardized); AST: aspartate aminotransferase (In transformed and standardized); GGT: gamma-glutamyl transferase (In transformed and standardized). 
Diabetes mellitus and cirrhosis

\section{Chapter 5

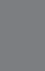

Cha (1) 


\section{Chapter}

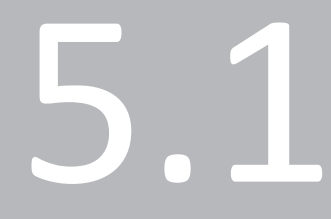

\section{The relation between diabetes mellitus and liver cirrhosis}

Nick Wlazlo, Hans P Sauerwein, Erik J Schoon, Coen DA Stehouwer, Bert Bravenboer Published in part in Dutch Ned Tijdschr Geneesk 2011;155:212-219; A2213 
Chapter 5.1 


\section{Introduction into chronic liver diseases and cirrhosis}

Liver cirrhosis is defined as the replacement of normal liver tissue by fibrous scarring tissue in response to chronic liver injury, which may have several causes, with resulting impairment of liver function and end-stage liver disease (1). Its reported prevalence of $0.15 \%$ in the USA is probably an underestimation, since compensated cirrhosis may exist for several years without clinical manifestations, leading to a high rate of undiagnosed cirrhosis among patients with viral hepatitis and non-alcoholic steatohepatitis (NASH). As such, a reasonable estimate of the prevalence of histological cirrhosis approximates $1 \%(1)$. Liver cirrhosis is still a disease with an unfavourable prognosis, as mortality rates have not improved during 1968-1999, and case-fatality rates 1 year after admission for cirrhosis are 33\% (2). Moreover, standardized mortality rates (per 100,000) for liver cirrhosis in the Netherlands have increased from 4.7 (women) and 7.8 (men) in 1957-1961 to 5.9 (women) and 9.7 (men) in 1997-2001 (3).

The aetiology of cirrhosis consists of several chronic liver diseases, including alcoholic liver disease (ALD), the spectrum of non-alcoholic fatty liver disease (NAFLD) and NASH, chronic viral hepatitis B or C, auto-immune liver diseases (auto-immune hepatitis, primary biliary cirrhosis, primary sclerosing cholangitis), metabolic diseases (e.g. hereditary haemochromatosis, Wilson's disease, alpha 1-antitrypsin deficiency), chronic heart failure, and medication. In case extensive evaluation for these chronic liver diseases yields no specific cause, cirrhosis is called 'cryptogenic'. Whereas hepatitis $B$ is the most common cause of cirrhosis in developing countries (4), alcohol and hepatitis $C$ are the prevailing causes in the Western world $(2,5)$, with NAFLD/NASH rising rapidly due to an increasing prevalence of obesity, the metabolic syndrome and type 2 diabetes mellitus (T2DM) $(6,7)$.

Chronic injury to the liver, regardless of the cause, may lead to necrosis and apoptosis of hepatocytes, and eventually to a wound-healing response, with hepatic stellate cells producing collagenous connective tissue (liver fibrosis) (8). In a healthy liver, blood flowing from the portal vein through hepatic sinusoids to the hepatic veins is filtrated through fenestrated endothelium and the space of Disse, allowing extensive metabolic exchange with lobular hepatocytes (Figure 5.1.1). When fibrosis progresses to cirrhosis (i.e. grade 4 fibrosis), this normal liver architecture is lost and becomes replaced with regenerative nodules of hepatocytes surrounded by extensive scar tissue in the space of Disse, shunting blood from hepatocytes and impairing liver function (9). In addition, the scarring together with the formation of several cytokines and vasoactive substances leads to an increased intrahepatic vascular resistance, and hence increased venous pressure in the portal system, called portal hypertension $(10,11)$.

Impairment of liver function can lead to a decrease in the production of several proteins, including albumin, coagulation factors, immune globulins, and complement factors, a decrease in hepatic glycogen storage and gluconeogenesis, decreased conjugation and drainage of bilirubin, and to impaired detoxification of blood. In 
addition, portal hypertension may lead to the development of oesophageal varices, ascites, thrombopenia due to splenomegaly, and enhanced bacterial translocation $(10,11)$. With this combination of pathophysiological changes, major complications of cirrhosis are life-threatening (variceal) bleeding, hepatic encephalopathy, hypoglycaemia, renal failure, and bacterial infections, including spontaneous bacterial peritonitis (SBP), a common and dangerous infection of ascites (12-15). Finally, liver cirrhosis may lead to the development of hepatocellular carcinoma (HCC) (16).

A

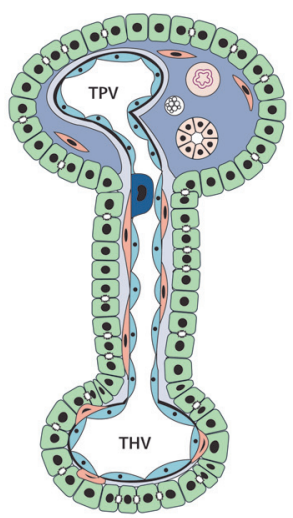

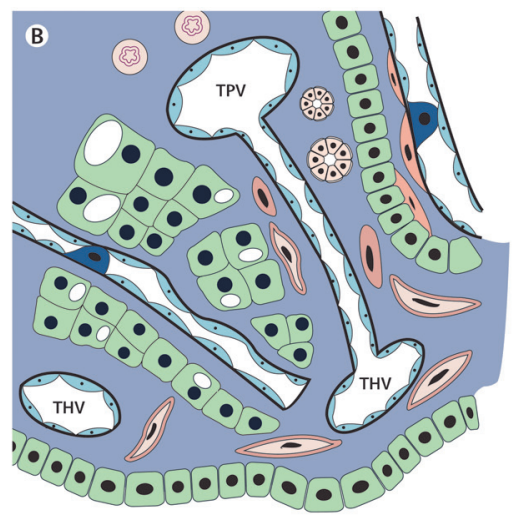

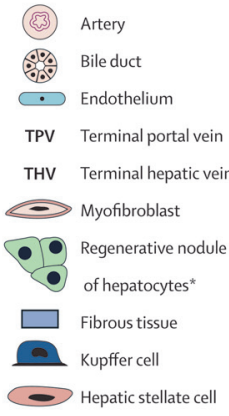

Figure 5.1.1. Panel A: Healthy liver: Mesenteric blood from the portal vein and arterial blood from branches of the hepatic artery into terminal portal veins (TPV) runs through hepatic sinusoids, where fenestrated endothelium in loose connective tissue (space of Disse) allows for extensive metabolic exchange with the lobular hepatocytes below. Sinusoidal blood is collected by terminal hepatic venules (THV) that empty into one of the three hepatic veins and finally the caval vein. Panel B: Cirrhotic liver: activated myofibroblasts that derive from perisinusoidal hepatic stellate cells and portal or central-vein fibroblasts proliferate and produce excess extracellular matrix (ECM). This event leads to fibrous portal tract expansion, central vein fibrosis and capillarisation of the sinusoids, characterised by loss of endothelial fenestrations, congestion of the space of Disse with ECM, and separation or encasement of hepatocyte islands from sinusoidal blood flow by collagenous septa. Blood is directly shunted from terminal portal veins and arteries to central veins, with consequent (intrahepatic) portal hypertension and compromised liver synthetic function. Adapted from Schuppan et al. (1).

Because of all these complications, patients with cirrhosis generally have a poor prognosis. Six-year survival of patients with decompensated cirrhosis is only $24 \%$, compared with $54 \%$ for compensated (asymptomatic / stable) cirrhosis (17). Another study reported a ten-year survival of compensated cirrhosis of $47 \%$ (18). Because of this high mortality, several prognostic scores have been developed to estimate survival and/or assess the severity of liver cirrhosis (19). The first and most widely accepted prognostic score for classification of patients with cirrhosis is the Child-Pugh score 
(Table 5.1.1), a set of 5 clinically relevant prognostic variables that were put together into a score, and validated in several cohorts of patients with cirrhosis $(19,20)$. For example, one-year survival rates for patients with Child-Pugh classes A, B and C are $100 \%, 80 \%$, and $45 \%$, respectively (21). More recently, other prognostic scores have been developed by statistical modelling of independent predictors of survival in prospective cohort studies, of which the Model for End-stage Liver Disease (MELD) score is proposed as the most promising alternative to the Child-Pugh score $(19,22)$. However, since both scores have similar prognostic accuracy, but limitations of their own, they are currently used together in clinical practice.

Treatment of liver cirrhosis usually consists of treatment of the underlying liver disease. Patients with alcoholic cirrhosis should discontinue any use of alcohol. Patients with NAFLD/NASH usually should lose weight, although the effect of weight loss on liver fibrosis is not yet clear in these patients (23). Patients with compensated cirrhosis and active hepatitis $\mathrm{B}$ or $\mathrm{C}$ can be treated with antiviral medication, leading eventually to reversal of cirrhosis in some of these patients (1). In addition, several supportive treatments have been developed to prevent and treat the major complications of liver cirrhosis $(11,12,14,24,25)$. Despite all these strategies, bacterial infections and associated hepatic decompensation remain an important problem and major cause of death in these patients. The only and ultimate treatment for liver cirrhosis is liver transplantation (1), but there is a great scarcity of livers, as only 100 liver transplantations per year were carried out in the Netherlands in 2011(26).

Table 5.1.1 Child-Pugh score for liver chirrhosis.

\begin{tabular}{lccc}
\hline & $\mathbf{1}$ point & 2 points & 3 points \\
\hline Bilirubin $(\mu \mathrm{mol} / \mathrm{I})$ & $<34$ & $34-51$ & $>51$ \\
Albumin $(\mathrm{g} / \mathrm{I})$ & $>35$ & $28-35$ & $<28$ \\
Ascites & no & mild & severe \\
Encephalopathy & no & mild & severe \\
INR & $<1.7$ & $1.7-2.30$ & $>2.30$ \\
\hline
\end{tabular}

Based on the allocation of points for all 5 variables above, a sum score is calculated between 5 and 15 . These sum scores are then divided into three Child-Pugh classes with worsening prognosis: A (5-6), B (7-9), and C (10-15). INR: international normalized ratio.

\section{Diabetes mellitus in patients with liver cirrhosis}

In patients with liver cirrhosis, disorders of glucose metabolism are very common, with up to $96 \%$ of patients having some form of glucose intolerance, and $30-50 \%$ clinical T2DM $(27,28)$. There are two major pathophysiological explanations for this high prevalence. On the one hand, obesity, the metabolic syndrome, and T2DM may eventually lead to cirrhosis, explaining why a large proportion of patients with cirrhosis have T2DM on diagnosis of cirrhosis (6). On the other hand, and far less known, is the 
so-called "hepatogenic" diabetes, which is considered a complication of chronic liver disease itself, regardless of the cause (alcoholic, viral, auto-immune, cryptogenic) $(27,29)$.

\section{The metabolic syndrome, diabetes mellitus, non-alcoholic fatty liver disease, and cirrhosis}

T2DM is present in $40-50 \%$ of patients with liver cirrhosis due to NASH $(30,31)$. The other way around, $80 \%$ of subjects with T2DM and obesity have non-alcoholic hepatic steatosis (NAFLD), and $20 \%$ have NASH $(6,32)$. NAFLD and NASH are therefore considered to be the hepatic component of the metabolic syndrome. The overall prevalence of NAFLD in the U.S. is $30 \%$, of which one third has signs of NASH. NASH leads to cirrhosis in $20 \%$ of patients during 10 years follow up. The pathogenesis of NASH is characterized by central obesity and insulin resistance in adipose tissue, muscle and liver. Figure 5.1.2 shows the effects of insulin under normal, physiological conditions; Figure 5.1.3 shows the changes that occur in insulin resistance.

The primary cause of NASH is still subject to discussion. However, it is clear that obesity is characterized by large, dysfunctional adipocytes, in which suppression of lipolysis by insulin is impaired. Consequently, circulating levels of non-esterified free fatty acids (NEFAs or FFAs) are higher in obese subjects compared with non-obese subjects $(6,33)$. In addition, adipose tissue in obesity reflects a major source of circulating inflammatory cytokines such as tumour necrosis factor- $\alpha$ (TNF- $\alpha$ ) (34). The increased levels of NEFAs and cytokines are believed to impair insulin-mediated glucose uptake in human muscle through both direct and indirect effects on insulin signal transduction (6,35-37). Beta cells in the pancreas respond to the subsequent (postprandial) hyperglycaemia by secreting more insulin to overcome the insulin resistance (33). Also, a reduced hepatic clearance of insulin may also contribute to the hyperinsulinaemia in insulin resistance (38).

The increased delivery of NEFAs and glucose to the liver combined with hyperinsulinaemia causes intrahepatic triglyceride accumulation and hepatic insulin resistance $(35,39)$. These NEFAs are processed for energy through regular $\beta$-oxidation in mitochondria and through $\omega$-oxidation in microsomes, by which oxygen radicals are created (40). Excessive oxidation of NEFAs or primary defects in oxidative phosphorylation leads to increased free radicals, hepatocellular damage and inflammation, characterized by increased expression of cytokines such as TNF- $\alpha$, transforming growth factor- $\beta$ (TGF- $\beta$ ) and interleukin-6 (IL-6) $(6,40)$. TNF- $\alpha$ induces apoptosis and necrosis of hepatocytes and, together with TGF- $\beta$, stimulates hepatic stellate cells, eventually leading to fibrosis $(28,37,40)$. Hyperinsulinaemia along with hyperglycaemia itself may also aggravate fibrosis and cirrhosis of the liver, presumably by stimulation of hepatic stellate cells $(6,37,41)$. 


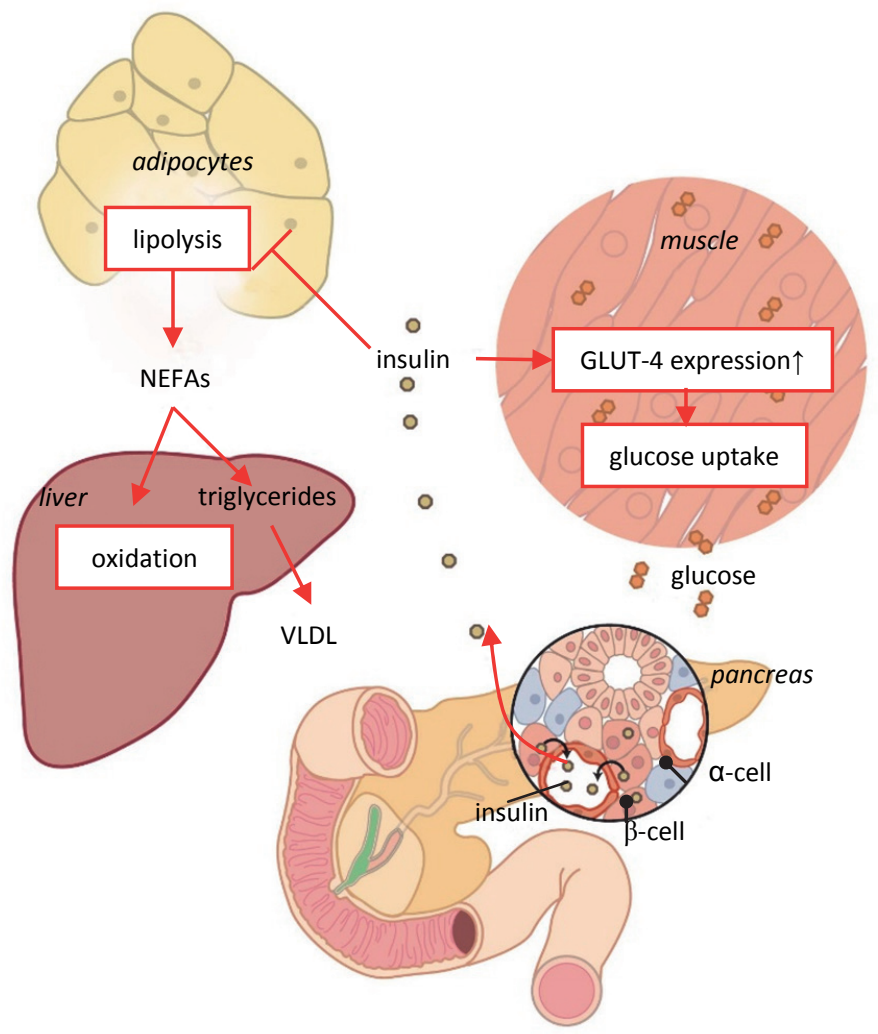

Figure 5.1.2 Insulin is secreted by beta-cells in the pancreas in response to plasma glucose levels. After binding to its receptor, insulin increases glucose transporter-4 (GLUT-4) expression in muscle cells, and thereby increases (postprandial) glucose uptake. In addition, insulin promotes storage of non-esterified fatty acids (NEFAs) and triglycerides in adipocytes and the liver by inhibiting lipolysis in adipocytes and decreasing the output of very-low-density-lipoproteins (VLDL) in the liver.

\section{Diabetes mellitus in alcoholic and viral cirrhosis}

T2DM is not only very common in patients with cirrhosis due to NAFLD/NASH, but also in patients with alcoholic or viral cirrhosis. Overall, the prevalence of T2DM among patients with cirrhosis is estimated around $20-40 \%(27,42-48)$. This is about 5 times higher compared with the general age-matched population $(43,47,49)$. However, it is unclear whether this association is independent of classical risk factors for T2DM like age, a positive family history of diabetes, or obesity, even though metabolic changes in liver disease are characterized by an insulin resistant state similar to patients with T2DM (see below). It should be noted that, in the majority of patients (50-75\%), T2DM was already known or diagnosed at the diagnosis of cirrhosis $\%(27,44,46)$. 


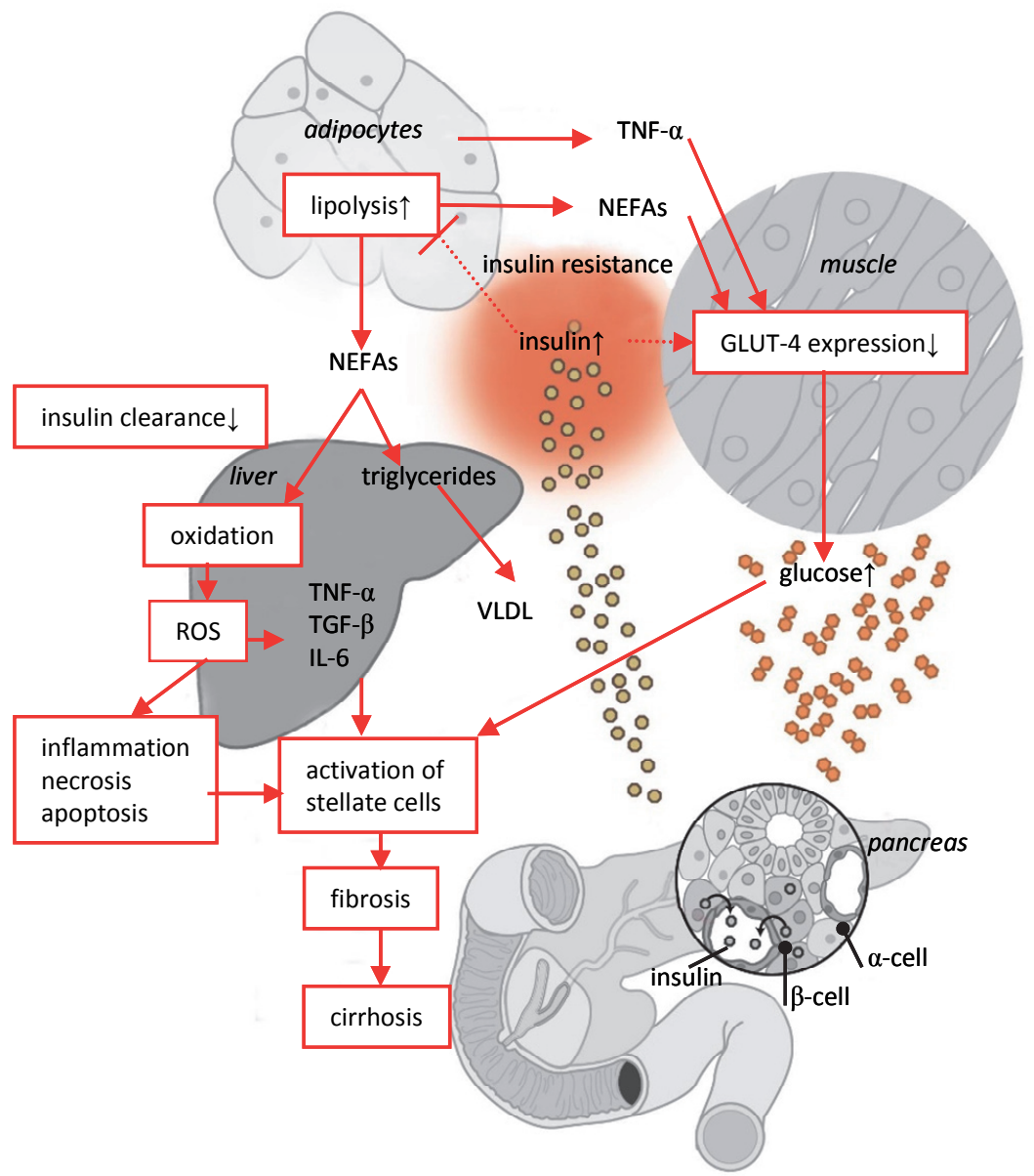

Figure 5.1.3 In an insulin-resistant state, a reduced insulin-mediated suppression of lipolysis causes increased circulating non-esterified fatty acids (NEFAs). NEFAs and adipocyte-derived cytokines such as tumour necrosis factor- $\alpha$ impair insulin signalling and insulin-mediated glucose transporter-4 (GLUT-4) expression in muscle, leading to hyperglycaemia. Glucose and NEFAs are diverted to the liver, and stored as triglycerides in the face of compensatory hyperinsulinaemia. Microsomal, mitochondrial, and peroxisomal oxidation of NEFAs leads to formation of reactive oxygen species (ROS), inducing inflammation, hepatocellular damage, apoptosis and necrosis. Under these circumstances, hepatic stellate cells are stimulated to produce collagen, leading to liver fibrosis and eventually cirrhosis. TNF- $\alpha$ : tumour necrosis factor- $\alpha$; TGF- $\beta$ : transforming growth factor- $\beta$; IL-6: interleukin-6; VLDL: very-low-density-lipoprotein.

Possibly, a portion of these patients (i.e. the patients with cryptogenic cirrhosis) may have developed cirrhosis due to NASH (in the context of the metabolic syndrome), but characteristics of NASH (micro-/macrovesicular steatosis, ballooning degeneration of hepatocytes, Mallory bodies, necroinflammation) are not always present in liver 
biopsies of advanced cirrhosis $(6,31,44,45)$. In addition, both (compensated) cirrhosis and T2DM may only be detected after a subclinical period of 5-10 years after their onset $(17,18,50)$, and, therefore, it is difficult to definitively establish the time course of their relationship in human studies. As such, it remains unclear whether classical risk factors explain the high prevalence of T2DM in patients with cirrhosis, or whether the liver disease itself causes alternations in glucose metabolism.

To date, only one observational cohort study has established that T2DM develops in a considerable portion of patients after the diagnosis of cirrhosis has been established (48). This observational cohort study consisted of 100 patients with alcoholic or viral cirrhosis with normal glucose tolerance at baseline. Severity of liver cirrhosis at baseline was classified as Child-Pugh score A in all patients. Liver cirrhosis deteriorated during 4 years follow-up in 34 patients, and was classified as Child-Pugh score B or C. During the follow up, $21 \%$ of patients with compensated cirrhosis (Child-Pugh A) and $35 \%$ of patients with progressive cirrhosis (Child-Pugh B or C) developed diabetes mellitus de novo, as diagnosed by an oral glucose tolerance test. No differences in diabetes incidence were observed between sexes, between positive or negative family history for T2DM, or between the aetiology of cirrhosis.

The pathophysiology of diabetes mellitus associated with chronic liver disease is complex and not yet fully understood. Similarly to T2DM in obesity, both peripheral insulin resistance in muscle and fat, and beta cell failure seem to be involved. Even normoglycaemic patients with alcoholic or viral cirrhosis show a reduced total glucose disposal during hyperinsulinaemic euglycaemic clamp studies compared with healthy control subjects (e.g. $6.09 \mathrm{mg} / \mathrm{kg} / \mathrm{min}$ vs. $7.95 \mathrm{mg} / \mathrm{kg} / \mathrm{min}$; $\mathrm{p}<0.01)(29,42,51,52)$, as well as reduced glycogen deposition in muscle during the clamp $(8.6 \mathrm{mg} / \mathrm{mg}$ protein vs. $12.0 \mathrm{mg} / \mathrm{mg}$ protein; $\mathrm{p}<0.05$ ) (52). This reduced glucose disposal in patients with cirrhosis has also been linked to a reduced uptake of glucose in skeletal muscle by positron emission tomography (PET) (53). When patients with cirrhosis develop impaired glucose tolerance (IGT) and T2DM, total glucose disposal becomes further reduced to $3.55 \mathrm{mg} / \mathrm{kg} / \mathrm{min}$ and $4.44 \mathrm{mg} / \mathrm{kg} / \mathrm{min}$ respectively ( $p<0.01$ vs. controls) $(29,51)$. Additionally, patients with cirrhosis and insulin resistance have compensatory hyperinsulinaemia (16-17 $\mu \mathrm{U} / \mathrm{ml})$, similar to obese, insulin-resistant subjects.

Pro-inflammatory cytokines such as TNF- $\alpha$ en IL- 6 from the liver are a possible cause of insulin resistance in liver diseases, since higher circulating levels of these cytokines are observed in patients with alcoholic or viral hepatitis and cirrhosis (37,54-56). Although studies using muscle biopsies have not been performed in patients with liver disease, this hypothesis is very plausible, because these cytokines are known for their impairment of insulin signalling in muscle as described above $(36,37)$, and serum levels have been shown to correlate with homeostasis model assessment insulin resistance (HOMA-IR) in patients with chronic hepatitis C (56). Furthermore, the incidence of impaired fasting glucose and T2DM in patients with chronic hepatitis $C$ was reduced in patients with a sustained virological response after treatment vs. those without, 
indicating that clearance of the virus and associated inflammation may improve insulin resistance (57).

Besides inflammatory cytokines, chronic hyperinsulinaemia itself may also aggravate insulin resistance leading to a vicious circle. Hyperinsulinaemia in liver disease can be attributed to a compensatory hypersecretion by beta cells ( $49 \%$ higher secretion than in healthy controls), but also to a reduced insulin clearance by the cirrhotic liver (e.g. $725 \mathrm{ml} / \mathrm{min} / \mathrm{m}^{2}$ in patients with cirrhosis vs. $1165 \mathrm{ml} / \mathrm{min} / \mathrm{m}^{2}$ in controls; $p<0.05)$ due to portosystemic shunting of blood $(51,58,59)$. Prolonged reduction of this hyperinsulinaemia for 4 days by continuous infusion of a somatostatinanalogue normalized insulin-mediated glucose disposal to values not significantly different from healthy subjects (from $5.75 \mathrm{mg} / \mathrm{kg} / \mathrm{min}$ before infusion to $7.63 \mathrm{mg} / \mathrm{kg} / \mathrm{min}$ after 4 days of somatostatinanalogue) (60), supporting the hypothesis that chronic hyperinsulinaemia contributes to insulin resistance in patients with cirrhosis.

A difference with T2DM in obesity is that hepatic insulin resistance is not yet present at the time of glucose intolerance in patients with alcoholic and viral cirrhosis, and endogenous glucose production by the liver is still adequately suppressed by insulin $(29,51)$. A possible explanation could be that inflammatory cytokines mainly affect insulin signalling in peripheral muscle and fat, and not in the liver (61). Insulin resistance in adipose tissue leads to higher fasting NEFA concentrations in patients with cirrhosis compared with healthy controls ( $933 \mu \mathrm{mol} / \mathrm{I}$ vs. $711 \mu \mathrm{mol} / \mathrm{l})$, since suppression of lipolysis by insulin is attenuated (e.g. suppression of NEFAs by low dose insulin infusion to $50 \%$ of basal in patients with cirrhosis vs. suppression to $20 \%$ in healthy controls; $p<0.01)(51,62)$. Because of the impaired suppression of lipolysis in patients with liver disease, increased delivery of NEFAs to the liver also leads to hepatic steatosis in these patients.

An essential step in the pathogenesis of T2DM is pancreatic beta cell dysfunction (Figure 5.1.4) $(29,42)$. In the case of prolonged or progressive insulin resistance, beta cells can become unable to respond to elevations in plasma glucose with sufficient insulin secretion $(47 \%$ lower insulin secretion than in healthy controls) $(29,42,58)$. The mechanisms responsible for beta cell dysfunction are not yet solved, but genetic factors, excessive alcohol consumption, and chronic postprandial hyperglycaemia (glucotoxicity) may all contribute to this.

Altogether, both pathophysiological theories for the high prevalence of T2DM in patients with cirrhosis share some key aspects (inflammation, NEFAs). Therefore, it would be interesting to examine, in an epidemiological study, whether the liver disease itself (cirrhosis) is independently associated with (the development of) T2DM, or whether this association can be explained by classical risk factors. 


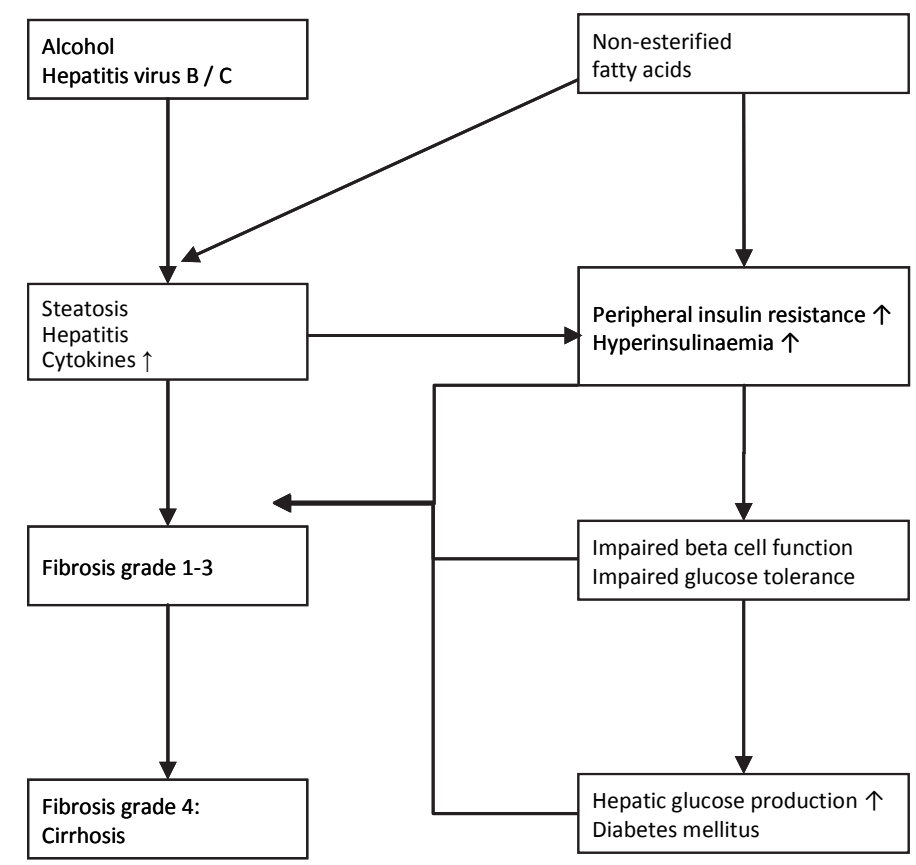

Figure 5.1.4 Pathogenesis of diabetes mellitus in patients with liver cirrhosis. Adipocyte dysfunction in obesity leads to increased circulating non-esterified fatty acids (NEFAs) and proinflammatory cytokines. The abundant flux of NEFAs to the liver may lead to steatosis, steatohepatitis, and eventually cirrhosis. Chronic hepatocellular injury, due to alcohol abuse or chronic viral hepatitis, also leads to increased circulating cytokine levels. Both cytokines and NEFAs can cause insulin resistance, which is first compensated by hyperinsulinaemia, but later followed by impaired glucose tolerance and eventually diabetes mellitus. Both hyperglycaemia and hyperinsulinaemia may stimulate hepatic stellate cells, and hence contribute to a more rapid progression of liver diseases.

\section{Prognostic value of diabetes complicating cirrhosis}

As described earlier in this thesis, mortality among patients with T2DM in the general population is on average 1.4 times higher compared with subjects without T2DM, particularly due to the increased risk of cardiovascular disease (63). However, patients with cirrhosis have a shorter life expectancy $(17,18,21)$. Therefore, an interesting question, given its high prevalence, may be whether T2DM is also a prognostic factor in these subjects. T2DM is neither included in the Child-Pugh score, nor in the model for end-stage liver disease (MELD)-score $(19,20,22)$, but some studies have suggested that T2DM may be a risk factor for overall mortality. Ten-year survival in a cohort of 361 patients with cirrhosis was $17 \%$ in patients with T2DM vs. $32 \%$ in patients without T2DM (adjusted hazard ratio: $1.18 ; 95 \% \mathrm{Cl} 1.03-1.37$ ) (64). This association was 
attenuated, however, when the presence of oesophageal varices was included as covariate in a subgroup of 271 patients. T2DM also increased mortality in 2 other small studies during one year (65) and two years of follow-up (66). In contrast, in two other prospective studies, T2DM did not influence prognosis (27), or was no longer significantly associated with 2-year mortality after adjustment for Child-Pugh score and serum creatinine (67). Finally, some studies have also investigated the prognostic role of IGT in patients with cirrhosis, but clear differences in mortality compared with normal glucose metabolism have not (yet) been observed $(27,65,68)$. It should be noted that these studies generally consisted of a small sample of patients $(n=<100)$ $(27,65,66,68)$, had a low number of events $(n=<20)(27,65,67,68)$, and/or included patients with longstanding cirrhosis at baseline $(64,67)$.

Remarkably, most patients with cirrhosis and T2DM do not die because of cardiovascular complications, but due to liver failure or complications such as variceal bleeding, hepatic encephalopathy, hepatorenal syndrome, hepatocellular carcinoma or spontaneous bacterial peritonitis $(27,64,66)$. Moreover, the prevalence of micro- and macrovascular complications in patients with cirrhosis and T2DM is comparable with healthy subjects, and lower than in patients with T2DM only $(27,69,70)$. This can be explained by the relatively short duration of diabetes in patients with cirrhosis and their apparently protective cardiovascular risk profile, consisting of low vascular resistance, thrombocytopenia, high bilirubin, low levels of cholesterol and lipids, and decreased production of coagulation factors $(69,70)$. As such, a considerable proportion of patients with cirrhosis and T2DM are expected never to develop cardiovascular complications because of the reduced life expectancy due to the cirrhosis itself.

The increased mortality in patients with cirrhosis and T2DM could theoretically be explained by an increased susceptibility to bacterial infections, as has been reported in patients with T2DM in general $(28,71,72)$. Patients with cirrhosis are characterized by immune dysfunction, which makes them highly susceptible to a variety of (bacterial) infections such as respiratory tract infections, urinary tract infections, skin infections, (abdominal) sepsis, and spontaneous bacterial peritonitis (SBP) $(73,74)$. As such, bacterial infections have been reported in about one third of patients with cirrhosis admitted to a hospital, either on admission, or during the hospital stay (15). Such infections are associated with a four-fold increased mortality, and represent one of the most common causes of death in patients with cirrhosis (13). Currently, only a higher Child-Pugh score (or MELD score) is an established risk factor for infections $(73,75)$. Whether the presence of T2DM in patients with cirrhosis further increases the risk of infections, is yet unclear, although a higher prevalence of bacteriuria has been reported in cirrhotic patients with T2DM versus those without (76). In addition, a few small case-control studies on subjects with ascites showed higher rates of T2DM in patients with SBP compared to those with sterile ascites, although not statistically significant $(75,77,78)$. Finally, T2DM has recently been associated with SBP resistant to 
third-generation cephalosporins (79), which could reflect several previous courses of antibiotics that have been given to these patients for other infections.

Besides a potentially increased risk of infections in patients with T2DM, the pathophysiological interaction between insulin resistance, hepatic steatosis and inflammation may imply that liver function may deteriorate more quickly in the presence of IGT or T2DM (Figure 5.1.4). Insulin resistance, calculated by homeostasis model assessment for insulin resistance (HOMA-IR), is indeed correlated with the degree of fibrosis in liver biopsies of patients with viral hepatitis (odds ratio for higher stage of fibrosis: 1.3 per unit increase in HOMA-IR; 95\%Cl 1.1-1.4) (80). In addition, $2 \mathrm{~h}$ plasma glucose levels have also been linked to the degree of fibrosis in NASH/NAFLD (30), and the presence of T2DM was associated with the degree of fibrosis in hereditary haemochromatosis (81). In patients with cirrhosis (i.e. fibrosis grade 4), the presence of T2DM has also been associated with the Child-Pugh score at baseline $(46,47)$, or a deterioration during follow up (48), but other studies have not confirmed this association $(65,67)$.

In conclusion, the prognosis of cirrhosis is poor, and the prognostic impact of T2DM in patients with liver cirrhosis is still unclear. Therefore, it seems relevant to investigate the potential prognostic impact of T2DM in patients with cirrhosis. In addition, such research may also improve our understanding on treatment of T2DM in these patients (e.g. whether strict glycaemic control is indicated).

\section{Current treatment of diabetes in patients with cirrhosis}

At this moment, it is unclear whether treating T2DM decreases mortality and morbidity of patients with cirrhosis. Antihyperglycaemic treatment may theoretically reduce the incidence of infections and reduce mortality, but there have been no randomized controlled trials on treatment of T2DM in patients with cirrhosis. The effect of treatment of T2DM on mortality has been examined in one retrospective study of patients with cirrhosis (82). Five-year mortality of patients with a mean serum glucose $>7.0 \mathrm{mmol} / \mathrm{l}$ was $73 \%$ compared with $21 \%$ in patients with mean serum glucose levels $\leq 7.0 \mathrm{mmol} / \mathrm{l}(\mathrm{p}=0.006)$. However, it was not clearly discussed by the authors how these mean glucose values were obtained. Moreover, the effect was only observed in patients with cirrhosis due to hepatitis $C$, and not for hepatitis B. In another cohort study of 100 patients with cirrhosis due to hepatitis $C$ and T2DM, metformin use ( $n=26$ patients) was associated with a decreased incidence of hepatocellular carcinoma, and also with decreased liver-related mortality and/or need for liver transplantation (83).

Current guidelines for treatment of T2DM cannot simply be used in this specific patient group because of liver failure and possible continuation of alcohol abuse. However, in obese patients with T2DM and compensated cirrhosis, regular physical activity and weight loss may be just as important to increase insulin sensitivity as in obese patients with T2DM without liver disease $(6,28)$. 
In more advanced stages of cirrhosis and in case of malnutrition, pharmacological treatment may be the only option. Metformin, sulfonylurea derivatives and thiazolidinediones are relatively contraindicated due to a reduced hepatic clearance. In case the physician wants to prescribe an oral antihyperglycaemic drug, short-acting agents such as gliclazide or repaglinide are preferred $(28,84)$. Acarbose has been shown to effectively reduce fasting and postprandial hyperglycaemia in patients with cirrhosis, as well as improve blood ammonia levels and symptoms of hepatic encephalopathy (85). In addition, short-acting insulin injections are preferred when insulin therapy is the only therapeutic option. Individual insulin requirements may differ between patients when on insulin therapy, but are often lower in patients with decompensated cirrhosis compared with compensated cirrhosis because of impaired hepatic clearance. Moreover, the capacity of the liver to maintain sufficiently high fasting blood glucose by glycogenolysis and gluconeogenesis may be severely impaired in decompensated cirrhosis (84). Together with a reduced insulin clearance, this may often lead to hypoglycaemia. Because of this high risk of hypoglycaemia, frequent blood glucose monitoring is indispensable in the treatment of T2DM in patients with cirrhosis (28). Since patients with cirrhosis and T2DM often die from hepatic complications, but show a favourable cardiovascular risk profile, treatment of cardiovascular risk factors has lower priority $(69,84)$.

\section{Outline and aims of this chapter}

T2DM is very common among patients with liver cirrhosis and develops in a state of inflammation and insulin resistance. Because of the complex interaction between both diseases, it is clinically difficult to determine what is cause and what is consequence. Moreover, it remains unclear whether classical risk factors explain the high prevalence of T2DM in patients with cirrhosis, or whether the liver disease itself causes alternations in glucose metabolism. For this reason, we compared the prevalence of T2DM in a cohort of patients with cirrhosis and healthy controls, and examined whether the association between cirrhosis and T2DM was independent of classical risk factors (Chapter 5.2). In addition, it is still unclear whether the presence of T2DM is associated with increased mortality in patients with cirrhosis. From a pathophysiological point of view, the prognosis of patients with both cirrhosis and T2DM could be worse because of a more rapid deterioration of liver function and increased susceptibility for infections. Therefore, we investigated, in a relatively large cohort, whether the presence of T2DM at diagnosis of cirrhosis was associated with increased overall mortality and liver-related mortality (Chapter 5.3). In this chapter, we also examined whether T2DM was associated with incident SBP, a common and serious infection in patients with cirrhosis, to test the hypothesis that T2DM may increase the susceptibility to infections. 


\section{References}

1. Schuppan D, Afdhal NH: Liver cirrhosis. Lancet 2008;371:838-851

2. Roberts SE, Goldacre MJ, Yeates D: Trends in mortality after hospital admission for liver cirrhosis in an English population from 1968 to 1999 . Gut 2005;54:1615-1621

3. Leon DA, McCambridge J: Liver cirrhosis mortality rates in Britain from 1950 to 2002: an analysis of routine data. Lancet 2006;367:52-56

4. El-Serag HB: Epidemiology of viral hepatitis and hepatocellular carcinoma. Gastroenterology 2012;142:1264-1273 e1261

5. Corrao G, Zambon A, Torchio P, Arico S, La Vecchia C, di Orio F: Attributable risk for symptomatic liver cirrhosis in Italy. Collaborative Groups for the Study of Liver Diseases in Italy. J Hepatol 1998;28: 608-614

6. Farrell GC, Larter CZ: Nonalcoholic fatty liver disease: from steatosis to cirrhosis. Hepatology 2006;43:S99-S112

7. Clark JM: The epidemiology of nonalcoholic fatty liver disease in adults. J Clin Gastroenterol 2006;40 Suppl 1:S5-10

8. Bircher J BJ, Mclntyre N, Rizzetto M, Rodes J, eds.: Oxford textbook of clinical hepatology, 2nd edn. Oxford, Oxford University Press, 1999

9. Schaffner F, Poper H: Capillarization of hepatic sinusoids in man. Gastroenterology 1963;44:239-242

10. Garcia-Pagan JC, Gracia-Sancho J, Bosch J: Functional aspects on the pathophysiology of portal hypertension in cirrhosis. J Hepatol 2012;57:458-461

11. Sanyal AJ, Bosch J, Blei A, Arroyo V: Portal hypertension and its complications. Gastroenterology 2008;134:1715-1728

12. Garcia-Tsao G: Current management of the complications of cirrhosis and portal hypertension: variceal hemorrhage, ascites, and spontaneous bacterial peritonitis. Gastroenterology 2001;120:726-748

13. Arvaniti V, D'Amico G, Fede G, Manousou P, Tsochatzis E, Pleguezuelo M, Burroughs AK: Infections in patients with cirrhosis increase mortality four-fold and should be used in determining prognosis. Gastroenterology 2010;139:1246-1256, 1256 e1241-1245

14. Fernandez J, Gustot T: Management of bacterial infections in cirrhosis. J Hepatol 2012;56 Suppl 1:S1-12

15. Fernandez J, Navasa M, Gomez J, Colmenero J, Vila J, Arroyo V, Rodes J: Bacterial infections in cirrhosis: epidemiological changes with invasive procedures and norfloxacin prophylaxis. Hepatology 2002;35: 140-148

16. Fattovich G, Stroffolini T, Zagni I, Donato F: Hepatocellular carcinoma in cirrhosis: incidence and risk factors. Gastroenterology 2004;127:S35-50

17. D'Amico G, Morabito A, Pagliaro L, Marubini E: Survival and prognostic indicators in compensated and decompensated cirrhosis. Dig Dis Sci 1986;31:468-475

18. Gines P, Quintero E, Arroyo V, Teres J, Bruguera M, Rimola A, Caballeria J, Rodes J, Rozman C: Compensated cirrhosis: natural history and prognostic factors. Hepatology 1987;7:122-128

19. Durand F, Valla D: Assessment of the prognosis of cirrhosis: Child-Pugh versus MELD. J Hepatol 2005;42 Suppl:S100-107

20. Pugh RN, Murray-Lyon IM, Dawson JL, Pietroni MC, Williams R: Transection of the oesophagus for bleeding oesophageal varices. Br J Surg 1973;60:646-649

21. Infante-Rivard C, Esnaola S, Villeneuve JP: Clinical and statistical validity of conventional prognostic factors in predicting short-term survival among cirrhotics. Hepatology 1987; 7:660-664

22. Kamath PS, Wiesner RH, Malinchoc M, Kremers W, Therneau TM, Kosberg CL, D'Amico G, Dickson ER, Kim WR: A model to predict survival in patients with end-stage liver disease. Hepatology 2001;33: 464-470

23. De Ridder RJ, Schoon EJ, Smulders JF, van Hout GC, Stockbrugger RW, Koek GH: Review article: Nonalcoholic fatty liver disease in morbidly obese patients and the effect of bariatric surgery. Aliment Pharmacol Ther 2007;26 Suppl 2:195-201

24. European Association for the study of the Liver, EASL clinical practice guidelines on the management of ascites, spontaneous bacterial peritonitis, and hepatorenal syndrome in cirrhosis. J Hepatol 2010;53:397-417 
25. Gines P, Fernandez J, Durand F, Saliba F: Management of critically-ill cirrhotic patients. J Hepatol 2012;56 Suppl 1:S13-24

26. Jaarverslag 2011 Nederlandse Transplantatiestichting [article online], 2011. Available from http://www.transplantatiestichting.nl/sites/default/files/product/downloads/2729_2_nts_jaarverslag_ 2011_1.pdf.

27. Holstein A, Hinze S, Thiessen E, Plaschke A, Egberts EH: Clinical implications of hepatogenous diabetes in liver cirrhosis. J Gastroenterol Hepatol 2002;17:677-681

28. Garcia-Compean D, Jaquez-Quintana JO, Gonzalez-Gonzalez JA, Maldonado-Garza H: Liver cirrhosis and diabetes: risk factors, pathophysiology, clinical implications and management. World J Gastroenterol 2009;15:280-288

29. Petrides AS, Vogt C, Schulze-Berge D, Matthews D, Strohmeyer G: Pathogenesis of glucose intolerance and diabetes mellitus in cirrhosis. Hepatology 1994;19:616-627

30. Wong VW, Hui AY, Tsang SW, Chan JL, Wong GL, Chan AW, So WY, Cheng AY, Tong PC, Chan FK, Sung JJ, Chan HL: Prevalence of undiagnosed diabetes and postchallenge hyperglycaemia in Chinese patients with non-alcoholic fatty liver disease. Aliment Pharmacol Ther 2006;24:1215-1222

31. Caldwell SH, Oelsner DH, lezzoni JC, Hespenheide EE, Battle EH, Driscoll CJ: Cryptogenic cirrhosis: clinical characterization and risk factors for underlying disease. Hepatology 1999;29:664-669

32. Thewissen CM, Tiebosch AT, Kloppenburg WD, Haagsmna EB, Hoogenberg K: [Liver cirrhosis in diabetes mellitus; a complication not always recognized]. Ned Tijdschr Geneeskd 2008;152:2369-2374

33. DeFronzo RA, Tripathy D: Skeletal muscle insulin resistance is the primary defect in type 2 diabetes. Diabetes Care 2009;32 Suppl 2:S157-163

34. Hotamisligil GS, Arner P, Caro JF, Atkinson RL, Spiegelman BM: Increased adipose tissue expression of tumor necrosis factor-alpha in human obesity and insulin resistance. J Clin Invest 1995;95:2409-2415

35. Samuel VT, Petersen KF, Shulman GI: Lipid-induced insulin resistance: unravelling the mechanism. Lancet 2010;375:2267-2277

36. Tanti JF, Jager J: Cellular mechanisms of insulin resistance: role of stress-regulated serine kinases and insulin receptor substrates (IRS) serine phosphorylation. Curr Opin Pharmacol 2009;9:753-762

37. Picardi A, D'Avola D, Gentilucci UV, Galati G, Fiori E, Spataro S, Afeltra A: Diabetes in chronic liver disease: from old concepts to new evidence. Diabetes Metab Res Rev 2006;22:274-283

38. Kotronen A, Juurinen L, Tiikkainen M, Vehkavaara S, Yki-Jarvinen H: Increased liver fat, impaired insulin clearance, and hepatic and adipose tissue insulin resistance in type 2 diabetes. Gastroenterology 2008;135:122-130

39. Korenblat KM, Fabbrini E, Mohammed BS, Klein S: Liver, muscle, and adipose tissue insulin action is directly related to intrahepatic triglyceride content in obese subjects. Gastroenterology 2008;134: 1369-1375

40. Tessari P, Coracina A, Cosma A, Tiengo A: Hepatic lipid metabolism and non-alcoholic fatty liver disease. Nutr Metab Cardiovasc Dis 2009;19:291-302

41. Paradis V, Perlemuter G, Bonvoust F, Dargere D, Parfait B, Vidaud M, Conti M, Huet S, Ba N, Buffet C, Bedossa P: High glucose and hyperinsulinemia stimulate connective tissue growth factor expression: a potential mechanism involved in progression to fibrosis in nonalcoholic steatohepatitis. Hepatology 2001;34:738-744

42. Megyesi C, Samols E, Marks V: Glucose tolerance and diabetes in chronic liver disease. Lancet 1967;2:1051-1056

43. Custro N, Carroccio A, Ganci A, Scafidi V, Campagna P, Di Prima L, Montalto G: Glycemic homeostasis in chronic viral hepatitis and liver cirrhosis. Diabetes Metab 2001;27:476-481

44. Poonawala A, Nair SP, Thuluvath PJ: Prevalence of obesity and diabetes in patients with cryptogenic cirrhosis: a case-control study. Hepatology 2000;32:689-692

45. Tellez-Avila FI, Sanchez-Avila F, Garcia-Saenz-de-Sicilia M, Chavez-Tapia NC, Franco-Guzman AM, LopezArce G, Cerda-Contreras E, Uribe M: Prevalence of metabolic syndrome, obesity and diabetes type 2 in cryptogenic cirrhosis. World J Gastroenterol 2008;14:4771-4775

46. Alavian SM, Hajarizadeh B, Nematizadeh F, Larijani B: Prevalence and determinants of diabetes mellitus among Iranian patients with chronic liver disease. BMC Endocr Disord 2004;4:4 
47. Zein NN, Abdulkarim AS, Wiesner RH, Egan KS, Persing DH: Prevalence of diabetes mellitus in patients with end-stage liver cirrhosis due to hepatitis C, alcohol, or cholestatic disease. J Hepatol 2000;32: 209-217

48. Gentile S, Loguercio C, Marmo R, Carbone L, Del Vecchio Blanco C: Incidence of altered glucose tolerance in liver cirrhosis. Diabetes Res Clin Pract 1993;22:37-44

49. Baan CA, van Baal PH, Jacobs-van der Bruggen MA, Verkley H, Poos MJ, Hoogenveen RT, Schoemaker CG: [Diabetes mellitus in the Netherlands: estimate of the current disease burden and prognosis for 2025]. Ned Tijdschr Geneeskd 2009;153:1052-1058

50. Harris MI, Klein R, Welborn TA, Knuiman MW: Onset of NIDDM occurs at least 4-7 yr before clinical diagnosis. Diabetes Care 1992;15:815-819

51. Petrides AS, Groop LC, Riely CA, DeFronzo RA: Effect of physiologic hyperinsulinemia on glucose and lipid metabolism in cirrhosis. J Clin Invest 1991;88:561-570

52. Kruszynska $Y$, Williams N, Perry $M$, Home P: The relationship between insulin sensitivity and skeletal muscle enzyme activities in hepatic cirrhosis. Hepatology 1988;8:1615-1619

53. Selberg O, Burchert W, vd Hoff J, Meyer GJ, Hundeshagen H, Radoch E, Balks HJ, Muller MJ: Insulin resistance in liver cirrhosis. Positron-emission tomography scan analysis of skeletal muscle glucose metabolism. J Clin Invest 1993;91:1897-1902

54. Tilg H, Diehl AM: Cytokines in alcoholic and nonalcoholic steatohepatitis. N Engl J Med 2000;343: 1467-1476

55. Tilg H, Wilmer A, Vogel W, Herold M, Nolchen B, Judmaier G, Huber C: Serum levels of cytokines in chronic liver diseases. Gastroenterology 1992;103:264-274

56. Lecube A, Hernandez C, Genesca J, Simo R: Proinflammatory cytokines, insulin resistance, and insulin secretion in chronic hepatitis C patients: A case-control study. Diabetes Care 2006;29:1096-1101

57. Simo R, Lecube A, Genesca J, Esteban JI, Hernandez C: Sustained virological response correlates with reduction in the incidence of glucose abnormalities in patients with chronic hepatitis $C$ virus infection. Diabetes Care 2006;29:2462-2466

58. Kruszynska YT, Goulas S, Wollen N, McIntyre N: Insulin secretory capacity and the regulation of glucagon secretion in diabetic and non-diabetic alcoholic cirrhotic patients. J Hepatol 1998;28:280-291

59. Letiexhe MR, Scheen AJ, Gerard PL, Bastens BH, Pirotte J, Belaiche J, Lefebvre PJ: Insulin secretion, clearance, and action on glucose metabolism in cirrhotic patients. J Clin Endocrinol Metab 1993;77: 1263-1268

60. Petrides AS, Stanley T, Matthews DE, Vogt C, Bush AJ, Lambeth H: Insulin resistance in cirrhosis: prolonged reduction of hyperinsulinemia normalizes insulin sensitivity. Hepatology 1998;28:141-149

61. Hotamisligil GS, Budavari A, Murray D, Spiegelman BM: Reduced tyrosine kinase activity of the insulin receptor in obesity-diabetes. Central role of tumor necrosis factor-alpha. J Clin Invest 1994;94: 1543-1549

62. Merli M, Leonetti F, Riggio O, Giaccari A, Romiti A, Sbraccia P, Tamburrano G: Resistance to insulin suppression of plasma free fatty acids in liver cirrhosis. J Endocrinol Invest 1990;13:787-795

63. de Marco R, Locatelli F, Zoppini G, Verlato G, Bonora E, Muggeo M: Cause-specific mortality in type 2 diabetes. The Verona Diabetes Study. Diabetes Care 1999;22:756-761

64. Bianchi G, Marchesini G, Zoli M, Bugianesi E, Fabbri A, Pisi E: Prognostic significance of diabetes in patients with cirrhosis. Hepatology 1994;20:119-125

65. Hagel S, Bruns T, Herrmann A, Stallmach A, Schmidt C: Abnormal glucose tolerance: a predictor of 30day mortality in patients with decompensated liver cirrhosis. Z Gastroenterol 2011;49:331-334

66. Moreau R, Delegue P, Pessione F, Hillaire S, Durand F, Lebrec D, Valla DC: Clinical characteristics and outcome of patients with cirrhosis and refractory ascites. Liver Int 2004;24:457-464

67. Quintana JO, Garcia-Compean D, Gonzalez JA, Perez JZ, Gonzalez FJ, Espinosa LE, Hernandez PL, Cabello ER, Villarreal ER, Rendon RF, Garza HM: The impact of diabetes mellitus in mortality of patients with compensated liver cirrhosis-a prospective study. Ann Hepatol 2011;10:56-62

68. Nishida T, Tsuji S, Tsujii M, Arimitsu S, Haruna Y, Imano E, Suzuki M, Kanda T, Kawano S, Hiramatsu N, Hayashi N, Hori M: Oral glucose tolerance test predicts prognosis of patients with liver cirrhosis. Am J Gastroenterol 2006;101:70-75 
69. Marchesini G, Ronchi M, Forlani G, Bugianesi E, Bianchi G, Fabbri A, Zoli M, Melchionda N: Cardiovascular disease in cirrhosis--a point-prevalence study in relation to glucose tolerance. Am J Gastroenterol 1999;94:655-662

70. Fujiwara F, Ishii M, Taneichi H, Miura M, Toshihiro M, Takebe N, Ishida W, Kaneko Y, Kato A, Suzuki K, Satoh J: Low incidence of vascular complications in patients with diabetes mellitus associated with liver cirrhosis as compared with type 2 diabetes mellitus. Tohoku J Exp Med 2005;205:327-334

71. Geerlings SE, Hoepelman Al: Immune dysfunction in patients with diabetes mellitus (DM). FEMS Immunol Med Microbiol 1999;26:259-265

72. Rajagopalan S: Serious infections in elderly patients with diabetes mellitus. Clin Infect Dis 2005;40: 990-996

73. Christou L, Pappas G, Falagas ME: Bacterial infection-related morbidity and mortality in cirrhosis. Am J Gastroenterol 2007;102:1510-1517

74. Bonnel AR, Bunchorntavakul C, Reddy KR: Immune dysfunction and infections in patients with cirrhosis. Clin Gastroenterol Hepatol 2011;9:727-738

75. Obstein KL, Campbell MS, Reddy KR, Yang YX: Association between model for end-stage liver disease and spontaneous bacterial peritonitis. Am J Gastroenterol 2007;102:2732-2736

76. Amato A, Precone DF, Carannante N, Brancaccio G, Stornaiuolo G, Galante D, Gaeta GB: [Prevalence and risk factors for bacteriuria in patients with cirrhosis]. Infez Med 2005;13:103-108

77. Bajaj JS, Zadvornova Y, Heuman DM, Hafeezullah M, Hoffmann RG, Sanyal AJ, Saeian K: Association of proton pump inhibitor therapy with spontaneous bacterial peritonitis in cirrhotic patients with ascites. Am J Gastroenterol 2009;104:1130-1134

78. Sorrentino P, Tarantino G, Conca P, Perrella A, Perrella O: Clinical presentation and prevalence of spontaneous bacterial peritonitis in patients with cryptogenic cirrhosis and features of metabolic syndrome. Can J Gastroenterol 2004;18:381-386

79. Ariza X, Castellote J, Lora-Tamayo J, Girbau A, Salord S, Rota R, Ariza J, Xiol X: Risk factors for resistance to ceftriaxone and its impact on mortality in community, healthcare and nosocomial spontaneous bacterial peritonitis. J Hepatol 2012;56:825-832

80. Hui JM, Sud A, Farrell GC, Bandara P, Byth K, Kench JG, McCaughan GW, George J: Insulin resistance is associated with chronic hepatitis $\mathrm{C}$ virus infection and fibrosis progression [corrected]. Gastroenterology 2003;125:1695-1704

81. Wood MJ, Powell LW, Dixon JL, Ramm GA: Clinical cofactors and hepatic fibrosis in hereditary hemochromatosis: The role of diabetes mellitus. Hepatology 2012;56:904-911

82. Kwon SY, Kim SS, Kwon OS, Kwon KA, Chung MG, Park DK, Kim YS, Koo YS, Kim YK, Choi DJ, Kim JH: Prognostic significance of glycaemic control in patients with HBV and HCV-related cirrhosis and diabetes mellitus. Diabet Med 2005;22:1530-1535

83. Nkontchou G, Cosson E, Aout M, Mahmoudi A, Bourcier V, Charif I, Ganne-Carrie N, Grando-Lemaire V, Vicaut $E$, Trinchet JC, Beaugrand $M$ : Impact of metformin on the prognosis of cirrhosis induced by viral hepatitis C in diabetic patients. J Clin Endocrinol Metab 2011;96:2601-2608

84. Tolman KG, Fonseca V, Dalpiaz A, Tan MH: Spectrum of liver disease in type 2 diabetes and management of patients with diabetes and liver disease. Diabetes Care 2007;30:734-743

85. Gentile S, Guarino G, Romano M, Alagia IA, Fierro M, Annunziata S, Magliano PL, Gravina AG, Torella R: A randomized controlled trial of acarbose in hepatic encephalopathy. Clin Gastroenterol Hepatol 2005; 3:184-191 


\section{Chapter}

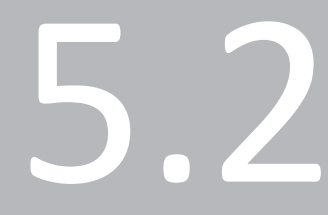

High prevalence of diabetes mellitus in patients with liver cirrhosis

Nick Wlazlo, Hanneke JBH Beijens, Erik J Schoon, Hans P Sauerwein, Coen DA Stehouwer, Bert Bravenboer 


\section{Abstract}

\section{Aims}

The reported prevalence of type 2 diabetes mellitus (T2DM) in patients with liver cirrhosis is five times higher than in the general population. However, these data were never adjusted for classical risk factors for T2DM. We therefore investigated the association between cirrhosis and T2DM and adjusted for known risk factors for T2DM.

\section{Methods}

We reviewed medical files for presence of T2DM and potential confounders in 94 patients with cirrhosis (cases) and compared these to a control group of 107 patients with non-ulcer dyspepsia (NUD). Multiple logistic regression analysis was used to adjust for potential confounders.

\section{Results}

The aetiology of our cirrhosis population was alcohol (59\%), viral hepatitis (10\%), biliary cirrhosis (3\%) or cryptogenic (28\%). Prevalence of T2DM was significantly higher in patients with cirrhosis than in controls: $35 / 94$ (37\%) vs. $7 / 107$ (7\%) (OR 8.5, 95\%Cl 3.5-20.2, p<0.001). After adjustment for age, sex, family history of T2DM, alcohol use and BMI, cirrhosis remained significantly associated with T2DM (OR 13.6, 95\% $\mathrm{Cl} 4.3-42.9, \mathrm{p}<0.001)$. Most T2DM was already diagnosed before diagnosis of cirrhosis $(21 / 35,60 \%)$ or was incidentally found together with cirrhosis $(5 / 35$, $14 \%)$.

\section{Conclusions}

Liver cirrhosis had a strong, independent association with T2DM. Classical risk factors like family history and BMI could not explain the high T2DM prevalence in cirrhosis. Therefore, a liver-derived factor might aggravate glucose intolerance and cause T2DM in cirrhosis. In addition, T2DM might also cause cirrhosis through liver steatosis and fibrosis. 


\section{Introduction}

Abnormalities in glucose metabolism are common in patients with liver cirrhosis. The reported prevalence of type 2 diabetes mellitus (T2DM) in these patients ranges from $20-40 \%$, depending on the severity of liver disease and the diagnostic criteria used (1-8). This is about 5 times higher compared with the general age-matched population $(4,5)$. However, these data were not adjusted for classical risk factors for T2DM like family history of diabetes or body mass index (BMI), even though metabolic changes in liver disease are characterized by an insulin resistant state similar to patients with T2DM only $(1,9,10)$.

There are two proposed explanations for the high prevalence of diabetes in cirrhosis. Firstly, patients with cirrhosis may have a higher familial risk of diabetes or may be more obese. Secondly, the liver disease itself may cause alterations in glucose homeostasis (9). At this moment, it is still unclear whether family history and BMI contribute to the high prevalence of T2DM in liver cirrhosis $(3,8,11)$. The aims of this study were: firstly, to evaluate T2DM prevalence in patients with liver cirrhosis as compared with a control group of patients without liver disease. Secondly, to investigate the association between cirrhosis and T2DM with adjustment for potential confounding factors.

\section{Patients and methods}

\section{Patients}

We selected patients with liver cirrhosis by searching the digital diagnose-treatment combination registry for "compensated liver cirrhosis" and "decompensated liver cirrhosis". All patients $(n=202)$ treated in our hospital from January 2001 to September 2009 were found in this way. After careful review of the medical records, twenty-seven patients were excluded, because they did not have cirrhosis, but other liver diseases (Figure 5.2.1). Data on risk factors for T2DM were complete in 94 patients. Diagnosis of liver cirrhosis was established using liver biopsy or clinical signs together with biochemical abnormalities and evidence of cirrhosis on ultrasound or computed tomography. All patients underwent serological testing for hepatitis B and C virus. Cirrhosis was called cryptogenic, when an additional extensive evaluation provided no specific aetiology.

An age-matched control group was composed of patients with "non-ulcer dyspepsia" (NUD) in the same period (2001-2009). We chose this control group, since these patients are often extensively evaluated and have no increased risk for diabetes. We found 498 patients with diagnose-treatment combination "NUD", from which one age-matched control was randomly selected for each patient with cirrhosis. One control 
patient with a prolactinoma and three controls with liver disease were excluded, because T2DM is also associated with viral hepatitis and non-alcoholic hepatitis $(3,4,11-14)$. Data were complete in 107 controls.
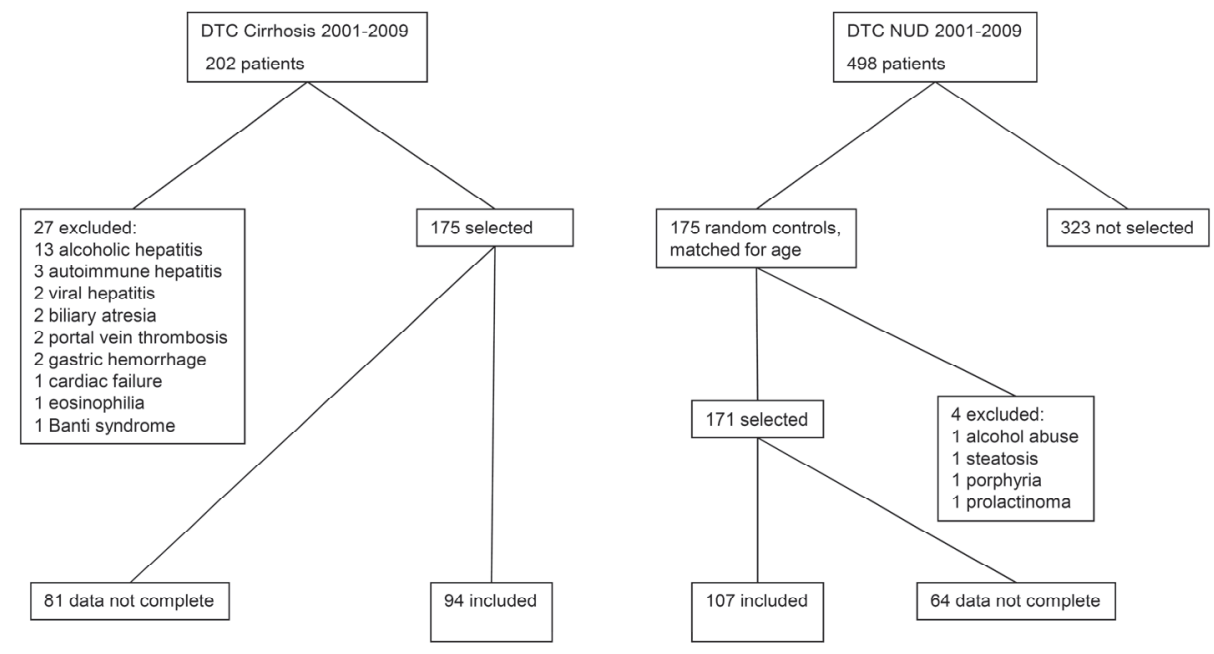

Figure 5.2.1 Flow chart of patient selection .

DTC: Diagnose-Treatment-Combination; NUD: Non-ulcer dyspepsia.

\section{Data collection}

Patient records were assembled from charts and laboratory measurements from the digital hospital information system retrospectively. Data collected at the time of diagnosis of liver cirrhosis or NUD were: age, sex, length, weight, family history of T2DM (first or second degree relatives), alcohol consumption, history of steroid drug use, history of T2DM, date of diagnosis of T2DM, aetiology of cirrhosis, serum alanine aminotransferase (ALAT), serum albumin, serum bilirubin, international normalized ratio (INR), serum creatinine, presence of ascites and presence of hepatic encephalopathy. BMI was calculated as weight (kilograms) divided by height in square meters $\left(\mathrm{kg} / \mathrm{m}^{2}\right)$. Alcohol use was classified as none, moderate (1-3 units daily) and excessive (more than 3 units daily). Severity of liver cirrhosis was classified using the Child-Pugh classification (15). We reviewed charts and laboratory measurements after diagnosis of cirrhosis or NUD for de novo T2DM. 


\section{Statistical analysis}

Baseline characteristics were compared using the Student's T-test or Mann-Whitney $\mathrm{U}$-test for continuous variables and $\chi 2$-test for discrete variables. We calculated the median duration between diagnosis of liver cirrhosis and diagnosis of T2DM. Multiple logistic regression analysis with forced entry was used to examine the association between cirrhosis and T2DM adjusting for potential confounders. Age and sex were always included in the regression model. Other determinants with $p$-values $\geq 0.20$ in univariate analysis were also entered in multivariate logistic regression. Testing for an interaction between sex and diagnosis (cirrhosis / NUD) was not significant ( $p$ for interaction $=0.887$ ). A two-sided $p$-value of $<0.05$ was considered statistically significant. All analyses were performed using the Statistical Package for Social Sciences (SPSS) for Windows, version 15.0 (SPSS Inc., Chicago, IL, USA).

\section{Results}

\section{Study population}

The sample population consisted of 94 patients with cirrhosis and 107 patients with NUD. Diagnosis of cirrhosis was confirmed by liver biopsy in $31 \%$. Patient characteristics did not differ between those with complete and incomplete data (data not shown). Table 5.2.1 shows baseline characteristics of patients and controls, and aetiology and Child-Pugh score in cirrhosis. Individuals with cirrhosis were more often male, had a higher alcohol consumption and slightly higher BMI. Serum ALAT, bilirubin and INR were higher and albumin was lower in the cirrhosis group than in the NUD group.

\section{Prevalence of diabetes mellitus}

T2DM was present in 35 patients with cirrhosis (37\%) and 7 controls with NUD (7\%). Diabetes had already been diagnosed in most patients before clinical onset of cirrhosis (21/35 patients, $60 \%)$ or was incidentally found together with the diagnosis cirrhosis (5/35, 14\%). Cirrhosis was diagnosed in patients with a history of T2DM after a median of 5.8 years (interquartile range 4.1-9.1). Nine of the remaining 68 patients without T2DM at diagnosis (13\%) developed de novo diabetes after a median of 1.1 years (interquartile range 0.8-2.3 years). The prevalence of T2DM was higher in cryptogenic cirrhosis $(54 \%)$ than in alcoholic cirrhosis $(31 \%)$ or viral cirrhosis $(40 \%)$. Patients with cryptogenic cirrhosis also had a higher BMI than patients with cirrhosis of other aetiology (30 kg/m² vs. $\left.26 \mathrm{~kg} / \mathrm{m}^{2} ; \mathrm{p}=0.009\right)$. 
Table 5.2.1 Baseline characteristics of patients with liver cirrhosis and non-ulcer dyspepsia controls.

\begin{tabular}{|c|c|c|c|}
\hline & Cirrhosis $(n=94)$ & NUD (n=107) & p-value \\
\hline Age (years) & $57 \pm 12$ & $59 \pm 13$ & 0.492 \\
\hline Sex (\% male) & 55 (59\%) & 42 (39\%) & 0.006 \\
\hline BMI $\left(\mathrm{kg} / \mathrm{m}^{2}\right)$ & $27 \pm 4.5$ & $26 \pm 4.4$ & 0.037 \\
\hline Total bilirubin $(\mu \mathrm{mol} / \mathrm{l})$ & $29(17-58)$ & $10(7-13)$ & $<0.001$ \\
\hline $\operatorname{ALAT}(U / I)$ & $38(27-65)$ & $23(17-31)$ & $<0.001$ \\
\hline Albumin (g/l) & $35 \pm 6.6$ & $44 \pm 3.3$ & $<0.001$ \\
\hline INR & $1.3(1.2-1.6)$ & $1.0(0.98-1.1)$ & $<0.001$ \\
\hline Creatinine $(\mu \mathrm{mol} / \mathrm{L})$ & $81(66-94)$ & $83(71-95)$ & 0.976 \\
\hline Positive family history of diabetes (\%) & $22(23)$ & $21(20)$ & 0.515 \\
\hline \multicolumn{4}{|l|}{ Alcohol consumption (\%) } \\
\hline None & $21(22)$ & $51(48)$ & $<0.001$ \\
\hline 1-3 daily & $17(18)$ & $52(48)$ & \\
\hline$>3$ daily & $56(60)$ & $4(4)$ & \\
\hline Use of steroids $n(\%)$ & $10(11)$ & $9(8)$ & 0.590 \\
\hline Diabetes mellitus & $35(37 \%)$ & $7(7 \%)$ & $<0.001$ \\
\hline \multicolumn{4}{|l|}{ Aetiology of cirrhosis (\%) } \\
\hline Alcoholic & 55 (59\%) & & \\
\hline Viral & $10(10 \%)$ & & \\
\hline Cryptogenic & $26(28 \%)$ & & \\
\hline Biliary & $3(3 \%)$ & & \\
\hline \multicolumn{4}{|l|}{ Child-Pugh score (\%) } \\
\hline A & $41(44 \%)$ & & \\
\hline B & $34(36 \%)$ & & \\
\hline $\mathrm{C}$ & $19(20 \%)$ & & \\
\hline
\end{tabular}

Results are expressed as mean \pm SD, frequencies (\%) or medians (inter-quartile range). ALAT: alanine aminotransferase; BMI: body mass index; INR: international normalized ratio; NUD: non-ulcer dyspepsia.

\section{Association of diabetes mellitus and liver cirrhosis}

In univariate logistic regression analysis, positive family history of diabetes (OR 2.6, $95 \% \mathrm{Cl} 1.2-5.5, \mathrm{p}=0.013$ ), moderate alcohol consumption (OR $0.29,95 \% \mathrm{Cl} 0.11-0.77$, $\mathrm{p}=0.013$ ), BMI (OR 1.2 per unit increase in $\mathrm{kg} / \mathrm{m}^{2}, 95 \% \mathrm{Cl} 1.1-1.3, \mathrm{p}<0.001$ ) and diagnosis of cirrhosis (OR $8.5,95 \% \mathrm{Cl} 3.5-20.2, \mathrm{p}<0.001$ ) were significantly associated with T2DM. After adjustment for age, sex, family history, alcohol use and BMI, cirrhosis remained significantly associated with T2DM (OR 13.6, 95\%Cl 4.3-42.9, p<0.001).

\section{Discussion}

This study showed that T2DM prevalence was five times higher in patients with cirrhosis than in an age-matched control group without liver diseases. This was not attributable to a higher familial risk or higher BMI in cirrhosis. In addition, the association remained strong after adjustment for potential confounders. 
These findings were in line with previous studies (3-5). The OR in the present study was higher than the one reported by Alavian et al. (i.e. adjusted OR, $6.5(95 \% \mathrm{Cl}$ 2.4-17.4) for viral cirrhosis using inactive hepatitis B carriers as controls (3). Only one other study compared patients with viral cirrhosis to a control group of subjects without liver disease (4). That study found T2DM in $23 \%$ and $5 \%$ of cases and controls respectively (OR 5.5, 95\% CI 3.5-8.5). These data were, however, not adjusted for family history and BMI.

The strong association between liver cirrhosis and T2DM does not mean that cirrhosis simply leads to T2DM, since $74 \%$ of diagnoses of T2DM were established before or at the moment liver cirrhosis was detected. Other prevalence studies also reported that $50-100 \%$ of T2DM had already been diagnosed before development of liver failure and cirrhosis $(2,3,6,16)$. Patients with viral and alcoholic hepatitis can have insulin resistance, IGT or T2DM $(3,4,11,12)$, likely caused by circulating pro-inflammatory cytokines $(12,17,18)$. Our study showed that T2DM was diagnosed no more than 10 years before occurrence of cirrhosis. Since both diseases may be detected after a subclinical period of 5-10 years after their onset, it is difficult to definitively establish the time course of the relationship (19-21).

In reverse, the high T2DM prevalence and high BMI in cryptogenic cirrhosis may imply that obesity leads to cirrhosis $(6,7,13,14)$. High levels of free fatty acids (FFA), resulting from inappropriate lipolysis, cause insulin resistance, but also liver steatosis (non alcoholic steatohepatitis) and cirrhosis (22-25). In addition, hyperglycaemia and hyperinsulinaemia may accelerate liver fibrosis and cirrhosis (26).

The strength of the present study is the comparison of patients with cirrhosis with a representative, age-matched control group (27). The main limitation of this study was its retrospective design, making analysis of patients and controls with complete data on risk factors for T2DM susceptible to selection bias. We believe that our sample was a representative selection, because patient characteristics and T2DM prevalence were not different between groups with complete and incomplete data. The incidence of de novo T2DM after diagnosis of cirrhosis in our study might be underestimated, since patients were not screened for T2DM once cirrhosis was diagnosed. Nevertheless, this does not influence the fact that, when cirrhosis was diagnosed, many patients already had overt T2DM. 


\section{References}

1. Megyesi C, Samols E, Marks V. Glucose tolerance and diabetes in chronic liver disease. Lancet 1967;2:1051-1056

2. Holstein A, Hinze S, Thiessen E, Plaschke A, Egberts EH. Clinical implications of hepatogenous diabetes in liver cirrhosis. J Gastroenterol Hepatol 2002;17:677-681

3. Alavian SM, Hajarizadeh B, Nematizadeh F, Larijani B. Prevalence and determinants of diabetes mellitus among Iranian patients with chronic liver disease. BMC Endocr Disord 2004;4:4

4. Custro N, Carroccio A, Ganci A, Scafidi V, Campagna P, Di Prima L, Montalto G. Glycemic homeostasis in chronic viral hepatitis and liver cirrhosis. Diabetes Metab 2001;27:476-481

5. Zein NN, Abdulkarim AS, Wiesner RH, Egan KS, Persing DH. Prevalence of diabetes mellitus in patients with end-stage liver cirrhosis due to hepatitis C, alcohol, or cholestatic disease. J Hepatol 2000;32: 209-217

6. Poonawala A, Nair SP, Thuluvath PJ. Prevalence of obesity and diabetes in patients with cryptogenic cirrhosis: a case-control study. Hepatology 2000;32:689-692

7. Tellez-Avila FI, Sanchez-Avila F, García-Saenz-de-Sicilia M, Chavez-Tapia NC, Franco-Guzman AM, LopezArce G, Cerda-Contreras E, Uribe M. Prevalence of metabolic syndrome, obesity and diabetes type 2 in cryptogenic cirrhosis. World J Gastroenterol 2008;14:4771-4775

8. Gentile S, Loguercio C, Marmo R, Carbone L, Del Vecchio Blanco C. Incidence of altered glucose tolerance in liver cirrhosis. Diabetes Res Clin Pract 1993;22:37-44

9. Petrides AS, Vogt C, Schulze-Berge D, Matthews D, Strohmeyer G. Pathogenesis of glucose intolerance and diabetes mellitus in cirrhosis. Hepatology 1994;19:616-27

10. Petrides AS, Groop LC, Riely CA, DeFronzo RA. Effect of physiologic hyperinsulinemia on glucose and lipid metabolism in cirrhosis. J Clin Invest 1991;88:561-570

11. Lecube A, Hernández C, Genescà J, Esteban Jl, Jardí R, Simó R. High prevalence of glucose abnormalities in patients with hepatitis $C$ virus infection. A multivariate analysis considering the liver injury. Diabetes Care 2004;27:1171-1175

12. Lecube A, Hernández C, Genescà J, Simó R. Glucose abnormalities in patients with hepatitis C virus infection. Epidemiology and pathogenesis. Diabetes Care 2006;29:1140-1149

13. Wong VWS, Hui AY, Tsang SWC, Chan JLY, Wong GLH, Chan AWH et al. Prevalence of undiagnosed diabetes and postchallenge hyperglycaemia in Chinese patients with non-alcoholic fatty liver disease. Aliment Pharmacol Ther 2006;24:1215-1222

14. Caldwell SH, Oelsner DH, lezzoni JC, Hespenheide EE, Battle EH, Driscoll CJ. Cryptogenic cirrhosis: clinical characterization and risk factors for underlying diease. Hepatology 1999;29:664-669

15. Pugh RNH, Murray-Lyon IM, Dawson JJ, Pietroni MC, Williams R. Transection of the oesophagus for bleeding oesophageal varices. Br J Surg 1973;60:646-649

16. Petit JM, Bour JB, Galland-Jos C, Minello A, Verges B, Guiguet M, Brun JM, Hillon P. Risk factors for diabetes mellitus and early insulin resistance in chronic hepatitis C. J Hepatol 2001;35:279-283

17. Lecube A, Hernández C, Genescà J, Simó R. Proinflammatory cytokines, insulin resistance, and insulin secretion in chronic hepatitis $C$ patients. A case-control study. Diabetes Care 2006;29:1096-1101

18. Tilg H, Diehl AM. Cytokines in alcoholic and nonalcoholic steatohepatitis. N Engl J Med 2000;343; 1467-1476

19. Harris MI, Klein R, Welborn TA, Knuiman MW. Onset of NIDDM occurs at least 4-7 yr before clinical diagnosis. Diabetes Care 1992;15:815-819

20. D'Amico G, Morabito A, Pagliaro L, Marubini E. Survival and prognostic indicators in compensated and decompensated cirrhosis. Dig Dis Sci 1986;31:468-475

21. Ginés $P$, Quintero $E$, Arroyo V, Terés J, Bruguera M, Rimola A, Caballería J, Rodés J, Rozman C. Compensated cirrhosis: natural history and prognostic factors. Hepatology 1987; 7:122-128

22. Hotamisligil GS, Arner P, Caro JF, Atkinson RL, Spiegelman BM. Increased adipose tissue expression of tumor necrosis factor-alpha in human obesity and insulin resistance. J Clin Invest 1995;95:2409-2415

23. lozzo P. Viewpoint on the way to the consensus session. Where does insulin resistance start? The adipose tissue. Diabetes Care 2009;32:S168-S173 
24. Liu S, Tinker L, Song Y, Rifai N, Bonds DE, Cook NR et al. A prospective study of inflammatory cytokines and diabetes mellitus in a multiethnic cohort of postmenopausal women. Arch Intern Med 2007;167:1676-1685

25. Tessari P, Coracina A, Cosma A, Tiengo A. Hepatic lipid metabolism and non-alcoholic fatty liver disease. Nutr Metab Cardiovasc Dis 2009;19:291-302

26. Picardi A, D'Avola D, Gentilucci UV, Galati G, Fiori E, Spataro S, Afeltra A. Diabetes in chronic liver disease: from old concepts to new evidence. Diabetes Metab Res Rev 2006;22:274-283

27. Baan CA, van Baal PH, Jacobs-van der Bruggen MA, Verkley H, Poos MJ, Hoogenveen RT, Schoemaker CG. Diabetes mellitus in the Netherlands: estimate of the current disease burden and prognosis for 2025. Ned Tijdschr Geneeskd 2009;153:1052-1058 


\section{Chapter}

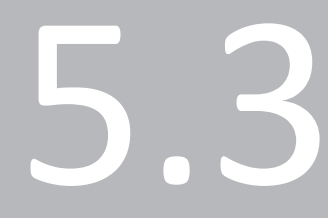

Diabetes mellitus at the time of diagnosis of cirrhosis is associated with higher incidence of spontaneous bacterial peritonitis, but not with increased mortality 


\section{Abstract}

Diabetes mellitus (DM) is present in $20-40 \%$ of patients with liver cirrhosis, but its prognostic impact is unclear. Therefore, we investigated whether the presence of DM in patients with cirrhosis was associated with increased mortality, and/or with an increased incidence of spontaneous bacterial peritonitis (SBP). We reviewed medical and laboratory data of 230 patients with cirrhosis from the period 2001-2011, of whom data were complete in $n=226$. Follow-up for the outcomes mortality and SBP was performed until May 2012, with only 13 patients lost to follow-up. DM was present at baseline in 78 patients (35\%). Median follow-up was 6.2 years [IQR 3.1-9.3], during which 118 patients died (47 out of 78 with DM (60\%), and 71 out of 148 without DM (48\%)). The presence of DM at baseline was not associated with increased mortality after adjustment for age (HR 1.00; 95\% Cl 0.67-1.50). Further adjustment for sex, aetiology of cirrhosis, platelet count, and the Child-Pugh or MELD score did not change this finding. During follow-up, 37 patients developed incident SBP (19 with DM and 18 without DM). DM at baseline was associated with incident SBP, even after adjustment for age, sex, aetiology, platelet count, and the Child-Pugh (HR 2.39; $95 \% \mathrm{Cl} 1.10-5.18$ ) or MELD score (HR 2.50; 95\% $\mathrm{Cl} 1.16-5.40$ ). In conclusion, the presence of DM at baseline in patients with cirrhosis was associated with an increased risk of SBP, which may represent an increased susceptibility to infections. On the other hand, DM was not clearly associated with increased mortality in these patients. 


\section{Introduction}

Diabetes mellitus (DM) is a common comorbidity in patients with liver cirrhosis. Several studies have reported a prevalence of $20-40 \%$ for DM in cirrhosis (1-5), depending on the aetiology and severity of cirrhosis, and the diagnostic criteria used. We have recently shown that cirrhosis itself was independently associated with DM (OR 13.6; $95 \% \mathrm{Cl} 4.3-42.9$ ) in a cross-sectional study, and that the increased prevalence of DM in cirrhosis was not attributable to classical risk factors for DM, such as age, obesity, or a family history of DM (3).

The prognostic impact of DM in patients with cirrhosis is currently unclear, although some studies have suggested that DM may be a risk factor for overall mortality. In the largest retrospective cohort study, with a median follow-up of 5 years, DM was associated with increased mortality (6), but not when the presence of oesophageal varices was included as covariate in a subgroup of 271 patients. Two small studies have also shown that DM was associated with increased 1-year (7) and 2-year mortality (8). In contrast, in two other prospective studies, DM did not influence the prognosis (1), or was no longer significantly associated with 2-year mortality after adjustment for Child-Pugh score and serum creatinine (9). These studies generally consisted of a small sample of patients $(n=<100)(1,7,8)$, had a low number of events $(n=<20)(1,7,9)$, and/or included patients with longstanding cirrhosis at baseline $(6,9)$.

The presence of DM may predispose to serious bacterial infections, as has been reported in patients with type 2 diabetes mellitus in general $(10,11)$. Moreover, patients with cirrhosis are characterized by immune dysfunction, which makes them highly susceptible to a variety of (bacterial) infections such as respiratory tract infections, urinary tract infections, sepsis, and spontaneous bacterial peritonitis (SBP) (12). Hence, bacterial infections were reported to be present at admission or developed during hospitalization in about $30 \%$ of patients with cirrhosis (13). Such infections are one of the most common causes of death in patients with cirrhosis, leading to a four-fold increased mortality (14). Currently, only a higher Child-Pugh score (or MELD score) is an established risk factor for infections $(12,15)$. Whether the presence of DM in patients with cirrhosis further increases the risk of infections, is as yet unclear, although the prevalence of bacteriuria was higher in patients with DM versus those without (16).

For these reasons, we investigated, in a relatively large cohort $(n>100)$, whether the presence of DM at diagnosis of cirrhosis was associated with increased overall mortality and liver-related mortality. In addition, we investigated whether DM was associated with incident spontaneous bacterial peritonitis (SBP), a common and serious infection in patients with cirrhosis $(13,17)$ that could be reliably retrieved and defined in our study. 


\section{Methods}

\section{Subjects and study design}

We conducted a retrospective observational study on a cohort of patients diagnosed with liver cirrhosis. Patients were selected by searching our digital diagnosis-treatment combination registry for codes "compensated liver cirrhosis" and "decompensated liver cirrhosis". We selected all patients treated for cirrhosis at our hospital from January 2001 till December 2011. The diagnosis of liver cirrhosis was based on liver biopsy, or on the combination of clinical signs, biochemical abnormalities, radiological findings (small, nodular liver, splenomegaly), and the presence of oesophageal varices or portal gastropathy on endoscopy. All patients were evaluated for the presence of Hepatitis $B$ and $C$ antibodies. In subjects without excessive alcohol consumption ( $\leq 2$ units/day for women, $\leq 3$ units/day for men), cirrhosis was called "cryptogenic" when an additional extensive evaluation yielded no specific aetiology. The study protocol was approved by the Medical Ethical Committee of the Catharina Hospital.

\section{Baseline data collection and definitions}

All data were collected from the medical files and the (digital) laboratory information system. The first day of admission to, or the first contact at the out-patient clinic of, our hospital was used as baseline. Data collected at this time were age, sex, current alcohol consumption, a history of diabetes mellitus, aetiology of cirrhosis, presence of ascites, and presence of hepatic encephalopathy. Alcohol consumption was categorized into low ( $<1$ unit/d), moderate (1-2 units/d for women, 1-3 units/d for men), and excessive alcohol consumption ( $>2$ units/day for women, and $>3$ units/day for men). Aetiology of cirrhosis was categorized as alcoholic, viral, autoimmune, and cryptogenic, with patients with cirrhosis after non-alcoholic steatohepatitis (NASH) grouped together with patients with cryptogenic cirrhosis, since several patients with cryptogenic cirrhosis may have had NASH before $(18,19)$. Patients with proven viral hepatitis and concomitant excessive alcohol consumption were classified as having viral cirrhosis. Standard laboratory methods were used to measure haemoglobin, platelet count (CELL-DYN 4000, Abbott), non-fasting blood glucose, aspartate aminotransferase (AST), alanine aminotransferase (ALT), alkaline phosphatase, gamma-glutamyl transferase (GGT), total bilirubin, albumin, creatinine (Siemens Advia 1650), and international normalized ratio (INR) (Roche STA) in all patients at the first contact.

The presence of DM at baseline, i.e. at the time of diagnosis of cirrhosis, was defined as a history of diagnosed DM (with or without medication), a random blood glucose $>11.1 \mathrm{mmol} / \mathrm{l}$ together with symptoms, or 2 fasting blood glucoses $>6.9 \mathrm{mmol} / \mathrm{l}$ (20). Because of frequent changes in therapy, we recorded the type of treatment most recently initiated for DM, categorized in no medical treatment, oral antihyperglycaemic agents, or insulin. The severity of liver cirrhosis was classified using the Child-Pugh 
score $(21,22)$, and the Model for End-stage Liver Disease (MELD) score based on bilirubin, creatinine, and INR $(22,23)$.

\section{Follow-up and mortality}

Follow-up of patients was performed until May 2012. We reviewed (digital) medical files of all specialities in our hospital from the diagnosis of cirrhosis until death or until the last visit to our hospital to any specialty. In the case of death, date and cause of death were retrieved from the medical files and death certificates wherever possible. Liver-related death was defined as death due to (a combination of) liver failure, hepatic encephalopathy, combined liver/kidney failure, sepsis, SBP, or hepatocellular carcinoma (HCC). In patients who were still alive in the previous year (May 2011 till May 2012) according to our records, the last visit to our hospital was taken as last date of follow-up. When patients had neither died, according to our records, nor visited our hospital in the previous year (May 2011 till May 2012), we contacted their general practitioners to inquire about their present status.

\section{Incident SBP and DM}

We reviewed all medical files, radiological examinations, and laboratory measurements for the development of the first SBP during follow up. Diagnostic paracentesis was performed in all patients with cirrhosis and ascites at hospital admission, and in patients with signs of systemic infection, abdominal symptoms, or acute kidney injury/liver failure. When ascitic polymorphonuclear (PMN) count was $\geq 250$ cells $/ \mathrm{mm}^{3}$, SBP was considered positive, and the patient was treated accordingly with iv antibiotics regardless of culture results, according to EASL guidelines (17). Patients with a PMN count $<250 / \mathrm{mm}^{3}$, but positive ascitic cultures, together with signs of systemic infection / inflammation and/or abdominal symptoms were also treated with iv antibiotics, in agreement with the EASL guidelines (17). Asymptomatic patients with positive ascitic cultures but without signs of infection or progressive renal/liver failure were considered SBP-free, were not treated with antibiotics, and were followed up clinically and/or by repeated paracentesis. The first date of admission for SBP was taken for (event-free) survival analyses. Because of high resistance of gut flora against quinolones (40\% of E. Coli quinolone-resistant) in our hospital / region, patients in our hospital did not receive primary antibiotic prophylaxis during the study period. In case patients experienced multiple episodes of SBP, the (time to the) first episode was taken for survival analyses. Recurrent SBP was also documented. Incident DM during follow-up at our hospital was defined using the definitions described above. 


\section{Statistical analysis}

General characteristics of the study population at baseline were compared between patients with and without DM at baseline using Student's independent t-test or Mann-Whitney $U$ test for continuous variables, and $\chi 2$-test for discrete variables.

Associations between baseline DM and the three outcomes, i.e. overall mortality, liver-related mortality, and incident SBP, were investigated with Kaplan-Meier curves and Log rank tests. In addition, Cox proportional hazard regression models were used to investigate the influence of potential confounders on the associations between DM and outcomes, with adjustment for age, sex, cirrhosis aetiology (alcoholic, cryptogenic, viral, auto-immune), platelet count, and severity of cirrhosis (continuous Child-Pugh or MELD score).

In an additional analysis, we investigated whether any of the main associations reported differed between sexes, severity of cirrhosis, and cirrhosis aetiology by adding interaction terms between DM and sex, Child-Pugh score, MELD score, or cirrhosis aetiology, respectively, to the (fully adjusted) models. A two-sided p-value of $<0.05$ was considered statistically significant. All analyses were performed using the Statistical Package for Social Sciences for Windows, version 17.0 (SPSS Inc., Chicago, IL, USA).

\section{Results}

\section{Study population and baseline characteristics}

We included 230 patients from the period 2001-2011, of whom data were complete in $\mathrm{n}=226$. Cirrhosis was proven by liver biopsy in $29 \%$ of patients. The aetiology of cirrhosis was mainly alcohol abuse (61\%), followed by cryptogenic / NASH cirrhosis (21\%), viral cirrhosis (11\%) and autoimmune liver diseases (4\%). In 8 patients (4\%), cirrhosis was due to other causes such as medication $(n=6)$, alpha 1-antitrypsin deficiency $(n=1)$, or hereditary haemochromatosis $(n=1)$. At inclusion in the present study, cirrhosis was newly diagnosed in 206 patients (91\%), and already known for 1-5 years in 20 patients.

Diabetes mellitus was present at baseline in 78 patients (35\%), of whom 17 were newly diagnosed. Sixteen patients $(21 \%)$ received no medical treatment, 19 (24\%) received oral antihyperglycaemic agents, and 43 were treated with insulin (55\%). Baseline characteristics of patients with and without DM are shown in Table 5.3.1. Patients with DM were older, and had a higher non-fasting glucose on admission compared with patients without DM. Aetiology of cirrhosis was more often NASH/cryptogenic in patients with DM, and predominantly alcoholic in patients without DM. In addition, patients with DM had generally less elevated liver enzymes compared with patients without DM, which could be attributed to the difference in alcohol (ab)use. However, the Child-Pugh and MELD score were not different between the two groups. 
Table 5.3.1 Baseline characteristics of the study population.

\begin{tabular}{|c|c|c|c|}
\hline & No DM (n=148) & DM $(n=78)$ & p-value \\
\hline Age (years) & $55.9 \pm 11.9$ & $65.4 \pm 10.9$ & $<0.001$ \\
\hline Male sex (\%) & 65 & 64 & 0.909 \\
\hline Alcohol consumption & & & $<0.001$ \\
\hline None (\%) & 17 & 44 & \\
\hline Moderate (\%) & 13 & 17 & \\
\hline Heavy (\%) & 70 & 40 & \\
\hline Biopsy proven (\%) & 23 & 33 & 0.225 \\
\hline Aetiology of cirrhosis & & & $<0.001$ \\
\hline Alcoholic (\%) & 70 & 44 & \\
\hline CC/NASH (\%) & 11 & 40 & \\
\hline Viral (\%) & 10 & 13 & \\
\hline Autoimmune (\%) & 5 & 3 & \\
\hline Other (\%) & 5 & 1 & \\
\hline Haemoglobin (mmol/l) & $7.49 \pm 1.53$ & $7.35 \pm 1.53$ & 0.516 \\
\hline Platelet count $\left(10^{9} / \mathrm{l}\right)$ & $150[86-217]$ & $140[95-181]$ & 0.308 \\
\hline Non-fasting glucose (mmol/l) & $6.3[5.6-7.3]$ & $9.8[7.1-12.8]$ & $<0.001$ \\
\hline AST (U/I) & 93 [47-163] & $56[37-88]$ & $<0.001$ \\
\hline $\operatorname{ALT}(U / I)$ & $41[29-66]$ & $39[32-51]$ & 0.344 \\
\hline Alkaline phosphatase (U/I) & 169 [106-248] & 120 [83-177] & $<0.001$ \\
\hline GGT (U/I) & 259 [98-548] & $140[87-290]$ & 0.008 \\
\hline Albumin (g/l) & $33.6 \pm 6.3$ & $34.1 \pm 6.3$ & 0.563 \\
\hline Total bilirubin $(\mu \mathrm{mol} / \mathrm{l})$ & $33[18-72]$ & $22[13-35]$ & $<0.001$ \\
\hline INR & $1.54 \pm 0.67$ & $1.48 \pm 0.43$ & 0.423 \\
\hline Creatinine $(\mu \mathrm{mol} / \mathrm{l})$ & $74[63-90]$ & 88 [71-107] & 0.001 \\
\hline MELD score & $12.2 \pm 7.5$ & $11.8 \pm 7.3$ & 0.681 \\
\hline Child-Pugh score & $7.8 \pm 2.1$ & $7.3 \pm 2.0$ & 0.099 \\
\hline Child-Pugh class & & & 0.238 \\
\hline$A(\%)$ & 32 & 41 & \\
\hline $\mathrm{B}(\%)$ & 43 & 42 & \\
\hline $\mathrm{C}(\%)$ & 25 & 17 & \\
\hline
\end{tabular}

Data are expressed as mean $\pm \mathrm{SD}$, median [interquartile range] or percentages. DM: diabetes mellitus; CC: cryptogenic cirrhosis; NASH: non-alcoholic steatohepatitis; AST: aspartate aminotransferase; ALT: alanine aminotransferase; GGT: gamma-glutamyl transferase; INR: international normalized ratio; MELD: model for end-stage liver disease.

\section{Mortality}

Follow-up was performed until May 2012, with 190 patients (84\%) followed solely at our hospital, $23(10 \%)$ followed by general practitioners in the end, and only 13 patients lost to follow-up (5.8\%; 2 with DM, 11 without DM). Median follow up was 6.2 years [IQR 3.1-9.3], during which 118 patients died (47 out of the 78 with DM (60\%), and 71 out of the 148 without DM (48\%)). Causes of death were classified as liver-related in 80 cases (Table 5.3.2). Median survival was 4.2 years $(95 \% \mathrm{Cl} 2.5-5.8)$ for patients with DM and 7.2 years $(95 \% \mathrm{Cl} 4.5-9.9)$ for patients without $\mathrm{DM}$ at baseline $(p=0.050)$. DM seemed to be associated with overall mortality in a crude analysis (HR $1.45 ; 95 \% \mathrm{Cl} 1.00$ 2.10), but this effect was completely attenuated after adjustment for age (HR 1.00; 
95\% Cl 0.67-1.50) (Table 5.3.3). Further adjustment for sex, aetiology, platelet count, Child-Pugh or MELD-score did not materially change this finding. In addition, DM was not associated with liver-related mortality in a crude analysis $(\mathrm{HR} 1.33 ; 95 \% \mathrm{Cl} 0.85$ 2.10), nor in fully adjusted models including Child-Pugh (HR $0.84 ; 95 \% \mathrm{Cl} 0.49-1.45$ ) or MELD score (HR 0.93; 95\% Cl 0.54-1.58).

Table 5.3.2 Causes of death.

\begin{tabular}{lccc}
\hline & No DM $(\mathrm{n}=148)$ & $\mathrm{DM}(\mathrm{n}=78)$ & Overall $(\mathrm{n}=226)$ \\
\hline Liver failure* $^{*}$ & 14 & 9 & 23 \\
Liver/kidney failure* $_{\text {Sepsis with/without liver failure* }}^{*}$ & 11 & 11 & 22 \\
SBP with/without liver failure* & 12 & 4 & 16 \\
(Variceal) bleeding* & 4 & 5 & 9 \\
Other infections & 8 & 2 & 10 \\
CVD & 4 & 3 & 7 \\
Cancer other than HCC & 3 & 1 & 4 \\
Unknown & 6 & 0 & 6 \\
Total & 9 & 12 & 21 \\
\hline
\end{tabular}

* classified as liver-related mortality. DM: diabetes mellitus; SBP: spontaneous bacterial peritonitis; CVD: cardiovascular diseases; HCC: hepatocellular carcinoma.

Table 5.3.3 Cox regression analysis of the effect of diabetes mellitus on mortality.

\begin{tabular}{lcccccc}
\hline & \multicolumn{3}{c}{ Overall mortality } & \multicolumn{3}{c}{ Liver-related mortality } \\
& HR & $95 \% \mathrm{Cl}$ & $\mathrm{p}$-value & HR & $95 \% \mathrm{Cl}$ & $\mathrm{p}$-value \\
\hline Crude & 1.45 & $1.00 ; 2.10$ & 0.051 & 1.33 & $0.85 ; 2.10$ & 0.215 \\
+ age & 1.00 & $0.67 ; 1.50$ & 0.999 & 0.97 & $0.59 ; 1.58$ & 0.897 \\
+ sex & 1.47 & $1.01 ; 2.14$ & 0.044 & 1.36 & $0.86 ; 2.15$ & 0.185 \\
+ aetiology & 1.47 & $1.00 ; 2.17$ & 0.053 & 1.29 & $0.80 ; 2.08$ & 0.300 \\
+ platelet count & 1.51 & $1.03 ; 2.21$ & 0.033 & 1.39 & $0.88 ; 2.21$ & 0.159 \\
+ Child-Pugh score & 1.46 & $1.00 ; 2.11$ & 0.048 & 1.39 & $0.88 ; 2.19$ & 0.157 \\
+ MELD score & 1.36 & $0.94 ; 1.97$ & 0.107 & 1.28 & $0.81 ; 2.02$ & 0.284 \\
& & & & & & \\
Fully adjusted (Child-Pugh)* & 0.90 & $0.58 ; 1.40$ & 0.638 & 0.84 & $0.49 ; 1.45$ & 0.539 \\
Fully adjusted (MELD)* & 0.96 & $0.62 ; 1.49$ & 0.871 & 0.93 & $0.54 ; 1.58$ & 0.778 \\
\hline
\end{tabular}

* Fully adjusted associations between diabetes mellitus and mortality were adjusted for age, sex, aetiology, platelet count, and the Child-Pugh score or MELD score, respectively.

\section{SBP}

During the follow-up period, 37 patients were admitted to our hospital with incident SBP (19 with DM and 18 without DM). SBP diagnosis was established on elevated ascitic PMN count in 26 cases (70\%), and based on positive cultures together with signs of systemic infection and/or acute liver/kidney failure in the remaining 11 patients (no differences between patients with or without $\mathrm{DM}, \mathrm{p}=0.800$ ). Altogether, a micro- 
biological agent was found in 22 cases (59\%) of SBP. The presence of DM was significantly associated with a higher incidence of SBP (Figure 5.3.1; $p=0.007$ ). DM was associated with incident SBP in a crude regression analysis ( $\mathrm{HR} 2.39 ; 95 \% \mathrm{Cl} 1.24-4.60$ ), and also in fully adjusted models including age, sex, aetiology, platelet count, and Child-Pugh (HR 2.39; $95 \% \mathrm{Cl} 1.10-5.18$ ) or MELD score (HR 2.50; 95\% Cl 1.16-5.40), respectively (Table 5.3.4). Of note, 22 episodes of SBP (59\%) were either on admission at the time cirrhosis was diagnosed $(n=9)$ or within 2 months of admission to our hospital $(n=13)$, as can be derived from Figure 5.3.1. On admission, 12 out of these 22 patients had DM, compared with 7 out of 15 patients that developed an SBP >2 months after the diagnosis of cirrhosis had been established $(P=0.638)$. As such, $D M$ at baseline was associated with a higher incidence of SBP both early after the diagnosis of cirrhosis had been established, and also more than 2 months after the diagnosis of cirrhosis.

Only three patients in our cohort (one with auto-immune cirrhosis (PSC), one with alcoholic cirrhosis, and one patient with cirrhosis due to hepatitis $C$ and alcohol) developed a second SBP (1 with DM and 2 without DM at baseline (and also without $\mathrm{DM}$ at follow-up), $\mathrm{P}=1.00$ ). Only the patient with alcoholic cirrhosis received secondary SBP prophylaxis after his first SBP (trimethoprim-sulfamethoxazole 960mg daily) for a few days, but soon discontinued this after resuming alcohol consumption.

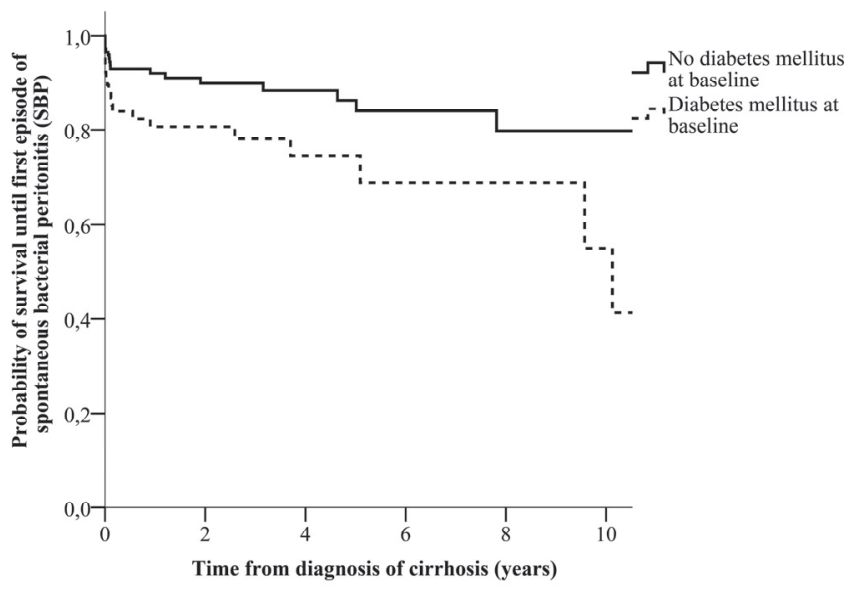

Figure 5.3.1 Incident spontaneous bacterial peritonitis in patients with cirrhosis according to the presence of diabetes mellitus at baseline (Logrank $\mathrm{P}=0.007$ ). 
Table 5.3.4 Cox regression analysis of the effect of diabetes mellitus on incident spontaneous bacterial peritonitis.

\begin{tabular}{lccc}
\hline & HR & $95 \% \mathrm{Cl}$ & p-value \\
\hline Crude & 2.39 & $1.24 ; 4.60$ & 0.009 \\
+ age & 2.43 & $1.16 ; 5.09$ & 0.019 \\
+ sex & 2.47 & $1.28 ; 4.78$ & 0.007 \\
+ aetiology & 2.81 & $1.40 ; 5.64$ & 0.004 \\
+ platelet count & 2.21 & $1.14 ; 4.25$ & 0.018 \\
+ Child-Pugh score & 2.55 & $1.32 ; 4.90$ & 0.005 \\
+ MELD score & 2.37 & $1.23 ; 4.56$ & 0.010 \\
& & & \\
Fully adjusted (Child-Pugh) & 2.39 & $1.10 ; 5.18$ & 0.027 \\
Fully adjusted (MELD) & 2.50 & $1.16 ; 5.40$ & 0.020 \\
\hline
\end{tabular}

* Fully adjusted associations between diabetes mellitus and spontaneous bacterial peritonitis were adjusted for age, sex, aetiology, platelet count, and the Child-Pugh score or MELD score, respectively.

\section{Incident DM}

During follow-up, 23 out of 148 patients (16\%) without DM at baseline developed de novo DM. The remaining 125 patients without DM at baseline were not structurally tested for DM, but at least had not developed clinical DM during follow-up at our hospital. The development of incident DM may attenuate, over time, the associations of DM at baseline with mortality and incident SBP. However, appreciation of (fully adjusted) Cox-regression models with a time-dependent variable (including an interaction term between diabetes at baseline and time) confirmed that the assumption of proportional hazards over time was not violated (all P-interaction $>0.10$ ), and that the prognostic effect of DM did not change over time. In addition, either censoring patients at the time of incident DM $(n=23)$, or confining analyses to subjects only followed up at our hospital ( $n=190)$ did not influence our results (data not shown).

In order to further investigate the effect of prevalent and incident DM on mortality and SBP, we conducted two analyses. First, we used a time-dependent Cox-regression model evaluating the effect of DM on mortality and SBP, with (clinical) DM as a time-dependent variable modelled at baseline and at 1, 3, and 5 years follow-up. In these models, the presence of DM (at baseline and/or follow-up) was also not associated with increased mortality (unadjusted $\mathrm{HR} 1.05,95 \% \mathrm{Cl} 0.62-1.76$; multivariate adjusted $\mathrm{HR} 0.76,95 \% \mathrm{Cl} 0.44-1.30$ ). In addition, DM (at baseline and/or follow-up) remained associated with incident SBP (unadjusted HR 2.26, 95\% Cl 1.05-4.90; multivariate adjusted HR 2.05, 95\% $\mathrm{Cl} 0.90-4.65)$. Second, we created 3 groups: patients with DM at cirrhosis diagnosis (i.e. DM at baseline), incident DM during follow-up, and the non-diabetic patients (who did not develop clinical DM; reference group). In Cox regression models using these groups, patients with DM at baseline had no increased mortality compared with those without DM at all (age-adjusted HR 0.88, $95 \% \mathrm{Cl} 0.57-1.36)$. Patients with incident DM also did not show an increased mortality 
(age-adjusted $\mathrm{HR} 0.64,95 \% \mathrm{Cl} 0.35-1.19)$. With regard to SBP, the risk of SBP was increased in patients with DM at baseline compared to those without DM at all (multivariate adjusted $\mathrm{HR} 2.43,95 \% \mathrm{Cl} 1.09-5.41$ ). Patients with incident DM did not show an increased risk of SBP (multivariate adjusted $\mathrm{HR} 0.86,95 \% \mathrm{Cl} 0.24-3.08$ ). In summary, the results of these analyses comparing the group with DM at baseline with no DM at all during follow-up were similar to those comparing DM at baseline with no DM at baseline (consisting of subjects that may or may not develop DM during followup).

\section{Additional analyses}

We also investigated whether the prognostic effect of DM in patients with cirrhosis differed between sexes, severity of cirrhosis, and cirrhosis aetiology. For (overall) mortality, we found no effect modification for sex, cirrhosis aetiology, or MELD score (all $\mathrm{P}$-interaction $>0.10)$. A borderline $\mathrm{P}$-value $(\mathrm{P}=0.078)$ for interaction between $\mathrm{DM}$ and the continuous Child-Pugh score suggested that the prognostic effect of DM might be stronger in those with lower Child-Pugh scores. However, no significant interaction was found for Child-Pugh classes (P-interaction=0.340). With regard to SBP, no effect modification was observed for sex, cirrhosis aetiology, continuous Child-Pugh score, or MELD score (all P-interaction $>0.10$ ).

As described above, the majority of patients (91\%) was newly diagnosed with cirrhosis at inclusion in the present study, while $n=20$ subjects were already known with cirrhosis for 1-5 years. Therefore, we also investigated potential differences between these groups and potential differences in the effect of DM on mortality and incident SBP. Patients with known cirrhosis $(n=206)$ and newly diagnosed cirrhosis $(n=20)$ had similar Child-Pugh score at baseline (7.2 \pm 2.2 vs $7.7 \pm 2.1 ; p=0.315)$, similar MELD score at baseline (11.6 \pm 7.2 vs $12.1 \pm 7.4 ; p=0.771)$, similar DM prevalence at baseline $(6 / 20$ vs. $72 / 206, p=0.657)$, similar mortality (11/20 known patients and 107/206 new patients; $\mathrm{p}=0.132)$, and similar SBP incidence (5/20 known patients and 32/206 new patients; $p=0.275$ ). In addition, the (absent) effect of DM at baseline on mortality was similar when patients with known cirrhosis $(n=20)$ were excluded from the regression analyses ( $\mathrm{HR}$ 1.05, 95\% $\mathrm{Cl} 0.66-1.67)$. Finally, the association between $\mathrm{DM}$ at baseline and incident SBP was comparable when patients with known cirrhosis $(n=20)$ were excluded from the regression analyses (unadjusted $\mathrm{HR} 2.10,95 \% \mathrm{Cl} 1.037-4.26$; multivariate $\mathrm{HR}$ $2.38,95 \% \mathrm{Cl} 1.04-5.44)$. In summary, we did not find different associations in patients with known vs. newly diagnosed cirrhosis. 


\section{Discussion}

The present study had two main findings. First, the presence of DM at the time of diagnosis of cirrhosis was not independently associated with an increased mortality. On the other hand, the presence of DM at the time of diagnosis of cirrhosis was significantly associated with an increased incidence of SBP, a serious infection for which hospitalization is usually required. This association was independent of known classical risk factors in patients with cirrhosis.

The crude borderline significant difference in survival we observed between patients with and without DM could be completely attributed to the fact that patients with DM were on average 10 years older than those without DM. Our results partially agree with the only other large retrospective cohort study, performed in 1980-1991, in which a small effect of DM on 5-year survival was observed (HR 1.18; 95\% Cl 1.03-1.37) independent of age, platelet count, and components of the Child-Pugh score (6). However, DM was, in that study, not associated with 3-year survival, nor with 5-year survival in the subgroup of 271 patients with endoscopic data. As in our study, patients with DM had similar Child-Pugh scores compared to those without DM, but were significantly older. In agreement, two small prospective studies also observed that DM was not (independently) associated with mortality $(1,9)$. Two studies showed that DM was associated with mortality independent of age $(7,8)$, but these studies were relatively small and contained a selected sample of patients with severe (alcoholic) cirrhosis and (refractory) ascites. Most patients with cirrhosis die because of liver-related complications such as liver failure, hepatorenal syndrome, variceal bleeding, hepatic encephalopathy, hepatocellular carcinoma, or SBP, as shown by our study and others $(1,6,8)$. The presence of hyperglycaemia and/or hyperinsulinaemia has been hypothesized to promote hepatitis and fibrosis, and accelerate the deterioration of liver function, hence leading to more liver-related deaths $(24,25)$. In agreement with this theory, the degree of insulin resistance has been correlated to the degree of fibrosis in patients with chronic hepatitis C (26), and the presence of DM was correlated with the degree of fibrosis in hereditary haemochromatosis (27). However, in patients with cirrhosis (i.e. grade 4 fibrosis), the presence of DM has not been associated with a worse liver function, as indicated by Child-Pugh score or MELD score $(6,7,9)$, nor with an increase in liver-related mortality in our study. As such, hyperglycaemia and/or hyperinsulinaemia may have a role in the progression of chronic liver diseases to cirrhosis, but apparently do not affect liver function when end-stage liver disease has been reached.

An important novel finding of the present study is that the incidence of SBP was about two-fold higher in patients with DM at baseline compared to those without DM. No previous studies have investigated this before, but a few case-control studies on subjects with ascites did show higher rates of DM in patients with SBP compared to those with sterile ascites, although not always significant due to small sample sizes 
$(15,28,29)$. The observed association between DM and SBP may represent an increased susceptibility to (bacterial) infections in subjects with both cirrhosis and DM, in agreement with observations that DM was also associated with more respiratory and urinary tract infections in patients with cirrhosis $(16,30)$. In addition, DM has recently been associated with SBP resistant to third-generation cephalosporins (31), which could reflect previous courses of antibiotics that have been given to these patients for other infections. Remarkably, the presence of DM among patients with SBP was not associated with an increased mortality in this study, in agreement with the similar liverrelated mortality (including SBP-related mortality) in patients with or without DM in our study. A potential explanation could be that patients with cirrhosis and DM are frequently monitored by gastroenterologists, internists, diabetes nurses, and/or general practitioners, leading to rapid recognition of incident infections and adequate treatment.

When confirmed by other studies, our results might imply that DM in patients with cirrhosis is a marker to identify subjects at increased risk for bacterial infections who might benefit from antibiotic prophylaxis, in addition to subjects with gastro-intestinal bleeding or ascites and advanced cirrhosis (32). The observation that most episodes of SBP occurred within 2 months of the diagnosis of cirrhosis might suggest that patients with cirrhosis that also have DM may be more prone to have or develop SBP, or infections in general, during admission, and should be monitored very closely. Antihyperglycaemic treatment may theoretically reduce the incidence of infections, but there have been no randomized controlled trials on treatment of DM in patients with cirrhosis. Only small observational studies suggest that good glycaemic control or treatment with metformin might be associated with decreased mortality $(33,34)$. It should be noted that medical treatment of DM in patients with cirrhosis is not without risks, as the combination of reduced hepatic clearance and impaired glycogenolysis may increase the risk of severe hypoglycaemia in patients on oral antihyperglycaemic medication or insulin (24). Therefore, the potential benefits and harms of treatment of DM in patients with cirrhosis should be carefully considered in each individual patient.

The present study consisted of a relatively large sample of patients with cirrhosis with a wide range of Child-Pugh / MELD scores. We obtained complete baseline data and a median follow-up of 6 years for most of them, and only 13 patients were lost to follow-up. A major limitation of the study is its retrospective design, which may lead to selection or information bias. However, all patients with cirrhosis must be coded with one of the used diagnosis-treatment combinations for financial purposes, and we have no reason to believe that patients with or without DM were differently registered. In addition, baseline data were structurally recorded for all patients as part of usual care, as were subsequent hospitalizations and mortality during follow-up. For these reasons, we feel that the retrospective design itself did not introduce considerable selection or information bias into the associations under investigation. However, we were unable to collect proper data on episodes of gastrointestinal bleeding, which is a risk factor 
for SBP (17,33). Although Child-Pugh score, MELD score, haemoglobin, and platelet counts were comparable between patients with and without DM, and all patients with gastrointestinal bleeding received antibiotic prophylaxis (7 days), we can therefore not fully exclude residual confounding. In addition, given our observations regarding SBP, it would be interesting to investigate whether DM is also associated with several other bacterial infections, and with other cirrhosis-related complications, such as ascites, hepatorenal syndrome (HRS), hepatic encephalopathy (HE), or hepatocellular carcinoma (HCC). However, the retrospective nature of our study precluded a prospective collection and proper definition of these complications (e.g. by regular creatinine measurement, or psychometric testing for $\mathrm{HE}$ ). Another potential limitation of our study is that we had no structural testing for incident DM, and we did not perform an oral glucose tolerance test (OGTT) at baseline to further classify normal glucose metabolism (NGM) or IGT. This potential misclassification could explain the fact that we did not observe differences in mortality between patients with and without DM. However, several additional analyses allowing for incident DM yielded similar findings, and other studies have not (yet) observed differences in mortality between patients with NGM, IGT and DM $(1,7)$.

In conclusion, we have shown that DM at the time of diagnosis of cirrhosis is an independent determinant of incident SBP in patients with cirrhosis. In agreement with other data on respiratory or urinary tract infections, the presence of DM may further increase the susceptibility for (bacterial) infections in patients with cirrhosis. On the other hand, this increased incidence of infections (in our case SBP) was not accompanied by an increased mortality in our study, although a prognostic effect of DM on mortality with $\mathrm{HR}<1.5$ cannot be excluded. Anyway, the increased rate of bacterial infections in patients with DM probably leads to more frequent hospitalizations and (antibiotic) treatments, with considerable health care costs. Therefore, it would be worthwhile to further investigate the role of DM in bacterial infections in patients with cirrhosis, and whether antihyperglycaemic treatment reduces the risk of bacterial infections, in particular those requiring hospitalization. 


\section{References}

1. Holstein A, Hinze S, Thiessen E, Plaschke A, Egberts EH: Clinical implications of hepatogenous diabetes in liver cirrhosis. J Gastroenterol Hepatol 2002;17:677-681

2. Alavian SM, Hajarizadeh B, Nematizadeh F, Larijani B: Prevalence and determinants of diabetes mellitus among Iranian patients with chronic liver disease. BMC Endocr Disord 2004;4:4

3. Wlazlo N, Beijers HJ, Schoon EJ, Sauerwein HP, Stehouwer CD, Bravenboer B: High prevalence of diabetes mellitus in patients with liver cirrhosis. Diabet Med 2010;27:1308-1311

4. Zein NN, Abdulkarim AS, Wiesner RH, Egan KS, Persing DH: Prevalence of diabetes mellitus in patients with end-stage liver cirrhosis due to hepatitis C, alcohol, or cholestatic disease. J Hepatol 2000;32: 209-217

5. Gentile S, Loguercio C, Marmo R, Carbone L, Del Vecchio Blanco C: Incidence of altered glucose tolerance in liver cirrhosis. Diabetes Res Clin Pract 1993;22:37-44

6. Bianchi G, Marchesini G, Zoli M, Bugianesi E, Fabbri A, Pisi E: Prognostic significance of diabetes in patients with cirrhosis. Hepatology 1994;20:119-125

7. Hagel S, Bruns T, Herrmann A, Stallmach A, Schmidt C: Abnormal glucose tolerance: a predictor of 30day mortality in patients with decompensated liver cirrhosis. Z Gastroenterol 2011;49:331-334

8. Moreau R, Delegue P, Pessione F, Hillaire S, Durand F, Lebrec D, Valla DC: Clinical characteristics and outcome of patients with cirrhosis and refractory ascites. Liver Int 2004;24:457-464

9. Quintana JO, Garcia-Compean D, Gonzalez JA, Perez JZ, Gonzalez FJ, Espinosa LE, Hernandez PL, Cabello ER, Villarreal ER, Rendon RF, Garza HM: The impact of diabetes mellitus in mortality of patients with compensated liver cirrhosis-a prospective study. Ann Hepatol 2011;10:56-62

10. Geerlings SE, Hoepelman Al: Immune dysfunction in patients with diabetes mellitus (DM). FEMS Immunol Med Microbiol 1999;26:259-265

11. Rajagopalan S: Serious infections in elderly patients with diabetes mellitus. Clin Infect Dis 2005;40: 990-996

12. Christou L, Pappas G, Falagas ME: Bacterial infection-related morbidity and mortality in cirrhosis. Am J Gastroenterol 2007;102:1510-1517

13. Fernandez J, Navasa M, Gomez J, Colmenero J, Vila J, Arroyo V, Rodes J: Bacterial infections in cirrhosis: epidemiological changes with invasive procedures and norfloxacin prophylaxis. Hepatology 2002;35:140-148

14. Arvaniti V, D'Amico G, Fede G, Manousou P, Tsochatzis E, Pleguezuelo M, Burroughs AK: Infections in patients with cirrhosis increase mortality four-fold and should be used in determining prognosis. Gastroenterology 2010;139:1246-1256, 1256 e1241-1245

15. Obstein KL, Campbell MS, Reddy KR, Yang YX: Association between model for end-stage liver disease and spontaneous bacterial peritonitis. Am J Gastroenterol 2007;102:2732-2736

16. Amato A, Precone DF, Carannante N, Brancaccio G, Stornaiuolo G, Galante D, Gaeta GB: [Prevalence and risk factors for bacteriuria in patients with cirrhosis]. Infez Med 2005;13:103-108

17. European Association for the study of the Liver, EASL clinical practice guidelines on the management of ascites, spontaneous bacterial peritonitis, and hepatorenal syndrome in cirrhosis. J Hepatol 2010;53: 397-417

18. Farrell GC, Larter CZ: Nonalcoholic fatty liver disease: from steatosis to cirrhosis. Hepatology 2006; 43:S99-S112

19. Caldwell SH, Oelsner DH, lezzoni JC, Hespenheide EE, Battle EH, Driscoll CJ: Cryptogenic cirrhosis: clinical characterization and risk factors for underlying disease. Hepatology 1999;29:664-669

20. WHO: Definition, diagnosis and classification of diabetes mellitus. Report of a WHO Consultation. Part 1: Diagnosis and classification of diabetes mellitus. . In WHO/NCD/NCS/992 Geneva, Switzerland, 1999

21. Pugh RN, Murray-Lyon IM, Dawson JL, Pietroni MC, Williams R: Transection of the oesophagus for bleeding oesophageal varices. Br J Surg 1973;60:646-649

22. Durand F, Valla D: Assessment of the prognosis of cirrhosis: Child-Pugh versus MELD. J Hepatol 2005;42 Suppl:S100-107 
23. Kamath PS, Wiesner RH, Malinchoc M, Kremers W, Therneau TM, Kosberg CL, D'Amico G, Dickson ER, Kim WR: A model to predict survival in patients with end-stage liver disease. Hepatology 2001;33: 464-470

24. Wlazlo N, Sauerwein HP, Schoon EJ, Stehouwer CD, Bravenboer B: [Diabetes mellitus and cirrhosis of the liver: a prognostically unfavourable combination]. Ned Tijdschr Geneeskd 2011;155:212-219; A2213

25. Garcia-Compean D, Jaquez-Quintana JO, Gonzalez-Gonzalez JA, Maldonado-Garza H: Liver cirrhosis and diabetes: risk factors, pathophysiology, clinical implications and management. World J Gastroenterol 2009;15:280-288

26. Hui JM, Sud A, Farrell GC, Bandara P, Byth K, Kench JG, McCaughan GW, George J: Insulin resistance is associated with chronic hepatitis $\mathrm{C}$ virus infection and fibrosis progression [corrected]. Gastroenterology 2003;125:1695-1704

27. Wood MJ, Powell LW, Dixon JL, Ramm GA: Clinical cofactors and hepatic fibrosis in hereditary hemochromatosis: The role of diabetes mellitus. Hepatology 2012;56:904-911

28. Bajaj JS, Zadvornova Y, Heuman DM, Hafeezullah M, Hoffmann RG, Sanyal AJ, Saeian K: Association of proton pump inhibitor therapy with spontaneous bacterial peritonitis in cirrhotic patients with ascites. Am J Gastroenterol 2009;104:1130-1134

29. Sorrentino P, Tarantino G, Conca P, Perrella A, Perrella O: Clinical presentation and prevalence of spontaneous bacterial peritonitis in patients with cryptogenic cirrhosis and features of metabolic syndrome. Can J Gastroenterol 2004;18:381-386

30. Diaz J, Monge E, Roman R, Ulloa V: Diabetes as a risk factor for infections in cirrhosis. Am J Gastroenterol 2008;103:248

31. Ariza X, Castellote J, Lora-Tamayo J, Girbau A, Salord S, Rota R, Ariza J, Xiol X: Risk factors for resistance to ceftriaxone and its impact on mortality in community, healthcare and nosocomial spontaneous bacterial peritonitis. J Hepatol 2012;56:825-832

32. Fernandez J, Gustot T: Management of bacterial infections in cirrhosis. J Hepatol 2012;56 Suppl 1:S1-12

33. Nkontchou G, Cosson E, Aout M, Mahmoudi A, Bourcier V, Charif I, Ganne-Carrie N, Grando-Lemaire V, Vicaut $\mathrm{E}$, Trinchet JC, Beaugrand M: Impact of metformin on the prognosis of cirrhosis induced by viral hepatitis C in diabetic patients. J Clin Endocrinol Metab 2011;96:2601-2608

34. Kwon SY, Kim SS, Kwon OS, Kwon KA, Chung MG, Park DK, Kim YS, Koo YS, Kim YK, Choi DJ, Kim JH: Prognostic significance of glycaemic control in patients with HBV and HCV-related cirrhosis and diabetes mellitus. Diabet Med 2005;22:1530-1535 


\section{Chapter 6}

Summary and discussion 
Chapter 6 


\section{General discussion}

The central objective of this thesis was to study to the aetiology of insulin resistance and accompanying metabolic complications, such as disorders in glucose and lipid metabolism, and fatty liver disease, in humans. Specifically, we focussed on the role of three novel factors and/or mechanisms: iron metabolism, the complement system, and liver cirrhosis. In this final chapter we summarize the main findings of this thesis, address some methodological issues related to the epidemiological studies conducted, and discuss potential implications and directions for future research.

\section{Main findings}

\section{Iron metabolism}

In Chapter 3, we investigated the role of iron metabolism in insulin resistance (IR) and glucose intolerance in two epidemiological evaluations of the Cohort on Diabetes and Atherosclerosis Maastricht (CODAM) study. In Chapter 3.2, we showed that four markers of iron-metabolism (serum ferritin, transferrin, total iron, and non-transferrin-bound iron (NTBI)) were cross-sectionally associated with a novel index of adipocyte IR in humans. These findings were corroborated by positive associations of these markers with plasma non-esterified fatty acids (NEFAs), and by inverse associations of serum ferritin and transferrin with adiponectin, a specific marker of adipose tissue dysfunction $(1,2)$. Finally, we observed that total antioxidant status (TAS) acted as a significant mediator in the associations of ferritin and transferrin with adipocyte IR and HOMA2-IR, which supports the hypothesis that the mechanism between iron and insulin resistance may involve induction of oxidative stress.

In Chapter 3.3, we showed that, over a 7-year period, serum ferritin and transferrin at baseline were prospectively associated with HOMA2-IR, adipocyte IR, and a novel marker of hepatic IR, independently of classical risk factors and inflammatory markers. In addition, serum ferritin and transferrin were longitudinally associated with glucose tolerance, including $A \cup C_{\text {glucose }}$ during the OGTT. Lastly, we observed similar positive associations for serum iron and NTBI, although not statistically significant.

Altogether, our findings from Chapter $\mathbf{3 . 2}$ and $\mathbf{3 . 3}$ suggest that body iron and/or iron-metabolism-related factors may be involved in the development of insulin resistance in several tissues, including muscle, liver, and adipocytes, eventually leading to impaired glucose metabolism and T2DM.

\section{The complement system}

In Chapter 4, we examined the complement system in relation to obesity, insulin resistance, T2DM, and fatty liver disease in four studies within CODAM. In Chapter 4.2, 
we cross-sectionally explored metabolic pathways that underlie the obesity-related differences in circulating C3 levels. Using multiple mediation analyses, we observed that the association between obesity and circulating C3 could be explained for $20-25 \%$ by fat-related low-grade inflammation and for $40-55 \%$ by fat-related insulin resistance, independently of each other. In Chapter 4.3, we observed that baseline C3 levels were associated with incident T2DM during 7-year follow-up, independent of obesity. In addition, (changes in) C3 levels were strongly associated with (changes in) the degree of insulin resistance in muscle, liver and adipocytes. The results from these two studies thus suggest that changes in plasma C3 levels reflect a progression of metabolic dysregulation, including progression of low-grade inflammation and insulin resistance, that eventually leads to abnormalities in glucose tolerance and frank T2DM.

In Chapters 4.4 and 4.5, we investigated the role of complement activation in human alcoholic and non-alcoholic fatty liver disease (AFLD and NAFLD) in two cross-sectional studies. First, we showed that plasma C3a levels, as a marker of complement activation via any of the three pathways, were associated with (estimated) liver fat and hepatocellular injury, particularly in individuals with heavy alcohol consumption (Chapter 4.4). A significant portion (30\%) of these associations was mediated by the pro-inflammatory cytokine tumour necrosis factor alpha (TNF- $\alpha$ ). In the second study (Chapter 4.5), we showed that plasma levels of sC5b-C9, as a marker of terminal pathway activation, were consistently associated with (estimated) liver fat and hepatocellular injury, both in no-to-moderate and heavy alcohol consumers. Contrary to the associations of $\mathrm{C} 3 \mathrm{a}$, these associations were not mediated by TNF- $\alpha$. Taken together, these findings suggests that complement activation may contribute to the spectrum of AFLD and NAFLD in humans, both through anaphylotoxin signalling leading to TNF- $\alpha$ production in Kupffer cells, and through terminal complement activation.

\section{Liver cirrhosis}

In Chapter 5, we examined the relation between T2DM and liver cirrhosis in two studies performed within a retrospective cohort of patients with liver cirrhosis. In Chapter 5.2, we showed that the prevalence of T2DM was about five times higher in patients with cirrhosis (37\%) compared to (healthy) controls with non-ulcer dyspepsia (7\%). We also showed that this high prevalence was independent of classical risk factors for T2DM, such as age, obesity, and a positive family history of T2DM. In Chapter 5.3, we investigated the prognostic impact of this high prevalence of T2DM on the course of liver cirrhosis. We observed that the presence of T2DM at the time of diagnosis of cirrhosis was not independently associated with an increased (all-cause and liverrelated) mortality, although a prognostic effect with hazard ratio below 1.5 could not be fully excluded. On the other hand, T2DM was significantly associated with a two-fold increased risk of spontaneous bacterial peritonitis (SBP), a serious infection for which hospitalization is usually required. 


\section{Methodological considerations}

\section{Study population and designs - external validity}

Most studies in this thesis (Chapters $\mathbf{3}$ and $\mathbf{4}$ ) were conducted within the CODAM study, which consists of a selected group of middle-aged and elderly Caucasian individuals. It is uncertain whether the results obtained in these individuals can be extrapolated to other ethnicities or younger individuals, and additional studies are necessary to confirm our findings in these populations. In addition, participants of the CODAM study were specifically selected for an increased risk for metabolic and cardiovascular diseases (CVD) (Chapter 2.1), and therefore represent a relatively unhealthy subgroup of the general population. For example, the prevalence of overweight and obesity, at baseline, was $51 \%$ and $31 \%$ respectively in the CODAM study, compared with $36 \%$ and $13 \%$ in the general population in the Netherlands (3). In addition, the prevalence of T2DM and IGM was $25 \%$ and $22 \%$ respectively in the CODAM study, compared with the lower prevalence in the general Dutch population (5-7\% for T2DM, 6-8\% for IGM) (4). Generalizability of our data is therefore restricted to other populations with a similar high risk profile. However, such issues around the representativeness of the study population probably do not affect the pathophysiological validity of our findings. In addition, the CODAM study population represents a large group of individuals who are present in the (Western) general population, in whom cardiovascular and metabolic diseases are most likely to develop over time. As such, the data presented in this thesis can be generalized to this large group of high-risk individuals, and increase our knowledge on the mechanisms that lead to the development of metabolic diseases, such as T2DM and fatty liver disease.

Two studies were performed in a Dutch cohort of patients with liver cirrhosis (Chapter 5). Our study population consisted of an unselected, diverse group of patients with cirrhosis, representing all Child-Pugh classes. As such, the findings presented in this thesis regarding cirrhosis and T2DM may well be generalized to other populations of patients with liver cirrhosis throughout the (Western) world. However, as our population consisted mainly of individuals with alcoholic and cryptogenic / nonalcoholic steatohepatitis cirrhosis, and less viral and auto-immune cirrhosis, extrapolation of our data to these groups should be done with caution, and other studies should confirm our findings in these subgroups.

\section{Study population and designs - internal validity}

In observational research, several potential sources of bias may obscure true associations and thus impede interpretation of the obtained results. Among these are selection bias, information bias, and confounding (5). 


\section{Selection bias}

In observational studies, an association between two factors may be obscured by selection bias when individuals are selected on a mutual consequence of these two factors $(6,7)$. For the cross-sectional studies performed within CODAM, such selection bias could have been introduced if -for instance- only individuals with T2DM would have been selected. Restriction of analyses to patients with T2DM only would have induced a downward bias in the association between two intermediate factors, e.g. serum ferritin and HOMA2-IR, or C3 levels and HOMA2-IR (Figure 6.1A). However, although the CODAM study population consisted of selected individuals with an increased risk for T2DM based on a combination of risk factors, there was no complete conditioning on outcomes such as T2DM. Hence the results obtained in the crosssectional studies here were likely not affected by selection bias.

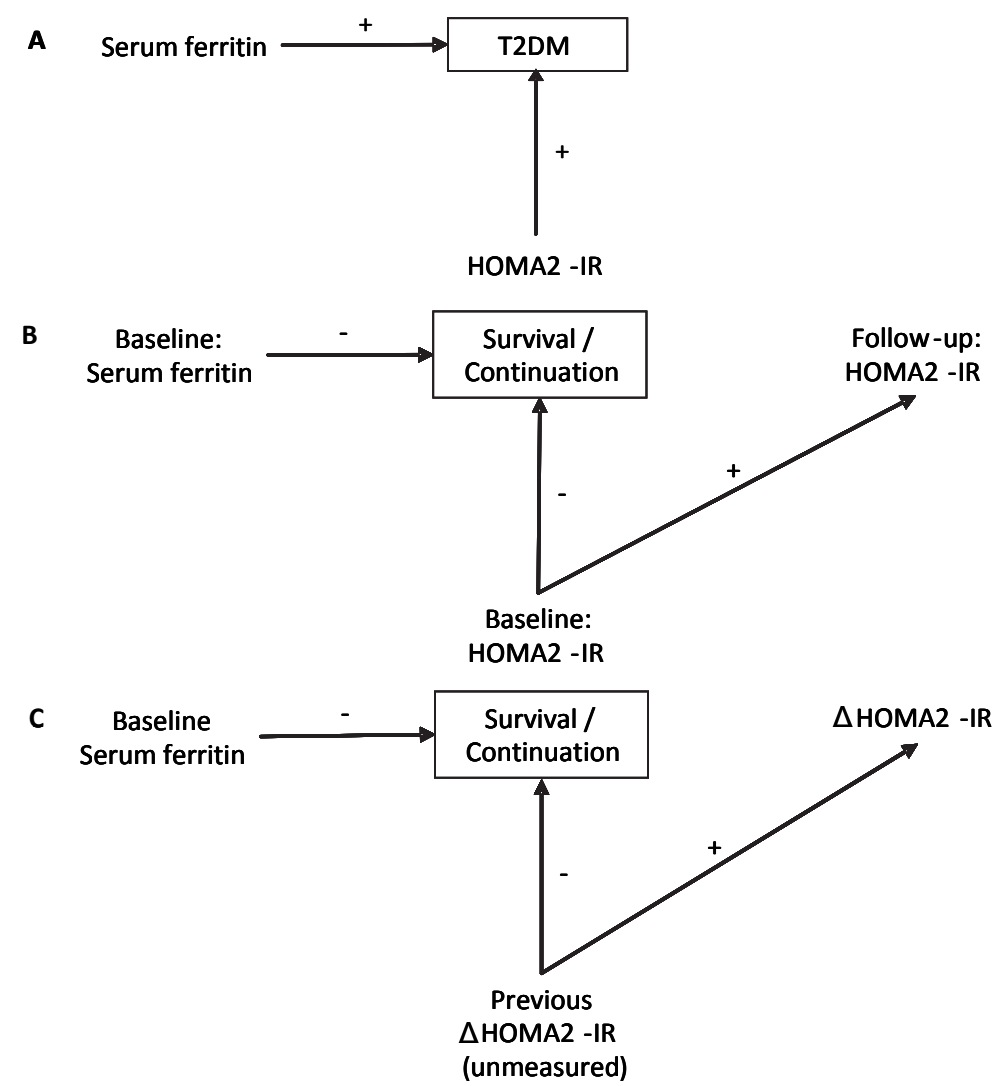


Figure 6.1 Directed acyclic graphs (DAGs) illustrating potential sources of selection bias in studies performed with CODAM data. Panel A shows two determinants of T2DM that are not associated with each other under the null hypothesis (e.g. ferritin and HOMA2-IR). However, within the group of individuals with T2DM, an inverse association between ferritin and HOMA2-IR may be observed (i.e. individuals that developed T2DM 'due to' high ferritin probably have a low HOMA2-IR), since conditioning on a collider (in this case T2DM) may induce a statistical association between the two proximal determinants $(6,7)$. As such, restricting analyses to individuals with T2DM only induces a downward bias in the association between ferritin and HOMA2-IR, resulting in an underestimation of effect sizes. Panel B shows the potential for selection bias ( attrition bias) in longitudinal studies, when analyses are restricted to individuals that attend follow-up. Both the determinants of interest (e.g. ferritin) and the outcome (e.g. HOMA2-IR) may negatively influence survival or continuation in the CODAM study, as indicated by the negative signs. In addition, HOMA2-IR at baseline is positively associated with HOMA2-IR at follow-up. Within the group of individuals that remain in the study during follow-up, an inverse association may be observed between ferritin and HOMA2-IR at baseline, as the remaining individuals with high ferritin levels will have had lower HOMA2-IR at baseline $(6,8)$. As such, restricting analyses to individuals who continued the study represents a form of conditioning on a collider, and may induce a downward bias in the associations between ferritin with HOMA2-IR at baseline and at follow-up (i.e. GEE associations in Chapter 3.3). Panel $C$ shows another example of potential attrition bias in longitudinal studies, but now regarding the analysis of changes. Again, the determinants of interest are hypothesized to negatively influence survival. The outcome of interest is now defined as changes in HOMA2-IR during the study period, and these changes are probably preceded by (and correlated with) changes in HOMA2-IR before the start of the study period, caused by a variety of factors not specified here. These previous changes in HOMA2-IR may be negatively associated with survival and study continuation. Analogous to panel B, restricting analyses to individuals who continued the study represents conditioning on a collider, and would, if the above-described relations were actually present, induce a downward bias on associations between ferritin and changes in HOMA2-IR (Chapter 3.3).

In longitudinal studies, selection bias often results from selective drop-out of the unhealthiest participants (8). In CODAM, $14 \%$ of individuals participating in the baseline measurement did not attend follow-up, and these individuals were generally unhealthier at baseline. As such, selective attrition may introduce bias into associations between the determinants under study in the present thesis and (changes in) metabolic factors (Figure 6.1B and $1 \mathrm{C}$ ), depending on the extent to which these determinants are also related to attrition. We consider the risk of attrition bias in our studies quite low for the following reasons. Firstly, in the longitudinal study presented in Chapter 4.3, individuals not attending follow-up did not differ in circulating C3 levels. Secondly, although, in Chapter 3.3, individuals not attending follow-up had somewhat higher ferritin levels compared with individuals attending follow-up (although not statistically significant), they did not differ in other markers of iron metabolism. For analyses concerning ferritin, or perhaps iron metabolism in general, restricting analyses to individuals with follow-up data available might therefore have introduced a downward bias into the associations, resulting in an underestimation of effect sizes. This could have accounted for the fact that we did not observe significant associations between markers of iron metabolism at baseline and changes in insulin resistance variables. 
However, to reduce the influence of selection bias due to attrition as efficiently as possible, we performed GEE analyses including data from all individuals as main analysis in these analyses.

The results obtained in Chapter $\mathbf{5}$ were also not likely affected by selection bias as all patients with liver cirrhosis attending the Catharina hospital were coded with one of the diagnosis-treatment combinations for financial purposes, and we have no reason to believe that patients with or without T2DM were differently registered (hence leading to selection). Conversely, the use of patients with non-ulcer dyspepsia (NUD) as controls in Chapter $\mathbf{5 . 2}$ might to some extent have introduced selection bias, as patients with T2DM visiting outpatient clinics of internal medicine may be referred more quickly to a gastroenterologist than healthy individuals in the general population. Such a selection bias would have led to a relatively high prevalence of T2DM in patients with NUD, and hence an underestimation of the true association between cirrhosis and T2DM. However, as the prevalence of T2DM in the control group of individuals with NUD was $7 \%$ and comparable to the prevalence of T2DM in the general population (9), we are confident that our analyses in Chapter $\mathbf{5 . 2}$ have not been influenced by selection bias. Finally, in Chapter 5.3, there was no conditioning on survival or continuation, as survival was the primary outcome, analysed using Cox proportional hazard models to deal with drop-out.

\section{Information bias}

Errors in the measurement of variables may lead to misclassification of individuals, and hence biased data and associations. Such misclassification can be either differential or non-differential. Differential misclassification occurs when the measurement error differs across groups of the determinant under study, and may cause either under- or overestimation of true associations (10). In the present thesis, however, almost all the variables of interest were biomarkers (e.g. markers of iron metabolism, complement factors, glucose, insulin, fatty acids, etc.) that were determined in blood samples of study participants. Each was measured in the same way, by assessors that were blinded with respect to any of the outcome variables. The only potential source of differential misclassification may lie in the relative high amount of haemolysis in the follow-up visit of the CODAM study ( $9 \%$ light haemolysis, 13\% strong haemolysis) compared with little to no haemolysis in the baseline visit ( $1 \%$ and $0.5 \%$ respectively). In individuals with overweight or obesity, venepuncture may be more difficult, leading to more haemolysis, and hence falsely elevated levels of liver enzymes and insulin at the followup visit. However, we did not observe an association between measures of obesity and the amount of haemolysis in this visit (data not shown). As such, the higher amount of haemolysis in the follow-up visit will probably not have introduced differential misclassification, but only to non-differential misclassification.

Non-differential misclassification occurs when the measurement error is equal across groups of the determinant under study (i.e. random error), and generally 
leads to an underestimation of true associations and reduced power (see also Chapter 2.5) (11). Both analytical measurement error (i.e. the precision of the measurement) and biological variability (i.e. real but temporary deviations from an individual's usual value) contribute to this random error. As the studies in the present thesis mainly make use of biomarkers measured in blood, such non-differential misclassification may indeed have led to underestimated effect sizes in the associations reported here. In addition, we used these biomarkers, individually or combined into a score or formula, to estimate insulin resistance (whole-body and in specific tissues), glucose tolerance, hepatic steatosis, and hepatocellular injury (see Chapter 2.3). We did not perform (more invasive) gold standard techniques such as hyperinsulinaemic euglycaemic clamp studies, liver or fat biopsies, or proton magnetic resonance spectroscopy $\left({ }^{1} \mathrm{H}-\mathrm{MRS}\right)(12-15)$, as this was not achievable within our cohort study with 574 participants. However, as the outcome variables used correlate well with these gold standards (Chapter 2.3), this only implies that the true effect sizes are at least equal or even larger than the ones observed here.

A major limitation of the studies in Chapter $\mathbf{5}$ is the retrospective design, which may lead to information bias, especially when determinants are measured retrospectively. This was, however, not the case in our studies, since all baseline data (again, mostly biomarkers) were structurally recorded in the (digital) hospital information system for all patients as part of usual care in the study period, as were subsequent hospitalizations and mortality during follow-up. In addition, we tried to prevent differential misclassification of death and survival as much as possible by contacting general practitioners of patients that did not attend the outpatient clinic anymore, yielding a complete follow-up in $94 \%$ of patients with no difference in individuals with or without T2DM. For these reasons, we feel that the retrospective design itself did not introduce considerable information bias into the associations under investigation.

\section{Confounding}

Bias due to confounding occurs when an association between a determinant and an outcome is mixed or blurred by a third variable, that is usually associated with the determinant, and causally related to the outcome, but not in the causal pathway between determinant and outcome. In all studies in this thesis, we have adjusted for several potential confounding variables relevant for the associations observed, as both the individuals of the CODAM study and the patients with cirrhosis were well characterized with regard to the most important aetiological risk factors at baseline, based on prior knowledge. However, as always in observational research, we cannot exclude the possibility of residual confounding. Unmeasured confounding factors / processes may still be responsible for (part of) the associations observed in this thesis, leading to an overestimation of effect sizes reported here.

An important issue in whether to adjust or not for a potential confounding covariate is whether the variable is believed to be part of the causal pathway between the 
determinant and outcome under study. If the variable is believed to be an intermediate in the pathway between determinant and outcome based on prior knowledge, it can be considered a mediator, and adjustment for this mediator is not desirable when studying the association between determinant and outcome. However, it can be interesting to adjust for the mediator in the face of mediation analyses (Chapters 2.5, 3.2, 4.2, 4.3, 4.5). If the covariate is believed to be a factor associated with the determinant, but not in the causal pathway, it is a confounding variable, and adjustment for this variable is necessary to reduce bias by confounding. The third possibility, which is often the case in this thesis, is that the covariate is a proximal factor that is causally related to both determinant and outcome under study (e.g. waist circumference). Both from the point of confounding and from the view of mediation analyses, adjustment for such a proximal factor is warranted to estimate the independent association between determinant and outcome (16-19). However, as we have shown in Chapter 2.5, adjustment for proximal causal factors that are measured with more precision (i.e. less random error) than the determinant under study may attenuate or even obscure the true association of the determinant with the outcome.

In summary, adjustment for a mediator or proximal causal factor with less measurement error than the determinant may lead to an underestimation of effect sizes, but failure to adjust for a confounder may lead to an overestimation of effect sizes. Mathematically, adjustment for a mediator, confounder, or proximal causal factor is identical, so that the distinction cannot be made based on the data. Therefore, investigators should discuss the interpretation of adjustments carefully from different points of view and based on prior knowledge, as we have done in studies in the present thesis (e.g. in Chapter 4.3 and 4.4).

\section{Causality in aetiological epidemiology}

The studies in this thesis were conducted to explore novel, aetiological mechanisms that may lead to insulin resistance and T2DM in humans. All studies were performed on observational data. From a causal point of view, observational data yield "nothing more than statistically significant associations between two variables". The nature of these data does not allow to draw definite conclusions on causality, nor does it rule out reverse causality. Therefore, any inferences on cause and effect drawn from these studies should be made with caution. However, proof of causality was not the purpose of this thesis. Most of the studies in this thesis were first-ever studies in humans, and were undertaken to investigate whether the hypotheses derived from extensive animal or in vitro data could be confirmed in humans in vivo (Chapter 3 and 4). The proposed directions of associations under investigation were therefore always corroborated by (stronger) causal evidence from knockout animals, or in vitro experiments, as discussed in these chapters. 


\section{Clinical implications and future perspectives}

\section{Iron metabolism}

In Chapter 3, we have shown that several markers of iron metabolism are associated with insulin resistance in several tissues, as well as with glucose tolerance. These findings suggest that body iron stores and/or iron-metabolism-related factors may contribute to the induction of insulin resistance early in the pathogenesis of T2DM, compatible with several animal and in vitro data on the effects of iron on glucose and lipid metabolism (20-25). Our studies are among the first that show that iron metabolism may also be related to adipocyte metabolism $(22,24)$, and altogether, these studies suggest that iron metabolism, and its effects on adipose tissue, the liver, and muscle, constitutes a novel and interesting subject for future research, both in vitro and in vivo.

Body iron stores can easily be influenced by low-cost interventions as phlebotomies or dietary interventions, and therefore represent interesting therapeutic options in the treatment, or perhaps prevention of T2DM. It has been shown long ago that iron depletion by phlebotomy improves diabetic metabolic control in $35-45 \%$ of patients, in the treatment of hereditary hemochromatosis (26). A small randomized controlled trial in individuals with T2DM and increased ferritin levels $(>200 \mathrm{ng} / \mathrm{mL})$, but no evidence of hereditary hemochromatosis, showed that bloodletting of $1500 \mathrm{~mL}$ led to improved insulin sensitivity and a decrease in glycated haemoglobin $\left(\mathrm{HbA}_{1 \mathrm{c}}\right)$ of 0.6 (27). Other, non-randomized, studies in patients with NAFLD or impaired glucose tolerance (and high-to-normal ferritin) have also shown that normalisation of serum ferritin by phlebotomy led to improved HOMA-IR and glucose tolerance $(24,25,28,29)$. In addition to data on phlebotomies, a small case series showed that administration of the iron chelator deferoxamine led to similar improvements in metabolic control (i.e. decrease in $\mathrm{HbA}_{1 \mathrm{c}}$ of $0.5-0.6$ ) in patients with T2DM and hyperferritinemia, but not in those with normal ferritin levels (30). Whether iron depletion also affects adipocyte insulin resistance, remains to be investigated, but animal studies have shown decreased triglyceride and NEFA levels after dietary iron restriction (23), and phlebotomies in human individuals with high-normal ferritin have recently been shown to increase serum adiponectin (24).

About $7 \%$ of unselected patients with T2DM have elevated ferritin levels (31), and median serum ferritin in individuals with T2DM in our (selected) cohort was $216 \mathrm{ng} / \mathrm{mL}$ (IQR 127-340). As such, there is probably a large number of patients that might profit from iron-lowering interventions as an alternative or an addition to current antihyperglycaemic treatment. Additional randomized controlled studies are therefore necessary to evaluate the effect of iron depletion on outcomes such as glycaemic control, but also on hard outcomes such as complications of T2DM and the metabolic syndrome, including CVD and cirrhosis. 


\section{The complement system}

The complement system is a relatively novel player in the field of metabolic diseases such as T2DM and (N)AFLD. In Chapters 4.2 and 4.3, we have shown that (changes in) plasma C3 levels in humans may reflect (a progression of) metabolic dysregulation, including low-grade inflammation and insulin resistance, that eventually leads to abnormalities in glucose tolerance and frank T2DM. Plasma C3 levels may therefore serve as a prognostic marker to identify individuals with an increased risk of metabolic diseases, who might benefit from lifestyle interventions such as calorie restrictions, dietary pattern changes or exercise programs, or perhaps from medical treatment. Dietary interventions and/or the accompanying weight loss have been shown to lower plasma C3 levels $(32,33)$. Moreover, rosiglitazone treatment has been shown to partially reduce postprandial plasma C3 levels (34), but treatment with metformin had no effect (35). Finally, statin treatment has been shown to reduce fasting and postprandial C3 levels $(36,37)$. It is, however, currently unknown whether the observed decreases in C3 are caused by these interventions themselves, or rather reflect improvement of the underlying disorders in glucose and lipid metabolism and low-grade inflammation. It is currently also unresolved to which extent lowering plasma C3 levels will actually result in a decreased risk of metabolic diseases. Future (clinical) studies will have to investigate whether monitoring and using plasma C3 as a risk marker is clinically useful in the treatment or prevention of metabolic diseases. Further in vivo and in vitro studies are also needed to investigate the effects of cytokines, insulin, NEFAs and triglycerides on C3 transcription and production in hepatocytes and adipocytes, and to identify the source(s) that contribute(s) to changes in circulating C3 in individuals at risk for metabolic diseases.

Besides a potential risk marker, plasma C3, or downstream components of complement activation may also actively contribute to metabolic diseases such as T2DM, the metabolic syndrome, and fatty liver disease by inducing and/or perpetuating the low-grade inflammatory response that is believed to play a major role in these diseases (Chapter 1). We have shown in two pilot studies (Chapter 4.4 and 4.5 ) that complement activation may contribute to the spectrum of AFLD and NAFLD in humans. Moreover, animal studies in which the effects of C3a and C5a were blocked, have shown improvements in (systemic) inflammation, glucose and lipid metabolism, hepatic steatosis, and hepatocellular damage (38-41). The effects of complement antagonists / inhibitors on metabolism in humans are however still unknown, as these agents have only recently been developed and applied in autoimmune diseases with a much higher degree of inflammation than the low-grade inflammation in obesity and T2DM (42). In addition, these novel agents are (yet) too expensive to be used for large-scale prevention or chronic treatment. Therefore, first, it remains to be elucidated in future studies whether complement activation products can causally contribute to the development of insulin resistance and fatty liver disease, before experimental trials with these agents are undertaken to influence the development of metabolic diseases. 


\section{Liver cirrhosis}

Contrary to the results of basic aetiological research in the previous chapters, the findings from Chapter 5 are more easily translated to clinical practice. We observed that about $40 \%$ of patients with liver cirrhosis also have T2DM, and that this high prevalence could not be explained by traditional risk factors for T2DM in these patients (Chapter 5.2). These findings suggest that liver cirrhosis, or perhaps chronic liver diseases in general, constitutes an important independent risk factor for the development of T2DM. On the other hand, our results are also compatible with the hypothesis that T2DM in the presence of chronic liver diseases may accelerate the progression of fibrosis to cirrhosis.

The clinical significance of this high prevalence of T2DM in patients with cirrhosis is yet unclear, as our data add up to a number of prospective and retrospective with conflicting results on mortality (Chapter 5.3) (43-47). However, our study consisted of 226 patients with a median follow-up of 6.2 years and no less than 118 deaths, where previous studies were generally small $(n=<100)(45-47)$, had a low number of events $(n=<20)(44-46)$, and/or included patients with longstanding cirrhosis at baseline $(43,44)$. The fully adjusted hazard ratio of $0.96(95 \% \mathrm{Cl} 0.62-1.49)$ for T2DM observed in our retrospective study suggests no large difference in survival between patients with and without T2DM, but does not exclude a small prognostic effect of T2DM on mortality, such as observed by Bianchi in the largest retrospective cohort study published thus far (143 events, HR 1.18; 95\%Cl 1.03-1.37) (43).

We did show that T2DM is an independent determinant of incident SBP in patients with cirrhosis, in agreement with higher rates of respiratory or urinary tract infections reported in patients with cirrhosis and T2DM $(48,49)$. When confirmed by other studies, this finding may have two clinical implications. First, T2DM in patients with cirrhosis may serve as a marker to identify individuals at increased risk for bacterial infections, who should be monitored very closely, and who might benefit from antibiotic prophylaxis. Currently, such prophylaxis is only offered to patients with gastro-intestinal bleeding or ascites and advanced cirrhosis (50). Second, the presence of T2DM in patients with cirrhosis may actually be a (modifiable) risk factor that further increases the susceptibility for (bacterial) infections, leading to more frequent hospitalizations and (antibiotic) treatments, with considerable health care costs. As such, antihyperglycaemic treatment may theoretically reduce the incidence of infections. At this time there are no randomized controlled trials on treatment of T2DM in patients with cirrhosis, but a few small observational studies suggest that good glycaemic control or treatment with metformin might be associated with decreased mortality $(51,52)$. In order to establish T2DM as a definitive prognostic marker or factor, either larger, prospective cohort studies with sufficient events and long-term follow-up or randomized controlled trials on antihyperglycaemic treatment should be performed in the future. Such studies with structural follow-up could also provide insight in whether T2DM is associated with (hospitalization for) a variety of bacterial infections, 
including SBP, and with progression of Child-Pugh and MELD scores. If there is indeed a clinically relevant effect of T2DM on morbidity and mortality of patients with cirrhosis, this population should be screened for T2DM, given its high prevalence, and T2DM should be treated aggressively. However, medical treatment of T2DM in patients with cirrhosis is not without risks (Chapter 5.1), and the current evidence to test and treat for T2DM in patients with cirrhosis is not yet conclusive. Therefore, the potential benefits and harms of treatment of T2DM in patients with cirrhosis should be carefully considered in each patient individually. 


\section{References}

1. Forouhi NG, Harding AH, Allison M, Sandhu MS, Welch A, Luben R, Bingham S, Khaw KT, Wareham NJ: Elevated serum ferritin levels predict new-onset type 2 diabetes: results from the EPIC-Norfolk prospective study. Diabetologia 2007;50:949-956

2. Duncan BB, Schmidt MI, Pankow JS, Bang H, Couper D, Ballantyne CM, Hoogeveen RC, Heiss G: Adiponectin and the development of type 2 diabetes: the atherosclerosis risk in communities study. Diabetes 2004;53:2473-2478

3. Nationaal Kompas Volksgezondheid [online] [article online], 2013. Accessed October 20132013

4. International Diabetes Federation. Idf diabetes atlas: the global burden, 5 th edition [online] [article online], 2011. Accessed September 2013

5. Grimes DA, Schulz KF: Bias and causal associations in observational research. Lancet 2002;359:248-252

6. Cole SR, Platt RW, Schisterman EF, Chu H, Westreich D, Richardson D, Poole C: Illustrating bias due to conditioning on a collider. Int J Epidemiol 2010;39:417-420

7. Greenland S: Randomization, statistics, and causal inference. Epidemiology 1990;1:421-429

8. Weuve J, Tchetgen Tchetgen EJ, Glymour MM, Beck TL, Aggarwal NT, Wilson RS, Evans DA, Mendes de Leon CF: Accounting for bias due to selective attrition: the example of smoking and cognitive decline. Epidemiology 2012;23:119-128

9. Baan CA, van Baal PH, Jacobs-van der Bruggen MA, Verkley H, Poos MJ, Hoogenveen RT, Schoemaker CG: [Diabetes mellitus in the Netherlands: estimate of the current disease burden and prognosis for 2025]. Ned Tijdschr Geneeskd 2009;153:1052-1058

10. Copeland KT, Checkoway H, McMichael AJ, Holbrook RH: Bias due to misclassification in the estimation of relative risk. Am J Epidemiol 1977;105:488-495

11. Jurek AM, Greenland S, Maldonado G, Church TR: Proper interpretation of non-differential misclassification effects: expectations vs observations. Int J Epidemiol 2005;34:680-687

12. Szczepaniak LS, Nurenberg P, Leonard D, Browning JD, Reingold JS, Grundy S, Hobbs HH, Dobbins RL: Magnetic resonance spectroscopy to measure hepatic triglyceride content: prevalence of hepatic steatosis in the general population. Am J Physiol Endocrinol Metab 2005;288:E462-468

13. Bonora E, Targher G, Alberiche M, Bonadonna RC, Saggiani F, Zenere MB, Monauni T, Muggeo M: Homeostasis model assessment closely mirrors the glucose clamp technique in the assessment of insulin sensitivity: studies in subjects with various degrees of glucose tolerance and insulin sensitivity. Diabetes Care 2000;23:57-63

14. Bohte AE, van Werven JR, Bipat S, Stoker J: The diagnostic accuracy of US, CT, MRI and 1H-MRS for the evaluation of hepatic steatosis compared with liver biopsy: a meta-analysis. Eur Radiol 2011;21:87-97

15. Bravo AA, Sheth SG, Chopra S: Liver biopsy. N Engl J Med 2001;344:495-500

16. Robins JM: Data, design, and background knowledge in etiologic inference. Epidemiology 2001;12:313320

17. MacKinnon DP, Krull JL, Lockwood CM: Equivalence of the mediation, confounding and suppression effect. Prev Sci 2000;1:173-181

18. Mackinnon DP, Fairchild AJ: Current Directions in Mediation Analysis. Curr Dir Psychol Sci 2009;18:16

19. Shrout PE, Bolger N: Mediation in experimental and nonexperimental studies: new procedures and recommendations. Psychol Methods 2002;7:422-445

20. Green A, Basile R, Rumberger JM: Transferrin and iron induce insulin resistance of glucose transport in adipocytes. Metabolism 2006;55:1042-1045

21. Rumberger JM, Peters T, Jr., Burrington C, Green A: Transferrin and iron contribute to the lipolytic effect of serum in isolated adipocytes. Diabetes 2004;53:2535-2541

22. Dongiovanni P, Ruscica M, Rametta R, Recalcati S, Steffani L, Gatti S, Girelli D, Cairo G, Magni P, Fargion $\mathrm{S}$, Valenti L: Dietary iron overload induces visceral adipose tissue insulin resistance. Am J Pathol 2013; 182:2254-2263

23. Cooksey RC, Jones D, Gabrielsen S, Huang J, Simcox JA, Luo B, Soesanto Y, Rienhoff H, Abel ED, McClain DA: Dietary iron restriction or iron chelation protects from diabetes and loss of beta-cell function in the obese (ob/ob lep-/-) mouse. Am J Physiol Endocrinol Metab 2010;298:E1236-1243 
24. Gabrielsen JS, Gao Y, Simcox JA, Huang J, Thorup D, Jones D, Cooksey RC, Gabrielsen D, Adams TD, Hunt SC, Hopkins PN, Cefalu WT, McClain DA: Adipocyte iron regulates adiponectin and insulin sensitivity. J Clin Invest 2012;122:3529-3540

25. Fargion S, Dongiovanni P, Guzzo A, Colombo S, Valenti L, Fracanzani AL: Iron and insulin resistance. Aliment Pharmacol Ther 2005;22 Suppl 2:61-63

26. Davis WD, Jr., Arrowsmith WR: The treatment of hemochromatosis by massive venesection. Ann Intern Med 1953;39:723-734

27. Fernandez-Real JM, Penarroja G, Castro A, Garcia-Bragado F, Hernandez-Aguado I, Ricart W: Blood letting in high-ferritin type 2 diabetes: effects on insulin sensitivity and beta-cell function. Diabetes 2002;51:1000-1004

28. Valenti L, Fracanzani AL, Dongiovanni P, Bugianesi E, Marchesini G, Manzini P, Vanni E, Fargion S: Iron depletion by phlebotomy improves insulin resistance in patients with nonalcoholic fatty liver disease and hyperferritinemia: evidence from a case-control study. Am J Gastroenterol 2007;102:1251-1258

29. Valenti L, Moscatiello S, Vanni E, Fracanzani AL, Bugianesi E, Fargion S, Marchesini G: Venesection for non-alcoholic fatty liver disease unresponsive to lifestyle counselling--a propensity score-adjusted observational study. QJM 2011;104:141-149

30. Cutler P: Deferoxamine therapy in high-ferritin diabetes. Diabetes 1989;38:1207-1210

31. O'Brien T, Barrett B, Murray DM, Dinneen S, O'Sullivan DJ: Usefulness of biochemical screening of diabetic patients for hemochromatosis. Diabetes Care 1990;13:532-534

32. Pomeroy C, Mitchell J, Eckert E, Raymond N, Crosby R, Dalmasso AP: Effect of body weight and caloric restriction on serum complement proteins, including Factor D/adipsin: studies in anorexia nervosa and obesity. Clin Exp Immunol 1997;108:507-515

33. Hernandez-Mijares A, Banuls C, Bellod L, Jover A, Sola E, Morillas C, Victor VM, Rocha M: Effect of weight loss on C3 and C4 components of complement in obese patients. Eur J Clin Invest 2012;42: 503-509

34. Tahiri Y, Karpe F, Tan GD, Cianflone K: Rosiglitazone decreases postprandial production of acylation stimulating protein in type 2 diabetics. Nutrition \& metabolism 2007;4:11

35. Carter AM, Bennett CE, Bostock JA, Grant PJ: Metformin reduces C-reactive protein but not complement factor C3 in overweight patients with Type 2 diabetes mellitus. Diabet Med 2005;22:12821284

36. Verseyden $\mathrm{C}$, Meijssen S, van Dijk H, Jansen H, Castro Cabezas M: Effects of atorvastatin on fasting and postprandial complement component 3 response in familial combined hyperlipidemia. J Lipid Res 2003;44:2100-2108

37. Sampietro T, Bigazzi F, Rossi G, Dal Pino B, Puntoni MR, Sbrana F, Chella E, Bionda A: Upregulation of the immune system in primary hypercholesterolaemia: effect of atorvastatin therapy. J Int Med 2005; 257:523-530

38. Mamane Y, Chung Chan C, Lavallee G, Morin N, Xu L, Huang J, Gordon R, Thomas W, Lamb J, Schadt EE, Kennedy BP, Mancini JA: The C3a anaphylatoxin receptor is a key mediator of insulin resistance and functions by modulating adipose tissue macrophage infiltration and activation. Diabetes 2009;58:20062017

39. Phieler J, Chung KJ, Chatzigeorgiou A, Klotzsche-von Ameln A, Garcia-Martin R, Sprott D, Moisidou M, Tzanavari T, Ludwig B, Baraban E, Ehrhart-Bornstein M, Bornstein SR, Mziaut H, Solimena M, Karalis KP, Economopoulou M, Lambris JD, Chavakis T: The Complement Anaphylatoxin C5a Receptor Contributes to Obese Adipose Tissue Inflammation and Insulin Resistance. J Immunol 2013; DOI 10.4049/jimmunol.1300038

40. Lim J, Iyer A, Suen JY, Seow V, Reid RC, Brown L, Fairlie DP: C5aR and C3aR antagonists each inhibit dietinduced obesity, metabolic dysfunction, and adipocyte and macrophage signaling. FASEB 2013;27: 822-831

41. Roychowdhury S, McMullen MR, Pritchard MT, Hise AG, van Rooijen N, Medof ME, Stavitsky AB, Nagy LE: An early complement-dependent and TLR-4-independent phase in the pathogenesis of ethanolinduced liver injury in mice. Hepatology 2009;49:1326-1334

42. Ricklin D, Lambris JD: Complement in immune and inflammatory disorders: therapeutic interventions. J Immunol 2013;190:3839-3847 
43. Bianchi G, Marchesini G, Zoli M, Bugianesi E, Fabbri A, Pisi E: Prognostic significance of diabetes in patients with cirrhosis. Hepatology 1994;20:119-125

44. Quintana JO, Garcia-Compean D, Gonzalez JA, Perez JZ, Gonzalez FJ, Espinosa LE, Hernandez PL, Cabello ER, Villarreal ER, Rendon RF, Garza HM: The impact of diabetes mellitus in mortality of patients with compensated liver cirrhosis-a prospective study. Ann Hepatol 2011;10:56-62

45. Hagel S, Bruns T, Herrmann A, Stallmach A, Schmidt C: Abnormal glucose tolerance: a predictor of 30day mortality in patients with decompensated liver cirrhosis. Z Gastroenterol 2011;49:331-334

46. Holstein A, Hinze S, Thiessen E, Plaschke A, Egberts EH: Clinical implications of hepatogenous diabetes in liver cirrhosis. J Gastroenterol Hepatol 2002;17:677-681

47. Moreau R, Delegue P, Pessione F, Hillaire S, Durand F, Lebrec D, Valla DC: Clinical characteristics and outcome of patients with cirrhosis and refractory ascites. Liver Int 2004;24:457-464

48. Diaz J, Monge E, Roman R, Ulloa V: Diabetes as a risk factor for infections in cirrhosis. Am J Gastroenterol 2008;103:248

49. Amato A, Precone DF, Carannante N, Brancaccio G, Stornaiuolo G, Galante D, Gaeta GB: [Prevalence and risk factors for bacteriuria in patients with cirrhosis]. Infez Med 2005;13:103-108

50. Fernandez J, Gustot T: Management of bacterial infections in cirrhosis. J Hepatol 2012;56 Suppl 1:S1-12

51. Nkontchou G, Cosson E, Aout M, Mahmoudi A, Bourcier V, Charif I, Ganne-Carrie N, Grando-Lemaire V, Vicaut $\mathrm{E}$, Trinchet JC, Beaugrand $\mathrm{M}$ : Impact of metformin on the prognosis of cirrhosis induced by viral hepatitis C in diabetic patients. J Clin Endocrinol Metab 2011;96:2601-2608

52. Kwon SY, Kim SS, Kwon OS, Kwon KA, Chung MG, Park DK, Kim YS, Koo YS, Kim YK, Choi DJ, Kim JH: Prognostic significance of glycaemic control in patients with HBV and HCV-related cirrhosis and diabetes mellitus. Diabet Med 2005;22:1530-1535 
Samenvatting voor niet ingewijden 


\section{Samenvatting voor niet ingewijden}

Diabetes mellitus, oftewel suikerziekte, is een van de meest voorkomende chronische ziekten. Wereldwijd hebben 346 miljoen mensen diabetes, en in Nederland zullen er in 2025 ongeveer 1,3 miljoen mensen diabetes hebben. Patiënten met diabetes hoeven niet per se klachten van een hoge suikerconcentratie te hebben, maar de ziekte leidt wel tot diverse complicaties, zoals blindheid, nierfalen, zenuwschade, en hart- en vaatziekten zoals hart- en herseninfarct, alsmede tot sterfte hieraan. Er wordt dan ook geschat dat in 2011 ongeveer 4,6 miljoen mensen wereldwijd zijn overleden aan de gevolgen van diabetes mellitus.

De meeste mensen (90-95\%) met suikerziekte hebben diabetes mellitus type 2 (DM2), waarbij verschillende weefsels (spier, vet, lever) verminderd gevoelig zijn voor de effecten van insuline (zogenaamde insulineresistentie). Op een gegeven moment kan het lichaam niet meer voldoende extra insuline produceren om deze insulineresistentie te boven te komen, en hierdoor wordt de suikerconcentratie in het bloed te hoog. Men spreekt van gestoorde glucosetolerantie wanneer de suikerconcentratie licht verhoogd is, en van DM2 wanneer de suikerconcentratie sterk verhoogd is. Insulineresistentie kan zich naast afwijkingen in de suikerbalans ook kenmerken door diverse andere afwijkingen in de stofwisseling, zoals te laag 'goed' cholesterol en te hoge vetten in het bloed, hoge bloeddruk, en vervetting van de lever, hetgeen non-alcoholische leververvetting genoemd wordt.

Hoe deze insulineresistentie precies veroorzaakt wordt, is nog niet helemaal opgehelderd, maar insulineresistentie treedt het vaakst op bij mensen met overgewicht of obesitas (zwaarlijvigheid), in het bijzonder wanneer het teveel aan vetweefsel zich rondom en in de buik bevindt. Ook is de laatste jaren steeds duidelijker geworden dat mensen met te veel vet in de buik een soort ontsteking in vet en lever doormaken, waarbij ook in het bloed deze ontstekingsstofjes licht verhoogd zijn. Dit wordt ook wel laaggradige ontsteking genoemd, om het te onderscheiden van de sterke ontstekingsreactie die men bijvoorbeeld ziet tijdens een infectie. Deze ontstekingsstofjes, alsmede verhoogde vetzuren in het bloed, worden verondersteld ten grondslag te liggen aan de insulineresistentie in DM2. Het is echter nog onduidelijk welke processen deze laaggradige ontsteking veroorzaken. Onderzoek naar de achterliggende mechanismen is daarom van belang, en kan mogelijk bijdragen aan nieuwe behandelingen of behandelstrategieën voor patiënten met $\mathrm{DM} 2$, dan wel preventieve maatregelen voor mensen met een sterk verhoogd risico om DM2 te ontwikkelen.

Het doel van dit proefschrift was dan ook om drie nieuwe mechanismen te bestuderen, die betrokken zouden kunnen zijn bij het ontstaan van insulineresistentie en DM2. Deze drie mechanismen zijn: ijzermetabolisme, het complement systeem en levercirrose. In Hoofdstuk 1 wordt de huidige kennis over het ontstaan van insulineresistentie en DM2 kort samengevat en de probleemstellingen geïntroduceerd. 
De probleemstellingen worden in dit proefschrift onderzocht met data van twee grote studies: de Cohort on Diabetes and Atherosclerosis studie (CODAM studie), en een cohort van patiënten met levercirrose uit Eindhoven. In Hoofdstuk $\mathbf{2}$ wordt in enkele deelhoofdstukken een gedetailleerde toelichting gegeven over de twee studiepopulaties die onderzocht zijn, de manier waarop wij insulineresistentie, ontsteking en levervet hebben gemeten, en de manier waarop de beschreven resultaten geïnterpreteerd kunnen worden.

In Hoofdstuk 3 hebben we de rol van ijzer, en van stofjes die betrokken zijn bij het metabolisme van ijzer, onderzocht in relatie tot insulineresistentie en glucosetolerantie. IJzer wordt in het lichaam opgeslagen in de vorm van ferritine, en gebonden aan transferrine in het bloed vervoerd. Verder komt ijzer in kleine hoeveelheden los in het bloed voor (niet-transferrine gebonden ijzer). Eerdere studies naar de betrokkenheid van ijzer bij DM2 hebben aangetoond dat hogere concentraties ferritine gerelateerd zijn aan een hogere mate van insulineresistentie (in het algemeen, dan wel in spierweefsel), en aan een grotere kans op het krijgen van DM2 (inleidend Hoofdstuk 3.1).

In Hoofdstuk 3.2 hebben wij het verband onderzocht tussen aan de ene kant de concentraties van alle stoffen die betrokken zijn bij het metabolisme van ijzer, en aan de andere kant de (geschatte) mate van insulineresistentie in het vetweefsel. We vonden dat hogere waarden voor alle vier de factoren (ferritine, transferrine, totaal ijzer, en niet-transferrine gevonden ijzer) gerelateerd waren aan een hogere mate van insulineresistentie in vetweefsel, en dat hogere concentraties ferritine en transferrine gerelateerd waren aan lagere concentraties adiponectine, een stofje geproduceerd door vetweefsel dat lijkt te beschermen tegen insulineresistentie en DM2.

In Hoofdstuk 3.3 hebben we de data van de vorige studie uit Hoofdstuk $\mathbf{3 . 2}$ uitgebreid met follow-updata van de proefpersonen die 7 jaar later wederom werden gemeten, alsmede met nieuwe metingen van insuline in het bloed. Met deze nieuwe data laten we zien dat de concentraties ferritine en transferrine op het begin van de studie gerelateerd zijn aan hogere mate van insulineresistentie gedurende de studieperiode van 7 jaar. Bovendien vinden we deze relaties met zowel insulineresistentie in het hele lichaam (met name spierweefsel), met insulineresistentie in vetweefsel, als ook met een nieuwe maat voor insulineresistentie in de lever. Ten slotte tonen we aan dat hogere concentraties ferritine en transferrine samenhangen met een afwijkende glucosetolerantie gedurende 7 jaar follow-up. Samenvattend suggereren de resultaten van deze twee studies dat de hoeveelheid ijzer in het lichaam, dan wel factoren als ferritine en transferrine, betrokken kunnen zijn bij het ontstaan van insulineresistentie in meerdere weefsels, zoals spier, vet en lever, hetgeen uiteindelijk leidt tot gestoorde glucosetolerantie en DM2. 
In Hoofdstuk 4 hebben we het complement systeem bekeken in relatie tot obesitas, insulineresistentie, laaggradige ontsteking, DM2 en leververvetting in vier studies binnen de CODAM studie. Het complement systeem is een groep eiwitten in het bloed (genaamd C1 tot en met C9) dat deel uitmaakt van het afweersysteem, en betrokken is bij ontstekingsreacties. Als dit systeem geactiveerd raakt, vindt een opeenvolging van activerende reacties plaats, die in eerste instantie leiden tot het splitsen van de factor C3 in C3b en het kleine C3a. Dit C3a werkt als een signaalmolecuul en trekt witte bloedcellen naar de plek van de ontsteking toe, activeert ze, en verhoogt de productie van allerlei ontstekingsstofjes. C3b activeert de volgende factoren van het complementsysteem, waarop een geheel van eiwitten gevormd wordt, genaamd C5b-C9, oftewel het terminale complementcomplex. Dit complex veroorzaakt in kleine hoeveelheden ook een toename van ontsteking, en kan in grote hoeveelheden zelfs direct cellen of bacteriën aanvallen. De resultaten van enkele studies naar het complement systeem en insulineresistentie dan wel leververvetting worden besproken in het inleidende Hoofdstuk 4.1, maar studies bij mensen zijn nog maar weinig voorhanden.

We weten dat dikkere mensen hogere concentraties C3 in het bloed hebben, maar de oorzaak hiervoor is niet helemaal duidelijk. In Hoofdstuk 4.2 onderzochten we daarom met geavanceerde statistische analyses welke factoren hierin een rol konden spelen. We vonden dat vet-gerelateerde laaggradige ontsteking het verband tussen obesitas en hogere C3-waarden voor 20-25\% kan verklaren, en dat vet-gerelateerde insulineresistentie nog eens 40-55\% kon verklaren. In Hoofdstuk $\mathbf{4 . 3}$ hebben we vervolgens gefocust op het verband tussen $\mathrm{C} 3$ en insulineresistentie. In deze studie vonden we dat een toename in C3-concentraties in het bloed gepaard ging met een toename in de mate van insulineresistentie in zowel spier, lever, als vet gedurende de 7 jaren follow-up. Bovendien hadden personen met hogere C3-waarden op het begin van de studie een grotere kans om DM2 te ontwikkelen tijdens de daaropvolgende 7 jaren dan personen met lagere C3-waarden. De resultaten van onze twee studies suggereren dat hoge C3-waarden in het bloed een weerspiegeling zijn van diverse processen, waaronder insulineresistentie en ontsteking, die uiteindelijk leiden tot glucoseintolerantie en DM2. Of C3 ook daadwerkelijk de ontsteking en insulineresistentie veroorzaakt, dient nog nader onderzocht te worden.

In Hoofdstuk 4.4 en $\mathbf{4 . 5}$ onderzochten we de rol van activatie van het complement systeem in relatie tot leververvetting en leverschade (dit laatste wordt gereflecteerd door hogere concentraties leverenzymen in het bloed). In de eerste studie (Hoofdstuk 4.4) vonden we dat hogere C3a-waarden in het bloed gerelateerd waren aan hogere waarden levervet en hogere leverenzymen, en dat dit verband met name aanwezig was in personen die veel alcohol dronken (>2 glazen/dag voor vrouwen, $>3$ glazen/dag voor mannen). Verder zagen we dat deze verbanden voor $30 \%$ verklaard konden worden door het ontstekingsstofje tumornecrosefactor-alfa (TNF- $\alpha$ ). In de tweede studie (Hoofdstuk 4.5 ) bleek dat ook de concentraties C5b-C9, oftewel het 
terminale complementcomplex, in het bloed gerelateerd waren aan hogere mate van leververvetting en hogere leverenzymen, en ditmaal was de relatie minder afhankelijk van het alcoholgebruik. Samenvattend laten deze twee studies voor het eerst zien dat complementactivatie, met ontstaan van $\mathrm{C3a}$ en $\mathrm{C} 5 \mathrm{~b}-\mathrm{C} 9$, mogelijk kan bijdragen aan leververvetting en levercelschade bij mensen.

Hoofdstuk 5 bevat twee klinische studies naar de relatie tussen DM2 en levercirrose. Levercirrose is verlittekening van de lever ten gevolge van langdurige leverschade, bijvoorbeeld door overmatig alcoholgebruik, virale hepatitis B of C infecties, of non-alcoholische leververvetting bij zwaarlijvige (obese) personen. Bij toenemende verlittekening van de lever ontstaat leverfalen, met complicaties als bloedingen (in het maag-darmkanaal maar ook elders), infecties, coma, nierfalen en leverkanker, waardoor deze patiënten een zeer slechte prognose hebben. De lever is een essentieel orgaan in de huishouding van suiker, en patiënten met cirrose hebben vaker DM2 (inleidend Hoofdstuk 5.1). Of dit komt door de leverziekte zelf, of door het vaak voorkomen van obesitas is nog niet geheel duidelijk.

In Hoofdstuk 5.2 vergeleken wij daarom de mate waarin DM2 voorkwam bij patiënten met cirrose met een controlegroep van patiënten met maagklachten zonder lichamelijk aantoonbare oorzaak. Van de patiënten met cirrose had 37\% DM2, in tegenstelling tot $7 \%$ van de gezonde controlegroep. Dit verband werd niet verklaard door leeftijd, obesitas of familiaire belasting voor DM2.

Een interessante vraag is of DM2 bij deze patiënten ook tot complicaties en een hogere sterfte leidt, zoals eerder beschreven bij patiënten met DM2 zonder cirrose door hart- en vaatziekten. In Hoofdstuk $\mathbf{5 . 3}$ onderzochten wij daarom wat het effect van DM2 was op de overleving van patiënten met cirrose. In deze studie was de aanwezigheid van DM2 niet gerelateerd aan een slechtere prognose, hoewel een klein effect niet geheel uitgesloten is. Wel vonden we dat patiënten met DM2 een hogere kans hadden op het ontwikkelen van een spontane bacteriële peritonitis, een zeer gevaarlijke en potentieel dodelijke infectie van het buikvlies waarvoor behandeling met antibiotica in het ziekenhuis nodig is. Onze resultaten suggereren dat DM2 veel voorkomt bij patiënten met cirrose, waarschijnlijk leidt tot meer bacteriële infecties, en mogelijk leidt tot een hogere sterfte, hoewel dat laatste niet in onze studie bewezen kon worden. In elk geval hebben deze patiënten, door de toch al slechte prognose van de leverziekte, geen verhoogde kans op sterfte door de reguliere hart- en vaatziekten veroorzaakt door DM2.

Tot slot worden de belangrijkste bevindingen van dit proefschrift in Hoofdstuk 6 samengevat en bediscussieerd. Hierbij worden nog diverse kanttekeningen geplaatst met betrekking tot de studiepopulaties en de gebruikte methoden, en hoe de resultaten hierdoor beïnvloed zouden kunnen zijn. In het kort ondersteunen de studies in dit proefschrift de hypothesen dat ijzermetabolisme, het complementsysteem dan 
wel complementactivatie, en levercirrose alle drie factoren zijn die kunnen bijdragen aan het ontstaan van insulineresistentie en uiteindelijk DM2. De toekomst zal moeten uitwijzen of wij deze processen kunnen beïnvloeden, maar aleer het zover is, zullen toekomstige studies de bijdrage van deze nieuwe mechanismen verder moeten ophelderen. 
Dankwoord 


\section{Dankwoord}

Eindelijk is het dan zover, en mag ik de laatste hand leggen aan dit proefschrift. Net als bij de laatste artikelen en het samenstellen van het boekje heb ik een rustige nachtdienst uitgekozen om deze bladzijden te gaan typen. Klaarwakker en met een bekertje dampende koffie op het bureau probeer ik om vier uur's nachts na te gaan wie ik allemaal wil bedanken voor de afgelopen jaren.

Allereerst wil ik de leden van mij promotieteam bedanken.

Prof. dr. C.D.A. Stehouwer, beste Coen, ik wil je allereerst bedanken voor de mogelijkheid die je mij hebt gegeven om te promoveren in een samenwerkingsverband tussen Eindhoven en Maastricht. Daarbij heb ik genoten van het vertrouwen en de zelfstandigheid waarmee ik heb mogen werken, en de vrijheid om dit meer metabole onderwerp uit te werken tussen al het vasculaire onderzoek dat onder jouw hoede plaatsvindt. Ondanks de afstand heb ik ontzettend veel van je geleerd, zowel inhoudelijk met betrekking tot het schrijven van een logisch verhaal, maar zeker ook in de manier waarop ik moet omgaan met editors en reviewers van tijdschriften. Ik weet dat bij dat laatste nog steeds ruimte voor verbetering is, aangezien ik de commentaren van reviewers meer emotioneel dan rationeel benader. Ookhetaanelkaarschrijvenvan deNederlandsetaalzalikwaarschijnlijknooitonderdekniekrijgen. Coen, ik kan niet in woorden uitdrukken hoe prettig het is om onmiddellijk maar zeer secuur beantwoordde emails, zelfs op de zondagmiddag, van je te ontvangen, waarmee knopen doorgehakt worden en je als promovendus vooruit kan.

Dr. M.M.J. van Greevenbroek, beste Marleen, zonder jou waren de meeste projecten in dit proefschrift nooit van de grond gekomen. Hoewel je opgezadeld werd met een promovendus op afstand in plaats van een kamer verder, heb ik ontzettend genoten van de tijd en moeite die je hebt gestoken in mijn promotietraject. Ik denk met plezier terug aan de avonden in mijn eerste jaar onderzoek, waarbij we hypothesen en ideeën uitwisselden, tot we erachter kwamen dat de zon allang onder was gegaan en we nog allebei terug naar huis moesten. Je was (voor mij) de eerste die de afkorting SBP bij patiënten met cirrose koppelde aan "systolic blood pressure". Door de jaren heen heb je altijd een grote rol gehad in het secuur doornemen van alle manuscripten, weer nieuwe analyses verzinnen, herformuleren van lastige zinnen, tot het opsturen naar de tijdschriften. Onder jouw begeleiding heb ik helemaal mezelf kunnen zijn, en heb ik met jou over alles kunnen praten, zelfs over de verscheidenheid aan Duitse feestmuziek op Mallorca.

Dr. B. Bravenboer, beste Bert. Ik wil je bedanken voor de manier waarop je bij mij het doen van onderzoek gestimuleerd hebt, al tijdens mijn WESP stage. Ik ben vereerd dat je mij een geschikte kandidaat vond om Hanneke op te volgen als $2^{\mathrm{e}}$ AGIKO in het 
Catharina Ziekenhuis. Onze samenwerking heeft daarbij ook nog twee mooie klinische case-reports opgeleverd. Daarnaast heb je mij weten te boeien voor het mooie vak van de interne geneeskunde, en heb ik ook als clinicus veel van je mogen leren. Ik betreur de manier waarop je in ons ziekenhuis afscheid hebt moeten nemen, maar ben blij dat ik je nog steeds als copromotor aan mijn zijde heb.

De leden van de beoordelingscommissie, prof. dr. T. Unger, prof. dr. P.C. Dagnelie, dr. G. Koek, prof. dr. P. Schrauwen, en prof. dr. C.J. Tack wil ik graag bedanken voor de tijd en moeite die jullie in het grondig doorlezen van mijn proefschrift hebben gestoken. Dan zijn er nog een aantal personen betrokken bij dit proefschrift die ik ook wil bedanken.

Prof. dr. C.G. Schalkwijk, beste Casper. Wij hebben niet veel fysiek contact gehad, maar toch heb je alle manuscripten grondig doorgelezen en van commentaar voorzien. Ik wil je bedanken voor de gastvrijheid die ik, als buitenstaander, heb genoten tijdens de congressen, labuitjes, personeelsdagen, en kerstdiners bij jou thuis in Banholt (met natuurlijk ook dank aan Hermine).

Dr. I. Ferreira, beste Isabel, ik wil je bedanken voor de tip-top databases die ik van je heb mogen ontvangen, de statistische commentaren, met name over mediatie analyses, en de interessante methodologische discussies van de afgelopen jaren, waar ik veel van geleerd heb.

De co-auteurs van de CODAM artikelen dr. C.J. van der Kallen, prof. dr. E.J. Feskens, dr. E.H. Jansen, jullie wil ik bedanken voor het grondig lezen van alle artikelen uit de CODAM studie, alsmede het geven van commentaren en aanvullingen hierop.

Prof. dr. J.W.R. Twisk, beste Jos, ik wil je bedanken voor de cursussen regressie analyse die ik in het kader van de Masteropleiding Epidemiologie bij jou gevolgd heb. De manier waarop jij iets extreems complex weet uit te leggen op kinderniveau, en daarbij steeds drie stappen voor te denken op je publiek, en discussie en interactie te gebruiken in je onderwijsvormen, is didactisch gezien een genot om bij te wonen. Ik was dan ook zeer vereerd om jou als stagebegeleider te hebben, en heb ongeloofelijk veel geleerd van je manier van denken.

Prof. dr. H.P. Sauerwein, beste Hans, jij bent voornamelijk in het begin betrokken geweest als inhoudsdeskundige toen ik als WESP-student artikelen wilde schrijven over diabetes en cirrose. Het verschil in kennis en ervaring tussen ons moet enorm geweest zijn, maar desalniettemin heb ik met jou zeer vruchtbaar van gedachten kunnen wisselen. Ook heb je me bij de eerste rebuttals meerdere malen goed geholpen en 
begeleid, waarmee de basis werd gelegd voor het kritisch doen en schrijven van wetenschappelijk onderzoek. Dank hiervoor!

Dr. H.J.B.H. Beijers, beste Hanneke, ook jij hebt mij als WESP-student enorm geholpen met het schrijven van mijn eerste artikel, alvorens dit naar de professoren opgestuurd werd, alsmede met statistische wissewasjes als SPSS weer eens vast liep. Vol trots heb ik als paranimf jouw promotie meegemaakt, en nu mag ik er zelf ook aan geloven. Ik zou hier dan ook niet kunnen staan zonder jou als paranimf naast mij. Ik ga je missen in het Catharina, maar zal je zeker nog wel eens tegen het lijf lopen, om te horen wat voor top-endocrinoloog je geworden bent.

Geachte dr. J. Curvers, beste Joyce, ik wil je bedanken voor het enthousiasme waarmee je meegeschreven hebt aan een van mijn projecten. Ik weet dat als ik in ons ziekenhuis het lab nodig heb, ik via jou nooit met lege handen thuis kom.

Dan wil ik de gastro-enterologen van het Catharina Ziekenhuis bedanken die bij dit project betrokken zijn geweest, dr. E.J. Schoon, en dr. P. Friederich. Beste Erik, ik vond het prettig dat ik als student altijd bij je binnen kon lopen om ideeën te overleggen en met een stel mooie antwoorden en met een mooie portie zelfvertrouwen weer de deur uit te gaan. Beste Pieter, ik waardeer je enorm als co-auteur bij mijn $2^{\mathrm{e}}$ cirrose stuk, en ook als een bedreven clinicus, waarvan ik veel heb mogen leren tijdens mijn MDL-stage, en niet alleen op medisch inhoudelijk vlak.

Dr. W.G. Peters, beste Wim, tijdens mijn coschap interne heeft u mij eruit gevist en voorgesteld voor een combinatiestage in jaar 6 van mijn opleiding, waarna het balletje voor de AGIKO constructie nadien al snel ging rollen. Ook vond ik het een mooie ervaring om met $u$ onderzoek te doen naar near-peer teaching in de interne geneeskunde middels een kleine RCT, waarvan ik methodologisch enorm veel heb geleerd. Ook de komende jaren in de kliniek hoop ik nog veel van u te leren.

Beste Bas, wij hebben de afgelopen jaren dezelfde strijd gestreden en methodologisch lief en leed gedeeld, ook al zaten we niet in dezelfde onderzoekslijn. Desalniettemin, onze boekjes zijn er nu allebei, en ik verheug me op jouw promotie. Ik ben je enorm dankbaar voor alle discussies die wij in de gang, tijdens de lunch en op congres, met name de WEON, hebben gehouden, en voor hoofdstuk 2.5 dat daaruit voort is gekomen. Ik heb genoten van ons midweekje Schotland tijdens de ADDDG, en van de enorme hopen vlees die we daar samen weggezet hebben. Als AIOS zullen wij elkaar zeker nog terugzien, en wie weet ook als co-auteur... 
Beste Tiny, dank je voor al het werk dat je hebt verricht in de afronding van dit proefschrift! Zonder jou had het nog zeker een half jaar geduurd voordat alles op de juiste plaats stond. Ik bewonder de precisie waarmee je werkt, en het enthousiasme waarmee je het boekje in elkaar hebt gestoken.

Mijn collega's van de interne geneeskunde in het Catharina Ziekenhuis, internisten en (ex) arts-assistenten, jullie zijn te talrijk om op te noemen, en door de jaren heen ook zo van samenstelling veranderd dat ik er makkelijk een paar zou vergeten als ik ze allemaal moest opnoemen. Jullie hebben mij als destijds als niet klinisch werkzame collega in de groep opgenomen, en wel voor $100 \%$. Jullie zullen je wel eens afgevraagd hebben waar ik de hele dag mee bezig was op mijn computer op de poli, maar zie hier dan eindelijk het resultaat. Gelukkig werd dit soms wel eenzame wetenschappelijke werk afgewisseld door bezoekjes van assistenten uit de kliniek, om moeilijke patiënten te beschouwen, eens lekker te klagen, of te bedenken waar we vrijdag na de borrel konden gaan eten. Ook tijdens de opleiding voel ik me perfect op mijn plaats bij jullie. Tijdens de jaarlijkse skivakanties hebben jullie de ware aard van dit beestje leren kennen, en ik vind het dan ook jammer er dit jaar even niet bij te zijn. Assistentenweekend editie 4 is echter alweer in aantocht!

Mijn collega (ex) AIOs in Maastricht, ik ben niet veel in Maastricht geweest, maar ook bij jullie heb ik mij als vreemde eend in de bijt altijd welkom gevoeld, bij de Journal clubs, tijdens de koffiepauzes, congressen en workshops. De nacht in de karaokebar in Lissabon is er een die we allemaal niet snel zullen vergeten!

Thomas \& Eveline, Bas \& Lieke, Denise \& Frank, Liska, Koen \& Sharon, Janneke \& Sander, Jonas \& Anouk, Justin \& Lieke, Linda \& Jeroen, Ylaine \& Jeroen, Kostan \& Aleida, Sanne \& Maarten, Samira \& Wouter, in het laatste jaar van mijn promotietraject heb ik jullie pas leren kennen. Ik wil jullie bedanken voor de gezellige momenten in de weekenden dat jaar; waarin we al een aantal keren goed de bodem hebben geraakt.

Heerenjaarclub Numen, Arthur, Casper, Jack, Jeroen, Keyi, in 2004 kwamen wij allen tezamen, en dit jaar zullen wij 10 jaar bestaan. Van jullie heb ik inhoudelijk nooit iets geleerd dat aan dit proefschrift heeft bijgedragen, maar des te meer steun ervaren om dit project tot een goed einde te brengen. Dank voor de avonden, (cultuur)weekendjes, borrels, en de mooie herinneringen elk jaar aan het Festival van het Levenslied. De jaarclubdiners zijn door de jaren heen wat rustiger geworden, maar zeker niet minder gezellig. Ondanks onze diensten hoop ik dat deze nog steeds doorgezet worden, gevolgd door een volledig spontaan uitstapje naar de stad, enkel om te kijken hoe druk het is... 
Beste Math, menig middag heb ik toch ook bij jou aan tafel aan dit proefschrift zitten werken, en met veel plezier kan ik je nu dit boekje voorleggen. Bedankt voor je enthousiasme en interesse; vanaf de eerste dag heb ik me welkom gevoeld bij jou, en kon ik doen alsof ik thuis was. Ik betreur het dat ik Elly het boekje niet kan laten zien.

Pap en mam, zonder jullie was dit boekje er nooit gekomen. Vanaf het begin hebben jullie ervoor gezorgd dat wij een goede opleiding genoten, gingen studeren, en goed terecht zijn gekomen. Toen ik vertelde dat ik wilde promoveren, hadden jullie geen idee wat dat zou betekenen en waarom ik dat nou weer wilde doen, maar jullie hebben me door het hele traject onvoorwaardelijk gesteund. Als het even tegen zat, kon ik altijd even met jullie bellen en mijn frustraties kwijt, om vervolgens toch weer er bovenop te komen. Ook buiten deze promotie, of het nu is om 4 boortjes te breken op gewapend beton, of 6 jaar lang een schadevergoeding na te jagen, jullie staan altijd voor mij klaar. Dit boekje is voor jullie!

Martin, mijn paranimf, mijn beste vriend, mijn broer. Regel 1 is dat principes altijd tegen je gebruikt worden, en zo ook dit trikolon. Jij bent vanaf het begin mijn maatje geweest, en het lot wilde dat je het grootste deel van mijn promotietraject bij mij hebt gewoond. Zo'n beetje elke avond was jij het vaste aanspreekpunt voor de dagelijkse beslommeringen, en wisselde wetenschappelijke prietpraat zich af met wiskundige raadsels en wisselende probleemstellingen. Door de jaren heen heb ik van jou geleerd op te komen voor je eigen mening, ook als die 180 graden tegen die van de rest ingaat. Ik ben dan ook vereerd om je als paranimf achter mij te hebben staan. Vorig jaar zijn onze wegen gescheiden, maar ik zal die tijd nooit vergeten. Niets is sterker dan de band tussen broers.

Ten slotte, Harmke, jij bent pas tijdens dit onderzoeksproject in mijn leven gekomen, en hebt weinig van mij als fulltime onderzoeker meer gezien, maar des te meer van mij als AIOS die de laatste meters moest afleggen richting zijn promotie in weekenden en nachtdiensten. Jij hebt mijn leven in een ander perspectief geplaatst, en dit boekje zou een stuk minder waard zijn zonder jou. Ik ken niemand die mij meer op zijn gemak stelt, die samen van goed eten kan genieten, die wil danse tot 't mörgeleech, brak tekenfilms wil kijken op zondagmiddag, de sportschool ook nooit kan vinden, en die als vastelasten adviseur de anion gap en JAK2 mutatie kent. Ik hou van je tot aan de maan en terug. 
Curriculum vitae 


\section{Curriculum vitae}

Nick Wlazlo was born on May $30^{\text {th }} 1986$ in Sittard, the Netherlands. During childhood, he lived in Eijsden. In 2004, he graduated from secondary school (Sint Maartenscollege, Maastricht) and started his medical training at Maastricht University, Maastricht, the Netherlands. During the master phase of his study, he received the Top 3\% award twice. In July 2010, he obtained his medical degree cum laude. From September 2010 he started this AGIKO project, which was a collaboration project between the Catharina Hospital Eindhoven and the Maastricht University Medical Centre. As part of his PhD program, he also followed the research master in Epidemiology at the VU University (Amsterdam, the Netherlands) from January 2011 until December 2012, and graduated cum laude. Since October 2012, Nick is also working as a resident Internal Medicine at the Catharina Hospital Eindhoven. 
Scientific output 


\section{Scientific output}

\section{Articles}

1. Timmermans SH, Wlazlo N, Mom EM, Stoffers HE: [Thrombophlebitis of the leg: diagnosis and treatment by the general practitioner]. Ned Tijdschr Geneeskd 2010;154:81-85; A1098

2. Wlazlo N, Beijers HJ, Schoon EJ, Sauerwein HP, Stehouwer CD, Bravenboer B: High prevalence of diabetes mellitus in patients with liver cirrhosis. Diabet Med 2010;27:1308-1311

3. Wlazlo N, Bravenboer B, Pijpers R, de Rijk MC: [Low back pain and MRI-abnormalities: atypical polymyalgia rheumatica]. Ned Tijdschr Geneeskd 2011;155:32-36; A2300

4. Wlazlo N, Sauerwein HP, Schoon EJ, Stehouwer CD, Bravenboer B: [Diabetes mellitus and cirrhosis of the liver: a prognostically unfavourable combination]. Ned Tijdschr Geneeskd 2011;155:212-219; A2213

5. Wlazlo N, Winkens B, Van der Vleuten CPM, Peters WG: Near-peer teaching in het coschap interne geneeskunde: een gerandomizeerde cross-over trial. Tijdschr Med Onderwijs 2011;30:2-10

6. Wlazlo N, Greevenbroek MM: Lipid metabolism: a role for iron? Curr Opin Lipidol 2012;23:258-259

7. Wlazlo N, Peters W, Bravenboer B: Hypogonadism in a patient with mild hereditary haemochromatosis. Neth J Med 2012;70:318-320

8. Wlazlo N, van Greevenbroek MM, Ferreira I, Bravenboer B, Stehouwer CD: The diagnosis of non-alcoholic fatty liver disease. Aliment Pharmacol Ther 2012;35:204205

9. Wlazlo N, van Greevenbroek MM, Ferreira I, Jansen EJ, Feskens EJ, van der Kallen CJ, Schalkwijk CG, Bravenboer B, Stehouwer CD: Low-grade inflammation and insulin resistance independently explain substantial parts of the association between body fat and serum C3: The CODAM study. Metabolism 2012;61:17871796

10. Wlazlo N, van Greevenbroek MM, Curvers J, Schoon EJ, Friederich P, Twisk JW, Bravenboer B, Stehouwer CD: Diabetes mellitus at the time of diagnosis of cirrhosis is associated with higher incidence of spontaneous bacterial peritonitis, but not with increased mortality. Clinical science 2013;125:341-348

11. Wlazlo N, van Greevenbroek MM, Ferreira I, Jansen EH, Feskens EJ, van der Kallen CJ, Schalkwijk CG, Bravenboer B, Stehouwer CD: Iron metabolism is associated with adipocyte insulin resistance and plasma adiponectin: the Cohort on Diabetes and Atherosclerosis Maastricht (CODAM) study. Diabetes Care 2013;36:309-315 
12. Wlazlo N, van Greevenbroek MM, Ferreira I, Jansen EH, Feskens EJ, van der Kallen CJ, Schalkwijk CG, Bravenboer B, Stehouwer CD: Activated complement factor 3 is associated with liver fat and liver enzymes: the CODAM study. Eur J Clin Invest 2013;43:679-688

\section{Oral presentations}

Internistendagen, NIV, Maastricht, the Netherlands, April 2010

Internistendagen, NIV, Maastricht, the Netherlands, April 2011

ADDDG meeting, ADDDG, Perthshire, Scotland, May 2011

WEON 2011, Netherlands Epidemiology Society, Amsterdam, the Netherlands, June 2011

Najaarscongres NvGE, NvGE, Veldhoven, the Netherlands, October 2011

Regionale endocrinologie bijeenkomst ZO-Nederland, Nijmegen, the Netherlands, Nov 2011

Junior NVDO meeting, NVDO, Soesterberg, the Netherlands, May 2012

WEON 2012, Netherlands Epidemiology Society, Amsterdam, the Netherlands, June 2012

NVDO meeting, NVDO, Oosterbeek, the Netherlands, Nov 2012

NVDO meeting, NVDO, Oosterbeek, the Netherlands, Nov 2013

\section{Poster presentations}

EASD 46, European Association for the Study of Diabetes, Stockholm, September 2010

EASD 47, European Association for the Study of Diabetes, Lisbon, September 2011

The International Liver Congress 47, European Association for the Study of the Liver, Barcelona 2012 

